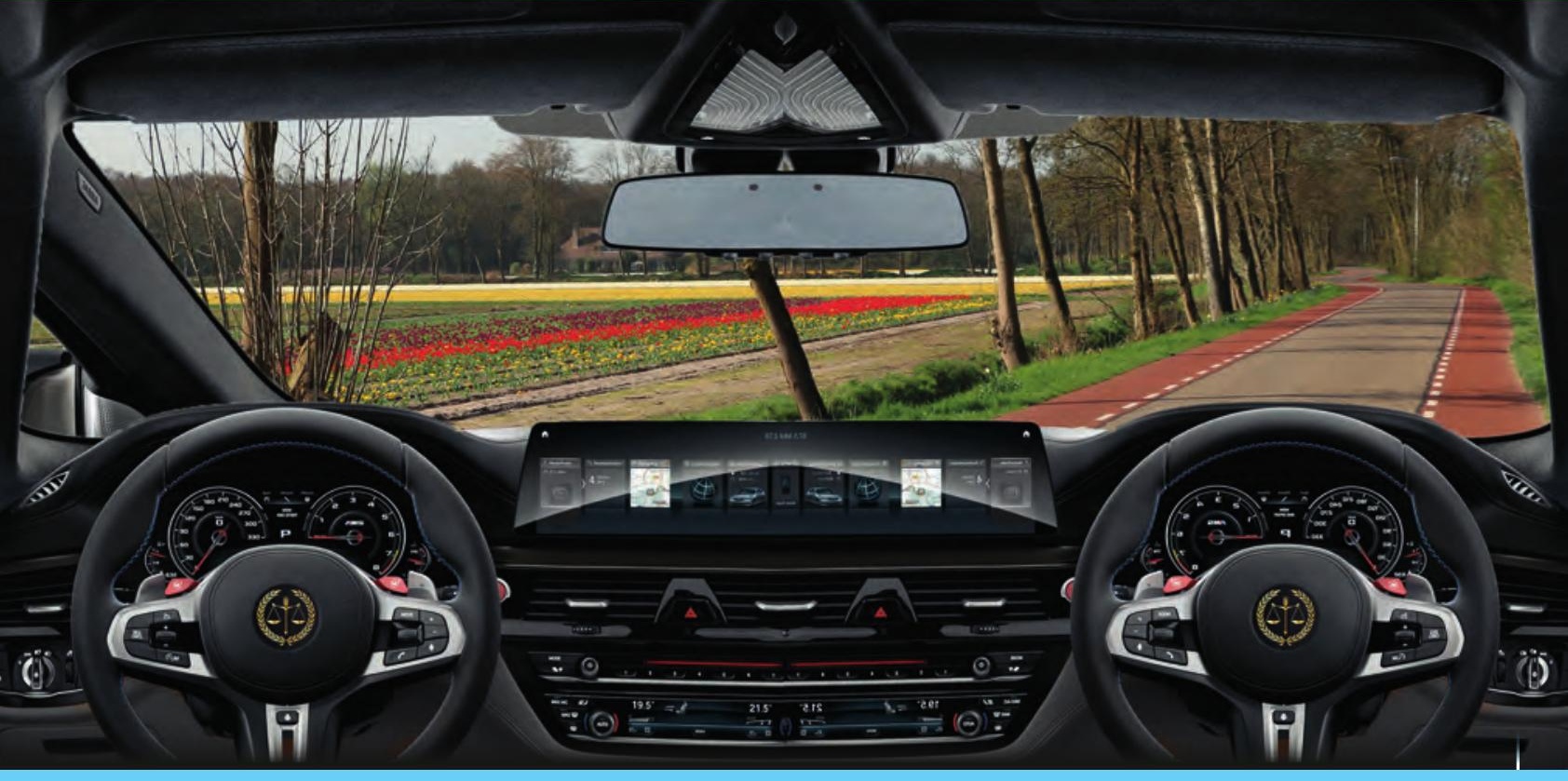

Hard and soft

IT governance maturity

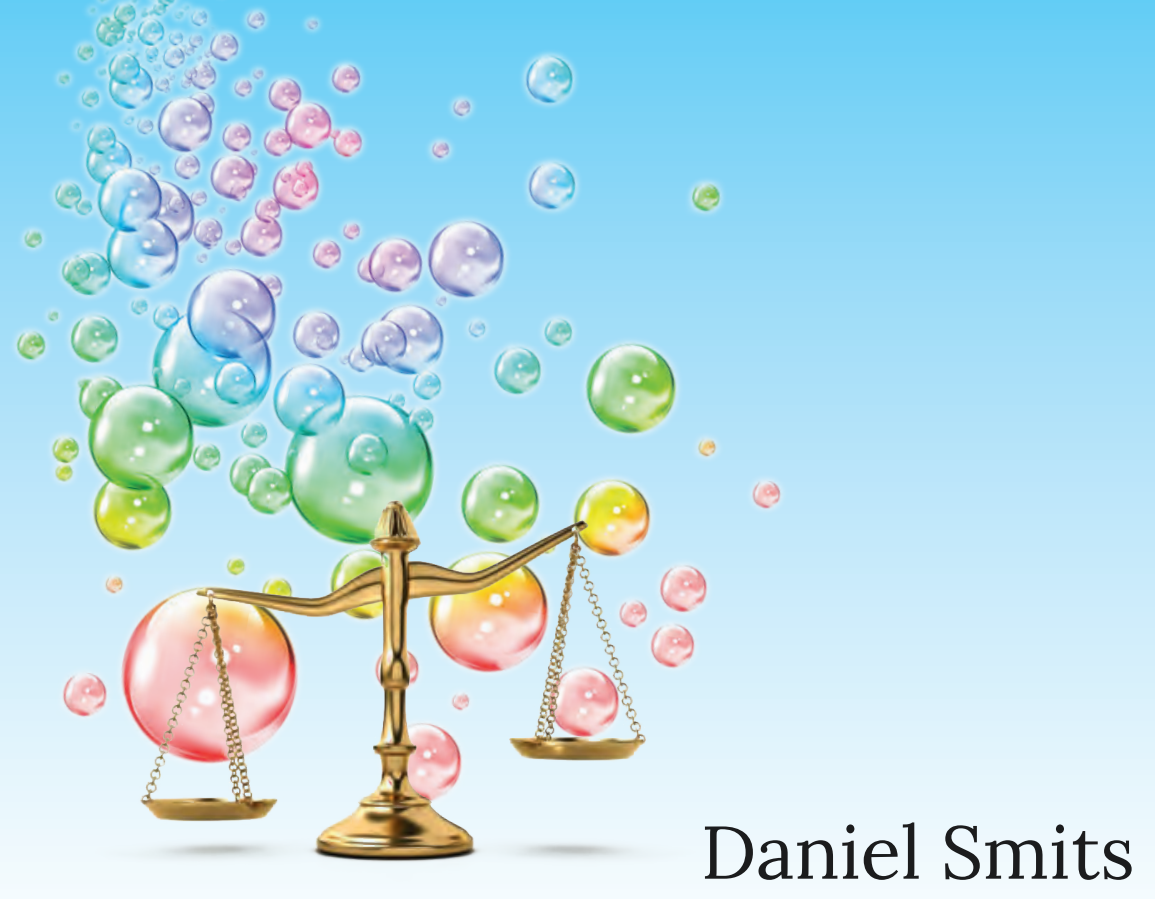




\section{HARD AND SOFT I T GOVERNANCE MATURITY}

Daniel Smits 



\title{
HARD AND SOFT IT GOVERNANCE MATURITY
}

\section{I S S E R TATION}

\author{
to obtain
}

the degree of doctor at the University of Twente, on the authority of the rector magnificus

$$
\text { Prof. Dr. T.T.M. Palstra, }
$$

on account of the decision of the Doctorate Board,

to be publicly defended

on Wednesday the $23^{\text {rd }}$ of January 2019 at $12: 45$ hours

by

Daniel Smits

Born on the $25 \mathrm{th}$ of December 1963

in Basel, Switzerland 
This dissertation has been approved by:

\section{Supervisor:}

\section{Prof. Dr. J. van Hillegersberg}

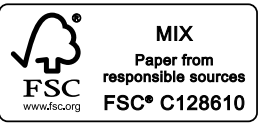

Printed by: IPSKAMP

Lay-out: Sanne Kassenberg, persoonlijkproefschrift.nl

ISBN: 978-90-365-4718-5

DOI: $10.3990 / 1.9789036547185$

(C) 2018 Daniel Smits, The Netherlands. All rights reserved.

No parts of this thesis may be reproduced, stored in a retrieval system or transmitted in any form or by any means without permission of the author.

Alle rechten voorbehouden. Niets uit deze uitgave mag worden vermenigvuldigd, in enige vorm of op enige wijze, zonder voorafgaande schriftelijke toestemming van de auteur. 


\section{Graduation committee}

Chairman/secretary Prof. Dr. T.A.J. Toonen, University of Twente

Supervisor

Prof. Dr. J van Hillegersberg, University of Twente

Members

Prof. Dr. M.E. Iacob, University of Twente

Prof. Dr. T. Bondarouk, University of Twente

Prof. Dr. W. Van Grembergen, University of Antwerp

Prof. Dr. E.W. Berghout, University of Groningen

Prof. Dr. Dr. h.c. Dr. h.c. J. Becker, WWU Münster

Prof. Dr. ir. L.J.M. Nieuwenhuis, University of Twente

Prof. Dr. S. Brinkkemper, Utrecht University 


\section{Preface}

When I started my career I worked as a programmer in languages including assembler and C. However, several decades later I shifted to the front side: the contact between business and IT. One is soon confronted with the question of how to design the IT governance (ITG) of an organisation. Important topics include architecture and portfolio management and these form some of the starting points of this investigation. In practice the ways in which organisations handle these vary considerably. After several years I concluded that no better solution for ITG practice exists.

The rationale behind this research was the absence of usable frameworks and models to implement ITG in practice.

Based on countless meetings and discussions and previous publications such as Focus op IT bestuur: essentiële elementen van IT governance (Smits et al., 2008), I concluded that we were paying insufficient attention to the human side of ITG. This appeared to be a widespread phenomenon in assessments of existing models and frameworks. Given that I was unable to find a useable best-practice standard, framework or model in the literature, it occurred to me to design such a model. Integrating the human or social side into a model consisting of structures and processes is not easy. To make it usable in practice requires considerable care. This issue inspired the idea for my research.

The aforementioned book's cover features a photograph of a dashboard with two steering wheels. This was used as a metaphor for the dilemma that an organisation is governed in several places or levels and in different ways.

This research is first and foremost concerned with the combination of hard and soft ITG factors, elaborated into 12 focus areas. The metaphor above still applies but the question might be how many steering wheels should be drawn. The colours on the cover took inspiration from Caluwé and Vermaak's (2004) colour model. A scale has been added to visualise the challenge of balancing all of these factors.

The starting point of this research was the expression "The strength of a chain is that of its weakest link". One of the first steps was to determine with which links is ITG concerned. The answers appeared to be different from what was originally expected. Furthermore, it was positive to realise that it was possible to demonstrate that these 12 links are not only relevant for ITG, but for corporate governance too. Thus, the complete organisation, not only IT.

This thesis describes the results of three cycles of the design process of an ITG model and the accompanying assessment instrument for practice. The result of this design process is 
an instrument that in its current form can be used in practice. However, some improvements would be desirable to render it easier to use in practice. In the case of corporate governance, it remains to be seen whether links (focus areas) are required as supplements.

Therefore, there are numerous potential topics for future research, and accordingly my research continues. In 2018, students conducted case studies with version 4 of the instrument. Next year more case studies will follow using version 5.

The ultimate goal is to create an instrument that can be used by members of an organisation without interviews to correct the results and make the instrument available to the public. As such, the instrument not only indicates the current level of maturity of an organisation, but also the actions required to improve and ultimately reach the desired level.

Daniel Smits, November 2018 


\title{
CONTENTS
}

\author{
PREFACE \\ CONTENTS
}

PART I: INTRODUCTION AND MOTIVATION FOR THE R E S E A R C H

1. INTRODUCTION TO THE RESEARCH 15

$\begin{array}{ll}\text { 1.1. The discipline of IT governance } & 16\end{array}$

\begin{tabular}{ll} 
1.2. & Research motivation \\
\hline
\end{tabular}

1.3. Research area 21

$\begin{array}{lll}\text { 1.4. Scope of the research } & 29\end{array}$

1.5. Summary of this section 30

2. RESEARCH QUESTIONS AND METHOD 31

2.1. Research goal 31

2.2. Research method 34

2.3. Research model $\quad 43$

$\begin{array}{ll}\text { 2.4. Thesis structure } & 47\end{array}$

2.5. Summary of this section $\quad 53$

PART II: COLLECT PRIOR KNOWLEDGE AND ASSESS

PRACTICAL RELEVANCE

3. COLLECT PRIOR RESEARCH KNOWLEDGE AND ASSESS PRACTICAL RELEVANCE 57

3.1. Introduction 57

3.2. Research questions $\quad 59$

$\begin{array}{ll}\text { 3.3. Research method } & 59\end{array}$

3.4. Results of the study 62

$\begin{array}{ll}\text { 3.5. Discussion } & 72\end{array}$

$\begin{array}{ll}\text { 3.6. Conclusions } & 75\end{array}$

$\begin{array}{lll}\text { 3.7. Summary of this section } & 77\end{array}$ 
4. SYSTEMATIC LITERATURE REVIEW ON ITG (2018 UPDATE)

4.1. Introduction $\quad 79$

4.2. Systematic literate study on ITG: 2018 update 80

4.3. Discussion 83

4.4. Conclusion 88

4.5. Summary of this section $\quad 89$

PART III: DESIGN AND EVALUATION OF THE MIG MODEL AND THE MIG ASSESSMENT INSTRUMENT 91

5. THE DESIGN OF THE MIG MODEL 93

5.1. Introduction 93

5.2. Research method 94

5.3. The design of the initial model 97

5.4. The design of the MIG model 100

5.5. The design of the maturity levels in the MIG model 107

5.6. Completing the MIG model 121

$\begin{array}{lll}\text { 5.7. Conclusions } & 129\end{array}$

5.8. Summary of this section 131

6. THE DESIGN AND EVALUATION OF THE MIG ASSESSMENT INSTRUMENT 133

6.1. Introduction 133

6.2. Research method 134

6.3. The first cycle: MIG assessment v. 1(2015) 137

6.4. The second cycle: MIG assessment v. 2 (2016) 142

6.5. The third cycle: MIG assessment v. 3 (2017) 147

$\begin{array}{lll}\text { 6.6. Conclusion } & 147\end{array}$

$\begin{array}{lll}\text { 6.7. Summary of this section } & 148\end{array}$ 
PART IV: USE, IMPROVEMENT AND VALIDATION OF THE MIG INSTRUMENT

7. RESULTS FROM THREE CYCLES OF CASE STUDIES 151

$\begin{array}{ll}\text { 7.1. Introduction } & 151\end{array}$

7.2. Research method 152

7.3. The first cycle: case studies in $2015 \quad 153$

7.4. The second cycle: case studies in $2016 \quad 160$

7.5. The third cycle: case studies in $2017 \quad 164$

$\begin{array}{lll}\text { 7.6. Summary of this section } & 178\end{array}$

8. THE LINK BETWEEN CORPORATE GOVERNANCE AND IT GOVERNANCE 181

8.1. Introduction 181

8.2. Research method 183

8.3. Results of the systematic literature review 184

$\begin{array}{lll}\text { 8.4. Discussion } & 187\end{array}$

$\begin{array}{ll}\text { 8.5. Conclusion } & 190\end{array}$

8.6. Summary of this section 191

PART V: CONCLUSIONS, LIMITATIONS AND FUTURE RESEARCH 193

9. CONCLUSIONS AND FUTURE RESEARCH 195

$\begin{array}{lll}\text { 9.1. Introduction } & 195\end{array}$

$\begin{array}{ll}\text { 9.2. Conclusions } & 196\end{array}$

$\begin{array}{ll}\text { 9.3. Contribution } 205 & 205\end{array}$

9.4. Limitations 205

$\begin{array}{lll}\text { 9.5. Future research } & 206\end{array}$

ACKNOWLEDGEMENTS 209

R E F E R E N C E S 211

PUBLICATION LIS T 
APPENDIX C THE MIG ASSESSMENT INSTRUMENT V. 3

APPENDIX E HANDOUT INTERVIEW MIG ASSESSMENT V. 3

APPENDIX F RESULTS CASE STUDIES BETWEEN 2015 A ND 2017 


\section{Part I}

\section{Introduction and motivation for the research}

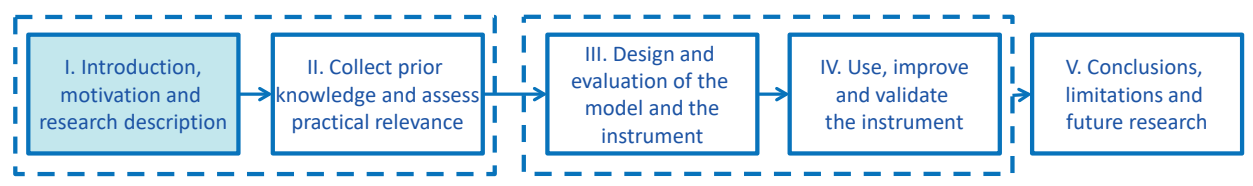

Figure 1 Thesis Part I. Introduction, motivation and research description

\section{Section}

1. Introduction and motivation for the research

2. Research questions and method 


\section{Section 1}

\section{Introduction to the research}

The goal of the research presented in this thesis is to determine how the IT governance (ITG) of an organisation can grow in maturity to become more effective.

ITG is a relatively new topic (Van Grembergen, 2004), with the first publications appearing in the late 1990s. Although a considerable body of literature on ITG exists, definitions of ITG in the literature vary considerably (Webb et al., 2006, Lee and Lee, 2009).

The initial rationale behind this research was the experience that ITG represents an ongoing concern for organisations worldwide. There simply does not seem to be a common body of ITG knowledge or a widely used ITG framework. In practice, organisations use all kinds of frameworks or methods for ITG. This research thus aims to add to the knowledge of how to implement and improve ITG.

A study by Weill and Ross (2004) highlighted that firms with superior ITG enjoy more than $20 \%$ higher profits than firms with poor governance. How then can organisations achieve this goal of superior ITG?

We define superior ITG as more effective ITG (relative to industry averages) and assume that ameliorating ITG maturity results in improved ITG. This research is intended to design an ITG maturity model that helps organisations to improve their ITG.

In the literature, constructs such as dimensions, focus areas or principles are often used to refine the concept of ITG. Dimensions may include IT compliance management or business/ IT alignment (Novotny et al., 2012); examples of focus areas are value delivery or resource management (ITGI, 2003b); and principles could be strategy or responsibility (ISO/IEC 38500, 2008). For applicability in practice we use the more practical term 'discipline'. Examples of disciplines comprise architecture or portfolio management, which directly relate to roles or functions in an organisation, such as architect or portfolio manager. 


\subsection{The discipline of IT governance}

Scholars define ITG in diverse ways. Brown and Grant classify previous literature regarding ITG into two separate historical streams "that follow parallel paths of advancement" (2005):

1. IT Governance Forms

In this stream, ITG is associated with underlying decision-making structures, including attempts to define the various structural forms that governance models might adopt.

\section{IT Governance Contingency Analysis}

"In this stream, research focuses on the "why and how" of IT governance fit. Rather than investigate basic structural options, researchers attempt to understand which option is best for which organization, through an analysis of factors that affect individual IT governance framework success“ (Brown and Grant, 2005).

The number of ITG publications began to grow from 2006/2007 (Smits and van Hillegersberg, 2014a). In this thesis we use three streams of ITG as a starting point: IT auditing, decisionmaking and ITG as an integral part of corporate governance (Musson, 2009).

In a systematic literature review of contemporary research, we found six streams based on two perspectives. We use three streams of ITG as a starting point: IT auditing, decision-making and ITG as an integral part of corporate governance (Musson, 2009). The first perspective handles the scope of ITG; the second handles the direction in which it works.

The streams are summarised in Table 1. More details can be found in Section 3.

\begin{tabular}{|l|l|}
\hline \multirow{3}{*}{ View } & IT governance stream \\
\hline \multirow{5}{*}{ Direction } & 1. IT audit \\
\cline { 2 - 2 } & 2. Decision-making \\
\cline { 2 - 2 } & 3. Part of corporate governance, conformance perspective \\
\cline { 2 - 2 } & 4. Part of corporate governance, performance perspective \\
\cline { 2 - 2 } & B. Totown-down \\
\hline
\end{tabular}

Table 1 Six ITG streams

The stakeholders involved in the discipline of IT governance are different for each stream. 


\section{Scope of ITG}

Different streams can be distinguished, with some using a small scope and others a broad scope.

Stream 1: A clear proponent of the broad definition and founder of COBIT is the IT Governance Institute. COBIT is the well-known framework formerly known as Control Objectives for Information and Related Technology. In 1996 ISACA released the first edition of the COBIT framework. This was originally released as a set of control objectives to support financial auditors and IT auditors in practice. It was expanded in subsequent years with control guidelines (version 2), management guidelines (version 3) and ITG guidelines in 2005 (version 4.0) and 2007 (version 4.1).

In 2011, ITIL (including ISO 20000), the international standard for IT service management, remained the most frequently mentioned external framework used as a basis for ITG (ITGI). Some researchers define ITG "as the process by which decisions are made around IT investments" and claim ITIL version 3, released in 2007, can provide a well matured framework for ITG (Nabiollahi and Sahibuddin, 2008).

Stream 2: Weill and Ross (2004) use define ITG simply as "the decision rights and accountability framework for encouraging desirable behaviour in the use of IT". These authors can be deemed the main contributors to the stream focusing on decision-making, and consider ITG from a decision-making perspective. As components of ITG, this stream uses elements such as IT decisions or decision archetypes (Weill and Ross, 2004, Weill and Ross, 2005). Others complement this with the context in which the decision is made (Xue et al., 2008).

Stream 3 and 4: At one end of the continuum, emphasis is placed on corporate conformance, and at the other end, a concern with corporate performance (Bhimani and Soonawalla, 2005). Given that our research focuses on performance rather than conformance we must differentiate between both aspects of corporate governance. We define 'corporate governance, conformance perspective' as being related to rules and regulations, and 'corporate governance, performance perspective' as pertaining to performance and value creation.

\section{Working direction of ITG}

Whereas the top-down view is related to structure, processes and planning, the bottom-up view is related to social aspects like culture, behaviour and collaboration. To explain this view we make a side-step to institutional economics, in which two contrasting worldviews coexist. 
They have their origins in the $18^{\text {th }}$ century Enlightenment and can be described as top-down or bottom-up (Easterly, 2008), a concept that can also be found in the corporate governance literature (Landier et al., 2012, Clark and Wójcik, 2003, Earl and Potts, 2011).

Stream 5: Most scholars perceive governance as a top-down phenomenon, often based on structure, processes and planning. Structure represented one of the historical perspectives regarding ITG. Brown and Grant's (2005) "IT Governance Forms" historical stream is closely related to IT organisational structures, while their second stream "ITG continency analysis" can be connected to decision-making. Research on the individual and multiple contingencies affect IT organisational structure decisions. Both can be seen as appearances from the top-down view.

The top-down view of ITG sees the governance of an organisation as being determined by the rules written by the management and leaders of the organisation.

Stream 6: Another view on ITG is bottom-up. The bottom-up view sees ITG as emerging spontaneously from the social norms, customs, traditions, beliefs and values of employees within the organisation, in which the governance simply formalises what has already been shaped in large part by the attitudes of individuals.

Followers of stream 6 often criticise structural and top-down planning processes. For example, Lindblom (1959) proposes an alternative to the analytical planning approach by "muddling through" with the argument that real world problems are far too complex to solve this way. The complex process of IT or ITG cannot be understood, let alone managed, by approaches consisting of control processes, planning or information systems alone (Ciborra et al., 2000). The definition of IT as a hybrid of technical systems and human users spells out the composite nature of our field (Ciborra, 1998). Ciborra deconstructed managers' and consultants' too obvious assumptions such as alignment, planning or management command and control systems (Maes and Huizing, 2005). Schwarz and Hirschheim (2003) suggest that IT executives should approach their governance structure as an "architecture" instead of formalised hierarchies, and argue that researchers need to change their view from IT as constituting a structure to "embrace a more social and dynamic existence". Dietz and Hoogervorst (2012) argue that a paradigm shift is required to deal with the challenges faced by modern enterprises. The authors identify three generic objectives: employee empowerment, because current employees are highly educated knowledge workers; competence-oriented governance, in order to master complexity; and the requirement for standardisation and integration, which can only be achieved by deliberate 
enterprise design. Competence can be seen as a replacement for capability (Williamson, 1999). In this thesis we prefer the use of the term 'capabilities'.

\subsection{Research motivation}

In 2008 we have written a book covering essential elements of ITG (Smits et al., 2008). Reviews of the book were positive and it was used by practitioners and universities of applied sciences for student education. However, some made the remark that the human element (the social side, human behaviour) was lacking. Others suggested this would be an interesting topic for PhD research.

In practice, frameworks are used to enable effective ITG. As this thesis will illustrate available frameworks from theory and practice do not match and do not cover the soft side of ITG. Governance is about people too. This intimates that human behaviour and social aspects are just as important. In practice there is a need for frameworks that cover the soft side too.

The most challenging part of this research is to combine the hard and the soft side of ITG. The model and instrument described in this thesis are a first step in building a theory to integrate both the hard and soft sides of IT governance.

The intention of the research in this thesis is to design a practical model and instrument using existing literature and design science.

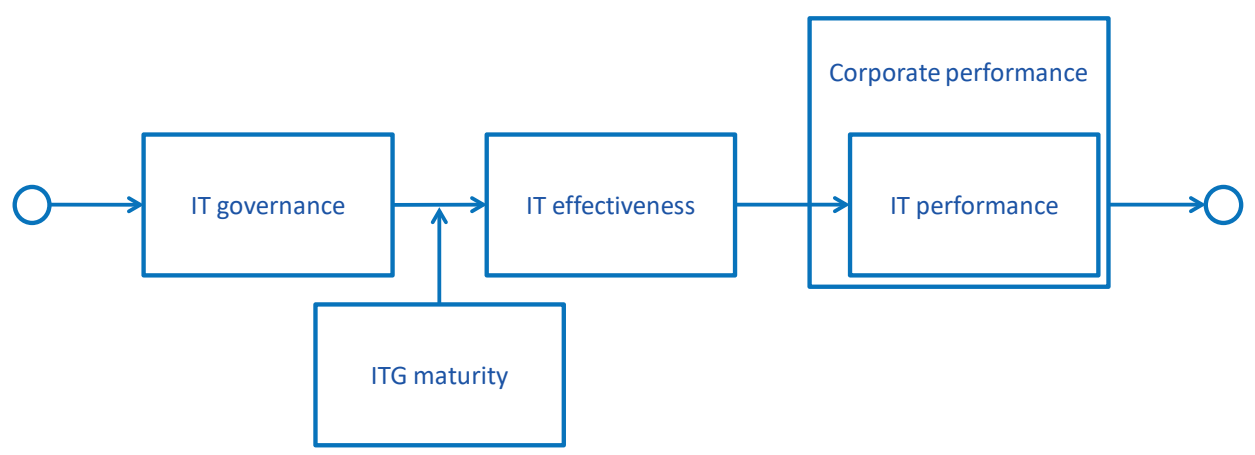

Figure 2 Relation between ITG and firm performance

The approach in this thesis is grounded on the assumption that: (Figure 2)

- $\quad$ Improving ITG maturity results in improved IT effectiveness;

- $\quad$ Improving IT effectiveness results in improved IT performance;

- $\quad$ Improved IT performance results in improved corporate performance. 
The research presented in this thesis has a multi-disciplinary nature. Its roots are in information systems research, but there are also strong links to organisational and social theory (see section 2.1). Organisations can be defined as social units of people that are structured and managed to pursue collective goals.

Corporate governance and IT governance (ITG) represent important and challenging topics for organisations. It is widely acknowledged that corporate governance and ITG are related. However, little is known regarding how this relationship actually works. Corporate governance is of "enormous practical importance" (Shleifer and Vishny, 1997).

The causal relationship of how ITG promotes firm performance remains unclear (Vejseli and Rossmann, 2017, Wu et al., 2015). Therefore, a better understanding of this relationship is required. However, for this thesis further investigation of this relationship is out of scope. The focus is on enhancing ITG maturity integrating hard and soft elements. For now, we postulate that ultimately, IT and corporate performance will benefit from higher ITG maturity.

This research - as stated before - is intended to design an ITG maturity model. The maturing entities in our ITG maturity model are "organisational capabilities". An ITG maturity model is a conceptual multi-stage model that describes typical patterns in the development of organisational capabilities in order to assess the as-is situation and to derive and prioritise improvement measures (Pöppelbuß et al., 2011).

Thus, we look at ITG from two perspectives:

- $\quad$ An organisational perspective referred to as "hard governance";

- $\quad$ A social perspective referred to as "soft governance".

Does ITG maturity have a significant positive impact on IT performance and firm performance? Some studies suggest a significant positive impact (Liang et al., 2011, Simonsson et al., 2010, Dodds, 2004, Weill and Ross, 2004), whereas others have failed to find a clear positive correlation (Tugas, 2010, Tanriverdi, 2006), and still others argue that there may be a considerable time delay between an improvement in ITG maturity levels and perceived benefits (Yuwono and Vijaya, 2011).

This research is grounded in the assumption that in order to advance in maturity, organisations should pay attention to both the hard and soft sides of IT governance (ITG). The hard side is related to processes and structure; the soft side to social aspects like behaviour and organisational culture. 
We argue that immature or low levels of ITG maturity are the cause of a lack of effective ITG. In order to improve ITG, we opted to design a maturity model for hard and soft ITG, because to the best of our knowledge this does not exist even though it is required in practice. This maturity model is intended to help organisations in improving their hard (structure, processes) and soft (behaviour, collaboration) ITG.

\subsection{Research a rea}

In this section we will introduce four topics that are relevant to our research:

- $\quad$ IT governance;

- $\quad$ Corporate governance;

- $\quad$ Hard and soft governance;

- $\quad$ Maturity models.

The introduction of the topics is based on the results of the systematic ITG literature review described later in the thesis.

\subsubsection{T governance}

A survey conducted by the ITGI (2011) showed that in practice frameworks are the most important enablers for effective ITG. Other enablers include toolkits, benchmarking, certifications, networking, white papers and ITG-related research. Some of the frequently cited frameworks comprise COBIT, ITIL, ISO/IEC 27001, ISO/IEC 17799 and BS 7799 (Musson, 2009). The frameworks used for ITG vary considerably, as can be seen in several global surveys from the ITGI addressed to $749 \mathrm{CEO}$-/CIO-level executives in 23 countries, and summarised in Table 2 (ITGI, 2011, ITGI, 2008)1. Unfortunately, the most recent Global survey from 2016 does not include a question concerning the use of IT governance frameworks. ${ }^{2}$

To illustrate the diverse nature of these frameworks, we added the column 'Content'.

1 The numbers are from the survey in 2008. The survey in 2011 consisted of 703 online surveys and 130 surveys by telephone. In 2011 the respondents were based in 21 countries.

2 The Global survey of 2016 is not publicly available. In inquiring whether this version also includes information on the use of ITG frameworks as in the former surveys with Kristen Kessinger (senior manager media relations ISACA), the response "unfortunately that was not a question asked in this survey" was received on 18 June 2018. 
Section 1

\begin{tabular}{|c|c|c|c|c|}
\hline Framework & Content & 2011 & 2007 & 2005 \\
\hline ITIL or ISO/IEC 20000 & Service management & $28 \%$ & $24 \%$ & $13 \%$ \\
\hline $\begin{array}{l}\text { ISO/IEC } 17799, \text { ISO/IEC } 27000 \text { or other } \\
\text { security frameworks }\end{array}$ & Information security & $21 \%$ & $10 \%$ & $9 \%$ \\
\hline Internally developed frameworks & Unknown/differ & & $14 \%$ & $33 \%$ \\
\hline Six Sigma & Quality & $15 \%$ & $2 \%$ & $5 \%$ \\
\hline COBIT (ISACA) & IT governance & $13 \%$ & $14 \%$ & $9 \%$ \\
\hline PMI/PMBOK & Project management & $13 \%$ & $1 \%$ & $3 \%$ \\
\hline Risk IT (ISACA) & Risk management & $12 \%$ & & \\
\hline IT assurance framework (ISACA) & IT assurance & $10 \%$ & & \\
\hline CMM or CMMI & $\begin{array}{l}\text { Software development or process } \\
\text { improvement }\end{array}$ & $9 \%$ & $4 \%$ & $4 \%$ \\
\hline ISO/IEC 38500 & IT governance & $8 \%$ & & \\
\hline $\begin{array}{l}\text { BMIS (Business Model for Information } \\
\text { Security, ISACA) }\end{array}$ & Information security & $8 \%$ & & \\
\hline PRINCE2 & Project management & $6 \%$ & $2 \%$ & \\
\hline Val IT (ISACA) & Enterprise value (IT investments) & $5 \%$ & $0 \%$ & \\
\hline TOGAF & Enterprise architecture & $3 \%$ & $0 \%$ & \\
\hline COSO ERM & Enterprise risk management & $2 \%$ & $1 \%$ & $4 \%$ \\
\hline
\end{tabular}

Table 2 Use of ITG frameworks (ITGI, 2008, 2011)

With 13\% growth for Six Sigma, 12\% growth for PMI/PMBOK, $11 \%$ growth for security frameworks, $4 \%$ growth for ITIL, $3 \%$ growth for TOGAF (from 0), and a $1 \%$ decrease for COBIT in a period of four years, there is no clear leader. Furthermore, it is clear that more general frameworks like Six Sigma are fast growers, too.

The relationship with project and portfolio management frameworks like PMI/PMBOK and PRINCE2 as well as architecture frameworks like TOGAF can be illustrated with cases found in academic research in which ITG is implemented using portfolio management and architecture (Wittenburg et al., 2007).

COBIT uses a classification consisting of five focus areas: strategic alignment, value delivery, resource management, risk management and performance measurement. The latest COBIT release is COBIT 5.0 (ISACA, 2012). In COBIT 5.0, the concepts and ideas contained in these focus areas are maintained and built upon in the framework, but the focus areas themselves are no longer explicitly visible in the framework (Bernard, 2012, ISACA, 2012, Cobo et al., 2014).

A recent literature survey by Novotny et al. (2012) on the dimensions and operationalisation of ITG reveals nine ITG dimensions, which are listed in Table 3. 


\begin{tabular}{|l|l|}
\hline \multicolumn{3}{|l|}{ Dimension } \\
\hline \multirow{5}{*}{ Input } & IT compliance management \\
\cline { 2 - 2 } & IT risk management \\
\cline { 2 - 2 } & IT decision authority and responsibility \\
\cline { 2 - 3 } & IT performance and quality measurement \\
\cline { 2 - 3 } & IT investment management \\
\cline { 2 - 3 } & IT resource and capability management \\
\cline { 2 - 3 } & ITG improvement \\
\hline \multirow{2}{*}{ Output } & Business/IT alignment \\
\cline { 2 - 3 } & Business value delivery \\
\hline
\end{tabular}

Table 3 Dimensions of ITG (Novotny et al., 2012)

Four dimensions are clearly complementary to the focus areas of COBIT 5.0: compliance management, decision authority and responsibility, investment management and ITG improvement.

Another well-known classification comprises the three layers of Peterson, O'Callaghan and Ribbers (2000): structural integration, functional integration and social integration. In 2004 this became better known (and somewhat simplified) as the trichotomy of structure, processes and relational mechanisms (Van Grembergen, 2004).

This classification may be concise and practical, but as among others Willson and Pollard (2009) have shown, ITG is not limited to structure, processes and mechanisms; it also relies on complex relationships, between history and present operations. Furthermore, cultural and human aspects are some of the factors that had the greatest influence on the implementation of ITG by 50\% of the participants of a large global survey conducted by ITGI (ITGI, 2011). Moreover, human behaviour was also included in the ISO/IEC 38500, the international standard for ITG. The standard defines six principles for directors and top management: responsibility, strategy, acquisition, performance, conformance and human behaviour (ISO/IEC 38500, 2008).

Various publications suggest that ITG constitutes an integral part of corporate governance (Van Grembergen et al., 2004, Lainhart and John, 2000, ITGI, 2003a). “The business dependency on IT means that the corporate governance issues cannot be solved without considering IT" (Van Grembergen et al., 2004). Little is known regarding the way in which this relationship works. This relationship can be described in several ways, such as from a performance (added value) or a conformance (rules and regulations) perspective. 


\subsubsection{Corporate governance}

Various definitions of corporate governance exist. Nerantzidis et al. (2012) analysed and summarised 22 definitions of corporate governance into a six-dimensional framework. This framework consists of institutional, shareholder, governance, control, performance and stakeholder dimensions.

Given that our research focuses on performance, we adopt the second-most used corporate governance definition from the Organization for Economic Co-operation and Development (OECD):

"corporate governance involves a set of relationships between a company's management, its board, its shareholders and other stakeholders. It also provides the structure through which the objectives of the company are set, and the means of attaining those objectives and monitoring performance are determined" (Nerantzidis et al., 2012).

There is considerable disagreement regarding good or bad governance mechanisms (Shleifer and Vishny, 1997). A dominant perspective in corporate governance studies is the agency theory (Dalton et al., 1998, Shleifer and Vishny, 1997), in which large corporations are reduced to two participants: managers and shareholders.

An alternative trend in corporate governance was strengthened following a series of large corporate scandals involving companies such as Enron, WorldCom and Tyco, highlighting the importance of stakeholders beyond shareholders (Kochan, 2003). A broader perspective of corporate governance was reintroduced, and corporations were reminded of their corporate and social responsibilities. A "multitheoretic approach" to corporate governance is required to determine the essential focus areas to improve organisational functioning (Daily et al., 2003).

This definition also represents an example of a broader perspective on corporate governance, as it covers the institutional, governance, shareholder, stakeholder and performance dimensions.

\subsubsection{Hard governance}

In this research, hard governance covers structural integration and functional integration:

- Structural integration: formal structural mechanisms with increasing complexity and capability, ranging from direct supervision, liaison roles, task forces and temporary teams to full-time integrating roles and cross-functional units and committees for IT (Peterson et al., 2000, Galbraith, 1974, Mintzberg, 1979). Informal structural integration comprises unplanned cooperative activities. Under complex and dynamic conditions, informal structural mechanisms support formal structural integration (Mintzberg, 1979). 
- $\quad$ Functional integration: the system of IT decision-making and communication processes (Luftman and Brier, 1999). The decision-making processes and decision-making arrangements (Sambamurthy and Zmud, 1999) are redefined in a later stage as "decision rights and accountability framework" (Weill and Ross, 2004). The communication processes describe the (in)formal communication and mutual adjustments among stakeholders (Galbraith, 1974, Mintzberg, 1979).

We define the hard side of ITG as the functional aspects of governance such as structures, processes and the formal side of decision-making. These aspects are also defined as elements of organisational design. We define hard governance as the organisational aspects of governance, linking it to functional aspects like structure, process and the formal side of decision-making. Structural integration mechanisms for ITG describe formal integration structures and staffskill professionalisation.

\subsubsection{Soft governance}

People represent the most important assets of an organisation. People do not work or think in terms of process and structure only; human behaviour and organisational culture are equally important aspects of governance. A survey by the IT Governance Institute showed that the culture of an organisation was deemed by $50 \%$ of the participants as one of the factors that most influenced the implementation of ITG, surpassed only by "business objectives or strategy", which scored 57\% (ITGI, 2011).

Thus, governance is about people too, which intimates that human behaviour and social aspects are just as important. Soft governance requires greater attention (Mettler and Rohner, 2009, Rogers, 2009, ITGI, 2011, Davies, 2012). Improvements are needed less in terms of structure and process and more in terms of the human or social aspects of governance (Davies, 2012). To be able to grow in maturity, organisations should pay attention to the hard and soft aspects of governance (ITGI, 2011).

The distinction between hard and soft governance is becoming more common in ITG research (Tucker, 2003, Moos, 2009, Cook, 2010a, Uehara, 2010, Tarmidi et al., 2012, Smits and van Hillegersberg, 2014b).

The division of governance into hard and soft governance has been made in the past (Moos, 2009, Tucker, 2003, Cook, 2010b, Uehara, 2010, Tarmidi et al., 2012). For instance, Moos (2009) differentiates between legislation and "softer" forms of governance based on persuasion and advice or obligation, precision and delegation (Tucker, 2003). Related to participatory 
governance, Cook (2010a) writes that "rules and structures" are "far less effective" than soft governance.

\section{Sof t power}

Uehara (2010) and Tarmidi et al. (2012) separate hard and soft ITG using the soft power theory. Joseph Nye (1990) founded the soft power theory, pertaining to "intangible power resources such as culture, ideology, and institutions". The basic concept of power is the ability to influence others to get them to do what you want. According to Nye (2004), this can be achieved in one of three major ways: threaten them with sticks; pay them with carrots; or attract them or co-opt them, so that they want what you want. If you can attract others to want what you want, it costs you much less in carrots and sticks.

Nye's research attended to world politics, but the same is true on a much smaller scale. Parents of teenagers know that if they shape their children's beliefs and preferences, they have greater and more enduring power than if they merely rely on active control. The same is true for members of an organisation.

In "The Bases of Social Power", French et al (1959) describe six bases of power: rewarding (carrots), coercive (sticks), legitimate (functions or roles), referent (soft power), expert (knowledge and science) and informational (relevant information or argument). Referent power concerns the association between individuals or groups and is strongly related to "our" soft governance. The management and psychology literature has long promoted the benefits of using referent power (soft power) over coercive power (hard power), because the former has the broadest range and yields the greatest influence relative to other bases (Cristo, 2005).

Soft power has attracted criticism, too. Criticism particularly pertains to the use of soft power on a state level: "despite its popularity soft power remains power of confusion" (Fan, 2008). Policymaking at the state level is "far more complicated than at personal level" which "significantly reduces the effect of soft power" (Fan, 2008). This might be true at a state level, but this research is at the organisational level and focuses on individuals and groups, a less complicated situation than research at the state level.

Therefore, soft power is sufficient for our purpose and close to how we see soft governance.

\subsubsection{Maturity Models}

The maturity concept emerged out of quality management. The concept of maturity stages was introduced by Crosby in 1979 with his "quality management process maturity grid" (Crosby, 1979, Wendler, 2012). Maturity models essentially represent theories concerning how organisational 
capabilities evolve in a stage-by-stage manner along an anticipated, desired or logical maturation path (Pöppelbuß and Röglinger, 2011). The concept of organisational capabilities is based on the resource-based-view used in the strategic management literature (Wernerfelt, 1984, Ulrich and Smallwood, 2004).

An organisation's capability is "the ability of an organization to perform a coordinated set of tasks, utilizing organizational resources, for the purpose of achieving a particular end result" (Helfat and Peteraf, 2003). The maturing entities in this research are organisational capabilities.

Maturity models can be seen as artefacts to determine a company's status quo and as "deriving measures for improvement" (Becker et al., 2009). The most well-known maturity model in the IT sector is CMM, of which version 1.0 was published in 1991 (Paulk et al., 1991). CMM was developed by the Software Engineering Institute (SEI) at Carnegie Mellon University. Interest in maturity emerged from quality management (SEI, 2010). In the 1930s, Walter Shewhart (1931) began his work on process improvement with his principles of statistical quality control. These principles were refined more than 50 years later by Deming, Crosby and Juran (Deming, 1986, Crosby, 1979, Juran, 1988).

The answer to the question "What makes organisational capabilities mature?" depends on which rationale is embraced, and tends to focus on the leverage points used in organisational change initiatives (Maier et al., 2012).

Maier (2012) discerns four leverage points that have been used in maturity models:

1. $\quad$ Existence and adherence to a structured process;

2. Alteration of organisational structure;

3. Emphasis on people;

4. Emphasis on learning.

The first two are related to hard governance; the latter two to soft governance. The maturity model developed in this thesis is a hybrid of these leverage points. A more detailed explanation of maturity models is included in Section 2.2.4.

\subsubsection{Summary of definitions}

In this section we define some important topics that are relevant to this research: corporate governance, ITG, maturity model, hard governance and soft governance.

Corporate governance We endorse Daily's remark that a "multitheoretic approach" to corporate governance is needed (2003), and adopt the "institutional" 
IT governance

Maturity model

Hard governance

Soft governance view on corporate governance (Nerantzidis et al., 2012). This can be summarised in the definition of corporate governance by Keasey and Wright (1993): "The structures, process, cultures and systems that engender the successful operation of the organization".

There are multiple relationships between corporate governance and ITG. In Section 1.1 we described six streams of ITG. Two of them see ITG as part of corporate governance.

The definitions applied to ITG also exhibit a lack of a clear shared understanding of the term ITG (Webb et al., 2006). Given that we see ITG as an integral part of corporate governance, we use a definition directly linked to the definition of corporate governance. We define ITG as "The structures, process, cultures and systems that engender the successful operation of the IT of the (complete) organisation". Thus, ITG is not restricted to the IT organisation.

We adopt the definition of Becker et al. (2009) of the maturity model: "A maturity model consists of a sequence of maturity levels for a class of objects. It represents an anticipated, desired, or typical evolution path of these objects shaped as discrete stages. Typically these objects are organizations or processes".

We define hard governance as the formal organisational aspects of governance, thus linking it to structure, process and the formal side of decision-making. Research has revealed that "structure" and "process" are the basic elements of corporate governance's institutional dimension (Nerantzidis et al., 2012). Hard governance is focused on structure and processes and is relevant for ITG and corporate governance.

We define soft governance as the human, social or informal aspects of governance, hence linking it to aspects like human behaviour, collaboration, organisational culture and the informal organisation. Soft governance is relevant for ITG and corporate governance. 


\subsection{Scope of the research}

We follow the stream in which ITG is seen as an integral aspect of corporate governance, and we are interested in the performance perspective. Organisational performance is determined by a large number of elements. Attempting to determine a direct relationship between ITG and organisational performance is not the focus of thesis.

Our approach is grounded in the following assumptions:

a. The proposition is that improving "ITG maturity" results in improving ITG;

b. Given that ITG is an integral part of corporate governance, the assumption is that improving ITG results in improving corporate governance;

c. Improving corporate governance results in improving organisational performance.

Numerous academic papers on the impact of corporate governance on organisational performance (thus concerning c.) exist. Including this third assumption into this thesis would add another highly voluminous research domain. In order to ensure this research's achievability, part c. falls beyond the scope of this thesis.

This places two parts within the scope of the thesis:

MRQ.I: How can we improve the ITG maturity of an organisation?

MRQ.II: Can we confirm the relevance of our findings for corporate governance?

Seeing ITG as an integral part of corporate governance also means that we expect (and demonstrate in Section 8) that the focus areas that are relevant for ITG are also relevant for corporate governance.

When ITG or corporate governance go awry, "the results can be devastating" (Wu et al., 2015). The bankruptcy of Enron in 2001 and other scandals at Tyco, Global Crossing, WorldCom and Xerox resulting in the enactment in the United States of the Sarbanes-Oxley Act are just a few examples. Employees, customers, suppliers and local societies suffered severe losses owing to managers driven by the possibilities of creating personal wealth through dramatic increases in the market prices of their shares (Kochan, 2003).

The impacts of IT governance on firm performance have been well-established in previous studies, yet there remains a gap explaining exactly how IT governance influences firm performance (Wu et al., 2015). ITG is positively related to business performance through IT and business process relatedness (Tanriverdi, 2006, Lazic et al., 2011). Weill and Ross (2004) 
present another excellent example of the linkage between ITG and corporate governance with corporate and IT decision-making. A third example comprises the relationship between corporate governance and ITG of Borth and Bradley (2009), in which ITG is presented as one of the key assets to govern.

\subsection{Summary of this section}

In this section, the discipline of ITG and the rationale for this research were introduced. Four key concepts were explained: ITG, corporate governance, hard governance (structure, process) and soft governance (behaviour, collaboration). We define corporate governance as the structures, process, cultures and systems that engender the successful operation of the organisation. Given that we perceive ITG as an integral part of corporate governance, we use the same definition with only an additional focus on IT.

The main research question is:

1. How can we improve the ITG maturity of an organisation?

2. Can we confirm the relevance of our findings for corporate governance?

Given that we could not find a maturity model for ITG that covers hard and soft governance, even though there is a need for such a model in research and practice, we designed an appropriate model in this research.

In the next section we describe the research goal, the research method, the research model and the structure of this thesis in greater detail. 


\section{Section 2}

\section{Research questions and method}

This section contains a description of the main research goal, the research method, the research questions and an outline of the thesis.

\subsection{Research goal}

The goal of the research described in this thesis is to determine how the ITG of an organisation can grow in maturity to become more effective. The research will deliver answers to questions regarding the implementation of the ITG. The main question of the research is:

[MRQ] How can the ITG of an organisation grow in maturity to become more effective?

\section{Practical relevance}

Our assumption is that a more effective ITG will be reached if the ITG of an organisation is more mature. Consultants in practice must base their approach on available frameworks and experience. Scientific support based on a reliable maturity model would be very helpful.

IT governance (ITG) is a "top 10" issue for CIOs. "IT governance continues to appear as an ongoing 'top 10' CIO management issue in Gartner's annual EXP survey of CIOs" was the conclusion of Gartner analyst J. Mahoney (2012), based on the Executive Programs' worldwide survey of more than 2,300 CIOs. In research described in this thesis (Smits and van Hillegersberg, 2013), some CIOs mentioned the importance of a "Maturity benchmark" for ITG (see Section 3.5 Results of the Delphi study), which requires the availability of a reliable assessment instrument.

In practice, frameworks are the most important enablers of effective ITG (ITGI, 2011). The list of frameworks frequently used for ITG is very diverse (ITGI, 2011, ITGI, 2008, Musson, 2009), but with the exception of COBIT and ISO/IEC 38500, these frameworks are not ITG-specific.

Currently (end-2018) the most important ITG framework for practice is COBIT. COBIT was released in 1996. The primary focus of COBIT is hard governance. New versions of COBIT display a gradual increase in attention to the soft side of ITG. In COBIT 5 a first holistic attempt was made to include the soft side. In COBIT 2019 (ISACA, 2018) the component "Culture, Ethics and Behaviour" was included as a management objective, adding a soft dimension to the process model of the COBIT framework. Thus, it seems the soft side of ITG receives more attention. However, human behaviour is not only process or structure related. 
In one of our studies we reported on the continuing mismatch between ITG theory and practice (Smits and van Hillegersberg, 2014a). The study describes the results of a literature review and a Delphi study with CIOs.

\section{Scientific relevance}

Information systems research is a discipline at the intersection of knowledge of the artificial (machines) and human behaviour (Gregor, 2002). Information Systems research is still very young "lacking the cumulative theory development" found in organisational and social research (Hevner et al., 2004, Gregor, 2006).

Early research on ITG included contingency studies from the organisation sciences (Sambamurthy and Zmud, 1999, Brown, 1997). Three primary modes of governance arrangements have emerged: the centralised, the decentralised and the federated governance mode (Sambamurthy and Zmud, 1999). IS researchers have found that the mode of corporate governance significantly influences the mode of ITG: organisations with centralised corporate governance tend to centralise their ITG, whereas organisations with decentralised corporate governance tend to decentralise their ITG (King, 1983, Leifer, 1988, Olson and Chervany, 1980). Other known contingency factors are the size of the organisation, the composition of the product portfolio (the ability to share resources across multiple products/services) and the level of IT-related knowledge possessed by the business managers.

More recently, researchers have investigated the effectiveness of structural governance mechanisms (Bowen et al., 2007, de Haes and van Grembergen, 2009, Huang et al., 2010, Herz et al., 2011, Prasad et al., 2012) or relational governance mechanisms (Ali and Green, 2012, Bradley et al., 2012) on ITG. Research confirms previous empirical findings that IS resources can contribute to organisational performance (Wu et al., 2015, Rai et al., 2006, Tanriverdi, 2006). Others see ITG and organisational performance connected by strategic alignment (Reich and Benbasat, 1996, Chan et al., 1997, Tallon and Pinsonneault, 2011, Wu et al., 2015), classifying strategic alignment against two dimensions: the intellectual (concentrating on planning) and the social (concentrating on the people involved). In spite of these efforts, the causal relationship between ITG and the performance of an organisation remains unclear (Vejseli and Rossmann, 2017), as well as the ITG mechanisms that affect organisational performance (Wu et al., 2015).

Demonstrating a direct causal relation between ITG and organisational performance is not the focus of this thesis. 
Our approach is grounded in the following assumptions: (see section 1.4)

a. The proposition is that improving "ITG maturity" results in improving ITG;

b. Given that ITG is an integral part of corporate governance, the assumption is that improving ITG results in improving corporate governance;

c. Improving corporate governance results in improving organisational performance.

Demonstrating a direct causal relation between corporate governance and organisational performance is considered equally unfeasible for this thesis. Thus part c. falls beyond the scope of this thesis.

In this thesis we focus on the design of an instrument to measure ITG maturity that can be used to improve ITG maturity (and thus ITG effectiveness) and a demonstration of the relevance of the results for corporate governance. "Designing useful artefacts is complex due to the need for creative advances in domain areas in which existing theory is often insufficient" (Hevner et al., 2004). To understand ITG, theory is required that "links the natural world, the social world and the artificial world of human constructions" (Gregor, 2006). Gregor $(2006,2002)$ distinguishes five interrelated classes of theory:

1. Analytic theory;

2. Theory for explaining;

3. Theory for prediction;

4. Theory for explaining and predicting;

5. Theory for design and action.

Some disqualify the first and second class of theory because of the omission of statements that can be empirically tested (Kerlinger, 1973, Doty and Glick, 1994). Gregor (2006) rates each type of theory as valuable. Many IS researchers fail to give a definition of their own view of theory (Gregor, 2006).

In this thesis we adopt Gregor's vision that each type of theory is valuable because we see them as interrelated. We need to design a maturity model (type 1) to be able to design an assessment instrument (type 1). The designed model and instrument can be used to learn and understand ITG by conducting case studies and uncover issues and explanations (type 2) to define testable propositions (type 3 and 4). Finally, the resulting theory (type 1 till 4) can be elaborated in our maturity model by defining relations between focus area maturity levels and improvement actions (type 5). 
The fifth type of theory in Gregor's taxonomy is termed design theory (Gregor and Hevner, 2013). This type of theory describes how to do something. Type 1 until 4 theory can be seen as descriptive knowledge. Type 5 as prescriptive knowledge.

The version of the maturity model and assessment instrument designed, used and evaluated in this thesis, results mostly in descriptive knowledge. Because we are using existing maturity models for the focus areas, the characteristics of the maturity levels can be used as a basis for defining improvement actions (prescriptive knowledge).

When complete and enriched with relations and recommended actions (see section 5.5 and 9.5) the model and instrument will incorporate substantial prescriptive knowledge.

\subsection{Research method}

This research uses qualitative research methods and design science research. The research was intended to design an instrument - in design science often referred to as an "artefact" - to measure and improve ITG in an organisation.

Design science research in IS addresses what are called wicked problems (Rittel and Webber, 1973). These represent problems that are characterised by complex interactions among subcomponents of the problem and with critical dependence upon human cognitive (e.g. creativity) or social abilities (e.g. teamwork) to produce effective solutions (Hevner et al., 2004). Such problems are ill-structured, with design theory constituting "a set of constructs and methods that enable the construction of models" to help managers and employees establish and evaluate alternative solutions (Pries-Heje and Baskerville, 2008).

Consultants in practice must base their approach on available ITG frameworks and experience. Scientific support based on a reliable maturity model would be very helpful.

The first step was searching existing ITG literature for usable frameworks or maturity models which includes the soft side. There are a lot of ITG frameworks but they tend to focus on the hard side (structure, process).

There might be usable frameworks in literature. To find out if maturity models or frameworks covering hard and soft ITG exists we conducted a systematic literature. Given that we could not find a maturity model for ITG that covers hard and soft governance, even though there is a need for such a model in research and practice, we designed an appropriate model in this research. Thus, another purpose of the systematic literature review was to find a starting point for the design of our ITG maturity model. 
The artefact we intend to design must combine organisational as well as behavioural and social elements, in our endeavours to cover hard and soft governance. In the next sections each research methodology component is briefly introduced.

\subsubsection{Systematic literature review}

Early research on ITG included contingency studies from the organisation sciences (Sambamurthy and Zmud, 1999, Brown, 1997). Method engineering provided frameworks and processes to assemble IS development methods from existing methodologies and inventories (Brinkkemper, 1996).

This research is partly based on previous research, and as such we conduct a systematic literature review, as used in IS and the social sciences (Petticrew and Roberts, 2006, Kitchenham, 2004). A systematic literature review is a methodologically rigorous review of research results. It is also intended to support the development of evidence-based guidelines for practitioners (Kitchenham et al., 2009).

Our systematic literature review (Petticrew and Roberts, 2006) on ITG was set up and conducted using Scopus and the Association for Information Systems (AIS) database. Scopus is the world's largest abstract and citation database and includes scholarly journals and book publishers including Wiley Blackwell, Springer Science \& Business Media, Taylor \& Francis, Sage Publications, Nature Publishing, IEEE and ACM. It also includes content from providers such as LexisNexis, Thomson Reuters (Web of Science), JSTOR, ARTstor, Credo Reference, Encyclopedia Britannica, World Book, ABC-CLIO, the HathiTrust Library and many others.

The search capabilities of AIS are less advanced, resulting in a less clean results set. After removing duplicates, the resulting set was manually selected by title and abstract. Author keywords were not available in the export files.

During the manual selection process, documents were selected that satisfied the following rules:

- $\quad$ The topic of the document must be ITG;

- Performance-related;

- Written in English, German or Dutch;

- $\quad$ Claims must be justified or based on research;

- $\quad$ Duplicated studies are excluded.

Systematic literature studies were conducted at the beginning (2012 and 2013) and end stages (2017 and 2018) of the research. 


\subsubsection{Design science}

The design-science paradigm has its roots in engineering and the sciences of the artificial (Simon, 1996). At its root it is a problem-solving paradigm. Charles and Ray Eames (1972) define design as "a plan for arranging elements in such a way as to best accomplish a particular purpose".

Design science is "a body of intellectually tough, analytic, partly formalizable, partly empirical, teachable doctrine about the design process" (Simon, 1988). The scientific view of design originates from the concepts found in Simon's (1996) seminal book The Sciences of the Artificial. Design science is a science of the artificial that involves searching for the means by which artefacts help achieve goals in an environment (Pries-Heje and Baskerville, 2008). The environment in this research is the organisation. The goal of this thesis is to design an artefact that can help the ITG of an organisation to grow in maturity to become more effective.

There is no widely accepted definition of design-science research (Iivari and Venable, 2009). The design-science paradigm embraces seemingly contradictory principles (Baskerville et al., 2015). Design and science share the same subject - in this thesis people and organisations - and produce artefacts, but their aims, methods and criteria are quite different (Galle and Kroes, 2014). Indeed, design is concerned with synthesis, whereas science is concerned with analysis (Simon 1996). This has resulted in a rich discussion around the process of designscience research, its artefacts and the role of theory.

In order to create a useful artefact to solve a practical problem, we follow the guidelines of Hevner et al. (2004) and Peffers et al.'s (2007) design-science research methodology process model. In addition, we applied the guidelines and three cycles of Hevner: the Relevance cycle, the Design cycle and the Rigor cycle (Hevner, 2007).

In our research, each cycle was covered:

1. We use Delphi panels - see next section - with practitioners to make our research and artefacts relevant for practice. To be relevant in practice, the artefacts must be easy to use and understood in practice.

2. The research covers a period of three years, with yearly updates of our artefacts. Evaluation is a key activity in design-science research (Venable et al., 2016). We collect information from users and in the case studies to validate and evaluate the artefacts. "The actual success of a maturity model is proved if it brings about a discussion on improvement among the targeted audience" (Lasrado et al., 2017).

3. The studies are based on previous research and scientific methods when adding, combining or improving components of the artefacts. 
Design science is a commonly used approach in IS research as well in the social sciences (Mettler, 2009). Our goal is to design an ITG maturity model that can be used to help organisations to grow in maturity and thereby become more effective. This affects organisational processes, structures and the collaboration between people (the employees). Thus, we need to combine IS research and the social sciences.

Hevner et al. (2004) note that the design-science paradigm seeks to extend the boundaries of human and organisational capabilities by creating new and innovative artefacts.

In addition to the model, we aim to develop an assessment instrument to determine the current status of an organisation's ITG. The model was named the MIG model (Maturity IT Governance) and the instrument was named the MIG assessment instrument. The research approach combines knowledge from literature and experts from practice to achieve both "problem relevance" and "research rigour" (Hevner et al., 2004).

The instrument is "necessary to determine how maturity measurement can occur" using the MIG model by "inclusion of appropriate questions and measures within this instrument" (De Bruin et al., 2005).

Tarhan et al. (2016) propose a distinction between the maturity model and assessment instrument because:

1. The model describes an improvement path while the instrument determines the status quo;

2. The instrument is not necessarily unique: there could be more assessment instruments based on the same maturity model e.g. an instrument for self-assessment and an instrument for use by (specialised) assessors;

3. The absence of a clear distinction may lead to flawed designs (Röglinger et al., 2012) and confusion (Van Looy, 2014).

A maturity assessment instrument can be used to measure the current maturity level of a certain aspect of an organisation in a meaningful way (Proença and Borbinha, 2016). Maturity assessments are highly complex specialised tasks performed by competent assessors, rendering it an expensive and burdensome activity for organisations (Proença and Borbinha, 2016). There is room for improvement by the provision of easy-to-use assessment guidelines (Röglinger et al., 2012). It is important to test both the model and instrument (De Bruin et al., 2005).

Empirically founded maturity models are rare (Lasrado et al., 2015). Design science is wellsuited to designing maturity models. The development of a maturity artefact should follow 
a design science approach as it gives a "methodological frame for creating and evaluating innovative IT artefacts" (Hevner et al., 2004). It is important to involve stakeholders throughout the process of design and thereafter (De Bruin et al., 2005, Lasrado et al., 2017).

Experts agree that design research involves designs that are clearly driven by underlying theories (Pries-Heje and Baskerville, 2008), in which theory and experience are engaged in generating new artefacts intended to change social and/or physical reality in purposeful ways. The goodness and efficacy of an artefact can be rigorously demonstrated via well-selected evaluation methods (Hevner et al., 2004, Basili, 1996, Kleindorfer et al., 1998, Zelkowitz and Wallace, 1998).

After each cycle the design of the MIG assessment instrument (MIG: Mature It Governance) was evaluated and improved. During the design cycles a balance must be found in constructing and evaluating the evolving design artefact. Both activities must be convincingly based on relevance and rigour (Hevner and Chatterjee, 2010). In the evaluation process we use Gregor et al.'s anatomy of a design theory (Gregor and Jones, 2007). They state that any design theory should include as a minimum: (1) the purpose and scope, (2) the constructs, (3) the principles of form and function, (4) the artefact mutability, (5) testable propositions, and (6) justificatory knowledge.

Specifying the first six components is sufficient to creating a model of an artefact. To be able to implement the artefact in a practical instrument, two additional components should be added: (7) principles of implementation and (8) expository instantiation (= a physical implementation of the artefact).

\subsubsection{Delphi method}

As a research method we combine design science with the Delphi method. The Delphi method may be characterised as a method for structuring group communication processes so that the process is effective in allowing a group of individuals (as a whole) to deal with a complex problem and obtain "the most reliable consensus of opinion of a group of experts" (Linstone and Turoff, 1975). There are several types of applications of the Delphi method in information systems research (Okoli and Pawlowski, 2004). In our research we use it to generate propositions and as construct validation.

The careful selection of participants is important. The quality and responses of a Delphi panel are as good as the experts that participate (Linstone and Turoff, 1975, Taylor-Powell, 2002). 
In order to accomplish this "structured communication", several elements should be provided:

- $\quad$ Feedback of individual contributions of information and knowledge;

- $\quad$ Assessment of the group judgement or view;

- $\quad$ Opportunity for individuals to revise views;

- $\quad$ Some degree of anonymity for the individual responses (Linstone and Turoff, 1975).

Technical details of the Delphi study

The efficiency of our workshops (see section 3.3.2) was increased by a supplemental group communication process (Linstone and Turoff, 1975). We used a Group Decision Support System (GDSS) to improve the effectiveness of the group meetings (Fjermestad and Hiltz, 2000). For this purpose we selected the innovative tool Spilter by Canast, which is a user-friendly, webbased GDSS (Spilter, 2014).

The participants had to respond to questions and statements using a laptop or tablet. There was no hierarchy or dominance; each opinion counted and could be recorded. Responses were anonymous to the rest of the group.

For example, when asked to rate the streams, we used the tool to show graphs of the responses and obtain a consensus before proceeding to the next step. For some questions, for example when asked to enter disciplines, responses were shared. Each participant was shown all responses instead of only his or her own. Participants could also add their own responses or adopt them from the group list. In advance we defined a maximum number of responses for each question. More technical details and an example of a screenshot can be found in Section 5.2.1.

\subsubsection{Maturity modelling}

Since the launch of CMM, hundreds of maturity models have been launched across a multitude of domains by researchers and practitioners (De Bruin et al., 2005, Weber et al., 2008). Maturity models are often modelled on CMMs, distinguishing a fixed number of (usually five) generic maturity levels. Examples in the literature are commonplace (Simonsson et al., 2010, Grant and Pennypacker, 2006, ITGI, 2007).

CMM also has its critics (Bach, 1995, Ngwenyama and Nielsen, 2003), who especially argue that it places too much emphasis on processes, and that in order to improve organisations, attention must be paid to other aspects such as people, culture or leadership as well.

Over time, some changes have occurred in CMM. Fraser and colleagues reviewed a number of maturity grid-based initiatives and concluded that "it is clear that many different approaches have been adopted" (Fraser et al., 2002). The aims of maturity models are "raising awareness" of 
what is going wrong, and "benchmarking" to compare results across organisations (Maier et al., 2012). Therefore, maturity models are helpful in finding better solutions for change. However, in order to be made useful, they must be applied to a substantial number of companies for valid comparison.

A "multi-theoretic approach" is required to determine the essential focus areas to improve organisational functioning (Daily et al., 2003). Becker et al. (2009) has analysed suitable procedures for the design of maturity models that comply with the guidelines for design science by Hevner et al. (2004). Our approach is based on previously applied research methods, such as by de Bruin and Rosemann (2007). The research in this thesis combines Multi-methods and Iterative procedures, supplemented with a few specific steps required to design a focus area maturity model.

A Focus Area Maturity Model (FAMM) is a specific type of maturity model in which an incremental improvement is based on the improvement to a collection of focus areas. FAMMs are discussed in detail in Section 5.5.1.

In our research we differentiate three subjects concerning ITG: hard governance, soft governance and the context. We need to determine which focus areas are relevant for each of these subjects (see Table 4).

\begin{tabular}{|l|l|}
\hline Subject & Focus area \\
\hline \multirow{2}{*}{ Hard governance } & Area 1 \\
\cline { 2 - 2 } & Area 2 \\
\hline \multirow{2}{*}{ Soft governance } & Area 3 \\
\cline { 2 - 2 } & Area 4 \\
\hline Context & Area 5 \\
\cline { 2 - 2 } & Area 6 \\
\hline
\end{tabular}

Table 4 Relevant focus areas for IT governance

Our multi-method iterative procedure to design a FAMM for ITG is based on literature research, the Delphi method, workshops, interviews and three cycles of iteration. 


\subsubsection{Case studies, cross-case synthesis and triangulation}

We follow the theoretical proposition that improving ITG focus areas will result in more mature ITG, and this will result in improved firm performance.

"Case studies are the preferred strategy, when 'how' or 'why' questions are being posed, when the investigator has little control over events, and when the focus is on a contemporary phenomenon in some real-life context" (Yin, 2013). The use of case studies is prevalent in business and IT management education to "highlight the complex business problems faced" by managers (Evans, 2016). ITG is an example of such a phenomenon. The assessment instrument was used in case studies conducted by students and by the researchers.

The reasons for choosing this combination are threefold:

First, we incorporated triangulation by using different methods to collect data: participants were asked to fill out the assessment instrument, participants were interviewed using the results sheet, and the case studies were conducted by both Dutch and international full-time student-groups and researchers. By cross-validating the instrument when used by students and more experienced researchers, we expect to acquire a better understanding of the usability of the MIG assessment instrument in practice.

The case study allowed students to bring topics together and support students to link and apply theory to practice (Race et al., 2004), as well as develop useful insights regarding the complex workings and functional interactions of an organisation (Brown and Knight, 2012, Jennings, 1996). We adopted Willcocksen's unusual two-way flow of activity and research-based teaching to improve learning outcomes for students and research outcomes for academic staff (Willcoxson et al., 2011).

Second, improving the research and education of Master's degree students registered for the IT management course at our university. This was a two-way process that "may be adapted to any discipline" and will lead to "both improved learning outcomes for students and improved research outcomes for academic staff" (Willcoxson et al., 2011). Studies on the nexus between teaching and research reveals that the variables used for teaching/learning quality or output and their operationalisation are both diverse and limited (Verburgh et al., 2007). Recent empirical evidence tends however to indicate a positive correlation between research performance and teaching (Artés et al., 2017). 
Students were enabled - but not required - to use the MIG assessment instrument to assess a medium- or large-size organisation (1000 FTE or more) in a practical group assignment. By summer 2018, none of the student groups had decided to use a different approach.

If they chose to use the instrument, the students were required to follow the case study protocol. By engaging Master's degree students registered for the IT management course in ITG research, we complete an unusual two-way relationship, in which research underpins teaching and learning, and the teaching and learning activity underpins research.

Third, the designed artefact was intended for use in practice. The assumption was that if students are able to use the instrument, it can be expected that practitioners - who in general have much more practical experience - will also be able to use it.

In order to facilitate the task of analysing the case study evidence, we created a case-study database (Darke et al., 1998). As a tool for the database we use Excel, which was also used to create the MIG assessment instrument. This enabled the simplest possible exchange of data between instrument and database.

One of the practical difficulties of the analysis of case study evidence is dealing with the amount and variety of the data. For the collection of data we used the MIG assessment instrument and interviews discussing the results. The use of an instrument greatly simplified the structuring of the data collection and the creation of the database. The data of each assessment, as well as all data collected during the steps of the case study protocol, were collected into the database.

We conducted case study research as an alternative to grounded theory-building through the synthesis of more structured approaches of qualitative data collection and analysis (Eisenhardt, 1989). For data collection we used the artefacts created with design science, combined with interviews. As an analytic technique, we used cross-case synthesis (Yin, 2013, Darke et al., 1998).

In our research we covered each of the four validity and reliability criteria: internal validity, construct validity, external validity, reliability (Cook et al., 1979), including as far as possible the relationships among them (Gibbert et al., 2008). 
Table 5 presents a summary of the link to the design cycle in this research:

\begin{tabular}{|l|l|}
\hline Criterium & Link to the design cycle \\
\hline Internal validity & $\begin{array}{l}\text { Internal validity or the extent to which the results of the research support a claim } \\
\text { about cause and effect. } \\
\text { This is linked to the design cycle by: } \\
\text { Theory triangulation (e.g. the combination of hard and soft ITG); } \\
\text { Combining literature research, case study research using assessments and } \\
\text { interviews; } \\
\text { Evaluations with all people involved (e.g. participants); } \\
\text { Conducting multiple design cycles. }\end{array}$ \\
\hline Construct validity & $\begin{array}{l}\text { Construct validity defines how well the research measures what it claims. } \\
\text { This is linked to the design cycle by: } \\
\text { The use of data triangulation (e.g. the combination of assessments with } \\
\text { interviews); } \\
\text { Using literature and practitioners do design the model and the instrument. }\end{array}$ \\
\hline External validity & $\begin{array}{l}\text { External validity or the extent to which the results of a case study can be applied } \\
\text { beyond the context of the study. } \\
\text { This is linked to the design cycle by: } \\
\text { Details on the context of the case are included into the MIG model; } \\
\text { Cross-case analysis (e.g. for the focus area "informal organisation"). }\end{array}$ \\
\hline Reliability & $\begin{array}{l}\text { Reliability is the extent to which the results are consistent. } \\
\text { This is linked to the design cycle by: } \\
\text { Using case study protocol; } \\
\text { Using a case study database. }\end{array}$ \\
\hline
\end{tabular}

Table 5 Link between the four validity and reliability criteria and the design cycle

\subsection{Research model}

Our research is based on a simple research model linking the ITG focus areas and context to ITG maturity and ITG effectiveness (see Figure 3).

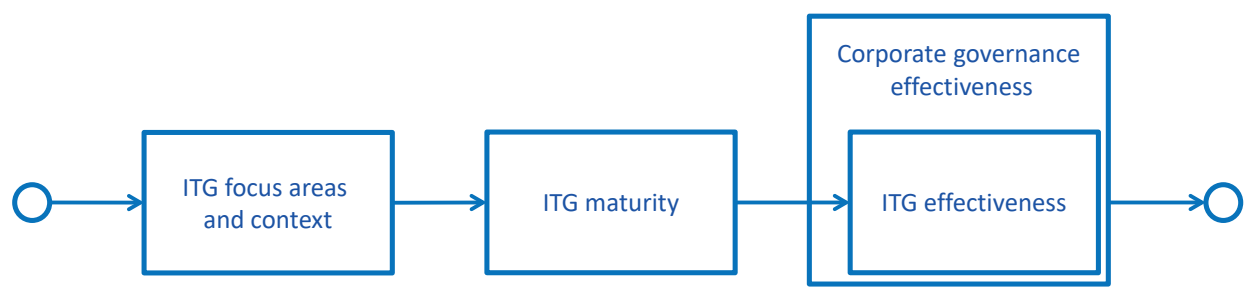

Figure 3 Research model

The starting point was the rationale behind the research, represented as the circle on the left. The research model depicts a link between ITG maturity and ITG effectiveness. Our research 
focuses on performance as we relate ITG effectiveness to organisational performance. In order to bridge the gap between the performance of ITG and the performance of the organisation, we consider ITG part of corporate governance (similar with stream 4 in Table 1). Organisational value is created by corporate governance effectiveness, and corporate governance effectiveness is (partly) created by ITG effectiveness.

The end point - the circle on the right - is the answer to the main research question and the conclusions.

\subsubsection{Research questions}

Combining the research questions represented in Table 6 with the research model discussed in the previous section results in Table 6 .

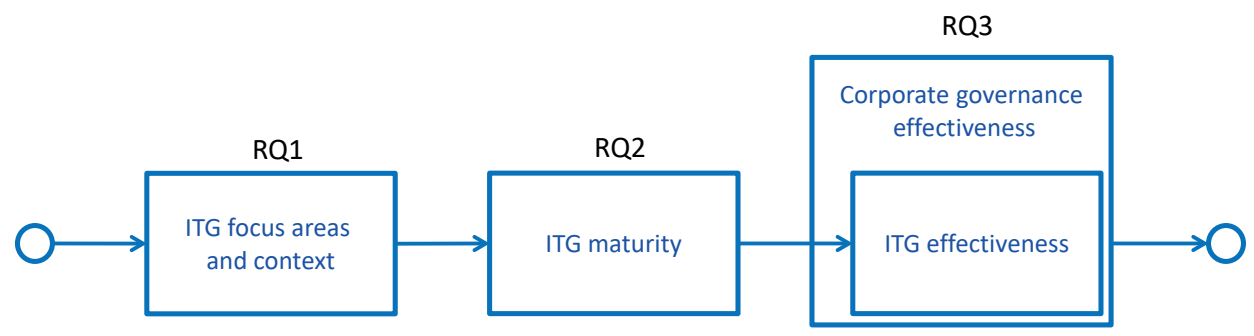

Figure 4 Research model including research questions

The main research question (MRQ), described in Section 2.1, was broken down into three research questions:

[RQ1] What practices constitute IT governance?

[RQ2] How can the IT governance of an organisation grow in maturity?

[RQ3] How can we measure the IT governance maturity of an organisation?

The answer to the first research question results in a division of ITG into several focus areas. These focus areas are used in the second research question (arrow). The second research question concerns ITG maturity. This research question is answered by determining how the ITG focus areas found in the rectangle mature.

The third research question is about ITG effectiveness. As described in Section 1.4, this research is rooted in the assumption that improving "ITG maturity" results in improving ITG, and because ITG is an integral part of corporate governance, it also helps improve organisational performance. This research question is broken down into sub-questions for the design of an 
instrument to measure ITG maturity that can be used to improve ITG maturity (and thus ITG effectiveness) and as illustration of the relevance of the results for corporate governance.

This thesis consists of an introductory section followed by eight sections that are partly based on published research papers.

The sections are grouped in five parts, as shown in Figure 5

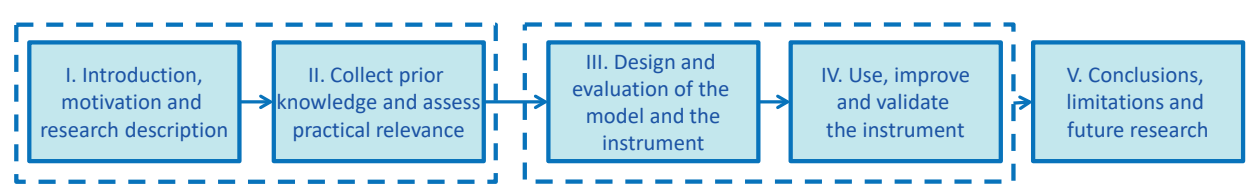

Figure 5 Structure of the thesis

The first part consists of an introduction to the research and the research methods. In the second part, prior research is collected, summarised and discussed with practitioners to determine the relevance and requirements for the next phase. The results of Part II will be used to design a focus area maturity model and a maturity assessment instrument (Part III). Part IV describes three cycles in which consecutive versions of the assessment instrument are used, evaluated, validated and improved in practice, along with a systematic literature study on the relationship between corporate governance and ITG. Part V consists of the answers to the research questions, conclusions, limitations and further research.

Thus, the five steps followed in this thesis are:

1. Introduction and motivation;

2. Research questions and method (this section);

3. Collect prior research knowledge;

4. Check relevance with practitioners;

5. Design of the MIG model.

The structure of the thesis and these five steps are described in detail in Section 2.4.

The research questions were broken down into sub-questions and can be related to the steps followed in this thesis (see Table 6). 
Section 2

\begin{tabular}{|c|c|c|c|}
\hline \# & Step & \multicolumn{2}{|c|}{ Research Question } \\
\hline I. & $\begin{array}{l}\text { Collect prior } \\
\text { research } \\
\text { knowledge }\end{array}$ & $\begin{array}{l}\text { [RQ1] } \\
\text { This res } \\
\text { question } \\
\text { [SQ1.1] }\end{array}$ & $\begin{array}{l}\text { What practices constitute IT governance? } \\
\text { arch question is broken down into the following related sub- } \\
\text { Which stream(s) of IT governance best align with current } \\
\text { practices? }\end{array}$ \\
\hline II. & $\begin{array}{l}\text { Check } \\
\text { relevance with } \\
\text { practitioners }\end{array}$ & $\begin{array}{l}\text { A seconc } \\
{[\mathrm{SQ} 1.2]} \\
{[\mathrm{SQ} 1.3]} \\
\text { [SQ1.4] }\end{array}$ & $\begin{array}{l}\text { sub-question related to the first research question is: } \\
\text { According to practitioners, which disciplines should play an } \\
\text { important role in IT governance? } \\
\text { Which (new) ITG maturity models are available in the literature } \\
\text { that can be used in practice? } \\
\text { Is there (still) a mismatch between IT governance practice and } \\
\text { theoretical frameworks? }\end{array}$ \\
\hline III. & $\begin{array}{l}\text { Design the } \\
\text { instrument }\end{array}$ & $\begin{array}{l}\text { [RQ2] } \\
\text { This rese } \\
\text { Designin } \\
\text { [SQ2.1] } \\
\text { [SQ2.2] } \\
\text { [SQ2.3] } \\
\text { Designin } \\
\text { [SQ2.4] } \\
\text { [SQ2.5] }\end{array}$ & $\begin{array}{l}\text { How can the IT governance of an organisation grow in maturity? } \\
\text { rch question is divided into the following related sub-questions: } \\
\text { the MIG model: } \\
\text { Which focus areas should an ITG maturity model for soft and hard } \\
\text { governance contain? } \\
\text { What type of maturity model do we need? } \\
\text { What are the capabilities of each focus area? } \\
\text { the MIG assessment instrument: } \\
\text { an can the current hard and soft IT governance be measured in } \\
\text { How can we develop an assessment instrument based on the MIG } \\
\text { model? }\end{array}$ \\
\hline IV. & $\begin{array}{l}\text { Use, improve } \\
\text { and validate } \\
\text { the instrument }\end{array}$ & $\begin{array}{l}\text { [RQ3] } \\
\text { This rese } \\
\text { question } \\
\text { [SQ3.1] } \\
\text { [SQ3.2] } \\
\text { [SQ3.4] } \\
\text { [SQ3.5] }\end{array}$ & $\begin{array}{l}\text { How can we measure the IT governance maturity of an } \\
\text { organisation? } \\
\text { rch question is broken down in the following related sub- } \\
\text { How can we use the MIG assessment instrument to measure } \\
\text { current hard and soft ITG? } \\
\text { How usable is the instrument for measuring current hard and soft } \\
\text { IT governance? } \\
\text { How usable are the ITG focus areas for corporate governance } \\
\text { research? } \\
\text { How usable is version } 3 \text { of the assessment instrument for } \\
\text { measuring corporate governance in an organisation? } \\
\text { How do ITG, corporate governance and organisational } \\
\text { effectiveness relate? }\end{array}$ \\
\hline
\end{tabular}




\begin{tabular}{|l|l|l|}
\hline$\#$ & Step & Research Question \\
\hline V. & $\begin{array}{l}\text { Conclusions } \\
\text { and future } \\
\text { research }\end{array}$ & $\begin{array}{l}\text { HRQ] } \\
\text { How can the ITG of an organisation grow in maturity to become } \\
\text { more effive? }\end{array}$ \\
\hline
\end{tabular}

Table 6 Research questions and sub-questions per step

This thesis represents one of the results of this doctoral research. The structure of the thesis is described in the following section.

\subsection{Thesis structure}

The thesis is structured in five parts. Part I of the thesis constitutes Section 1 Introduction to the research and this section (see Figure 5).

A short explanation of each part of the thesis:

Part I. Introduction, motivation and research description

1. Introduction and motivation

The first section contains an introduction to ITG and ITG maturity, as well as the motivation for the research.

2. Research questions and method (this section)

This section contains an introduction to the research methods used and an overview of each research step.

Part II. Collect prior research knowledge and assess practical relevance

3. Collect prior research knowledge

ITG is focused on information technology (IT) and an integral part of corporate governance (ITGI, 2003b, Van Grembergen et al., 2004). In this research we collect prior research knowledge for both ITG and corporate governance.

The rising interest in ITG is partly due to compliance requirements. Today almost every large organisation is more or less dependent on IT. As aresult, the impact of IT on performance is growing. ITG is emerging as an important area of inquiry among academics and practitioners alike. The goal of the research is to add to existing knowledge on how to implement and professionalise the ITG of organisations. 
Section 2

4. $\quad$ Check relevance with practitioners

During this step practitioners are questioned regarding the relevance of the research and the most important focus areas. Several different research methods are used.

This second step will result in a conclusion regarding the relevance of the research.

Part III: Design of the model and the instrument

5. Design of the MIG model

In meetings with practitioners we use design science and Delphi sessions to design an ITG maturity model for hard and soft governance.

The result of this step was an ITG focus area maturity model (FAMM): the MIG model.

6. Design of the MIG assessment instrument

During this third step we use design science and Delphi sessions to design the instrument. Existing knowledge will be used and supplemented with the collected requirements and insights from practitioners.

The result of this step was (a first version of) an ITG assessment instrument based on the MIG model: the MIG assessment instrument.

Part IV: Use, improve and validate the instrument

7. The design cycles

In the first section of Part IV we describe three design cycles of the MIG assessment instrument. Section 7.3 describes the results and evaluation of the initial version, Section 7.4 the second version and Section 7.5 the third version.

a. The first cycle and case studies in 2015

Following the design of the initial version of the MIG assessment instrument, several case studies were conducted by students and the researchers. After analysing the results of the case studies and the evaluation by the users, a list of possible improvements was created.

b. The second cycle and case studies in 2016

For the second cycle a selection of the suggested improvements were implemented,resulting in a second version of the instrument. This is because "in fact, the more the format, order, wording, and procedural setting of the 
original instrument is changed, the greater the likelihood that the derived instrument will lack validated qualities of the original instrument" (Straub, 1989). This means that we must be careful when changing the instrument. In fact, each year we changed the instrument only minimally for obvious inadequacies or improvements suggested by a substantial part of the participants. Having altered the instrument at the beginning of the year, it remained unchanged for the remainder of the case studies conducted in 2016.

After analysing the results of the second cycle of case studies, comparing results with the first cycle and collecting the evaluations of the users, an update of the list with possible improvements was created.

c. The third cycle and case studies in 2017

During the third and final cycle (described in this thesis), the process was the same as in the second cycle. Based on the evaluation of the second cycle the instrument was changed again at the beginning of the year. The instrument was used in another series of case studies and remained unaltered during the rest of the year.

8. The link between corporate governance and IT governance

Corporate governance and ITG are related. However, little is known about the ways in which this relationship works. This section describes the results of a systematic literature review on corporate governance to determine if the focus areas of the MIG model are covered in corporate governance literature, and hence if the MIG model can be used for corporate governance, too.

Part V: Conclusions, limitations and future research

9. Conclusions limitations and future research

After creating the initial version, the instrument was used, improved, evaluated and validated in three cycles. Given that we were cautious when changing the instrument, we were able to analyse the results of the case studies in comparison with one another but also between cycles. The end results of this analysis are summarised in this section alongside the conclusions and recommendations. This section also provides an answer to the main research question, limitations and next steps. 


\subsubsection{Research methods per step}

The (main) research methods used in each step is summarised in Table 7.

\begin{tabular}{|l|l|l|}
\hline$\#$ & Step & Research Method \\
\hline I. & Collect prior research knowledge & Systematic literature review \\
\hline II. & Check relevance with practitioners & Delphi panels \\
\hline III. & Design the instrument & $\begin{array}{l}\text { Design science } \\
\text { Delphi panels } \\
\text { Maturity models }\end{array}$ \\
\hline IV. & Use, improve and validate the instrument & Case studies, cross-case synthesis and triangulation \\
\hline V. & Conclusions and future research & \\
\hline
\end{tabular}

Table 7 Research methods per step

\subsubsection{Research activities and results}

The timeline of the approach of the research activities was as follows:

2013 Systematic literature study.

2014 Design of the MIG model and instrument.

2015 First cycle using the MIG instrument.

2016 Second cycle using the MIG instrument.

2017 Third cycle using the MIG instrument.

2018 Update literature study and writing this thesis.

A more detailed overview of the research activities and results conducted between 2013 and 2018 is shown in Figure 6.

For the sake of clarity, not all activities conducted are included in the Figure. Furthermore, not all activities took place in the exact sequence as shown, or occurred after the end of the year.

Figure 6 is used as an outline for the research.

In each section this Figure is used to provide an overview of the activities and results discussed in that section. Each activity and result will be described in detail. Table 8 includes an overview of the parties involved in each research activity. 
Research questions and method

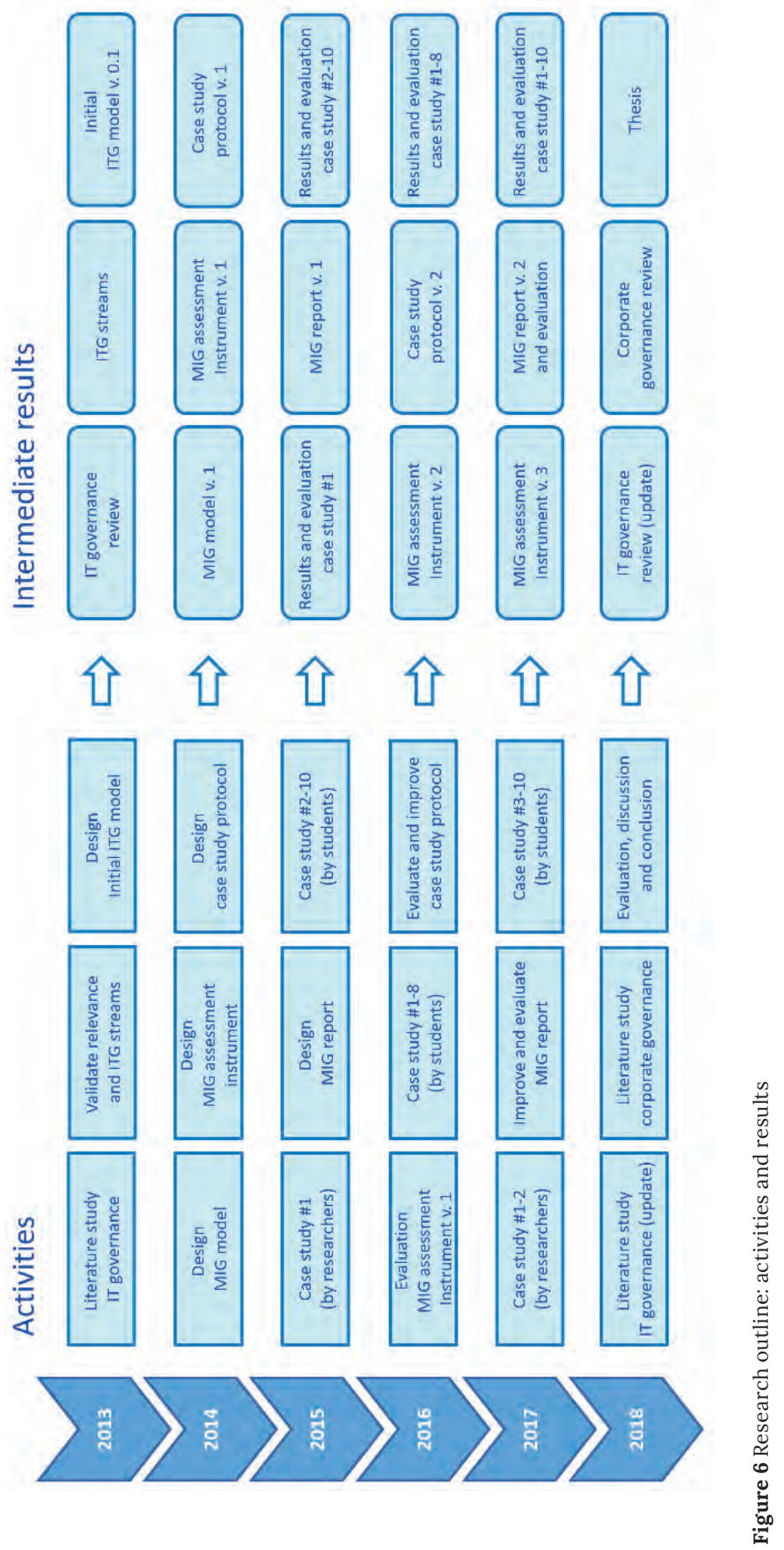


Section 2

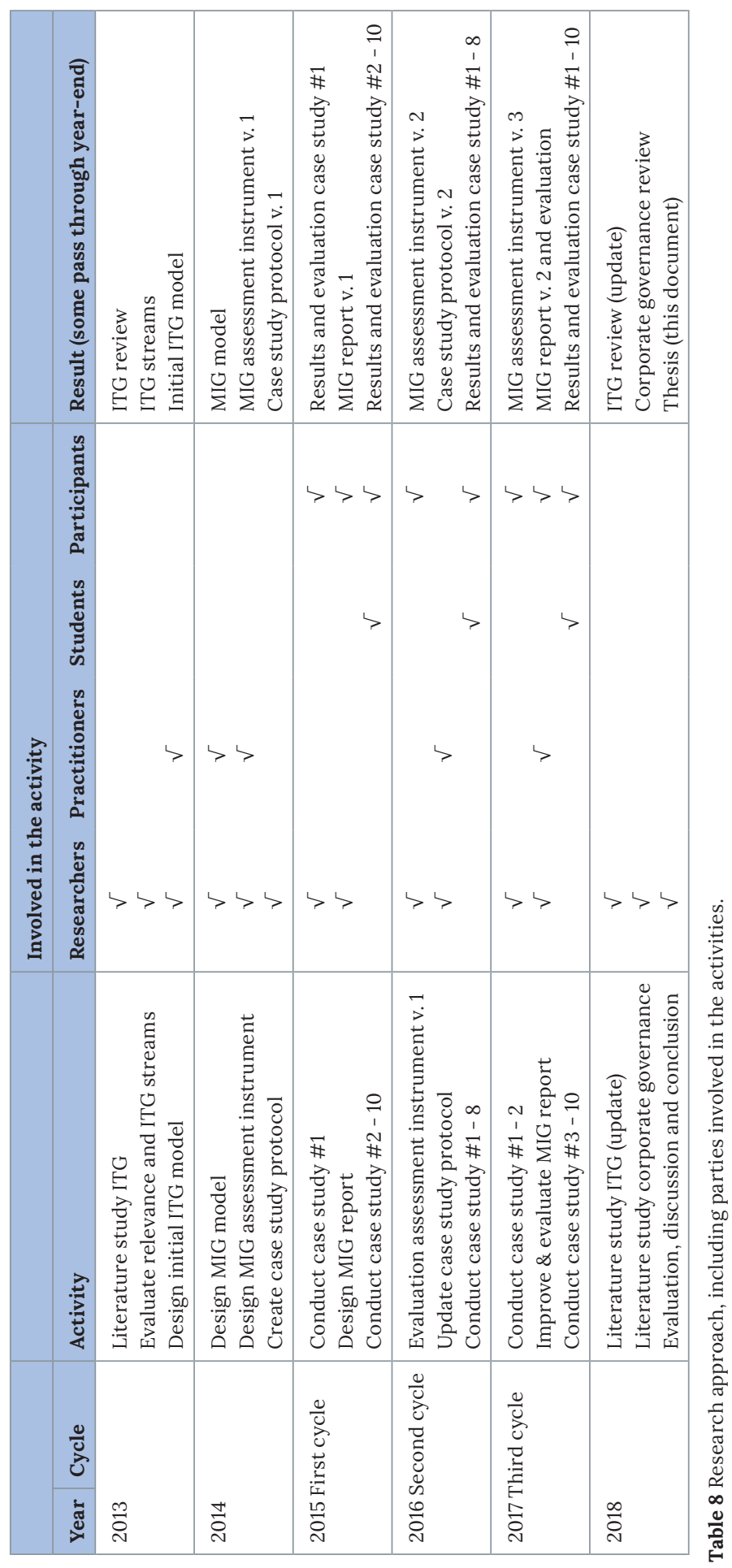


A short introduction of each of the parties:

- $\quad$ Researchers: the researchers are Daniël Smits (principal investigator) and Jos van Hillegersberg;

- Practitioners: a diverse group of experienced participants from practice. The composition of the group is described in each study;

- $\quad$ Students: full-time Master's degree students registered for the IT management MSc course at the University of Twente, and part-time MBI students at Avans+ University of Applied Sciences;

- $\quad$ Participants: the corporate or government participants in the case studies.

\subsection{Summary of this section}

The goal of this thesis is to determine how the ITG of an organisation can grow in maturity to become more effective. This section provides a detailed description of the research methods, the research questions and the research model. The research method is based on design science and a combination of systematic literature studies, Delphi workshops and case studies.

Section 2.4 contains an overview of the structure of the thesis and a research outline (Figure 6). The research outline will be used in subsequent sections to clarify the elements (activities and results) described in each section and how they relate to the other activities and results.

The current section represents the final section of the first part of the thesis: "Introduction, motivation and research description".

The next part is entitled "Collect prior knowledge and assess practical relevance". It describes a systematic literature review on ITG and a Delphi workshop with a group of CIOs. 


\section{Part I I}

\section{Collect prior knowledge and assess practical relevance}

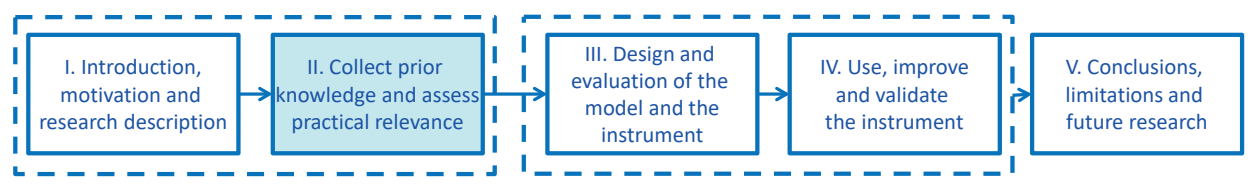

Figure 7 Thesis Part II. Collect prior knowledge and assess practical relevance

\section{Section}

3. Collect prior research knowledge and assess practical relevance

4. Systematic literature review on ITG: 2018 update 


\section{Section 3}

\section{Collect prior research knowledge and assess practical relevance}

\subsection{Introduction}

In Step II, prior research knowledge is collected and the practical relevance of the research is determined. During this step several studies and activities have been conducted (marked in light blue in Figure 8).

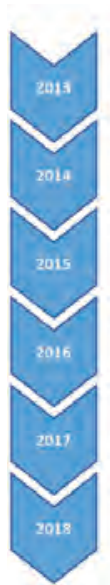

Activities
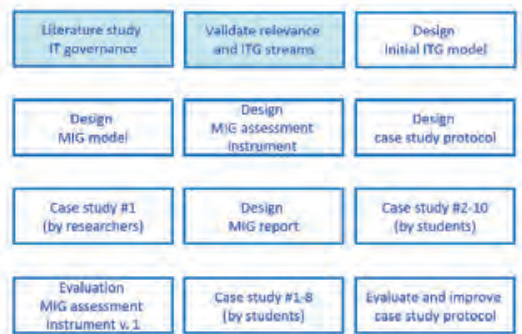
case study protocol
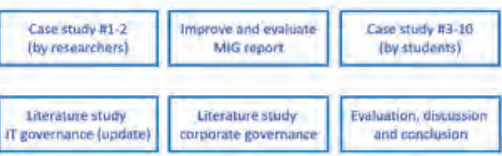

Literature study IT governance (upidate) corporate governange

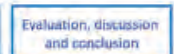

Intermediate results
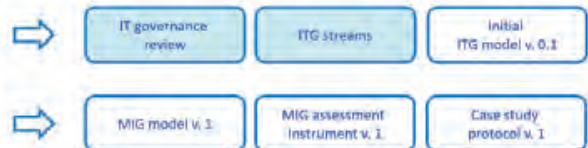
instrument $x .1$

Case study protocol i. I

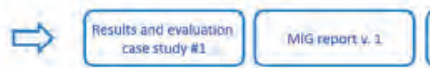

Resuits and evaluation case study $=2-10$

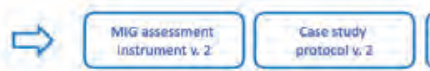

Rults and evaluation case study स1-a
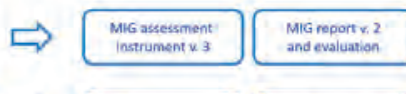
and evaluation
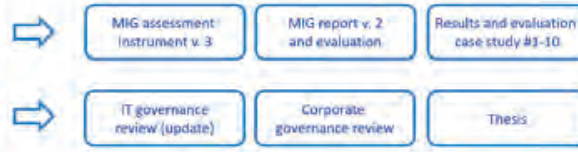

Figure 8 Activities and results of Step II, Part A.

The first set of the activities and results (the light blue elements) are discussed in this section. The research methods for these activities has been described in Section 2. Further details regarding the approach and results of the activities are described in this section. The content here is based on previously published work. ${ }^{3},{ }^{4}$

3 Smits, D. \& van Hillegersberg, J. 2013. The continuing mismatch between IT governance theory and practice: Results from a Delphi study with CIO's. 19th Americas Conference on Information Systems (AMCIS), Chicago, IL. AIS Electronic Library, pp 3278-3286.

4 Smits, D. \& van Hillegersberg, J. 2014a. The continuing mismatch between it governance theory and practice: Results from a systematic literature review and a delphi study with cio's. Journal of management systems, Vol. 24, No. 3, pp 1-20. 
The first step in this research was searching existing ITG literature for usable frameworks or maturity models that include the soft side. There are a lot of ITG frameworks but they tend to focus on the hard side (structure, process). There might be usable frameworks in literature. To find out if maturity models or frameworks covering hard and soft ITG exists we conducted a systematic literature review.

Selecting papers related to soft governance in a systematic way is difficult because it is not very clear which keywords should be used. We expected the use of social-related phrases like behaviour, culture or collaboration. This however means that the selection found might not be complete. The keywords we used were "behavior", "behaviour", "collaborate", "collaboration", "culture" and "social".

Given that we could not find a maturity model for ITG that covers hard and soft governance, another purpose of the systematic literature review was to find a starting point for the design of our ITG maturity model.

Thus, the purpose of the systematic literature review was:

a. find out if maturity models or frameworks covering hard and soft ITG exists in literature;

b. $\quad$ find a starting point for the design of our ITG maturity model;

c. to create an initial list of relevant focus areas and corresponding maturity models to design the initial version of a focus area maturity model for ITG.

The discussion of the questions in the sections of this thesis are not always in the most logical order. This is because the papers have already been published and used afterwards for this thesis. The idea was to keep the papers partly intact and replace the introduction and conclusion to improve the readability of the sections.

Our initial proposition of the relevance of architecture and portfolio management was confirmed by the CIOs (this Section), but as will be shown in Section 5 , this did not reappear in the Delphi workshops when determining the focus areas in the design process of the ITG maturity model. For that purpose the keys "architecture" and "portfolio" management are included in the systematic literature review.

Given that these disciplines did not appear in the results from the Delphi sessions when designing the MIG instrument, we did not pursue research on these disciplines in this thesis. 
In this section we describe the results of the following two activities:

- $\quad$ A systematic literature review (Petticrew and Roberts, 2006) on ITG was established and conducted in 2013;

- $\quad$ A Delphi study with members of the CIO Platform Netherlands (CIOPN). Members of CIOPN are responsible for an IT budget of $€ 25$ million or greater.

The systematic literature review was repeated in 2018 for ITG. Details regarding this update are included in Section 4.

\subsection{Research questions}

The goal of this thesis is to determine which streams in the existing ITG literature best align with current practices and which disciplines and frameworks are used for ITG. A further goal is to collect indications on how to improve ITG using literature and experts from practice. In literature, constructs such as dimensions, focus areas or principles are often used to refine the concept of ITG. Dimensions may include IT compliance management or business/ IT alignment (Novotny et al., 2012); examples of focus areas are value delivery or resource management (ITGI, 2003b); and principles could be strategy or responsibility (ISO/IEC 38500, 2008). For applicability in practice we use the more practical term "discipline". Examples of disciplines are architecture or portfolio management, which directly relate to roles or functions in an organisation, such as architect or portfolio manager.

Thus, the goal of this section is to answer the following questions:

SQ 1.1: Which stream(s) of ITG best align with current practices?

SQ 1.2: According to practitioners, which disciplines should play an important role in ITG?

SQ 1.4: Is there (still) a mismatch between ITG practice and theoretical frameworks?

The final question was added because we expect a mismatch and are seeking new or innovative ways to improve ITG. Another important goal was to collect indications on how to improve ITG using experts from practice and literature.

\subsection{Research method}

The research methods applied in this section are based on a combination of a systematic literature review and a Delphi study using the Spilter Group Decision Support System (GDSS). 
Our research process started by exploring the research domain through a systematic literature review in the domain of ITG. As a subsequent step we conducted a Delphi study in a meeting with a group of Dutch CIOs.

\subsubsection{Systematic Literature set-up}

A systematic literature review (see Section 2.2.1) on ITG was established and conducted using Scopus and the Association for Information Systems (AIS) databases.

In recent papers, ITG is occasionally referred to as "corporate governance of IT', as in ISO/IEC 38500 (Calder, 2008), while others use "enterprise governance of IT" or "enterprise governance" (Dietz and Hoogervorst, 2012, Van Grembergen and De Haes, 2010). Moreover, in some papers ITG is called "IS governance", in which "IS" stands for "information systems" management (Brown and Renwick, 1996). In the period of August 2012 to September 2012, we made an initial selection of documents in Scopus searching for ITG in the title, abstract or author keywords. For our selection we used the keywords "IT governance", "governance of IT", "IS governance" and "enterprise governance".

The use of additional keywords also resulted in the selection of a substantial set of nonIT-related corporate governance or enterprise governance documents, which were excluded during the selection process.

After analysing the set we noticed that we were missing important documents. In the period of April 2013 to May 2013 we thus updated our set from Scopus and used the same keywords in the eLibrary of the AIS. The AIS is a worldwide professional association with a large eLibrary of journal and conference papers that are not completely covered by Scopus.

The search capabilities of AIS are less advanced, resulting in a less clean result set. After removing duplicates, the resulting set was manually selected by title and abstract. Author keywords were not available in the export files. During the manual selection process documents were selected that satisfied the following rules:

- $\quad$ The topic of the document must be ITG;

- $\quad$ Performance-related;

- $\quad$ Written in English, German or Dutch;

- $\quad$ Claims must be justified or based on research;

- $\quad$ Duplicated studies are excluded. 
In order to collect indications on how to improve ITG from the literature, we also broke down three subjects that might deliver clues on new or innovative ways to improve ITG.

1. First, we were interested in ITG maturity as our research focuses on improving ITG effectiveness and maturity.

2. Second, we were interested in the disciplines of architecture and portfolio management. Recent literature presumes that linking ITG with these disciplines can greatly improve IT efficiency and IT effectiveness (Wittenburg et al., 2007, Niemann, 2006).

3. Third, we were interested in documents related to social aspects. Contemporary literature is often based on structural, top-down, planning processes. Critics argue that social aspects like culture, behaviour and collaboration deserve greater attention (more on this in the next section).

In order to select papers related to ITG maturity, we used the keywords "maturity" or "mature" to search in the title, abstract or author keywords. To select papers related to the disciplines of architecture and portfolio management we used the keywords "architecture" and "portfolio". Selecting papers related to social aspects in a systematic way is more difficult because it is less clear which keywords should be used. We expected the use of social-related phrases like behaviour, culture or collaboration. This however means that the selection found might not be complete. The keywords we used were "behavior", "behaviour", "collaborate", "collaboration", "culture" and "social".

A subsequent manual selection was undertaken to determine if the document was in scope. To be in scope the document had to satisfy the additional rule that the topic of the document is ITG, in relation to the relevant keywords.

\subsubsection{Delphi study set-up}

A Delphi panel was established (see Section 2.2.3) to generate propositions and to be used as a construct validation. The constructs in this thesis comprised the six ITG streams (see Table 12).

Careful selection of participants is important. Earlier research by the IT Governance Institute (ITGI, 2008) has indicated that CIOs and IT management are the best parties to ask questions concerning ITG: "Although championship for ITG within the enterprise comes from the C-level, in daily practice ITG is still very much a CIO/IT director issue" (ITGI, 2008).

With 14 participants, we also complied with the next guideline: "Ten to 15 people may be adequate for a focused Delphi where participants do not vary a great deal." (Taylor-Powell, 2002). 
The Delphi study was conducted during a meeting of the CIO Platform Netherlands (CIOPN). The CIOPN counts more than 100 members and provides a good reflection of the Dutch situation within the government and private enterprises. To become a member of the CIOPN an organisation must have at least 1,000 employees, have a sales volume of €500 million and/ or an IT budget of more than $€ 25$ million. For governmental organisations, no specific sales volumes are required.

Different people often have different understandings of the same concept. To address this we first presented, explained and discussed definitions of ITG. During this presentation we also introduced the six identified ITG streams.

For the Delphi study we used the web-based GDSS tool Spilter (see Section 2.2.3).

\subsection{Results of the study}

\subsubsection{Results of the systematic literature review}

The first selection between August and September 2012 using Scopus resulted in a set of 484 documents. During the second selection between April and May 2013 using Scopus an additional 169 documents were found (see Table 9).

\begin{tabular}{|l|l|l|l|}
\hline Library & Documents & $\#$ & \% \\
\hline \multirow{3}{*}{ Scopus } & $1^{\text {st }}$ selection (8/2012-9/2012) & 484 & \\
& $2^{\text {nd }}$ selection (4/2013-5/2013) & 169 & + \\
\cline { 2 - 4 } & Total selected & 665 & \\
& Remove duplicates & 6 & - \\
\cline { 2 - 4 } & Total excl. duplicates & 659 & \multirow{2}{*}{100} \\
\hline \multirow{2}{*}{ AIS } & In scope & 269 & \multirow{2}{*}{41} \\
\hline Total & Added (4/2013-5/2013) & 62 & + \\
\hline
\end{tabular}

Table 9 Totals systematic literature review 2012/2013

After removing duplicates, a result set of 659 documents remained. When applying the selection criteria listed in Section 3.3.1, the result set decreased to 269 documents. A manual selection on AIS using the same criteria produced 62 additional documents.

In summary, after completing the first and second analysis, the systematic literature review resulted in a set of 331 documents in scope. The analysis of the resulting documents will be repeated several times in the future. 
Breakdown by subject

The breakdown of the result set into subsets for the three subjects is summarised in Table 10.

\begin{tabular}{|l|l|l|}
\hline Documents (total \# selected = 331) & $\#$ & $\%$ \\
\hline On "maturity" or "mature" & 22 & 7 \\
\hline On "architecture" & 25 & 8 \\
On "portfolio" management & 14 & 4 \\
On "architecture" or "portfolio" management & 35 & 11 \\
\hline On "collaborate" or "collaboration" & 5 & 2 \\
On "behavior" or "behaviour" & 6 & 2 \\
On "culture" & 15 & 5 \\
On "social" & 7 & 2 \\
On all social-related keywords & 33 & 10 \\
\hline
\end{tabular}

Table 10 Documents on ITG grouped by specific topics

The numbers included in the table are the totals following manual selection to determine if the document was in scope.

A summary of the year of publication of the documents is shown in Table 11.

The percentages are the number of documents in a year compared to the number of documents in the same year in the complete set.

The complete set

The oldest documents in our complete set of 331 documents were from 1995, but the vast majority were from 2006 or later. Documents from 2013 were sparse because of the time of the selection and the fact that it always takes some time before publications are added to databases.

The subject of maturity

Using the keywords "maturity" or "mature" we found 30 unique documents. From this set, 22 documents were in scope. This set also included papers minimally discussing the topic. Regarding ITG maturity, the COBIT framework was used most often in contemporary research papers. The maturity model of COBIT is based on the Capability Maturity Model (CMM). Other maturity models were only found when focusing on other ITG perspectives, and these maturity models were also largely based on CMM. Documents on ITG maturity are relatively new, with the first dating from 2007/2008. 


\section{Section 3}

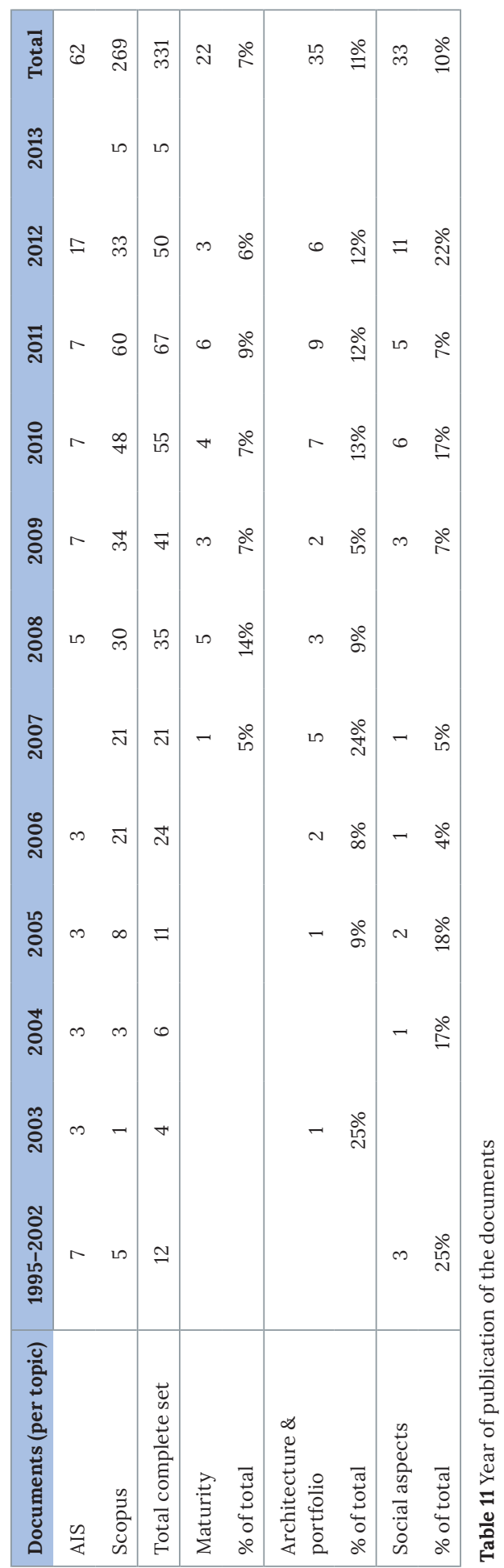


The subject of disciplines

Using the keywords "architecture" or "portfolio" (management), we found 43 unique documents. From this set 35 documents were in scope. We also included papers that discuss the topic minimally.

Table 10 shows the number of selected documents related to the disciplines "architecture" and "portfolio" (management) individually and with both disciplines combined.

The list of documents mainly consists of case studies (14), models or frameworks (9), a few surveys (4) and several other more specific types of papers (8). Some of the more interesting papers for the purpose of this research include a case study at the BMW group (Wittenburg et al., 2007), a paper discussing "enterprise engineering" relevant to the "bottom-up" view (Dietz et al., 2012) and a paper on quantitative portfolio management reporting anomalies or ambiguous ITG rules (Verhoef, 2007).

As can be seen in Table 10, documents that combine ITG with architecture and portfolio (management) are mostly relatively new. The number of publications started to grow from 2006/2007, just like the rest of the set.

The subject of social

Using the keywords "collaboration", "behavior", "culture" or "social" we found 39 unique documents. From this set, 33 documents were found to be in scope. We again included papers discussing the topic minimally.

This set of documents was very diverse, although the documents might contain indications on how to improve ITG because they use a distinctive perspective on ITG. Some of the more interesting papers for the purpose of this research discuss the relationship between ITG and Chinese culture (Zhong et al., 2012), user-driven innovation or "shadow IT" (Györy et al., 2012) and "entrepreneurial behaviour" influencing ITG (Bradley and Pratt, 2011).

Documents on social aspects were written in all periods examined and could be found in the complete period as with the complete set of documents.

The initial model for ITG streams

Scholars have different opinions regarding definitions of ITG. In our literature review we identified six streams based on two views:

1. The first viewing angle handles the scope of ITG;

2. The second viewing angle handles the direction in which ITG works. 
Section 3

Scope of ITG

Different streams can be distinguished. In particular, some scholars use a small scope, while others a broad scope. Weill and Ross (2004) use a small scope, defining ITG as "the decision rights and accountability framework for encouraging desirable behaviour in the use of IT". These authors can be viewed as the main contributors to the stream that focuses on decision-making, considering ITG from a decision-making perspective. As components of ITG, this stream uses elements such as IT decisions or decision archetypes (Weill and Ross, 2004, 2005). Others complement this with the context in which the decision is made (Xue et al., 2008).

A clear proponent of the broad definition is the ITGI. The ITGI is also the founder of COBIT, the well-known ITG framework (as of version 4.0) formerly known as Control Objectives for Information and Related Technology. However, ITIL or ISO 20000 are currently the most frequently mentioned external frameworks used as a basis for ITG (ITGI, 2011). Some researchers define ITG "as the process by which decisions are made around IT investments" and claim that ITIL version 3 can provide a mature framework for ITG (Nabiollahi and Sahibuddin, 2008).

In this research we use three streams of ITG as a starting point: IT auditing, decision-making and ITG as an integral part of corporate governance (Musson, 2009).

Research has indicated that "structure" and "process" are the basic elements of corporate governance's institutional dimension (Nerantzidis et al., 2012), with these authors demonstrating that almost every definition of corporate governance refers to them. At one end of the continuum emphasis is placed on corporate conformance, and at the other the concern is with corporate performance (Bhimani and Soonawalla, 2005).

Given that our research focuses on performance rather than conformance, we need to differentiate between both aspects of corporate governance. We define "corporate governance, conformance perspective" as being related to rules and regulations, and "corporate governance, performance perspective" as referring to performance and value creation.

Working direction of ITG

Most scholars see governance as a top-down phenomenon, commonly based on structure, processes and planning. Another view on ITG is bottom-up. To explain this view we make a side-step to institutional economics, in which two contrasting worldviews coexist, rooted in the $18^{\text {th }}$ century Enlightenment and described as either top-down or bottom-up (Easterly, 2008).

The top-down view of ITG sees the governance of an organisation as being determined by the rules written by the management and leaders of the organisation. In contrast, the bottomup view sees ITG as emerging spontaneously from the social norms, customs, traditions, beliefs 
and values of employees within the organisation, with the governance only formalises what has already been largely shaped by the attitudes of individuals.

Followers of the second perspective often criticise structural, top-down planning processes. For example, Lindblom (1959) has proposed an alternative to the analytical planning approach by "muddling through" with the argument that real world problems are far too complex to solve in such a way. Humans face dilemmas "that are quite different from the wide number of options that management models lay out in front of decision makers" (Ciborra, 1997). Schwarz and Hirschheim (2003) claim that IT executives should approach their governance structure as an "architecture" instead of formalised hierarchies, and argue that researchers "need to change their views of IT 'structure' to embrace a more social and dynamic existence". (Dietz and Hoogervorst, 2012) use the designation distributed governance to clarify that the involvement of the employee should include governance.

While the top-down view is related to structure, processes and planning, the bottom-up view is related to social aspects like culture, behaviour and collaboration.

This results in four ITG streams for the first view (scope) and two ITG streams for the second view (direction). The six streams are summarised in Table 12.

\begin{tabular}{|l|l|}
\hline \multirow{3}{*}{ View } & IT governance stream \\
\cline { 2 - 2 } & 1. IT audit \\
\cline { 2 - 2 } & 2. Decision-making \\
\cline { 2 - 2 } & 3. Part of corporate governance, conformance perspective \\
\cline { 2 - 2 } & 4. Part of corporate governance, performance perspective \\
\hline \multirow{2}{*}{ Direction } & A. Top-down \\
\cline { 2 - 2 } & B. Bottom-up \\
\hline
\end{tabular}

Table 12 Six ITG streams ( Table 1 and Table 12 are equal)

\subsubsection{Results of the Delphistudy}

The participants of the Delphi meeting can be characterised as 14 Dutch CIOs responsible for IT budgets larger than $€ 25$ million. The attendees provided a balanced mix from different branches (see Table 13). 
Section 3

\begin{tabular}{|c|c|}
\hline Attendee \# & Type of organisation \\
\hline 1 & Agriculture \\
\hline 2 & Hospital \\
\hline 3 & Heavy industry \\
\hline 4 & Wholesale \\
\hline 5 & Engineering \\
\hline 6 & Chemical industry \\
\hline 7 & Non-profit \\
\hline 8 & Retail \\
\hline 9 & Power company \\
\hline 10 & Seaport \\
\hline 11 & Health insurer \\
\hline 12 & City \\
\hline 13 & Public health care \\
\hline 14 & Finance \\
\hline
\end{tabular}

Table 13 Meeting attendance

Of the participating CIOs, $57 \%$ report to the $\mathrm{CFO}$ and $21 \%$ to the $\mathrm{CEO}$. Of the remaining three CIOs, one reported to the COO, one was a member of the Board of Directors, and the third had the function of CTO (see Table 14).

\begin{tabular}{|l|c|c|}
\hline \multicolumn{3}{|l|}{ As a CIO I report to: } \\
\hline CEO & 3 & $21 \%$ \\
\hline CFO & 8 & $57 \%$ \\
\hline COO & 1 & $7 \%$ \\
\hline Other & 2 & $14 \%$ \\
\hline Total & 14 & $100 \%$ \\
\hline
\end{tabular}

Table 14 Reporting line

In terms of the question of rating the effectiveness of their current ITG practice (anonymously), $29 \%$ of the CIOs deemed it sufficient (grade $=6 ; 10=$ excellent in the Netherlands). An even larger group of $65 \%$ rated it as good (grade $=7$ or 8 ). Only one CIO rated it as poor (grade $=3$ ) and did not answer the remainder of the questions. 
As regards our second research question, we asked the CIOs to rate the current level of ITG implementation for the first four ITG streams. The answers of the CIOs varied considerably (see Table 15).

\begin{tabular}{|l|c|c|c|c|}
\hline$\#$ & IT auditing & Decision-making & Conformance & Performance \\
\hline Min & $5 \%$ & $10 \%$ & $10 \%$ & $10 \%$ \\
\hline Max & $50 \%$ & $60 \%$ & $50 \%$ & $70 \%$ \\
\hline Mean & $18 \%$ & $33 \%$ & $22 \%$ & $27 \%$ \\
\hline$\sigma$ & $12 \%$ & $17 \%$ & $7 \%$ & $18 \%$ \\
\hline
\end{tabular}

Table 15 Views regarding ITG implementation (regarding scope)

The first two rows show the minimum and maximum values. The third and fourth rows show the arithmetic mean and standard deviation between the answers $(\sigma)$.

Furthermore, we asked participants about the direction of the implementation, i.e. the last two streams.

Five answers were possible: "Completely top-down", "Largely top-down", "Mixed", "Largely bottom-up" or "Completely bottom-up", with a free format field for motivation (see Table 16).

\begin{tabular}{|c|c|c|c|c|c|}
\hline Responses & $\begin{array}{c}\text { Completely } \\
\text { top-down }\end{array}$ & $\begin{array}{c}\text { Largely } \\
\text { top-down }\end{array}$ & Mixed & $\begin{array}{c}\text { Largely } \\
\text { bottom-up }\end{array}$ & $\begin{array}{c}\text { Completely } \\
\text { bottom-up }\end{array}$ \\
\hline$\#$ & 0 & 6 & 5 & 2 & 0 \\
\hline$\%$ & $0 \%$ & $46 \%$ & $38 \%$ & $15 \%$ & $0 \%$ \\
\hline
\end{tabular}

Table 16 Working direction of ITG

As part of the third research question we asked:

"Which disciplines play an important role for ITG in your opinion?"

We added three examples of disciplines (architecture, portfolio management and IT management) to explain what we meant by disciplines. The nonsensical and overly generic answer of "IT management" was added to the examples and accurately detected by each of the CIOs. None of them added this discipline to the list.

The results are summarised in Table 17. The most commonly mentioned disciplines were portfolio management and architecture, which were mentioned by $62 \%$ and $46 \%$ of the CIOs, respectively. 
Section 3

\begin{tabular}{|l|c|c|}
\hline Discipline & Number of times mentioned & $\%$ \\
\hline Portfolio management & 8 & $62 \%$ \\
\hline Architecture & 6 & $46 \%$ \\
\hline Security & 4 & $31 \%$ \\
\hline Project and programme management & 4 & $31 \%$ \\
\hline Demand management & 3 & $23 \%$ \\
\hline Innovation & 3 & $23 \%$ \\
\hline Process design and management & 3 & $23 \%$ \\
\hline
\end{tabular}

Table 17 Important disciplines for ITG according to the CIOs

Thirteen CIOs answered the following question:

"What has to be done to improve ITG, in your opinion?"

Six of them also prioritised the results (see Table 18).

One intention was to cluster the "Actions to improve ITG", but due to a lack of time this was completed afterwards manually by the researchers. The answers are categorised using the six principles of ISO/IEC 38500, which are "Responsibility", "Strategy", "Acquisition", "Performance", "Conformance" and "Human behaviour".

The highest scores were received for "Good ownership of processes and corresponding applications", "Maturity benchmark" and "Educate business managers".

Ownership was seen as especially important by all six CIOs who also prioritised. Owing to duplicate answers this was repeated in "Process owners (having)" and "Responsibility push towards business owners".

Concerning the question "Do you want to be kept informed on the results of the research?" all CIOs responded positively. 
Collect prior research knowledge and assess practical relevance

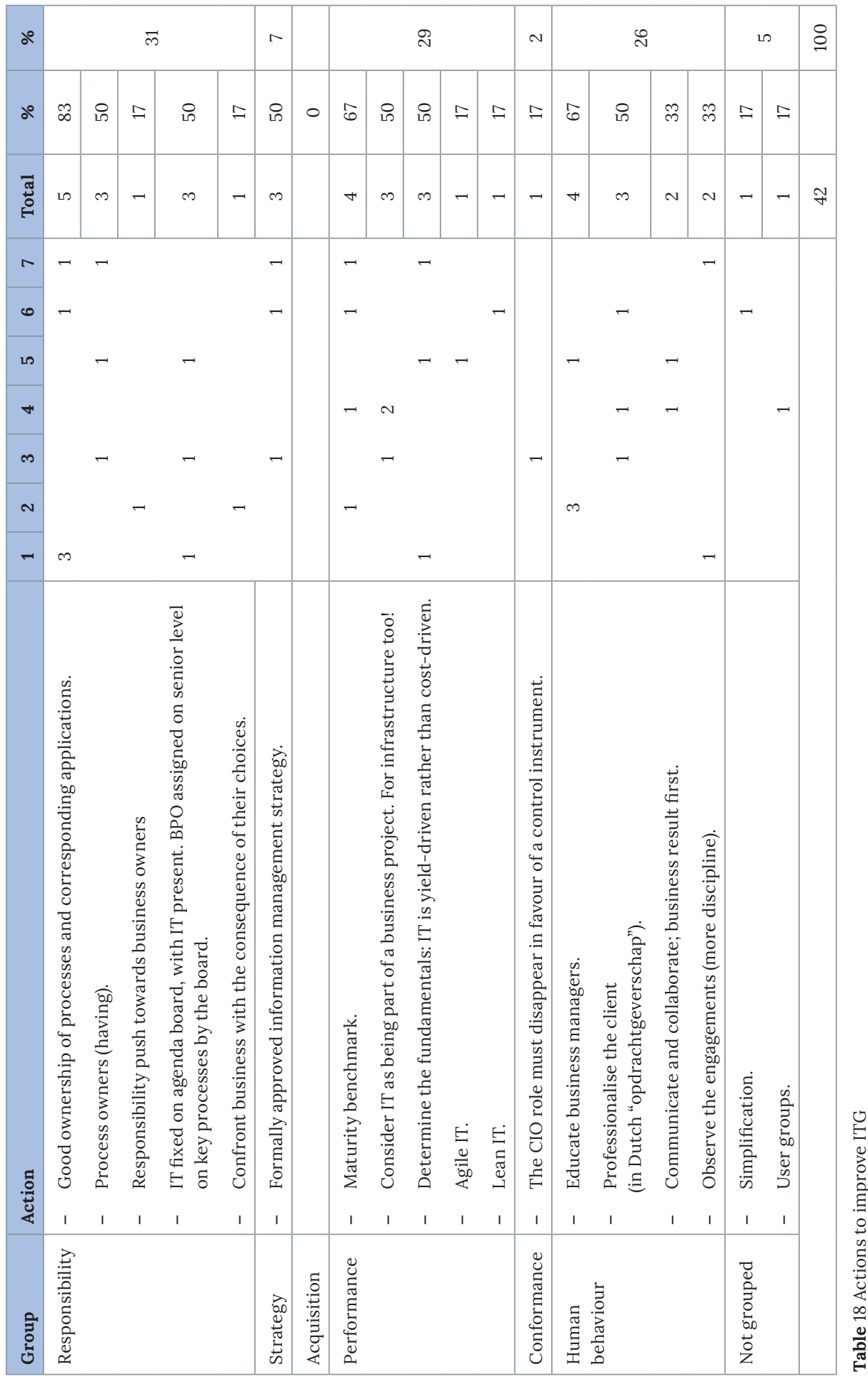


Section 3

\subsection{Discussion}

Current levels of ITG implementation were appraised by the CIOs as sufficient or better (with one exception). This was a surprise. However, it is possible that self-rating results provide a more positive appraisal than an objective evaluation by an independent third party. The discussion concerns our three research questions:

\section{SQ1.1: Which stream(s) of ITG best align with current practices?}

None of the CIOs rated any of the scope streams at $0 \%$, which means that all four streams were considered relevant by the CIOs.

When asked for the working direction of ITG, none of the CIOs chose one of the extremes ("Completely top-down" or "Completely bottom-up"), which means that both were seen as relevant too. Our research further reveals that on the question "What stream is most important?" the CIOs have different opinions.

Therefore, all six ITG streams of the initial model (Table 12) were seen as relevant. Given that the six streams originated in the literature review, the literature and the CIOs agree regarding this aspect. As such, a broad definition of ITG best aligns with current practices.

"Mixed" or "Largely bottom-up" was chosen by $54 \%$ of the CIOs, intimating that more than half of the group deemed bottom-up at least the same or even more important than topdown. Or as one of the CIOs stated, "Bottom-up is naturally just as important" as top-down. Contemporary ITG approaches are mostly top-down.

The fact that none of the CIOs chose completely top-down or completely bottom-up and the relative high score of $54 \%$ for "Mixed" or "Largely bottom-up" implementation relevance can be considered confirmation of the opinions of critics concerning current top-down approaches.

\section{SQ1.2: According to practitioners, which disciplines should play an important role in ITG?}

Our literature review indicated that publications on research covering ITG combined with portfolio management and architecture remain rare and began to grow from 2006/2007. Case studies in literature contain clear descriptions of the ways in which ITG, architecture and portfolio management relate in practice (Wittenburg et al., 2007). Books describing best practices on the combination of disciplines are also available (Niemann, 2006). In the literature, 
architecture and portfolio management are also considered important disciplines for ITG. This was confirmed by the CIOs (see Table 17).

The disciplines perceived as relevant and that were most often mentioned are portfolio management and architecture with $62 \%$ and $46 \%$, respectively. The Delphi study confirmed that the participating CIOs agreed on the relevance of these disciplines and encouraged this direction for our research. The extra disciplines that were advanced by the CIOs presented us with input for additional research.

When considering how to improve ITG, it is interesting to note that the answers to the question "What has to be done to improve ITG?" did not quote disciplines (Table 18). Improvements to portfolio management, architecture, security, project management, programme management or innovation were simply not mentioned. This might be explained by the fact that other issues for improvement besides disciplines with higher priorities existed.

The indications mentioned were of a completely different kind. Most answers (86\%) can be categorised as part of responsibility, performance and human behaviour. The highest score for "Good ownership of processes and corresponding applications" revealed that issues remain regarding responsibilities and accountabilities. As one of the core issues of ITG, this seems a little contrary to the high scores for the rating of the ITG practices. More research is required on this matter.

As a result of our literature review, we found that COBIT is the most commonly used framework in contemporary research papers on ITG maturity. Pertaining to our streams, COBIT especially covers "IT audit" and "top-down". There are critiques of COBIT, especially from the group of scholars we categorized as being in the stream of "bottom-up".

Further research is required to determine why the CIOs do not use existing maturity benchmarks for ITG. The importance of such a benchmark is a welcome confirmation of the relevance of the maturity aspect of our research. It indicates the requirement for another ITG maturity model. This might be the case because contemporary maturity models are largely similar to CMM. CMM is largely based on process maturity, whereas literature and practice indicate the need for maturity models that pay greater attention to the social aspects of ITG.

The high score for "Educate business managers" indicates that there are still some issues in the collaboration between business and IT management. We expect these to be a mixture of content and social factors. 
Section 3

SQ1.4: Is there (still) a mismatch between ITG practice and theoretical frameworks?

The use of internally developed frameworks is diminishing fast, as can be seen in Table 2. In the survey of 2011, this was not an option in the questionnaire. However it was possible to choose the option "Other (please specify)". Given that "Internally developed frameworks" was not included in the tables, we conclude that it tended to decline, with a percentage insufficiently high to reach the results tables (below 1.6\%). Therefore, contemporary organisations do not use internally developed frameworks for ITG.

Compared to the resulting disciplines with the results of the ITGI surveys (Table 2), several aspects attract our attention. Service management (ITIL or ISO/IEC 20000) was ranked top of the ITGI lists, while in our results it was only mentioned twice (Table 17).

Portfolio management, architecture, security and project and programme management were the most frequently mentioned disciplines. PMI/PMBOK and PRINCE2 deal with project, programme and portfolio management, which were also fast growers in the ITGI survey. Architecture has a clear link with TOGAF, which received a negligible percentage of $3 \%$ in the ITGI survey of 2011. Security was in the top four in our research. Several security frameworks like ISO/IEC 17799/27000 are indeed used as the second most frequently used frameworks for ITG in practice (ITGI, 2011).

Thus, we can see that there is substantial correspondence between the mentioned disciplines and the frameworks used in practice. These disciplines however deviate from a conventional list of dimensions, as shown in the example in Table 3.

The literature review also delivered examples of papers in which alternative factors are suggested (Chin et al., 2004, Maidin and Arshad, 2010, Nfuka and Rusu, 2010, Mohamed et al., 2012). Some disciplines can be used to implement some of the dimensions. An example is IT investment management or resource management using portfolio management, and so dimensions, disciplines and frameworks do not match.

Demand management, innovation and process design and management can only be related to the more general frameworks.

The interest of the CIOs in the research is a confirmation of the relevance of our research. 


\subsection{Conclusions}

The research goals of the study were to determine if there remains a mismatch between ITG practices and theory and to collect indications on how to improve ITG using the literature and experts from practice. To answer these questions we defined three sub-questions. The conclusions are grouped around these questions.

SQ1.1: Which stream(s) of ITG best align with current practices?

We defined six ITG streams, four of which define the scope of ITG. ITG can be seen as an audit process, as IT decision-making, as an integral part of corporate governance from a conformance perspective and as an integral part of corporate governance from a performance perspective. Two streams define the direction in which ITG works (top-down or bottom-up).

Our results show that ITG is a broad working field in which all six streams (as shown in Table 12) are considered relevant. "Mixed" or "Largely bottom-up" were chosen by $54 \%$ of the CIOs, and hence more than half of the group considered bottom-up as at least as or even more important than top-down. Contemporary ITG approaches are mostly top-down.

We conclude that a broad definition of ITG best aligns with current practices and that these six streams represent an effective way of studying the diversity of ITG practices. There was consensus between the CIOs in the Delphi study on the relevance of each stream in the initial model and thus on the initial model itself (Table 12).

SQ1.2: $\quad$ According to practitioners, which disciplines should play an important role in ITG?

In the literature, constructs such as dimensions (as shown in Table 3), focus areas (as in COBIT) or principles (as in ISO/IEC 38500) are often used. For recognition in practice we use the more practical term "discipline". The most commonly mentioned disciplines were portfolio management and architecture, being identified by $62 \%$ and $46 \%$ of the CIOs, respectively (Table 17). Publications on research covering ITG combined with portfolio management and architecture remain rare but seemed to start growing from 2006/2007.

When asked on what should be done to improve ITG, the disciplines did not show up again. The highest scores for indications to improve ITG were "Good ownership of processes and corresponding applications", a "Maturity benchmark" and "Educate business managers". 
Section 3

Thus, current ITG maturity models seem to deliver no or scarcely appropriate maturity benchmark information. This indicates the need for another ITG maturity model.

\section{SQ1.3: Is there (still) a mismatch between ITG practice and theoretical frameworks?}

We conclude that there is substantial correspondence between the mentioned disciplines and the frameworks used in practice. These disciplines however deviate from conventional lists of dimensions, e.g. Table 3.

There is no clear congruence between the disciplines and the frameworks used in practice. Given that dimensions, disciplines and frameworks do not match, we conclude that a mismatch between ITG practice, frameworks and theory is also likely.

\section{Implications of the research}

Some scholars prefer a small scope for ITG. Our study shows that a broad definition of ITG best aligns with current practices. One of the reasons why ITG continues to be a "top 10" CIO management issue might be an oversimplification of the reality in contemporary organisations. Current approaches and frameworks are mostly "top-down", based on process, structure and planning. This thesis shows that "bottom-up" is just as important. This means that frameworks that lack sufficient attention to the social aspects of ITG are incomplete.

We conclude that there is a need for a framework and/or an ITG maturity model that combines elements such as process, structure and planning, as can be found in current frameworks with elements pertaining to social aspects like behaviour, collaboration and culture.

\section{Limitations of the research}

The data collection was limited to a small number of Dutch CIOs, and the results might be affected by the culture of Dutch organisations. Thus, the composition of the group and the country of origin may have influenced the results.

It would therefore be interesting to find whether the six streams considered relevant by CIOs are the same in other countries. Further research should be undertaken to determine the implications of the indications to improve ITG collected in the literature review and the Delphi study. 


\subsection{Summary of this section}

This section has provided a detailed description of a systematic ITG literature review and a Delphi workshop with CIOs conducted in 2012 and 2013. The literature review resulted in six ITG streams (see Table 12), all of which were considered relevant by the CIOs in the Delphi study. This indicates that a broad definition of ITG best aligns with current practices.

Given that dimensions, disciplines and frameworks do not match, we consider it likely that a mismatch between ITG practice, frameworks and theory continues to exist. Our initial proposition of the relevance of architecture and portfolio management was confirmed by the CIOs, but as will be shown in Section 5, this did not reappear in the Delphi workshops when determining the focus areas in the design process of the ITG maturity model.

The design of a framework and an ITG maturity model combining "top-down" elements like processes and structure with more social "bottom-up" elements such as behaviour, collaboration and culture will form the next step in our research. In addition, we will determine the focus areas and capabilities required to improve ITG.

As five years have passed since the initial literature study, this investigation was repeated in 2017 / 2018. The following section describes the results of the update of the ITG literature review. 


\section{Section 4}

\section{Systematic literature review on ITG (2018 update)}

\subsection{Introduction}

In 2013 and 2014, as part of the first part of Step II "Collect prior knowledge and assess practical relevance", we published the results of a systematic ITG literature review. In 2018 we conducted an update of the systematic ITG literature review.

The activities and results discussed in this section are marked in light blue in Figure 9.

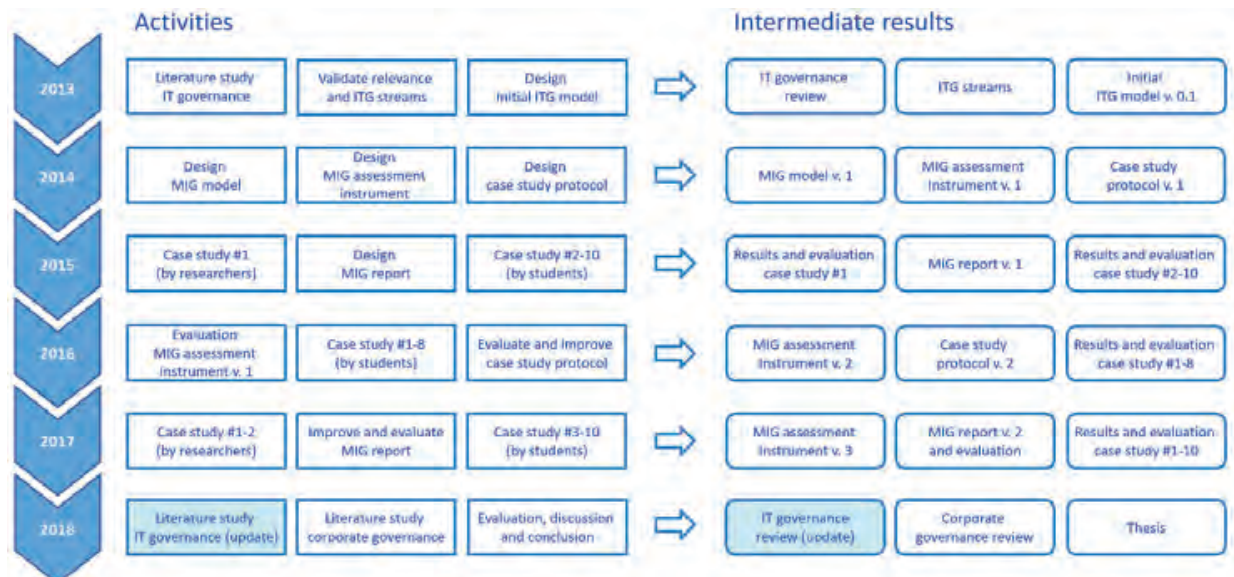

Figure 9 Activities of Step II, Part B.

The research methods for these activities has been described in Section 2.2. The details of this specific study and the results are described in this section.

When updating the systematic ITG literature review we only evaluated new papers. The purpose of the update was to check if the conclusion of our earlier studies remained valid, and to collect an up-to-date overview of available ITG maturity models.

The content of this section is based on earlier published work. ${ }^{5}$

5 Smits, D. \& van Hillegersberg, J. 2018a. The continuing mismatch between IT governance maturity theory and practice: a new approach. International Conference on ENTERprise Information Systems (CENTERIS), Lisbon, Portugal. Elsevier, pp 549-560. 


\subsection{Systematic literate study on ITG: 2018 update}

Previous research indicated a mismatch between the ITG literature and practice (Smits and van Hillegersberg, 2013, 2014a, ITGI, 2011).

These studies are based on surveys and systematic literature reviews using abstract and citation databases until spring 2013. New ITG maturity research covering this gap might have been published. This review was intended to determine if new ITG maturity models have become available recently.

The purpose of the 2018 update was to answer the following two research questions:

SQ1.3: Which (new) ITG maturity models are available in the literature that can be used in practice?

SQ1.4: Is there (still) a mismatch between ITG practice and theoretical frameworks?

\subsubsection{Research method}

In order to create an overview of new ITG maturity models, we conducted a systematic literature review (Petticrew and Roberts, 2006) using Scopus. Scopus is the world's largest abstract and citation database and includes scholarly journals and book publishers. To avoid missing relevant papers, we added some overlap and selected papers from 2012 until the present day (spring 2018). In Scopus, we first selected papers related to "IT governance", "governance of IT", "IS governance" or "enterprise governance" in the title, abstract, or author keywords. Within this large set of papers, we selected papers related to "mature" or "maturity".

A manual selection was used thereafter to determine which papers were in scope. To be included in scope, the paper had to satisfy the following rules:
a. The topic of the paper must be ITG;
b. The keyword "mature" or "maturity" must be used relating to ITG;
c. The publication year must be 2012 or later;
d. The paper must be written in English, German or Dutch;
e. Claims must be justified or based on research;
f. Duplicate studies were excluded.

\subsubsection{Results of I TG}

The first and second selection resulted in a set of 659 documents (excluding duplicates, see Table 9). The update was conducted between January and April 2018 and resulted in an additional set of 471 documents (see Table 19). 
Systematic literature review on ITG (2018 update)

\begin{tabular}{|l|c|c|}
\hline Documents & $\#$ & \% \\
\hline $1^{\text {st }}$ and $2^{\text {nd }}$ selection $(8 / 2012-5 / 2013)$ & 659 & + \\
New papers found $(1 / 2018-4 / 2018)$ & 471 & - \\
\hline Total selected & 1,130 & \\
Removed duplicates or no-papers & 36 & 100 \\
\hline Total excluding duplicates & 1,094 & 30.3 \\
\hline In scope (1'st and 2nd selection) & 331 & 22.4 \\
\hline In scope (new papers) & 245 & $\mathbf{5 2 . 7}$ \\
\hline Total in scope & $\mathbf{5 7 6}$ & \\
\hline
\end{tabular}

Table 19 Totals systematic literature review

After removing duplicates and other types of documents (no research papers), a set of 1,094 documents remained. Having applied the other selection criteria and removing the papers previously found, a set of 245 new papers remained.

The complete set

The oldest documents in our complete set of 576 documents came from 1995 but the vast majority were published from 2006 (see Table 20).

\begin{tabular}{|c|c|c|c|c|}
\hline Year & $1^{\text {st }}$ and $2^{\text {nd }}$ & new & $\sum \#$ & $\%$ \\
\hline 1995-2002 & 12 & & 12 & 2 \\
\hline 2003 & 4 & & 4 & 1 \\
\hline 2004 & 5 & & 6 & 1 \\
\hline 2005 & 11 & 1 & 12 & 2 \\
\hline 2006 & 24 & 2 & 26 & 5 \\
\hline 2007 & 21 & & 21 & 4 \\
\hline 2008 & 35 & 4 & 39 & 7 \\
\hline 2009 & 41 & 5 & 46 & 8 \\
\hline 2010 & 55 & 5 & 60 & 10 \\
\hline 2011 & 67 & 1 & 68 & 12 \\
\hline 2012 & 50 & 10 & 60 & 10 \\
\hline 2013 & 5 & 45 & 50 & 9 \\
\hline 2014 & & 59 & 59 & 10 \\
\hline 2015 & & 33 & 33 & 6 \\
\hline 2016 & & 35 & 35 & 6 \\
\hline 2017 & & 33 & 33 & 6 \\
\hline
\end{tabular}




\begin{tabular}{|c|c|c|c|c|}
\hline Year & $\mathbf{1}^{\text {st }}$ and $2^{\text {nd }}$ & new & $\sum \#$ & \% \\
\hline 2018 & & 12 & 12 & 2 \\
\hline Total & 331 & 245 & 576 & 100 \\
\hline
\end{tabular}

Table 20 Year of publication of the documents

Documents from 2017 and 2018 were limited owing to the time of the selection and the fact that it always takes some time before publications are added to the databases.

\subsubsection{Results of ITG maturity}

The update of the systematic literature review was performed between January and April 2018. This resulted in an initial list of 70 new papers discussing ITG maturity between 2012 and 2018.

After implementing the selection criteria, 34 papers remained.

For each paper, we determined which framework or model was used. The results of this analysis are summarised in Table 21 and Table 22.

\begin{tabular}{|c|c|c|c|}
\hline \# & Model/framework found & \# of papers & List of the papers found \\
\hline 1 & COBIT $4.0 ; 4.1$ and 5.0. & 13 & $\begin{array}{l}\text { (Ateşer and Tanriöver, 2014, Ibrahim and Nurpulaela, } \\
\text { 2016, Ishaq et al., 2017, Janahi et al., 2015, Joshi et al., } \\
\text { 2018, Kosasi, 2015, Putri et al., 2017, Safari and Jiang, } \\
\text { 2018, Seyal et al., 2016, Surya and Surendro, 2014, } \\
\text { Tambotoh and LATUPERISSA, 2015, Vugec et al., } \\
\text { 2017, Spremić, 2012) }\end{array}$ \\
\hline 2 & $\begin{array}{l}\text { COBIT } 4.1 \text { or } 5.0 \text { combined } \\
\text { with other frameworks. }\end{array}$ & 5 & $\begin{array}{l}\text { (Dalipi and Shej, 2012, Ngoma and Erasmus, 2017, } \\
\text { Ningsih et al., 2013, Wahab and Arief, 2015, Wijayanti } \\
\text { et al., 2017) }\end{array}$ \\
\hline 3 & M2A3-ITG model. & 2 & (de Moraes, 2013, de Moraes, 2014) \\
\hline 4 & Nine ITG categories. & 2 & (Shaw et al., 2013a, Shaw et al., 2013b) \\
\hline 5 & Green ITG model. & 1 & (Putri and Muljoredjo, 2014) \\
\hline 6 & $\begin{array}{l}\text { ITG and operation } \\
\text { framework. }\end{array}$ & 1 & (Zhu and Li, 2014) \\
\hline 7 & $\begin{array}{l}\text { Other types of maturity } \\
\text { related research. }\end{array}$ & 6 & $\begin{array}{l}\text { (Alagha, 2013, Albayrak and Gadatsch, 2012, Bianchi } \\
\text { and Sousa, 2015, Elagha, 2014, Saetang and Haider, } \\
\text { 2012, Yaokumah et al., 2015) }\end{array}$ \\
\hline \multirow[t]{2}{*}{8} & MIG model. & 4 & $\begin{array}{l}\text { (Smits and van Hillegersberg, 2013, Smits and van } \\
\text { Hillegersberg, 2014b, Smits and van Hillegersberg, } \\
\text { 2015, Smits and van Hillegersberg, 2017) }\end{array}$ \\
\hline & Total & 34 & Papers \\
\hline
\end{tabular}

Table 21 New papers describing ITG maturity-related research.

The origin of the maturity model, the primary topic and the type of the maturity model of each maturity model are summarised in Table 22. 
Systematic literature review on ITG (2018 update)

\begin{tabular}{|c|c|c|c|c|}
\hline \# & Model/framework found & Origin & Primary topic & Type \\
\hline 1 & COBIT $4.0 ; 4.1$ and 5.0. & $\begin{array}{l}\text { Research \& } \\
\text { practice }\end{array}$ & ITG & Derived from CMM \\
\hline 2 & $\begin{array}{l}\text { COBIT } 4.1 \text { or } 5.0 \text { combined with } \\
\text { other frameworks. }\end{array}$ & - (Diverse) & ITG & - \\
\hline 3 & M2A3-ITG model. & Research & $\begin{array}{l}\text { Model of } \\
\text { Action of ITG }\end{array}$ & $\begin{array}{l}\text { Three specific levels of } \\
\text { maturity }\end{array}$ \\
\hline 4 & Nine ITG categories. & Research & $\begin{array}{l}\text { ITG } \\
\text { performance }\end{array}$ & $\begin{array}{l}\text { A scale of } 20-100 \text { for } \\
\text { governance performance }\end{array}$ \\
\hline 5 & Green ITG model. & $\begin{array}{l}\text { Research \& } \\
\text { practice }\end{array}$ & Green ITG & Derived from ITCMF \\
\hline 6 & ITG and operation framework. & Research & $\begin{array}{l}\text { IT } \\
\text { transformation }\end{array}$ & Five specific levels of maturity \\
\hline 7 & The MIG model. & $\begin{array}{l}\text { Research \& } \\
\text { practice }\end{array}$ & ITG & Focus Area Maturity Model \\
\hline 8 & $\begin{array}{l}\text { Other types of maturity-related } \\
\text { research. }\end{array}$ & $\begin{array}{l}\text { No maturity } \\
\text { model }\end{array}$ & - (divers) & - \\
\hline
\end{tabular}

Table 22 Characteristics of the ITG maturity models found.

Origin: $\quad$ Origin of the model: practice or research.

Primary topic: $\quad$ Primary topic of the maturity model.

Type: $\quad$ Type of the maturity model.

As shown in earlier reviews, COBIT was used in the largest proportion of papers (13) and in five additional papers was combined with other frameworks. In the literature review we found five (relatively) new ITG maturity models partly based on previous research:

- $\quad$ M2A3-ITG model;

- $\quad$ Nine ITG categories;

- $\quad$ Green ITG model;

- $\quad$ ITG and operation framework;

- $\quad$ MIG model.

\subsection{Discussion}

In this section we will discuss each of the eight groups of maturity-related papers:

1. COBIT

The largest part of the set is based on COBIT (13) or COBIT combined with other frameworks (5). ISACA first released COBIT in 1996. There have been several iterations of the COBIT framework to the current version of COBIT 5. COBIT has transitioned from 
an IT auditing framework towards a broader IT governance and management framework with management tools including metrics, critical success factors, maturity models, and tools. Most papers are based on COBIT 4.1 (14 out of 18). Although COBIT version 5 has been published, COBIT 4.1 remains in widespread use in most organisations (Ateşer and Tanriöver, 2014). Some authors use this as a motivation to select version 4.1 (Ishaq et al., 2017), whereas others do not make an explicit distinction and use the version implemented by a corporation (Vugec et al., 2017).

In 2008 and 2009 COBIT was complemented with the Val IT and Risk IT frameworks (ISACA, 2009, ISACA, 2008). COBIT 5.0 was released early in 2012 to cover all aspects that stimulate effective ITG, such as "culture, and so on over and above processes" (Oliver and Lainhart, 2012) by defining "a holistic approach" as a driving principle (ISACA, 2012). The differences between version 4 and 5 are significant and the result of several important developments influenced by changes in the external environment as well as new and revised frameworks to which COBIT aligns. Besides other changes, COBIT 5.0 now includes a separation between governance and management, integrates the best practices of COBIT 4, Val IT, and Risk IT, and has an improved assessment of process maturity, a core metric in COBIT, and is aligned with international standards (De Haes et al., 2013). The new governance domain in COBIT 5.0 has five processes that would be in the hands of the board and the most senior management.

COBIT made use of the Capability Maturity Model (CMM) process until version 4.1 and computed as the weighted mean of the respondents' distribution on the six possible maturity levels starting from non-existent (0) to optimised (5) (ITGI, 2007, Pederiva, 2003). In COBIT 5.0 this was replaced by process capability as defined in the ISO/IEC 15504 standard (ISO/IEC 15504, 2004). The new Process Capability approach results in an improvement of the assessment process, and in particular in the formality and rigour of the assessment (Pasquini and Galiè, 2013).

\section{COBIT combined with other frameworks.}

The research papers using COBIT combined with other frameworks are very diverse. In these papers COBIT was combined with the service management framework: ITIL (Dalipi and Shej, 2012, Ngoma and Erasmus, 2017), the open group architecture framework: TOGAF (Ningsih et al., 2013, Wahab and Arief, 2015), a specific ITG framework used in South Africa: DIPSA (Ngoma and Erasmus, 2017), total quality management: TQM (Dalipi and Shej, 2012) and the business balanced scorecard: BSC (Wijayanti et al., 2017). 
3. M2A3-ITG model

The M2A3-ITG model is a Maturity Model for Analysis of Alignment of Activities related to ITG and presented in De Moraes' thesis (2013). The twelve fields of action are a research model to "assess the degree of effectiveness of IT actions to meet the expectations of the Strategic Plan of the Organization" (de Moraes, 2014). The focus of the M2A3-ITGov Model is the Assessment Maturity Level. The indicators constructed by the model are "direct, relevant and practical result" indicators (de Moraes, 2013). The twelve fields of action of IT are auditing, compliance, development, knowledge, management, planning, production, project, quality, requirement, security and testing. In the M2A3-ITG model, 51 result indicators are defined for these fields of action. The model defines three maturity levels for a result indicator A until C, in which A corresponds with a "Complete match", B with a "Match with restrictions" and C as "No match" with respect to the expectations of the corporation.

4. Nine ITG categories.

The nine ITG categories form a research model to investigate the effects of ITG categories on governance performance (Shaw et al., 2013b), and based on the proposed nine ITG implement categories of Itakura's (2007) ITG organisational capabilities view. The nine ITG categories are: user support, decision-making of top management, review and evaluation IT tasks, ability and evaluation of IT department, risk management, CIO authority, budgeting process, outsourcing and IT project development management. In order to measure the governance performance, Weill and Ross' (2004) formula was used (2004). This formula measures four effects ("cost-effective use of IT", "effective use of IT for growth", "effective use of IT for asset utilisation" and "effective use of IT for business flexibility") on a scale of 1 ("Not important") to 5 ("Very important"). This resulted in a minimum and maximum possible governance performance of 20 and 100, respectively.

\section{Green ITG model}

The green ITG model is a research model for private higher education institutions, developed in the capital region of Jakarta, Indonesia (DKI Jakarta) for use in private higher education institutions to minimise energy consumption (pull) and money (push) (Putri and Muljoredjo, 2014). The push model - focusing on vertical activities - was adopted from material resource planning. It uses calculation and production schedule 
for every level, based on sales forecast. The pull model -focusing on horizontal activitiesoriginated in the just-in-time (JIT) system used in manufacturing. In JIT, production is triggered by customer demand: the users are pulling what they need.

A consortium of leading organisations from industry, the non-profit sector and academia (the Innovation Value Institute) has developed a framework to improve sustainable IT capabilities: the Sustainable ICT-Capability Maturity Framework (SICT-CMF) (Donnellan et al., 2011), based on the IT Capability Maturity Framework (IT-CMF).

The green ITG model is based on SICT-CMF and the four basic sustainable IT postures of Curry et al. (2012): the cost centre, the service centre, the investment centre and the value centre. This results in four horizontal activities:

- IT data centre;

- IT efficiency technique;

- facility efficiency technique;

- integration efficiency technique;

and vertical activities depending on material elements, comprising printing-paperless, reuse-recycle and rules-policy.

6. ITG and operation framework.

Zhu and Li (2014) have designed an ITG framework, operating model and IT maturity model for IT transformation design. For the governance framework, four mechanisms have been identified and integrated: organisation, processes, compliance and transformation. This design was developed based on industry best practices and standards such as COBIT, Val-IT and ITIL. The IT operating model covers IT functional structure, IT operational processes, consistent matching and transformation management.

The IT maturity model was designed based on preliminary findings in a survey involving more than 100 large-scale chemical enterprises between 2008 and 2013. It covers an IT and a business side with five levels each:

1. Technology-driven - customer follows;

2. Controlled - customer chooses;

3. Service-oriented - customer decides;

4. Customer-driven - customer owns;

5. Business-driven - customer directs.

The research paper is rather short (four pages), and so provides minimal details about the design process and the survey. 


\section{Systematic literature review on ITG (2018 update)}

7. The MIG model.

The MIG model is the topic of this thesis and the design of the model described in detail in Section 5.

8. Other types of maturity-related research.

Research papers not specifying or using an ITG maturity model.

An overview:

A theoretical study using data from 20 Emirati organisations to evaluate how a firm's five governance domains affect the level of IT governance maturity and how a firm's five proposed governance mechanisms shape the overall effectiveness of ITG (Alagha, 2013). In this research, Dahlberg and Kivijärvi's (2006) five ITG domains are used:

1. Alignment of business and IT;

2. Monitoring of IT resources, risks and management;

3. Monitoring of IT performance measurement;

4. Evaluation of value delivery;

5. ITG development.

Albayrak and Gadatsch (2012) describe an integrated reference model for IT performance measurement based on a life-cycle model and a performance-oriented framework. The reference model does not include a maturity model.

Bianchi and Sousa (2015) describe the intended design science approach to develop an ITG model with structures, processes and relational mechanisms suitable for public sector universities with guidelines for effective and efficient IT governance.

A short paper - 3 pages - describing a study based on data from 20 organisations within financial services, telecommunications, manufacturing, and public service as identified the most influential ITG domain for increasing the level of ITG maturity (Elagha, 2014). It makes use of partial least squares path modelling and finds monitoring of IT performance measurement to be the most influential ITG domain, and the implementation of a corporate communication systems as the most influential ITG mechanism. Saetang and Haider (2012) have developed a research framework for 
investigating effective ITG implementation using the Duality of Technology (Orlikowski, 1992) and the Adaptive Structuration Theory (DeSanctis and Poole, 1994). This is interesting because they can be considered alternative representations of hard and soft governance. The Duality of Technology model concerns the dualism between objective, structural features of organisations and subjective, knowledgeable action of human agents. In other words: the interplay between the types of structures inherent to technologies and the structures that emerge in human action as people interact with these technologies. DeSanctis and Poole (1994) extend the structuration models of technology-triggered change to consider the mutual influence of technology and social processes. Seating and Haider's 2012' research aims to present the constituent elements in ITG implementation, reflecting a structural, top-down understanding between the organisation, people and IT in developing better internal relationships within an organisation. This appears to influence organisational maturity through the performance evaluation phase. The research framework lacks a maturity component but rather measures organisational maturity through "performance evaluation as the outputs of organizational environment which embrace the performance of human and IT" (Saetang and Haider, 2012). A study using a survey questionnaire to determine the status of ITG in universities in a developing country (Ghana) through assessing the drivers and barriers to pursuing formal ITG has measured the extent to which universities align IT goals with academic and business objectives in order to determine the ITG maturity level (Yaokumah et al., 2015).

\subsection{Conclusion}

This section summarises the answers to the research questions of section 4:

\section{SQ1.3: Which (new) ITG maturity models are available in the literature that can be used in practice?}

The systematic literature review revealed that 34 new papers discussing ITG maturity have been published since 2012. COBIT was used in 13 papers, while five others combined it with other best 
practice frameworks. COBIT 5.0 includes the social dimension by discerning seven enablers: "Principles, policies and frameworks", "Processes", "Organizational structures", "Culture, ethics and behaviour", "Information", "Services, infrastructure and applications" and "People, skills and competences", translating them into four common dimensions: "Stakeholders", "Goals", "Life-cycle" and "Good practices" (Oliver and Lainhart, 2012).

In the literature review we found five (relatively) new ITG maturity models, partly based on previous research:

- $\quad$ M2A3-ITG model;

- $\quad$ Nine ITG categories;

- $\quad$ Green ITG model;

- $\quad$ ITG and operation framework;

- $\quad$ MIG model.

Each maturity model was described in detail in the previous section (see 4.3). The MIG model represents the topic of this thesis.

SQ1.4: Is there (still) a mismatch between ITG practice and theoretical frameworks?

We found two frameworks covering hard and soft ITG: COBIT 5.0 in a holistic way and the MIG model in a more practical way.

We will return to this research question in more detail in Section 9.2, as it is partly based on the results discussed in the following sections of the thesis.

\subsection{Summary of this section}

This section provides a detailed description of the 2018 update of the systematic ITG literature review.

This update was conducted with two purposes:

- $\quad$ To determine if (new) ITG maturity models became available in the literature since the initial systematic literature ITG review in 2013;

- $\quad$ To determine if the conclusion to the research question "Is there (still) a mismatch between IT governance practice and theoretical frameworks?" (SQ1.4) in the earlier review remains valid. 
The update revealed that it still seems likely that there is a mismatch between ITG practice, frameworks and theory. In addition to our MIG model, we found five (relatively) new ITG maturity models. As a result we found two frameworks covering hard and soft ITG: COBIT 5.0 in a holistic way and the MIG model in a more practical way.

The next part of the thesis describes the "Design and evaluation of the MIG model and the MIG assessment instrument".

The topic of the following section is "The design of the MIG model". 


\section{Part I I I}

\section{Design and evaluation of the MIG model and the MIG assessment inst rument}

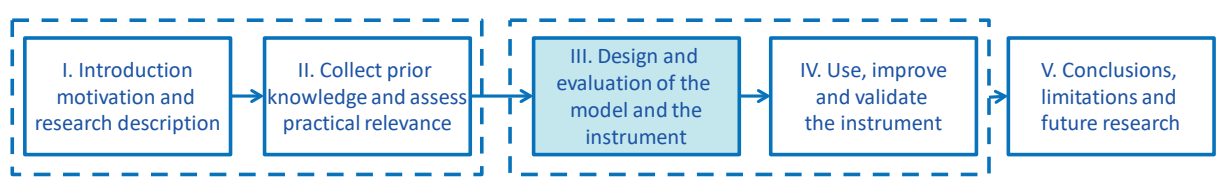

Figure 10 Thesis Part III. Design and evaluation of the model and the instrument

\section{Section}

5. The design of the MIG model

6. The design and evaluation of the MIG assessment instrument 


\section{Section 5}

\section{The Design of the MIG Model}

\subsection{Introduction}

Step III covers the design and evaluation of the MIG model and instrument. An overview of the activities and results discussed in this section is shown in the research outline and marked in light blue in Figure 11.

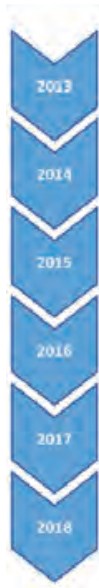

Activities
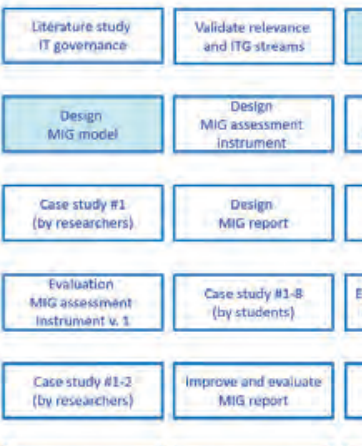

Literature study IT governance (update)
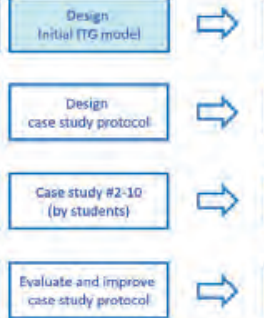
Evaluate and improve
case study protocol
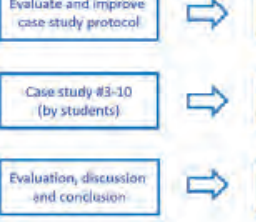

Intermediate results
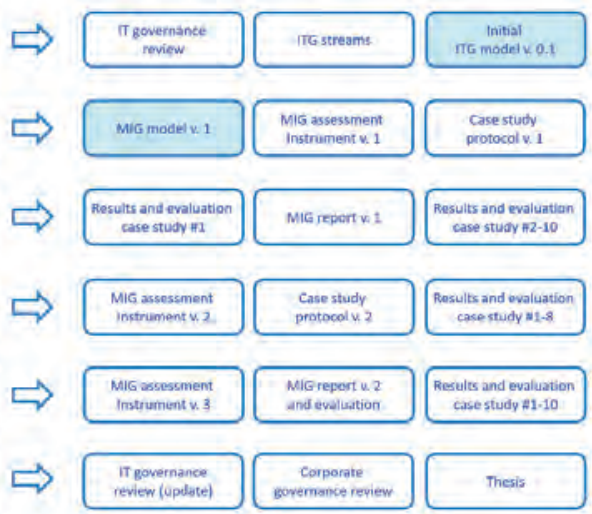

Figure 11 Activities and results of Step III, Part A.

The research method for these activities has been described in Section 2.2. The details of the design process of the MIG model and the results are described in this section.

In this section we describe the results of the following two activities:

- $\quad$ The design of the initial ITG model v. 0.1;

- $\quad$ The design of the MIG model v. 1.

The rationale behind this research was the absence of usable frameworks and models to implement ITG in practice. Maturity models used for ITG are related to the existing frameworks which are largely focused on processes and structure.

One of the challenges in this research is how to combine the hard and soft side of ITG. 
In the years before this research I have gained experience with the use of a focus area maturity model for enterprise architecture (van Steenbergen et al., 2010). That gave rise to the idea to combine the hard and soft side by designing a focus area maturity model for ITG.

There was no intention to create something completely new. We continued our research looking for existing models to capitalize on existing research. A well-known classification comprises the three layers of Peterson, O'Callaghan and Ribbers (2000): structural integration, functional integration and social integration. In 2004 this became better known (and somewhat simplified) as the trichotomy of structure, processes and relational mechanisms (Van Grembergen, 2004). This model was selected because it already includes some soft elements. Furthermore, we used the results of the systematic literature review to select an initial list of focus areas and corresponding maturity models to design the initial version of the MIG model.

Maturity models are a concept widely used and of considerable relevance to IS practice and IS research (Pöppelbuß et al., 2011, Röglinger et al., 2012). The designs of the maturity models "have only been documented very sketchily" (Becker et al., 2009). In this thesis the design process was described in detail. In the design process for the maturity model we combined the guidelines for design science (Hevner et al., 2004, Hevner and Chatterjee, 2010, Gregor and Hevner, 2013) with more specific steps for the design of (focus area) maturity models described in detail in the next Section.

The content of this section is based on earlier published work. The design of the MIG model was published in two separate papers:

- $\quad$ a paper describing the design of the model and the resulting focus areas ${ }^{6}$;

- $\quad$ a paper describing the corresponding maturity models for each focus area?

\subsection{Research method}

The systematic literature review described in Section 3 demonstrated that an ITG maturity model for hard and soft governance does not exist. This has also been illustrated in recent literature reviews and surveys (ITGI, 2011, Pöppelbuß et al., 2011).

6 Smits, D. \& van Hillegersberg, J. 2014b. The development of an IT governance maturity model for hard and soft governance. 8th European Conference on IS Management and Evaluation (ECIME), Brussels, Belgium. Academic Conferences and Publishing International Limited, pp 347-355.

7 Smits, D. \& van Hillegersberg, J. 2015. IT governance maturity: Developing a maturity model using the Delphi method. 48th Hawaii International Conference on System Sciences (HICSS), Kauai, Hawaii, USA. IEEE, pp 4534-4543. 
We consequently designed a new maturity model for ITG using knowledge from the literature and experts. In Section 5 we will discuss the answers to the following three research questions: (see Section 2.3.1)

SQ2.1: Which focus areas should an ITG maturity model for soft and hard governance contain? SQ2.2: What type of maturity model do we need?

SQ2.3: What are the capabilities of each focus area?

Numerous views can be found on how to design a maturity model, and no shared vision exists on which approach should be followed (Mettler and Rohner, 2009, Pöppelbuß and Röglinger, 2011). As a design process for the maturity model we combined the general process steps as described by Maier, Moultrie and Clarkson (2012) for the design of maturity grids with the requirements of Becker et al. (2009) - especially the iterative development - integrating the process steps and requirements with the more specific process steps for the design of focus area maturity frameworks adopted from van Steenbergen et al. (2010).

We began with a literature review on the maturity model design to select the design method for the maturity model. In preparation for the first meeting with practitioners, an initial version of the maturity model was designed based on current literature. This model will be explained in Section 5.3.

The initial model was discussed in a Delphi study with an initial four and later two rounds.

The MIG model was designed via the following steps: (see Figure 12)

a. The design of the initial ITG model using the information collected during the ITG literature review described earlier (see Section 3.3.1). This resulted in version 0.1 of the MIG model;

b. A Delphi study with four rounds was conducted starting with the initial model to reach consensus about the domains and focus areas of the MIG model (see Section 5.4). This resulted in version 0.7 of the MIG model;

c. As a next step during the Delphi studies, we discussed and reached a consensus regarding the supplementation of maturity models from the literature to the focus areas of the MIG model (see Section 5.5). This resulted in version 0.8 of the MIG model;

d. Combining the results of step b (focus areas) and c (maturity models) resulted in version 0.9 of the MIG model;

e. A Delphi study with two rounds was conducted to solve the remaining issues with the MIG model. This resulted in version 1.0, the final version of the MIG model. 


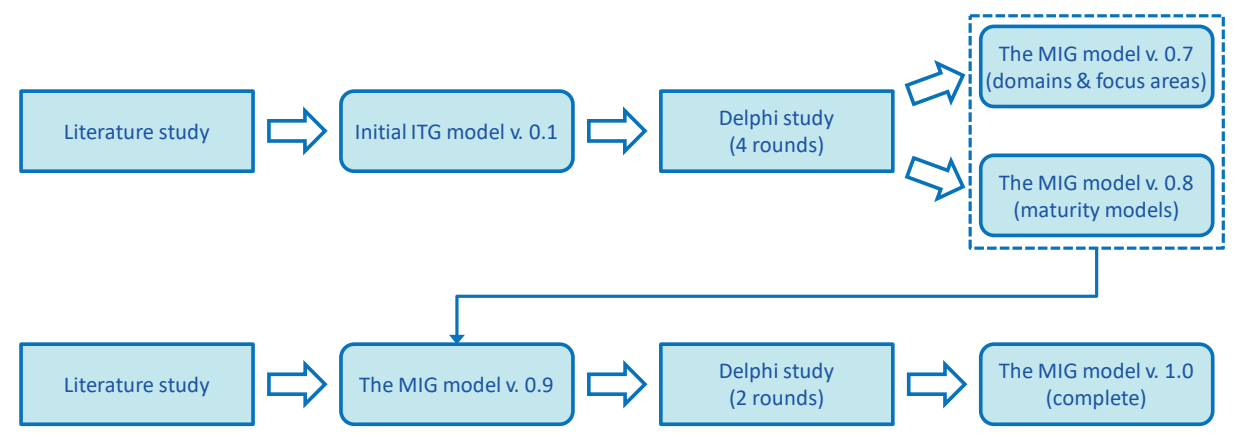

Figure 12 Design process of the MIG model

After each round the model was improved using the feedback from the meetings. The meetings were organised between October 2013 and February 2014, and continued into November 2014 and January 2015. The results of these final two workshops are described in Section 5.6.

It was explained to invitees that it was important to attend the complete series of meetings. When experts invited to the meetings were unable to attend a meeting, they were asked to provide their feedback online at a later time.

Careful selection of participants is important. For the series of meetings, we invited participants with numerous years of experience in ITG. These individuals were found among the members of the special interest group Governance of the Ngi (the Dutch association of IT professionals) and the NAF (Dutch Architecture Forum) working group on IT governance. In this way the research approach combined knowledge from the literature and experts from practice to achieve both "problem relevance" and "research rigour" (Hevner et al., 2004). The resulting MIG model will be validated in organisations in different industry sectors as a next step.

\subsubsection{Technical details of the Delphi study}

For the Delphi study we used the web-based GDSS tool Spilter (see Section 2.2.3).

The participants were required to respond to questions and statements using a laptop or tablet. Figure 13 displays an example of a screenshot of Spilter.

There was no hierarchy or dominance; each opinion counted and could be recorded. Responses were anonymous to the rest of the group. For example, when asked to rate some changes to the model, we used the tool to show graphs of the responses and obtain consensus before proceeding to the next step.

When experts invited to the meetings were unable to attend, they were asked to provide their feedback online (on Spilter) at a later time. Through Spilter, all feedback is traceable to 
the participant. It is thus possible to separate the responses from persons present during the meeting and those participating online.

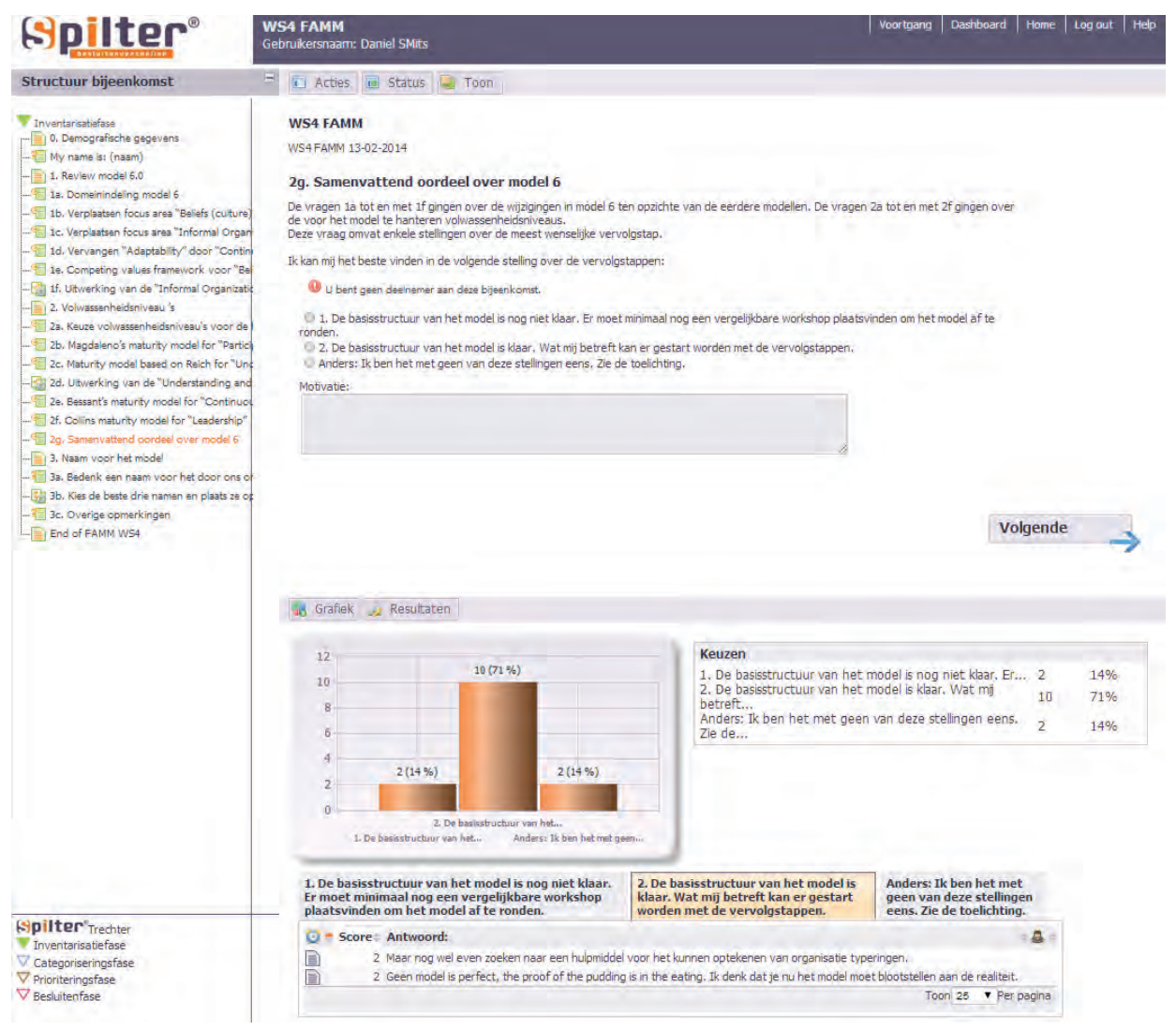

Figure 13 Example screenshot from Spilter

\subsection{The design of the initial model}

The first step in the design of a focus area maturity model is the determination of the ITG domains. We concluded that a broad definition of ITG best aligns with current practices (see Section 3.6) using the broad scope based on six streams. The second step is the selection of a set of focus areas for each domain. The ITG domains could be defined in different ways depending on the definition of ITG adopted.

We define the hard side of ITG as the functional aspects of governance, such as structures and processes. These aspects are also defined as the elements of organisational design. The 
soft side of ITG is defined as relating to social aspects like human behaviour and organisational culture.

The design of the soft side of the initial model required the most attention. The distinction between hard and soft governance has been made before. Moos (2009), for example, differentiates between legislation and the "softer" forms of governance based on persuasion and advice or obligation, precision and delegation (Tucker, 2003). Related to participatory governance, Cook (2010a) deems "rules and structures" as being "far less effective" than soft governance.

Uehara (2010) and Tarmidi et al. (2012) separate hard and soft ITG using the soft power theory. Joseph Nye (1990) founded the soft power theory, arguing that it is related to "intangible power resources such as culture, ideology, and institutions". This is close to how we see soft governance.

In preparation for the first meeting, the usability of several ITG models from the literature and practice such as COBIT, ISO/IEC 38500 (ISO/IEC 38500, 2008) and the ITG trichotomy were discussed with a small group of specialists. As proposed by several scholars, ITG can be deployed using a trichotomy summarised as structures, processes and relational mechanisms. $\mathrm{Be}$ it in the public or private sector, IT governance can be deployed using a combination of processes, structures and relational mechanisms (Ali and Green, 2007, Campbell et al., 2010).

To delineate the necessary elements of our IT governance framework, Peterson (2004), Van Grembergen et al. (2004) and Weill and Woodham (2002) propose that IT governance can be deployed via a combination of structures, processes and relational mechanisms (Wu, 2015). We adopt this model as the starting point for the initial model.

A more detailed analysis of the literature describing this trichotomy highlighted the lack of a clear definition of the ITG domains and some differences in the precise formulation (see Table 23).

\begin{tabular}{|l|l|l|l|}
\hline Domain 1 & Domain 2 & Domain 3 & Source \\
\hline $\begin{array}{l}\text { Structural integration } \\
\text { mechanisms }\end{array}$ & $\begin{array}{l}\text { Functional integration } \\
\text { mechanisms }\end{array}$ & $\begin{array}{l}\text { Social integration } \\
\text { mechanisms }\end{array}$ & (Peterson et al., 2000) \\
\hline $\begin{array}{l}\text { Structural } \\
\text { coordination }\end{array}$ & $\begin{array}{l}\text { Functional } \\
\text { coordination }\end{array}$ & Social coordination & (Peterson, 2001) \\
\hline Structure & Process & Mechanisms & (Weill and Woodham, 2002) \\
\hline Structure & Process & Relational mechanism & (Van Grembergen et al., 2004) \\
\hline
\end{tabular}

Table 23 Evolution of the trichotomy of ITG domains 
The third column in the table indicates that some authors chose to replace the word "social" with the much more restricted phrase "relational". Relational mechanisms or relations between people are relevant for soft governance. However, aspects like culture, values or personal characteristics are also of interest. By incorporating "relational mechanisms" into our model we expected that the collaboration between people would be covered effectively.

To cover the remaining social aspects, we added a fourth domain for the behavioural or social aspects of governance. This domain was named "Behaviour and culture". The significance of behaviour for ITG could be seen in its inclusion as one of the six principles of the ISO/IEC 38500 standard for ITG (ISO/IEC 38500, 2008).

The same sources were used to divide the domains into focus areas. During the elaboration of the model we selected the focus areas from the sources listed in Table 23 that fall within the rather broad concept of human behaviour. These subfields were required for the design of the maturity model.

The descriptions in Peterson's articles were especially useful for this purpose. The focus areas that could be categorised as part of soft governance were moved to the fourth domain "Behaviour and culture". The result of this process was the initial model (Table 24).

\begin{tabular}{|c|c|c|}
\hline Governance & Domain & Focus area \\
\hline \multirow[t]{5}{*}{ Soft } & \multirow[t]{3}{*}{ Behaviour and culture } & Beliefs (values, norms) \\
\hline & & Informal organisation \\
\hline & & Leadership \\
\hline & \multirow[t]{2}{*}{ Relational mechanism } & Participation \\
\hline & & Understanding \\
\hline \multirow[t]{4}{*}{ Hard } & \multirow[t]{2}{*}{ Process } & IT decision-making \\
\hline & & Monitoring \\
\hline & \multirow[t]{2}{*}{ Structure } & Functions and roles \\
\hline & & Formal networks \\
\hline
\end{tabular}

Table 24 The initial ITG model v. 0.1

The initial model consisted of four domains and nine focus areas. For each domain or focus area, a definition was selected based on the sources in Table 23, using the literature to help. 


\subsection{The design of the MIG model}

The design of the MIG model was the result of a Delphi study with four rounds. In this section we summarise the results of these workshops. The number of participants at the first meeting was 19. Participation at the following meetings was only possible for this group of 19 (see Table 25).

\begin{tabular}{|l|c|c|c|c|}
\hline Participation & WS1 & WS2 & WS3 & WS4 \\
\hline On location & 18 & 11 & 10 & 7 \\
\hline Online (afterwards) & 1 & 5 & 4 & 7 \\
\hline Total & 19 & 16 & 14 & 14 \\
\hline
\end{tabular}

Table 25 Number of participants for each meeting (WS: workshop number)

In spite of the request to attend all meetings, some participants were unable to attend the complete series of meetings. The reasons stated were: too difficult (1x); it takes too much time (1x); time and private circumstances (2x); and job loss (1x).

We originally planned three meetings. A fourth meeting was later added in consultation with the participants. The results of workshops five and six are described in Section 5.6. These workshops were planned at a later stage.

The average age of the participants was 51 years. The group consisted of three women and 16 men. Eleven had a Master's degree, six a Bachelor's degree and two followed another type of education ('O'). A specific ITG training was attended by eleven participants (see Table 26).

\begin{tabular}{|c|c|c|c|c|c|c|c|c|}
\hline 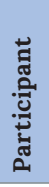 & 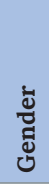 & 足 & 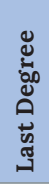 & Current position & 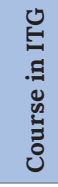 & 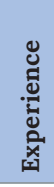 & 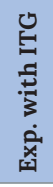 & 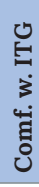 \\
\hline 1 & M & 53 & M & Information Manager & Yes & 25 & 15 & 5 \\
\hline 2 & M & 24 & M & Consultant Information Policy & No & 1 & 1 & 5 \\
\hline 3 & $\mathrm{~F}$ & 49 & M & Consultant & Yes & 14 & 14 & 7 \\
\hline 4 & M & 59 & M & Management Consultant & Yes & 34 & 26 & 7 \\
\hline 5 & M & 59 & B & Audit Manager & Yes & 32 & 29 & 5 \\
\hline 6 & $\mathrm{~F}$ & 50 & B & Operational Auditor & No & 31 & 11 & 2 \\
\hline 7 & M & 59 & B & Interim Manager & No & 35 & 10 & 4 \\
\hline 8 & M & 40 & $\mathrm{O}$ & ICT Architect / Consultant & No & 14 & 3 & 1 \\
\hline 9 & $\mathrm{M}$ & 55 & B & Interim Manager & No & 30 & 3 & 2 \\
\hline
\end{tabular}




\begin{tabular}{|c|c|c|c|c|c|c|c|c|}
\hline 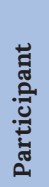 & tே & $\stackrel{\infty}{4}$ & 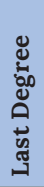 & Current position & 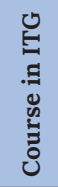 & 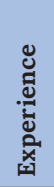 & 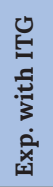 & 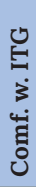 \\
\hline 10 & M & 55 & $\mathrm{O}$ & Enterprise Architect & Yes & 25 & 10 & 6 \\
\hline 11 & M & 58 & M & Strategic Adviser & Yes & 35 & 10 & 5 \\
\hline 12 & M & 44 & B & Management Consultant & Yes & 20 & 18 & 6 \\
\hline 13 & M & 55 & M & Director & No & 29 & 16 & 6 \\
\hline 14 & M & 57 & M & Information Manager CIO Office & No & 30 & 10 & 5 \\
\hline 15 & $\mathrm{~F}$ & 54 & M & Audit Manager & Yes & 26 & 26 & 5 \\
\hline 16 & M & 26 & M & Consultant & Yes & 3 & 3 & 6 \\
\hline 17 & M & 52 & B & Adviser IT Governance (CIO position) & No & 30 & 25 & 7 \\
\hline 18 & M & 61 & M & Consultant & Yes & 25 & 9 & 6 \\
\hline 19 & M & 54 & M & IT manager & Yes & 29 & 6 & 7 \\
\hline
\end{tabular}

Table 26 Participant demographics WS1 - WS4

The average years of work experience was 25, of which 13 years were spent with ITG. Eight participants were consultants and nine were managers or directors. All participants had specific interest in and experience with ITG. The self-reported average expertise with ITG was high: six on a scale of one to seven.

The hard and soft governance aspect of the maturity model uses the capabilities described in the sources listed.

However, the context was considered value-free. The maturity model does not contain capabilities for these areas. The context was used to assess the situational aspects. For this part of the model, frameworks (instead of maturity models) were selected to be utilised as conceptual structures that enabled us to collect, collate and analyse the data for these focus areas.

The changes to the initial model during the meetings of the Delphi study are summarised in Table 27. 


\begin{tabular}{|l|l|l|}
\hline No. & Description of the change & No. workshop \\
\hline 1. & Domain "Relational mechanisms" was changed to "Collaboration" & WS1 \\
\hline 2. & Focus area "Planning" (including "bottom-up") was added to the model & WS1 \\
\hline 3. & Focus area "Continuous improvement" was added to the model & WS1; WS2; WS3; WS4 \\
\hline 4. & Focus area "Understanding" was changed to "Understanding and trust" & WS2 \\
\hline 5. & "Context" was added to the model & WS3 \\
\hline 6. & Focus area "Beliefs (culture)" was moved to "(Internal) context" & WS3 \\
\hline 7. & Focus area "Informal organisation" was moved to "(Internal) context" & WS4 \\
\hline
\end{tabular}

Table 27 The changes to the model (in chronological order)

In the column "When", the moment when the remarks or discussion with the participants occurred is stated. The third change was to a focus area that was altered on several occasions. Only the final result is stated. Each change will be discussed in more detail later in this section.

Following each meeting, an intermediate research model was created based on the results. At the beginning of each meeting the participants were asked to state their opinion on the current model. The progress of the support for the intermediate and final models by the participants during the meetings is shown in Table 28.

\begin{tabular}{|c|c|c|c|c|c|c|c|}
\hline Meeting & Research model & 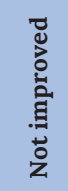 & 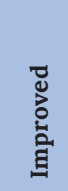 & 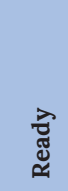 & 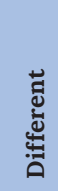 & సี & 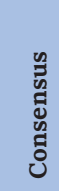 \\
\hline WS2 & Domains interim model & $12 \%$ & $31 \%$ & $56 \%$ & $0 \%$ & $100 \%$ & $87 \%$ \\
\hline WS2 & Focus areas interim model & $6 \%$ & $56 \%$ & $38 \%$ & $0 \%$ & $100 \%$ & $94 \%$ \\
\hline WS3 & Focus areas interim model & $7 \%$ & $36 \%$ & $50 \%$ & $7 \%$ & $100 \%$ & $86 \%$ \\
\hline WS4 & Resulting MIG model & $14 \%$ & $\mathrm{~N} / \mathrm{A}$ & $71 \%$ & $14 \%$ & $100 \%$ & $71 \%$ \\
\hline
\end{tabular}

Table 28 Support for the interim models and the resulting model

In order to implement a change, we determined that an $80 \%$ or greater consensus level was necessary. In a group there is usually some disagreement and participants who have particular preferences cannot be allowed to hamper the results of the group.

The options from which the participants could choose were specific but can be summarised as: the model is "Not improved", "Improved", "Ready" or "Different" opinion. The sum of "Improved" and "Ready" is included in the column "Consensus". During WS4, the choice "Improved" was not available. 
The first discussion concerned the domain "Relational mechanisms". The name "Relational mechanisms" was assessed by the participants as being unclear. During the meeting, "Collaboration" was suggested as an alternative. The replacement of "Relational mechanisms" with "Collaboration" was considered an improvement by $88 \%$ of the participants.

The second change was the addition of the focus area "Planning" to the model. It was added following a discussion on the need for business strategy in the model. When asked to rate the change during WS2, 94\% of the participants stated that it was an improvement. One participant remarked that it is hardly possible to plan in our complex world and preferred to drop it.

The third change was the most discussed during the meetings. The name of this focus area was changed several times from "Changeability" to "Adaptability" and finally to "Continuous improvement". This focus area was always intended to be a characteristic for the organisation. The last change was made based on the notion that learning is required for change in the right direction.

When asked whether "Adaptability" should be replaced by "Continuous learning", 43\% supported the change or did not have a preference (14\%). However, 29\% preferred "Adaptability" and $14 \%$ had other concerns.

The focus area "Understanding" was changed to "Understanding and trust". The reason for this change was a discussion during WS2 on the relevance of trust. This change is also in line with literature. Nelson and Cooprider (1996) have highlighted that shared knowledge and trust leads to increased performance in an IT department. Through this shared knowledge base, barriers to understanding and acceptance between IT departments and other lines are removed (Churchman and Schainblatt, 1965, Krauss and Fussell, 1990) and both groups can increase their ability to work towards a common goal. In WS3, $86 \%$ of the participants rated this change as an improvement.

The fifth change was the addition of context to the model, as shown in Figure 14.
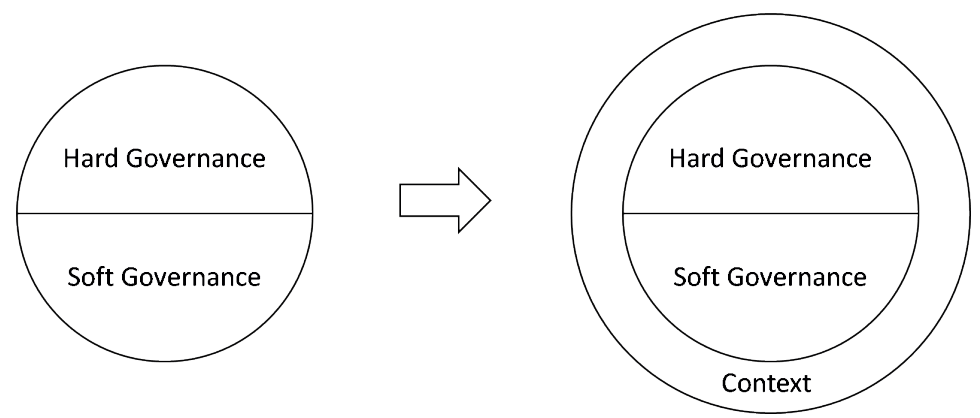

Figure 14 Change of the basic model 
The resulting model adds a third pillar to the initial model: the context. The context of an organisation can be divided into the internal context (within the organisation) and the external context (the environment).

Participants argued that some of the focus areas could be deemed value-free. If a focus area is value-free, it is not possible to improve or grow because the direction of the improvement cannot be determined. These focus areas should be added to the context component as the situational part of the maturity model, as proposed by Mettler and Rohner (2009).

This was discussed with the participants in the third meeting; adding it to the model was proposed in the fourth meeting. In meeting four, the participants were asked to share their opinions on this change. The outcome was that $93 \%$ of the participants preferred the new model to include some context.

The sixth change was to move the focus area "Culture" to the domain of internal "context". In discussions during the third meeting, moving "Culture" to the context component was proposed because culture could be seen as value-free. During meeting four, $86 \%$ indicated their preference for "Culture" being part of the context component. One participant thought that culture has maturity too, as it might be considered desirable. A second participant thought that culture has value and proposed attention to the work of Barrett (1998).

The final change was to move the focus area "Informal organisation" to internal "context". The "informal organisation" could be considered value-free, just like culture. When asked to rate this change in meeting four, $83 \%$ of the participants agreed to move "Informal organisation" to the context component.

As shown in Table 28, the support for the MIG model was $71 \%$. The participants who chose "Different" also agreed that the model was "Ready". However, they wanted to add remarks like "The basic structure is far enough for practice but not complete", or "Every model is a limited reproduction of reality and searching for the right model is an eternal journey. I miss models from social psychology".

Adding these individuals to the total score would yield 85\%. Given that our minimum target percentage of $80 \%$ (as explained before) was thus reached, the series of workshop was stopped after the fourth meeting.

The resulting MIG model is summarised in Table 29. 
The Design of the MIG Model

\begin{tabular}{|c|c|c|}
\hline Governance & Domain & Focus area \\
\hline \multirow[t]{4}{*}{ Soft } & \multirow[t]{2}{*}{ Behaviour } & Continuous improvement \\
\hline & & Leadership \\
\hline & \multirow[t]{2}{*}{ Collaboration } & Participation \\
\hline & & Understanding and trust \\
\hline \multirow[t]{5}{*}{ Hard } & \multirow[t]{2}{*}{ Structure } & Functions and roles \\
\hline & & Formal networks \\
\hline & \multirow[t]{3}{*}{ Process } & IT decision-making \\
\hline & & Planning \\
\hline & & Monitoring \\
\hline \multirow[t]{3}{*}{ Context } & \multirow[t]{2}{*}{ Internal } & Culture \\
\hline & & Informal organisation \\
\hline & External & Sector \\
\hline
\end{tabular}

Table 29 The MIG model version 0.7 (focus areas)

Definitions of the domains are included in Table 30. Definitions of the focus areas are included in Table 31. The definition of the domains and focus areas were adopted from the literature. The source of each definition is included in both tables.

Given that unambiguous and universally accepted definitions for the concepts in the model are unavailable, we shared a list of definitions during the workshops. The definitions were discussed with the participants before they were asked for their opinions regarding any changes or (interim) models.

The initial definitions of the domains and focus areas are defined in Table 30 and Table 31. These definitions were used during the Delphi workshops and in the initial versions of the model. The definitions of the final version are listed in Section 5.6.2.

\begin{tabular}{|l|l|l|}
\hline Domain & Definition & Source \\
\hline Collaboration & Collaboration is defined as making a joint effort towards a goal. & $\begin{array}{l}\text { (de Vreede and } \\
\text { Briggs, 2005) }\end{array}$ \\
\hline Structure & $\begin{array}{l}\text { Structural (formal) devices and mechanisms for connecting and } \\
\text { enabling horizontal contacts, or liaisons, between business and IT } \\
\text { management (decision-making) functions. }\end{array}$ & $\begin{array}{l}\text { (Peterson et al., } \\
\text { 2000, Peterson, } \\
\text { 2004) }\end{array}$ \\
\hline Process & $\begin{array}{l}\text { Formalisation and institutionalisation of strategic IT decision- } \\
\text { making or IT monitoring procedures. }\end{array}$ & $\begin{array}{l}\text { (Peterson et al., } \\
\text { 2000, Peterson, } \\
\text { 2004) }\end{array}$ \\
\hline Behaviour & $\begin{array}{l}\text { Anything that an organism does involving action and response to } \\
\text { stimulation; the response of an individual, group or species to its } \\
\text { environment; the way in which something functions or operates. }\end{array}$ & $\begin{array}{l}\text { (Merriam- } \\
\text { Webster, 2014) }\end{array}$ \\
\hline
\end{tabular}

Table 30 Definitions of the domains (initial) 
Section 5

\begin{tabular}{|c|c|c|}
\hline Focus Area & Definition & Source \\
\hline $\begin{array}{l}\text { Continuous } \\
\text { improvement }\end{array}$ & $\begin{array}{l}\text { A continual stream of innovation to enhance the } \\
\text { value or quality of the products and processes of an } \\
\text { organisation. }\end{array}$ & $\begin{array}{l}\text { Derived from } \\
\text { (Bessant et al., 2001) }\end{array}$ \\
\hline Leadership & $\begin{array}{l}\text { Behaviour that results in supervision, organisation } \\
\text { and change to the life, perceptions, expectations and } \\
\text { values of the members of an organisation. }\end{array}$ & (Burns, 1978) \\
\hline Participation & $\begin{array}{l}\text { Having a part or share in the interaction between the } \\
\text { stakeholders in an organisation. }\end{array}$ & $\begin{array}{l}\text { Derived from } \\
\text { (Van Grembergen et al., 2004) }\end{array}$ \\
\hline $\begin{array}{l}\text { Understanding } \\
\text { and trust }\end{array}$ & $\begin{array}{l}\text { Shared knowledge and confidence between the } \\
\text { stakeholders in an organisation. }\end{array}$ & $\begin{array}{l}\text { Derived from } \\
\text { (Peterson, 2001) }\end{array}$ \\
\hline $\begin{array}{l}\text { Functions and } \\
\text { roles }\end{array}$ & $\begin{array}{l}\text { The organisational hierarchal and non-hierarchal } \\
\text { positions as defined in the organisation. }\end{array}$ & $\begin{array}{l}\text { Derived from } \\
\text { (Van Grembergen et al., 2004) }\end{array}$ \\
\hline $\begin{array}{l}\text { Formal } \\
\text { networks }\end{array}$ & $\begin{array}{l}\text { The formal governing bodies which are part of the } \\
\text { organisation. }\end{array}$ & $\begin{array}{l}\text { Derived from } \\
\text { (Van Grembergen et al., 2004) }\end{array}$ \\
\hline $\begin{array}{l}\text { IT decision- } \\
\text { making }\end{array}$ & $\begin{array}{l}\text { The IT-related decision-making processes, decision } \\
\text { rights and accountability framework. }\end{array}$ & $\begin{array}{l}\text { Derived from } \\
\text { (Weill and Woodham, 2002) }\end{array}$ \\
\hline Planning & $\begin{array}{l}\text { The establishment of goals, policies and procedures } \\
\text { for a social or economic unit. In this model, this is } \\
\text { considered both a top-down and bottom-up process. }\end{array}$ & (Merriam-Webster, 2014) \\
\hline Monitoring & $\begin{array}{l}\text { The monitoring of costs, values and risks of the } \\
\text { continuation and change of the IT services in an } \\
\text { organisation. }\end{array}$ & $\begin{array}{l}\text { Derived from } \\
\text { (Van Grembergen et al., 2004) }\end{array}$ \\
\hline Culture & $\begin{array}{l}\text { The beliefs, values and norms of the members of the } \\
\text { organisation that define how they interact in the } \\
\text { entire organisation, not just IT. }\end{array}$ & (Trenholm and Jensen, 2000) \\
\hline $\begin{array}{l}\text { Informal } \\
\text { organisation }\end{array}$ & $\begin{array}{l}\text { The emergent pattern of social interactions within } \\
\text { the organisation that emerges, rather than being } \\
\text { mandated. This refers to the entire organisation, not } \\
\text { just IT. }\end{array}$ & $\begin{array}{l}\text { (Chan, 2002, Gulati and } \\
\text { Puranam, 2009) }\end{array}$ \\
\hline Sector & $\begin{array}{l}\text { A sociological, economic or political subdivision of } \\
\text { society. }\end{array}$ & (Merriam-Webster, 2014) \\
\hline
\end{tabular}

Table 31 Definitions of the focus areas (initial)

The definitions of the focus areas are based on the literature listed. If a definition was available in these sources, it was adopted. If not, we added a definition similar to the ways in which the focus area was used in the source listed (preceded by "Derived from" in the column "Source"). In a later stage the definitions based on the Marriam-Webster dictionary were replaced by more specific definitions from the literature (see Section 5.6.2). 


\subsection{The design of the maturity levels in the MIG model}

An introduction to maturity models is included in Section 1.3.5 and the research method is described in Section 2.2.4. This section contains a detailed description of the design process.

The basic concept of a maturity model consists of a number of areas - henceforth called focus areas - which mature along a predefined path to achieve higher levels of maturity.

A higher level of maturity is defined as a better means to fulfil its purpose; the predefined path is described by a set of capabilities. Capabilities are the ability to mobilise and deploy resources to achieve a goal (Bharadwaj, 2000).

Most maturity models used for ITG are related to the existing frameworks previously mentioned, which are largely focused on processes and structure (Rogers, 2009). These frameworks make use of different approaches for assessing organisational maturity and performance. Some frameworks, for example COBIT, include a formal maturity model based on the CMM stages (ITGI, 2007). Others, for example ITIL, do not and so require additional frameworks for maturity (de Sousa Pereira and da Silva, 2010). Of these frameworks, only COBIT is truly focused on ITG.

Maturity models in which ITG represents an area can be found more easily. Examples include the IT Capability Maturity Framework from the Innovation Value Institute. This framework is also based on the CMM levels and contains maturity capabilities for IT leadership and governance.

The most dominant foundation of past IS research on maturity modelling is CMM (Pöppelbuß et al., 2011). Perceptions on maturity differ. Some relate maturity to alignment with best practice frameworks. "A maturely governed IT organization is thus defined as an organization that is efficient and aligned with state-of-the-practice frameworks such as COBIT, Val IT or ITIL" (Pöppelbuß et al., 2011).

In a literature review we made an initial selection of maturity models for each focus area. The participants in the Delphi workshops (see Section 5.2) were asked to rate the suitability of the maturity model and received a handout with a summarised description of the capabilities as defined in the selected maturity models.

The maturity models were selected in two rounds (during workshops 3 and 4). After each round the model was improved using the feedback during the meeting. 


\subsubsection{Maturity models}

Maturity can be characterised as "the state of being complete, perfect or ready" (Wendler, 2012, Mettler et al., 2010). Maturity implies evolutionary progress in a specific ability or in the accomplishment of a target from an initial to a desired or normally occurring end stage (Mettler and Rohner, 2009).

Becker et al. (2009) have proposed a generic and well-accepted definition for the maturity model: "A maturity model consists of a sequence of maturity levels for a class of objects. It represents an anticipated, desired, or typical evolution path of these objects shaped as discrete stages". Maturity implies the potential for growth in capability (Paulk et al., 1993).

\section{Type of maturity model}

A systematic mapping study (Wendler, 2012) showed that current maturity model research is applicable to more than 20 domains, and is dominated by software development and software engineering. The study illustrates that issues such as IT alignment, the use of enterprise resource systems, technology and knowledge management, or collaboration processes are all becoming more important. However, the designs of the maturity models "have only been documented very sketchily" (Becker et al., 2009). The aims of maturity models are "raising awareness" of areas of concern and "benchmarking" to compare results across organisations (Maier et al., 2012). Thus, maturity models are helpful in finding better solutions for change, but to become useful they must be applied to a substantial number of companies for valid comparison.

The answer to the question "What makes organisational capabilities mature?" is contingent on the rationale embraced, and tends to be based on the leverage points used in organisational change initiatives (Maier et al., 2012). Maier (Maier et al., 2012) discern four leverage points that have been used in maturity models:

1. Existence and adherence to a structured process;

2. Alteration of organisational structure;

3. Emphasis on people;

4. Emphasis on learning.

The first two points are related to hard governance and the latter two to soft governance. The ways in which processes mature stand in sharp contrast with the ways in which individuals acquire skill (Cooke-Davies and Arzymanow, 2003). The maturity model described in this thesis is a hybrid of all four types. 
The following application-specific purposes of a maturity model are distinguished (De Bruin et al., 2005, Becker et al., 2009, Pöppelbuß and Röglinger, 2011):

- Descriptive, to determine the current state of an organisation with respect to given criteria;

- $\quad$ Prescriptive, if the model indicates how to identify desirable maturity levels and provides guidelines on improvement measures;

- $\quad$ Comparative, if the model can be used for internal or external benchmarking.

The MIG model should preferably be suitable for all three types of purposes.

For IT management and ITG maturity, models represent important instruments because they help find better solutions for change (Becker et al., 2009, Pöppelbuß and Röglinger, 2011). Maturity models are a concept widely used and of considerable relevance to IS practice and IS research (Pöppelbuß and Röglinger, 2011, Becker et al., 2010). To ensure the relevance of maturity models for practice, researchers are further advised to conduct applicability checks with practitioners (Rosemann and Vessey, 2008).

There are critiques on maturity models too: maturity models oversimplify reality and lack empirical foundation; they sometimes define a single path to reach maturity, thereby neglecting potentially advantageous alternative paths; and the procedures and methods that stimulated these models have only been documented very sketchily (Becker et al., 2009, Proença and Borbinha, 2016, Pöppelbuß and Röglinger, 2011). Minimising the limitations of maturity models can be achieved by ensuring continuous and iterative evaluation (Becker et al., 2009, Helgesson et al., 2012).

There is always debate concerning whether higher levels of maturity are better than lower levels (Andersen and Henriksen, 2006). This might not be true for all levels, especially for the highest. This is an issue that will be discussed and eventually solved in a later stage.

Existing maturity models can be divided into three basic types (van Steenbergen et al., 2009):

1. Staged fixed-level models, which distinguish a fixed number of generic levels of maturity, usually around five;

2. Continuous fixed-level models, which differ from the staged fixed-level models in that in the continuous models, focus areas are not attributed to a level, but rather the generic maturity levels are distinguished within each focus area;

3. Focus area models, a type of maturity model based on the incremental improvement of a collection of focus areas to improve a domain. 
Focus area models are much less common than fixed-level models. We share the view that different dimensions have different maturity levels and the assumption of the existence of generic maturity levels is an oversimplification. Thus, we chose to design a focus area maturity model because these provide greater insights on incremental improvement than fixed-level maturity models (van Steenbergen et al., 2010). A distinguishing characteristic of a focus area maturity model is that it also defines the interrelated ways in which focus areas grow in maturity (see Figure 15).

\begin{tabular}{|c|c|c|c|c|c|c|c|c|c|}
\hline Domain & Focus area & 1 & 2 & 3 & 4 & 5 & 6 & 7 & $\ldots$ \\
\hline Domain 1 & Area 1 & & A & & B & & & C & \\
\hline Domain 1 & Area 2 & A & & & & B & & & \\
\hline Domain 2 & Area 3 & & & A & & & B & & C \\
\hline Domain 2 & Area 4 & & & & $A$ & & & B & \\
\hline Domain 2 & Area 5 & & A & & & B & C & & $\mathrm{D}$ \\
\hline & & & & & & & & & \\
\hline
\end{tabular}

Figure 15 Focus area maturity model

Focus area maturity models do not distinguish a fixed number of generic maturity levels, but instead define specific maturity levels for each focus area. The capabilities are numbered A, B, $\mathrm{C}$ and $\mathrm{D}$, as can be seen in the Figure above.

The overall maturity of an organisation is expressed as a combination of the specific maturity levels. The arrows in the right part of the Figure show the interrelated way in which the capabilities can grow between the focus areas. The number of maturity levels is usually somewhere between 10 and 20. The first two columns are the domains and focus areas that are relevant to the topic of the maturity model.

Most maturity models only enumerate maturity levels without considering the situational aspects of the organisational designs (Mettler and Rohner, 2009). Several studies have found that ITG is situational (Rogers, 2009, ITGI, 2011, Sethibe et al., 2007). 
This implies that a one-size-fits-all approach to ITG may not work in all circumstances (Brinkkemper, 1996). Situational maturity models are configured specifically for the (type of) organisation or sector at hand (Mettler and Rohner, 2009).

Thus, we design a situational maturity model. Such a model allows configuration for a specific situation such as the sector or size of an organisation. Engineering a situational method requires standardised building blocks and guidelines, so-called meta-methods, to assemble these building blocks. Situational methods are methods configured specifically for the project at hand (Brinkkemper, 1996).

Situational maturity models have certain benefits, but they have also been criticised. Indeed, critics argue that "the use of method engineering in practice is relatively low [...] due to its inherent complexity" (Fitzgerald et al., 2003). Situational method engineering is also commonly viewed by practitioners as having a costly overhead (Dam and Winikoff, 2013).

Therefore, we opted to change our approach and design a single maturity model and assessment instrument that partly consists of elements suitable for situational versions (e.g. the focus areas in the context).

In a later stage, the resulting artefacts - the MIG model and MIG assessment instrument - can be used to create situational versions.

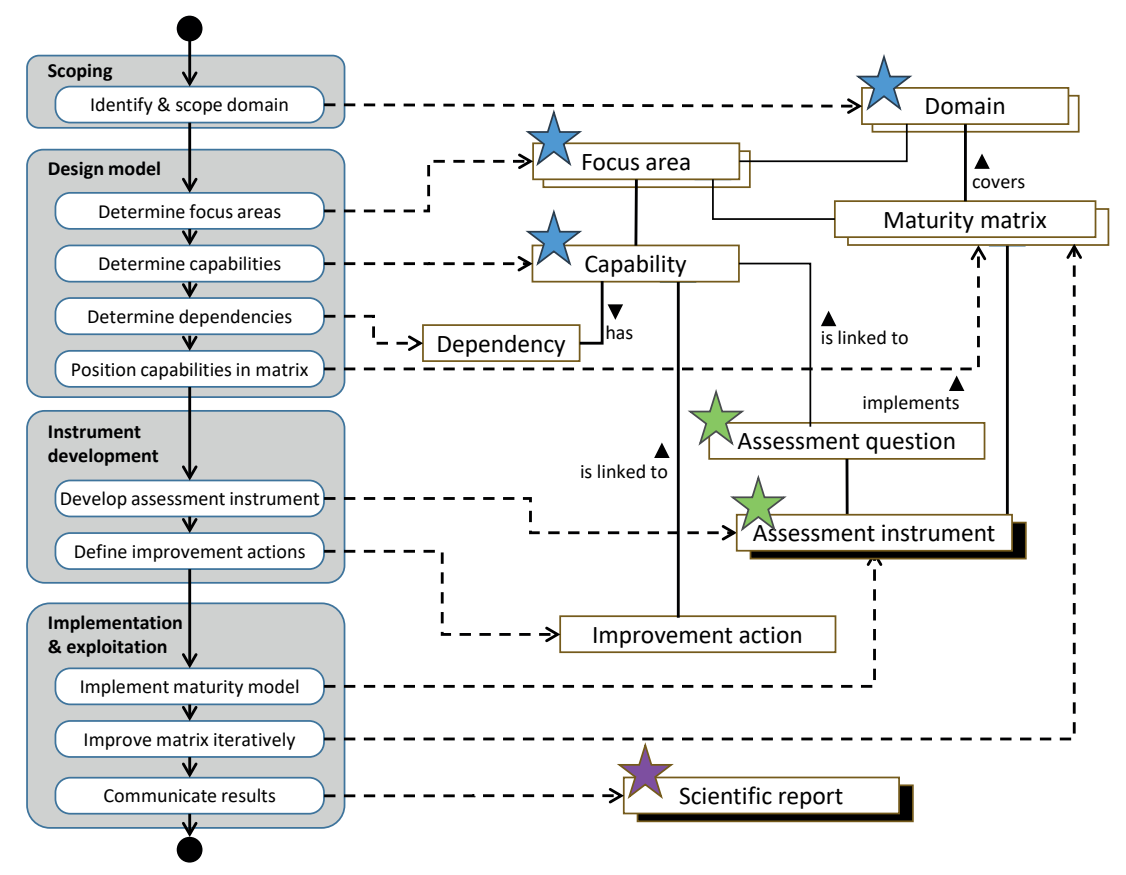

Figure 16 The design of focus area maturity models, adopted from van Steenbergen et al. (2010) 
We initially used the design process described in Figure 16. During the Delphi workshops we were able to define domains, focus areas, capabilities and in a later stage the assessment questions. In Figure 16, the elements discussed in this thesis are marked with three blue stars (MIG model), two green stars (MIG assessment instrument) and one purple star (MIG report).

The following purposes of a maturity model are distinguished (De Bruin et al., 2005, Becker et al., 2009, Pöppelbuß and Röglinger, 2011):

- Descriptive, to determine the current state of an organisation with respect to given criteria;

- $\quad$ Prescriptive, if the model indicates how to identify desirable maturity levels and provides guidelines on improvement measures;

- Comparative, if the model can be used for internal or external benchmarking.

However, it did not prove possible to reach a consensus concerning dependencies between focus areas and maturity levels. In particular, the issue of dependencies between hard and soft governance focus areas stimulated considerable discussion.

Dependencies within the maturity model are a specific characteristic of focus area maturity models. Given that we could not find a solution, we were forced to postpone (see Section 9.5). Another element that was postponed was the definition of improvement actions. Thus, for the time being the MIG instrument can be used to determine current maturity levels, but it does not provide recommended improvement actions.

In this thesis, the building blocks are the focus areas. The situational part of the maturity model is related to focus areas beyond the maturity model. We refer to the state of the environment outside the influence of the maturity model as the "context". Bucher et al. (2007) propose differentiating between "context" and "project type" in situational method engineering. Indeed, "project characteristics must be taken into account as contingencies" to determine which fragments are relevant in a specific situation (Henderson-Sellers and Ralyté, 2010).

Akin to the maturity model, we define the context as a set of focus areas. In our case, the fragments are the focus areas and the "project type" is the ITG maturity of an organisation as defined in the focus area maturity model. The maturity level of the focus areas can be considered stable intrinsic fragments. Intrinsic fragments have a defined value. The stability of a fragment is "particularly important" as it enables the definition of assembly rules and a semantically meaningful comparison of method fragments (Harmsen et al., 1997). 
In particular, context is neglected in existing method engineering approaches (Bucher et al., 2007). In response, in this thesis we include the ITG context. The focus areas of the context can be used to create specific configurations for differences by sector (such as profit and non-profit) or organisational culture (in which clan, adhocracy, market or hierarchy may be dominant, see Section 5.5.4).

\subsubsection{Hard governance}

In the literature, the five-level ranking system introduced for CMM is often used for maturity models for processes. We accordingly selected the maturity levels of CMM for hard governance. The CMM framework was chosen because it is one of the most well-known and widely used maturity models in the IT field.

A CMM-like definition of capabilities in a focus area maturity model means that each focus area will have the following five capabilities (SEI, 2010, Paulk et al., 1991):
A. Initial level;
B. Repeatable level;
C. Defined level;
D. Managed level;
E. Optimising level.

Unlike CMM, the levels will be numbered alphabetically, as shown in Figure 15. The experts were positive about using CMM-like capabilities for hard governance (see Table 32).

\begin{tabular}{|l|c|}
\hline Support for CMM (Paulk et al., 1991) & $\%$ \\
\hline 1. Completely unsuitable & 0 \\
\hline 2. Unsuitable & 0 \\
\hline 3. Indeterminate & 14 \\
\hline 4. Suitable & 57 \\
\hline 5. Completely suitable & 29 \\
\hline Average & 4.2 \\
\hline
\end{tabular}

Table 32 Support for CMM as maturity model for hard governance

With an average score of 4.2 , the participants in the meeting largely agreed on the suitability of CMM-like capabilities for hard governance. 
Not discussed during the meetings was the distinction between continuous and staged representation (Paulk, 1999). In our maturity model, staged representation seemed to fit best. Focus areas include more than just one process and a focus area maturity model does not contain incomplete capabilities. Level 0 in the continuous representation is "Incomplete", while levels 1 to 3 are almost similar to the staged representation.

\subsubsection{Soft governance}

A maturity model for soft governance could not be found. During the first round we tried to select one maturity model for soft governance, just as we did for hard governance. The support for CMM changed dramatically (7\%).

After the second workshop we conducted another literature review. We selected two maturity models as alternatives. The maturity models we selected were Magdaleno et al. 's (2011) Collaboration maturity model and Clark's (2001) Organisational Interoperability maturity model.. The ratings regarding these maturity models were also low: Magdaleno et al. scored a modest 21\%, while support for Clark and CMM was $7 \%$ in both cases. The highest scores were found for "Different" (36\%) and the Likert scale (29\%).

We changed our approach in the following round (workshop 4). The discussion revealed that the participants preferred to select a specific maturity model for each focus area individually. This is in line with the concept of a focus area maturity model (van Steenbergen et al., 2010). It also provided confirmation of the necessity of our choice to design a focus area maturity model as such a type of model support the possibility to define different maturity models for different focus areas.

Where we were unable to find more than one alternative - such as leadership - we selected the model that used a definition of the focus area closest to our definition.

There are large differences between maturity models for leadership, based on the definition adopted. Our definition of leadership concerns behaviour and the characteristics of the leader. Relatively little research has explored the characteristics of leaders whose teams and organisations beat the competition (Kaiser et al., 2008).

An exception is Collins (Collins, 2001), and so we selected this research model. Collins surveyed the Fortune 1000 to identify companies that performed below the average of their business sector for 15 years, and then performed above the average for 15 years. Only 11 companies fit this profile. Their CEOs were found to share two characteristics: a compelling level of modesty and a determination to do whatever must be done to produce the best longterm results, regardless of difficulty. 
It proved difficult to find usable maturity models in the literature for some of the focus areas. For the focus area "Understanding and trust", we could not find a suitable maturity model. We therefore suggested the use of a similar scale, as developed by Reich et al. (1996) in a study regarding understanding between business and IT executives.

The results of the literature review are summarised in Table 33.

\begin{tabular}{|l|l|}
\hline Focus area & Source \\
\hline Continuous improvement & Bessant et al. (2001) \\
\hline Leadership & Collins (2001) \\
\hline Participation & Magdaleno et al. (2011) \\
\hline Understanding and trust & Reich et al. (1996) \\
\hline
\end{tabular}

Table 33 Selected sources (end result)

The sources were intended to be used to deliver descriptions of the capabilities of each focus area.

The participants were asked to rate the perceived suitability $(1=$ completely unsuitable, 5 = completely suitable) of the capabilities defined in the framework for the purpose of the maturity model.

The degree of support for the selected sources is shown in Table 34 until Table 37.

\begin{tabular}{|l|c|}
\hline Support for Bessant et al. (2001) & $\%$ \\
\hline 1. Completely unsuitable & 0 \\
\hline 2. Unsuitable & 0 \\
\hline 3. Indeterminate & 14 \\
\hline 4. Suitable & 43 \\
\hline 5. Completely suitable & 29 \\
\hline Different & 14 \\
\hline Average & 3.6 \\
\hline
\end{tabular}

Table 34 Focus area: Continuous improvement 
Section 5

\begin{tabular}{|l|c|}
\hline Support for Collins (2001) & $\%$ \\
\hline 1. Completely unsuitable & 0 \\
\hline 2. Unsuitable & 7 \\
\hline 3. Indeterminate & 29 \\
\hline 4. Suitable & 43 \\
\hline 5. Completely suitable & 21 \\
\hline Different & 0 \\
\hline Average & 3.8 \\
\hline
\end{tabular}

Table 35 Focus area: Leadership

\begin{tabular}{|l|c|}
\hline Support for Magdaleno et al. (2011) & $\%$ \\
\hline 1. Completely unsuitable & 0 \\
\hline 2. Unsuitable & 14 \\
\hline 3. Indeterminate & 29 \\
\hline 4. Suitable & 57 \\
\hline 5. Completely suitable & 0 \\
\hline Different & 0 \\
\hline Average & 3.4 \\
\hline
\end{tabular}

Table 36 Focus area: Participation

\begin{tabular}{|l|c|}
\hline Support for Reich et al. (1996) & $\%$ \\
\hline 1. Completely unsuitable & 0 \\
\hline 2. Unsuitable & 7 \\
\hline 3. Indeterminate & 14 \\
\hline 4. Suitable & 50 \\
\hline 5. Completely suitable & 29 \\
\hline Different & 0 \\
\hline Average & 4.0 \\
\hline
\end{tabular}

Table 37 Focus area: Understanding and trust

The average suitability for the focus areas as rated by the participants was between 3.4 and 4.0 (on a scale of 5). The focus area "Participation" scored lowest (3.4). One of the participants was of the opinion that Magdaleno et al.'s model could only be used at a micro scale. Two others suggested making a distinction between internal and external participation (the latter is not in scope). 
The focus area "Continuous improvement" scored remarkably highly (3.6), perhaps due to the fact that the name of this focus area was changed several times from "Changeability" to "Adaptability", before settling on "Continuous improvement". It took until the final workshop to reach a consensus. The average suitability of Collins' model was rated 3.8 by the participants.

The maturity levels for "Understanding and trust" are an elaboration of Reich et al.'s (1996) "Scale Used to Measure Understanding of Current Objectives".

A focus area maturity model only contains capabilities. Reich et al. define the first level as "No mission, objectives, or plans have been formulated". Indeed, it describes what is missing and cannot be seen as a capability. Thus, the first level is not included in our maturity model. The average suitability for this focus area as rated by the participants was 4.0. Only the suitability of CMM for hard governance scored more highly.

\begin{tabular}{|c|c|c|}
\hline Area & \# & Capability \\
\hline \multirow{5}{*}{$\begin{array}{l}\text { Hard } \\
\text { governance }\end{array}$} & $\mathrm{A}$ & Processes are usually ad hoc and chaotic. \\
\hline & B & Processes are planned in accordance with policy. \\
\hline & $\mathrm{C}$ & Defined processes are used for managing work. \\
\hline & $\mathrm{D}$ & $\begin{array}{l}\text { Quantitative objectives for quality and process performance, and used } \\
\text { as criteria in managing processes. }\end{array}$ \\
\hline & $\mathrm{E}$ & $\begin{array}{l}\text { An organisation continually improves its processes based on a } \\
\text { quantitative understanding of its business objectives and performance } \\
\text { needs. }\end{array}$ \\
\hline \multirow[t]{4}{*}{$\begin{array}{l}\text { Continuous } \\
\text { improvement }(\mathrm{CI})\end{array}$} & A & $\begin{array}{l}\text { Improvement requires a trigger and implementation is on an ad hoc } \\
\text { basis. }\end{array}$ \\
\hline & B & There is formal commitment to building a system. \\
\hline & $\mathrm{C}$ & $\begin{array}{l}\text { CI behaviour is established at a level local to the wider strategic } \\
\text { concerns of the organisation. }\end{array}$ \\
\hline & $\mathrm{D}$ & Devolve autonomy and empower individuals and groups. \\
\hline \multirow[t]{4}{*}{ Leadership } & A & Contributions through talent, knowledge, skills, and good work habits. \\
\hline & B & Contributes to the achievement of group objectives. \\
\hline & $\mathrm{C}$ & $\begin{array}{l}\text { Organises people and resources towards the effective and efficient } \\
\text { pursuit of predetermined objectives. }\end{array}$ \\
\hline & $\mathrm{D}$ & $\begin{array}{l}\text { Catalyses commitment to and vigorous pursuit of a clear and compelling } \\
\text { vision. Personal humility and professional will. }\end{array}$ \\
\hline \multirow[t]{4}{*}{ Participation } & A & Collaboration is dependent on individual initiative. \\
\hline & B & $\begin{array}{l}\text { The role of the coordinator is needed to centralise and to manage } \\
\text { activities. }\end{array}$ \\
\hline & $\mathrm{C}$ & Group members work in a self-organised and simultaneous manner. \\
\hline & $\mathrm{D}$ & $\begin{array}{l}\text { Group members are aware of the manner in which the group } \\
\text { collaborates during process execution, while process (tacit) knowledge } \\
\text { is shared through ideas, opinions and experiences. }\end{array}$ \\
\hline
\end{tabular}




\begin{tabular}{|l|c|l|}
\hline Area & \# & Capability \\
\hline $\begin{array}{l}\text { Understanding } \\
\text { and trust }\end{array}$ & A & Members cannot identify each other's major current objectives. \\
\cline { 2 - 3 } & B & $\begin{array}{l}\text { Members have a general understanding of each other's current } \\
\text { objectives but cannot identify specific, high-priority objectives. }\end{array}$ \\
\cline { 2 - 3 } & C & $\begin{array}{l}\text { Members understand and are committed to each other's mission, } \\
\text { objectives and plans. }\end{array}$ \\
\hline
\end{tabular}

Table 38 Capabilities of the maturity model (end result)

Based on the stated sources, the capabilities for each focus area can be characterised as described in the table. The first column is the focus area, and the second and third the characterisation of the capability related to different maturity levels (Table 38).

\subsubsection{The context}

For each focus area in the context (except the sector), we selected a framework. The results of the literature review are summarised in Table 39.

\begin{tabular}{|l|l|}
\hline Focus Area & Source \\
\hline Culture & Quinn and Rohrbaugh (1983) \\
\hline $\begin{array}{l}\text { Informal } \\
\text { organisation }\end{array}$ & $\begin{array}{l}\text { Mintzberg (1979); } \\
\text { Cobb (1980); } \\
\text { Galbraith (1974). }\end{array}$ \\
\hline Sector & N/A \\
\hline
\end{tabular}

Table 39 Selected sources (end result)

\section{Culture}

In the literature, frameworks are more common than maturity models. Therefore, many options are available. For organisational culture, the Competing Values Framework (CVF) was selected (Quinn and Rohrbaugh, 1983). The CVF "is probably the most frequently applied framework in the world for assessing culture" (Cameron and Quinn, 2011).

The CVF framework is based on an implicit theoretical framework by which the criteria of organisational effectiveness can be sorted according to three axes or value dimensions:

1. The first value dimension is related to organisational focus, ranging from an emphasis on the internal well-being and development of people to an external emphasis on the well-being and development of the organisation itself;

2. The second value dimension is related to organisational structure, ranging from an emphasis on stability to an emphasis on flexibility;

3. The third value dimension is related to organisational means and ends, ranging from an emphasis on important processes to an emphasis on final outcomes. 
The support for Quinn was measured using the same scale as previously (see Table 40).

\begin{tabular}{|l|l|}
\hline Support for Quinn and Rohrbaugh (1983) & \% \\
\hline 1. Completely unsuitable & 0 \\
\hline 2. Unsuitable & 7 \\
\hline 3. Indeterminate & 21 \\
\hline 4. Suitable & 57 \\
\hline 5. Completely suitable & 14 \\
\hline Different & 0 \\
\hline Average & 3.8 \\
\hline
\end{tabular}

Table 40 Focus area: Culture

This time the scores were 7\% "Unsuitable", 21\% "Indeterminate", 57\% "Suitable" and 14\% "Completely suitable". The remainder were $0 \%$. This resulted in an average suitability of 3.8 as rated by the participants. Thus, in general the response to the CVF framework was positive.

Informal organisation

In terms of the informal organisation, it was less clear which model should be used. We selected three alternatives for discussion during meetings. For "Informal organisation", three alternatives were proposed, along with the option to choose "Something different":

a. The variables outlined by Cobb (1980)

The variables outlined by Cobb were defined as the extent to which the respondent agreed that the target co-worker: was one of the nicest people he/she knew (referent); had good judgement and knew how to get things done in the workplace (expertise); could ensure that others were rewarded for their work (reward); could make things difficult for others (coercive); has legitimate organisational authority over the things done by co-workers (legitimate); had informal lateral influence among work unit peers; and had informal influence on the supervisor (Cobb, 1980).

b. The seven types of lateral relations developed by Galbraith (1974)

The seven lateral relations of Galbraith are: direct contact; liaison roles; taskforces; teams; integrating roles; managerial linking roles; and the matrix organisation (Galbraith, 1974).

c. The Sociogram as described by Mintzberg (1979)

The third alternative was the Sociogram, defined by Mintzberg (1979) as "simply a map of who communicates with whom in an organization, without regard to formal channels". Each alternative was explained in a presentation and discussed during the workshop. 


\section{d. Different}

Participants could choose the option "Different" if none of the proposed alternatives were considered sufficiently appropriate.

For the focus area "Informal organisation" we asked the participants to rate the suitability of the three alternatives (see Table 41).

\begin{tabular}{|l|c|c|c|}
\hline Support for alternative & $\%$ & $\sigma$ & $\#$ \\
\hline a. Five variables, Cobb (1980) & 22.5 & 14.1 & 13 \\
\hline b. Lateral relations, Galbraith (1974) & 26.8 & 22.6 & 14 \\
\hline c. Sociogram, Mintzberg (1979) & 25.7 & 15.6 & 14 \\
\hline d. Different & 25.0 & 36.2 & 5 \\
\hline
\end{tabular}

Table 41 Informal organisation

The second column shows the preference for the sources as a percentage. The third column shows the standard deviation between the answers ( $\sigma$ ). The final column (\#) displays the number of participants who assigned one or more points to a source. On the request of the participants, the option "Different" was added, including the option to explain this choice.

The results indicate no clear preference for one of these alternatives. Nine out of 14 participants assigned zero points to "Different". From this group the average score for Galbraith was 38\%; Mintzberg scored 33\%; and Cobb scored 28\%. The results for the informal organisation did not reveal a clear winner and the subsequent discussion failed to reach a consensus. The practitioners suggested finding out in practice which framework delivers the best results. It is thus clear that this focus area requires additional research.

Sector

During the workshops the focus area "sector" was not mapped onto a model or framework.

\subsubsection{The MIG model v. 0.9}

The end result of the literature review was the intermediate MIG model v. 0.9, summarised in Table 42.

\begin{tabular}{|l|l|l|l|}
\hline \multirow{3}{*}{ Governance } & Domain & Focus area & Maturity model \\
\hline \multirow{3}{*}{ Soft } & \multirow{2}{*}{ Behaviour } & Continuous improvement & Bessant et al. (2001) \\
\cline { 3 - 4 } & \multirow{2}{*}{ Collaboration } & Leadership & Collins (2001) \\
\cline { 3 - 4 } & & Participation & Magdaleno et al. (2011) \\
\cline { 3 - 4 } & & Understanding and trust & Reich and Benbasat (1996) \\
\hline
\end{tabular}


The Design of the MIG Model

\begin{tabular}{|c|c|c|c|}
\hline Governance & Domain & Focus area & Maturity model \\
\hline \multirow{5}{*}{ Hard } & \multirow{2}{*}{ Structure } & Functions and roles & \multirow{5}{*}{$\begin{array}{l}\text { CMM (Paulk et al., 1991) } \\
\text { (used for all five focus areas) }\end{array}$} \\
\hline & & Formal networks & \\
\hline & \multirow{3}{*}{ Process } & IT decision-making & \\
\hline & & Planning & \\
\hline & & Monitoring & \\
\hline \multirow{3}{*}{ Context } & & Culture & Quinn and Rohrbaugh (1983) \\
\hline & Internal & Informal organisation & $\begin{array}{l}\text { There are four alternatives: } \\
\text { a. Five variables: Cobb (1980); } \\
\text { b. Lateral relations, Galbraith (1974); } \\
\text { c. Sociogram, Mintzberg (1979); } \\
\text { d. Something different. }\end{array}$ \\
\hline & External & Sector & Not mapped onto a model or framework. \\
\hline
\end{tabular}

Table 42 The MIG model version 0.9

\subsection{Completing the MIG model}

The design of the MIG model was the result of a Delphi study with four rounds organised between October 2013 and February 2014.

In November 2014 and January 2015 we organised two additional workshops in order to evaluate a solution for the remaining issues with the MIG model, namely:

1. The maturity model for the focus area "Informal organisation";

2. The definition of some focus areas using Webster's dictionary;

3. The design of the first version of the assessment tool (discussed in Section 6).

In this section we summarise some of the results of these workshops. The number of participants at the first meeting was 12 . Only these 12 individuals were thus able to participate in future meetings (see Table 43).

\begin{tabular}{|l|c|c|}
\hline Participation & WS5 & WS6 \\
\hline On location & 10 & 7 \\
\hline Online (afterwards) & 2 & 1 \\
\hline Total & 12 & 8 \\
\hline
\end{tabular}

Table 43 Number of participants for each meeting (WS: workshop number) 
In spite of the request to attend both meetings, some participants were unable to attend the second, similar to the first four workshops.

The average age of the participants was 54 years. The group consisted of one women and 11 men. Eight had a Master's degree, three a Bachelor's degree and one an alternative type of education ('O'). Specific ITG training was attended by eight participants (see Table 44).

By combining the results of the Delphi studies for the domains and focus areas of the ITG maturity model described in Section 5.4 with the maturity models for the focus areas described in Section 5.5, we created version 0.9 of the MIG model (see Table 42).

Two elements that remained unsolved during the first four workshops comprised the model for use as the "Informal organisation" and "Sector". As shown in Table 41, support for each of the initially suggested models for "Informal organisation" was low whereas the model or framework for "Sector" was not discussed.

\begin{tabular}{|c|c|c|c|c|c|c|c|c|}
\hline 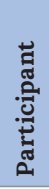 & $\begin{array}{l}\dot{\Xi} \\
\ddot{g} \\
\tilde{J}\end{array}$ & $\underset{<}{0}$ & 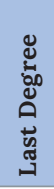 & Current position & $\begin{array}{l}0 \\
\Xi \\
. \Xi \\
0 \\
0 \\
\vdots \\
0 \\
0\end{array}$ & 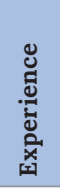 & 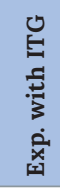 & 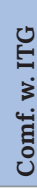 \\
\hline 1 & M & 59 & M & Strategic Adviser & Yes & 36 & 12 & 4 \\
\hline 2 & M & 49 & M & Consultant - Project Manager & No & 25 & 25 & 6 \\
\hline 3 & M & 57 & M & Information Manager CIO Office & Yes & 30 & 30 & 5 \\
\hline 4 & M & 62 & M & Corporate Information Manager & Yes & 10 & 5 & 6 \\
\hline 5 & M & 52 & $\mathrm{O}$ & Information Manager & Yes & 6 & 2 & 3 \\
\hline 6 & M & 36 & M & Information Manager & No & 18 & 2 & 2 \\
\hline 7 & M & 40 & B & Solution Architect & No & 22 & 2 & 1 \\
\hline 8 & M & 66 & B & Management Consultant & Yes & 15 & 15 & 6 \\
\hline 9 & M & 63 & M & Consultant & Yes & 30 & 10 & 6 \\
\hline 10 & M & 60 & B & $<$ No answer $>$ & Yes & 33 & 25 & 6 \\
\hline 11 & $\mathrm{~F}$ & 50 & M & Senior Consultant & Yes & 25 & $10+$ & 7 \\
\hline 12 & $\mathrm{M}$ & 57 & M & Global IT Director & No & 30 & 10 & 7 \\
\hline
\end{tabular}

Table 44 Participant demographics WS5 - WS6 


\subsubsection{In formal organisation}

Version 0.9 of the MIG model does not contain a clear description of the model to be used for the focus area "Informal organisation". Three alternatives have been suggested: variables outlined by Cobb (1980), seven types of lateral relations developed by Galbraith (1974) and the Sociogram described by Mintzberg (1979). All three alternatives are very laborious and not easy to implement. Indeed, we attempted to implement them in some tests, and validated them in workshop 5, but did not succeed. The methods also resulted in considerable discussion during the design of the MIG model. The practitioners failed to reach a consensus and suggested searching for another alternative.

In workshop 6 we proposed an alternative and simple solution that avoided the complexity of adding another framework: to use the nine focus areas of the maturity part of the MIG model. By adding two statements for each of the nine focus areas of hard and soft governance, we expanded the questionnaire to deliver information for the "Informal organisation".

The questionnaire included formal and informal statements for each focus area, such as "Decision-making on IT investments and projects is formally organised". We also added two control statements to check for consistency in the answers of the participants, leading to 20 statements in total.

The support of experts for using statements based on the nine focus areas of the MIG model was high (see Table 45).

\begin{tabular}{|l|c|}
\hline Support for using the nine focus areas & $\%$ \\
\hline 1. Completely unsuitable & 0 \\
\hline 2. Unsuitable & 25 \\
\hline 3. Indeterminate & 13 \\
\hline 4. Suitable & 50 \\
\hline 5. Completely suitable & 13 \\
\hline Different & 0 \\
\hline Average & 3.5 \\
\hline
\end{tabular}

Table 45 Support for using the nine focus areas for informal organisation

Deliberation was noted but on this occasion the practitioners were able to reach a consensus and suggested testing the solution in practice. By using the same focus areas as in the maturity model, we did not add complexity and expected to attain useful information regarding the 
context of each focus area in the FAMM-part of the MIG model. This was appreciated by the practitioners: "It can do no harm to keep it simple", "The simplicity appeals to me".

The support for the proposed statements was somewhat greater (see Table 46):

\begin{tabular}{|l|c|}
\hline Support for proposed statements & $\%$ \\
\hline 1. Completely unsuitable & 0 \\
\hline 2. Unsuitable & 0 \\
\hline 3. Indeterminate & 25 \\
\hline 4. Suitable & 63 \\
\hline 5. Completely suitable & 13 \\
\hline Different & 0 \\
\hline Average & 3.9 \\
\hline
\end{tabular}

Table 46 Support for proposed statements for informal organisation

These statements are used in the first version of the MIG assessment instrument (see the following section).

\subsubsection{Only definitions from literature}

The initial list of definitions of the MIG model included three elements defined using the Merriam-Webster dictionary. Given that these definitions are rather general, we replaced them with definitions from the literature based on a short literature review.

Three definitions are relevant here:

- $\quad$ The domain "behaviour";

- $\quad$ The focus area "planning";

- $\quad$ The focus area "sector".

Behaviour

In this thesis we are interested in the behaviour of the members of an organisation and the behaviour of an organisation as a whole. Historically, organisation theory was focused on the behaviour and attributes of individuals and groups, rather than the organisational aspects of organisational behaviour. In organisation theory exists a discussion concerning the desired focus of organisational behaviour. 
Definitions of organisational behaviour can be divided into three groups: the traditional Big-B and Contextualised-B definitions; and an organisational (Big-O) approach (Heath and Sitkin, 2001). Thus, defining organisational behaviour emphasising interesting individual human behaviour (Big-B). A second definition of organisational behaviour emphasises behaviour in an organisational context (Conceptualised-B). This is the most common way of defining the field of organisational behaviour. A third and more recent view is by emphasising organisational aspects of behaviour, resulting in research that "would devote relatively more attention to topics that help us understand how groups of people organize and carry out their goals (Heath and Sitkin, 2001).

Recent research argues that the organisation itself must also be seen as a social actor (King et al., 2010).

Our definition of "Behaviour" applies to the members of an organisation as well as to the organisation as a social actor. Given that we are interested in behaviour in an organisational context, we use Duncan's (1978) traditional definition of (organisational) behaviour: "a field of study, (that) concerns all aspects of human action in an organizational or group context".

\section{Planning}

Various definitions of planning exist depending on the kind of practice that constitutes the subject of discussion (Alexander, 2016). One popular definition of planning is as an activity "centrally concerned with the linkage between knowledge and organized action" (Friedmann and Hudson, 1974, Hudson et al., 1979).

In our research we use planning in the context of the organisation. For (organisational) planning we adopt Luhmann's (1970) systems theory, which distinguishes between system and environment and the notion that the significance of system building rests not only on the internal ordering of parts into the broader system, but (additionally) in a system's continuous interactions with its environment.

In planning, different systems such as the economy, business administration, IT and law each generate their own version of the organisational environment to be organised or designed (Van Assche and Verschraegen, 2008).

We define "planning as a form of steering aiming to coordinate different systems involved in social organization" (Van Assche and Verschraegen, 2008). These systems can be either inside or part of the environment of an organisation. 
Section 5

\section{Sector}

The researchers know to which organisation and sector the data of each case study belong. In order to analyse data by sector, we require a general classification.

Various statistical classification systems for economic activities, products and goods exist at national, European and international levels. In Europe, the Statistical Classification of Economic Activities in the European Community (Nomenclature statistique des activités économiques dans la Communauté européenne, NACE) has been developed since the 1970s in order to lay the base for the collection and treatment of data with respect to economic activity.

The European Union member states collect their data according to the NACE classification and transfer them to Eurostat, the Statistical Office of the European Union (Schnabl and Zenker, 2013). NACE is based on ISIC, the international classification of economic activities. Both classifications are identical on the highest classification levels, but NACE is more detailed on the lower levels (Eurostat, 2008).

In order to classify the data we use the sections of NACE Rev. 2 (Eurostat, 2008):
A. Agriculture, forestry and fishing
B. Mining and quarrying
C. Manufacturing
D. Electricity, gas, steam and air conditioning supply
E. Water supply; sewerage, waste management and remediation activities
F. Construction
G. Wholesale and retail trade; repair of motor vehicles and motorcycles
H. Transportation and storage
I. Accommodation and food service activities
J. Information and communication
K. Financial and insurance activities
L. Real estate activities
M. Professional, scientific and technical activities
N. Administrative and support service activities
O. Public administration and defence; compulsory social security
P. Education
Q. Human health and social work activities
R. Arts, entertainment and recreation
S. Other service activities 
T. Activities of households as employers; undifferentiated goods- and services-producing activities of households for own use

U. Activities of extraterritorial organisations and bodies

List of definitions (version 1.0)

The definitions of the domains and focus areas of version 1.0 of the MIG model are defined in table 47 and table 48.

\begin{tabular}{|l|l|l|}
\hline Domain & Definition & Source \\
\hline Collaboration & Collaboration is defined as making a joint effort towards a goal. & $\begin{array}{l}\text { (de Vreede and } \\
\text { Briggs, 2005) }\end{array}$ \\
\hline Structure & $\begin{array}{l}\text { Structural (formal) devices and mechanisms for connecting } \\
\text { and enabling horizontal contacts, or liaisons between business } \\
\text { and IT management (decision-making) functions. }\end{array}$ & $\begin{array}{l}\text { (Peterson et al., } \\
\text { 2000, Peterson, } \\
2004)\end{array}$ \\
\hline Process & $\begin{array}{l}\text { Formalisation and institutionalisation of strategic IT decision- } \\
\text { making or IT monitoring procedures. }\end{array}$ & $\begin{array}{l}\text { (Peterson et al., } \\
\text { 2000, Peterson, } \\
2004)\end{array}$ \\
\hline Behaviour & $\begin{array}{l}\text { A field of study (that) concerns all aspects of human action in } \\
\text { an organisational or group context. }\end{array}$ & (Duncan, 1978) \\
\hline
\end{tabular}

Table 47 Definition of the domains (version 1.0) 
Section 5

\begin{tabular}{|c|c|c|}
\hline Focus Area & Definition & Source \\
\hline $\begin{array}{l}\text { Continuous } \\
\text { improvement }\end{array}$ & $\begin{array}{l}\text { A continual stream of innovation to enhance the value or } \\
\text { quality of the products and processes of an organisation. }\end{array}$ & $\begin{array}{l}\text { Derived from } \\
\text { (Bessant et al., 2001) }\end{array}$ \\
\hline Leadership & $\begin{array}{l}\text { Behaviour that results in supervision, organisation and } \\
\text { change to the life, perceptions, expectations and values of the } \\
\text { members of an organisation. }\end{array}$ & (Burns, 1978) \\
\hline Participation & $\begin{array}{l}\text { Having a part or share in the interaction between the } \\
\text { stakeholders in an organisation. }\end{array}$ & $\begin{array}{l}\text { Derived from } \\
\text { (Van Grembergen et } \\
\text { al., 2004) }\end{array}$ \\
\hline $\begin{array}{l}\text { Understanding } \\
\text { and trust }\end{array}$ & $\begin{array}{l}\text { Shared knowledge and confidence between the stakeholders } \\
\text { in an organisation. }\end{array}$ & $\begin{array}{l}\text { Derived from } \\
\text { (Peterson, 2001) }\end{array}$ \\
\hline $\begin{array}{l}\text { Functions and } \\
\text { roles }\end{array}$ & $\begin{array}{l}\text { The organisational hierarchal and non-hierarchal positions as } \\
\text { defined in the organisation. }\end{array}$ & $\begin{array}{l}\text { Derived from } \\
\text { (Van Grembergen et } \\
\text { al., 2004) }\end{array}$ \\
\hline $\begin{array}{l}\text { Formal } \\
\text { networks }\end{array}$ & The formal governing bodies that are part of the organisation. & $\begin{array}{l}\text { Derived from } \\
\text { (Van Grembergen et } \\
\text { al., 2004) }\end{array}$ \\
\hline $\begin{array}{l}\text { IT decision- } \\
\text { making }\end{array}$ & $\begin{array}{l}\text { The IT-related decision-making processes, decision rights } \\
\text { and the accountability framework. }\end{array}$ & $\begin{array}{l}\text { Derived from } \\
\text { (Weill and } \\
\text { Woodham, 2002) }\end{array}$ \\
\hline Planning & $\begin{array}{l}\text { A form of steering aimed at coordinating different systems } \\
\text { involved in an organisation. These systems can be either } \\
\text { inside or part of the environment of an organisation. }\end{array}$ & $\begin{array}{l}\text { (Van Assche and } \\
\text { Verschraegen, 2008) }\end{array}$ \\
\hline Monitoring & $\begin{array}{l}\text { The monitoring of costs, values and risks of the continuation } \\
\text { and change of the IT services in an organisation. }\end{array}$ & $\begin{array}{l}\text { Derived from } \\
\text { (Van Grembergen et } \\
\text { al., 2004) }\end{array}$ \\
\hline Culture & $\begin{array}{l}\text { The beliefs, values and norms of the members of the } \\
\text { organisation that define the way they interact. The entire } \\
\text { organisation, not just IT. }\end{array}$ & $\begin{array}{l}\text { (Trenholm and } \\
\text { Jensen, 2000) }\end{array}$ \\
\hline $\begin{array}{l}\text { Informal } \\
\text { organisation }\end{array}$ & $\begin{array}{l}\text { The emergent pattern of social interactions within the } \\
\text { organisation that emerge rather than are mandated. The } \\
\text { entire organisation, not just IT. }\end{array}$ & $\begin{array}{l}\text { (Chan, 2002, Gulati } \\
\text { and Puranam, 2009) }\end{array}$ \\
\hline Sector & $\begin{array}{l}\text { A grouping of organisations based on the general } \\
\text { characteristics of the goods and services produced as defined } \\
\text { in the sections level of the NACE Rev. } 2 \text { classification of } \\
\text { organisations. }\end{array}$ & (Eurostat, 2008) \\
\hline
\end{tabular}

Table 48 Definition of the focus areas (version 1.0)

The definitions of the focus areas are based on the literature listed. If a definition was available in these sources it was adopted. If not, we added a definition similar to the way in which the focus area was used in the source listed (preceded by "Derived from" in the column "Source"). 


\subsubsection{The MIG model v. 1.0}

The end result of the design process is displayed in Table 49.

\begin{tabular}{|c|c|c|c|}
\hline Governance & Domain & Focus area & Maturity model used \\
\hline \multirow{4}{*}{ Soft } & \multirow{2}{*}{ Behaviour } & Continuous improvement & Bessant et al. (2001) \\
\hline & & Leadership & Collins (2001) \\
\hline & \multirow{2}{*}{ Collaboration } & Participation & Magdaleno et al. (2011) \\
\hline & & Understanding and trust & Reich and Benbasat (1996) \\
\hline \multirow{5}{*}{ Hard } & \multirow{2}{*}{ Structure } & Functions and roles & \multirow{5}{*}{$\begin{array}{l}\text { CMM (Paulk et al., 1991) } \\
\text { (used for all five focus areas) }\end{array}$} \\
\hline & & Formal networks & \\
\hline & \multirow{3}{*}{ Process } & IT decision-making & \\
\hline & & Planning & \\
\hline & & Monitoring & \\
\hline \multirow{3}{*}{ Context } & \multirow[b]{2}{*}{ Internal } & Culture & Quinn and Rohrbaugh (1983) \\
\hline & & Informal organisation & $\begin{array}{l}\text { Based on the nine focus areas of soft and } \\
\text { hard governance. }\end{array}$ \\
\hline & External & Sector & Sections of NACE Rev. 2 (Eurostat, 2008) \\
\hline
\end{tabular}

Table 49 The MIG model version 1.0 (end result)

In order to use the model in practice, we needed to define an assessment instrument. The design of the assessment instrument is described in the following section.

\subsection{Conclusions}

The domains of the resulting MIG model could be seen as an improvement to the contemporary ITG trichotomy. Several studies have demonstrated that soft governance requires greater attention (Rogers, 2009, ITGI, 2011, Davies, 2012, Mettler and Rohner, 2009) and that ITG is situational (Rogers, 2009, ITGI, 2011, Sethibe et al., 2007).

The importance of including both the social aspects of governance and the context as a situational element to a maturity model is thus supported by practice and literature.

This section discusses the answers to our research questions: 
This question is answered by the description of the MIG model. The support following each interim version of the model increased, and after four cycles the consensus of the group was that it was time to test the model in practice. In particular in terms of soft governance, it was challenging to select the right areas and to prevent them from having excessive overlap. During the validation in practice more changes in this part of the model may be required.

The MIG model is the first version of an ITG maturity model for soft and hard governance designed using literature and improved in collaboration with experts from practice.

\section{SQ2.2: What type of maturity model do we need?}

The study highlights the fact that the participants defined different needs for the required maturity model for hard and soft governance. CMM appears adequate for hard governance, but for soft governance it seems less appropriate. This finding is in line with the suggestion by several researchers that CMM does not effectively deal with the social aspects of organisations (Ngwenyama and Nielsen, 2003).

Soft governance requires different maturity models for each focus area. This requirement can be fulfilled by designing a focus area maturity model. The context can be seen as the situational part of the maturity model.

Research by Uehara (2010) has indicated that soft power theory could be applied to COBIT, and more specifically to the processes of COBIT. We intend to apply it to the other domains, too.

\section{SQ2.3: What are the capabilities of each focus area?}

The results of the Delphi study indicate a significant difference between the ways in which hard and soft governance grow in maturity. In the information systems field, MMs are often modelled after CMM. For the hard side of governance this could be achieved in the same manner.

Soft governance revealed a different picture. The literature review highlighted that the use of CMM in this area is less common, and this was confirmed by the practitioners. Furthermore, they agreed that each focus should have its own capabilities based on different maturity models.

The literature review showed that usable maturity models for "Continuous improvement", "Participation" and "Understanding and trust" are scarce. Where required we selected the maturity models closest to the definitions used in the ITG model (Smits and van Hillegersberg, 2014b). For "Leadership", several alternatives are available. When focusing on the behaviour of the leader whose teams and organisations beat the competition, relatively little research is 
available (Kaiser et al., 2008), although Collins (2001) is one of the exceptions. The literature and practice lead to the same conclusion: the ways in which the focus areas of hard and soft governance grow in maturity differ.

For the focus areas in the context we require a framework, rather than a maturity model, in order to access the organisation. For culture and the informal organisation, numerous frameworks are available. Consensus could be reached for the use of the CVF for the focus area "Culture" (Quinn and Rohrbaugh, 1983). For the informal organisation no consensus was reached. The rating by the practitioners for each alternative was approximately equal. Validation in practice is required to ascertain which alternative delivers the best results.

In two additional workshops we validated an alternative solution by using the same focus areas as in the maturity model for the focus area "Informal organisation". This time we reached a consensus. The solution was appreciated by the participants because we did not add complexity and preserved the simplicity of the solution.

\subsection{Summary of this section}

This section contains a detailed description of the design of the MIG model, from the initial version 0.1 until the final version 1.0.

The research method was based on a systematic literature review and six Delphi workshops with practitioners as part of a design science process. The Delphi workshops were conducted using the group decision support system Spilter. The final result, MIG model version 1.0, is summarised in Section 5.6.3.

The following section describes the design of an instrument, based on the MIG model, to measure the current hard and soft governance maturity of an organisation. 


\section{Section 6}

\section{The design and evaluation of the MIG Assessment Instrument}

\subsection{Introduction}

Step III covers the design and evaluation of the MIG model and instrument. An overview of the activities and results discussed in this section is shown in the research outline and marked in light blue in Figure 17.

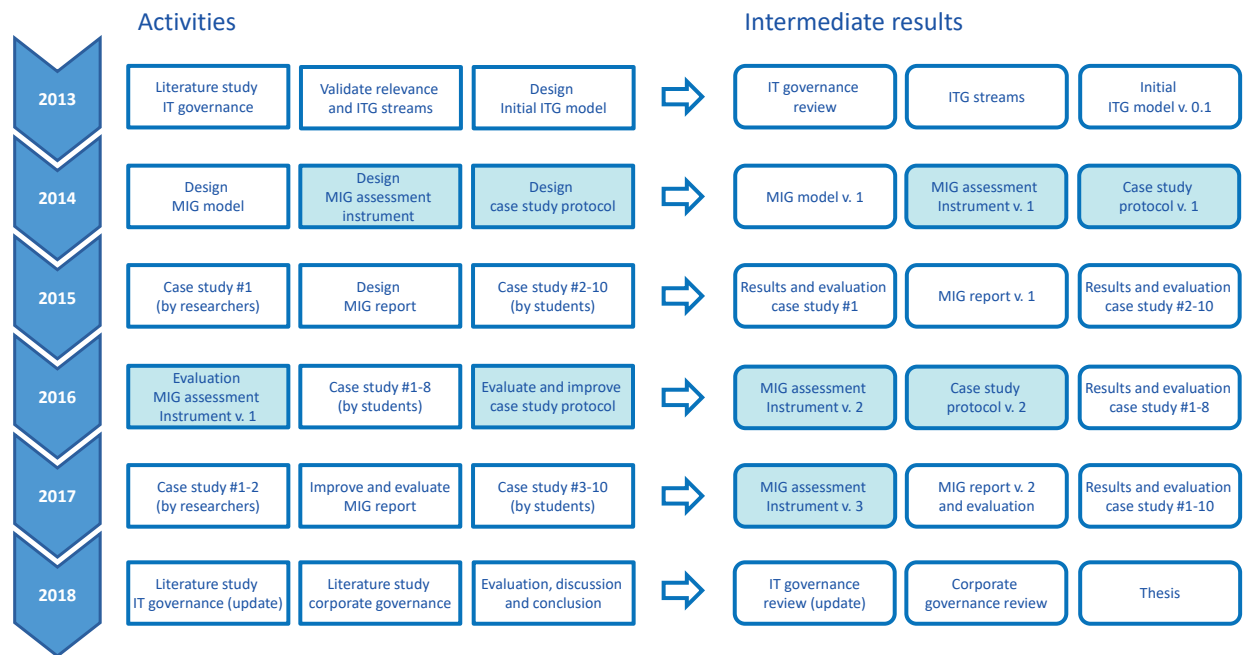

Figure 17 Activities and results of Step III, Part B.

The research method for these activities has been described in Section 2.2. The details of the design process of the MIG assessment instrument and the related results are described in the present section.

The content of this section is based on earlier published work ${ }^{8}$.

8 Smits, D. \& van Hillegersberg, J. 2017. The development of a hard and soft IT governance assessment instrument. International Conference on ENTERprise Information Systems (CENTERIS), Barcelona, Spain. Elsevier, pp 47-54. 
In this section we describe the results of the following two activities:

- $\quad$ The design of the MIG assessment instrument v. 1 until v. 3;

- $\quad$ The design of the case study protocol v. 1 and v. 2.

\subsection{Research method}

The studies described in this section must deliver answers to the following research question:

\section{SQ2.5: How can we develop an assessment instrument based on the MIG model?}

\subsubsection{Designing the MIG instrument}

During the design of the MIG instrument, the guidelines of Hevner were utilised as described in the following enumeration (Hevner et al., 2004):

1. Design as an artefact: The MIG instrument is the artefact.

2. Problem relevance: The relevance of the problem is one of the questions in the case study. The feedback is used to improve the instrument in the next cycle (relevance cycle).

3. Design evaluation: After each use of the instrument, the results are discussed with the interviewee and used as evaluation input in the design cycle. For example, how did the interviewee experience the use of the instrument?

4. Research contributions: Some contributions to the MIG model have been added to make it possible to design the instrument (see Section 5.6).

5. Research rigour: After each use of the instrument, the results are discussed with the interviewee and used as input for the rigour cycle. For example, does the interviewee agree with the results of the instrument?

6. Design as a search process: The first version of the MIG instrument was created and used with the intention of undertaking at least three cycles to improve it.

7. Communication of research: The results of the use of the instrument and the results of the case study are communicated to the participants in the organisation and used as a basis for writing research papers.

We intended to design the MIG assessment instrument based on the MIG model and improve the instrument in (at least) three cycles. More specifically, we will define a new version of the instrument using the data collected in several case studies during an academic year. 


\subsubsection{The case study protocolv. 1 and v. 2}

The purpose of evaluation in design science is to determine if an instantiation of a designed artefact can "establish its utility and efficacy (or lack thereof) for achieving its stated purpose" (Venable et al., 2012).

As long as the instrument was in a development stage we combined the use of the instrument with semi-structured interviews. Interviews are often deemed an essential component of case study research (Burns, 1997). Interviews seek to validate and evaluate (Hevner et al., 2004) whether the results of the instrument correspond with the opinion of the participant and to gather information regarding the reasons why the participant does or does not agree with the resulting maturity level.

For the application of the MIG assessment instrument, we used a case study protocol. The protocol is shown in Figure 18.

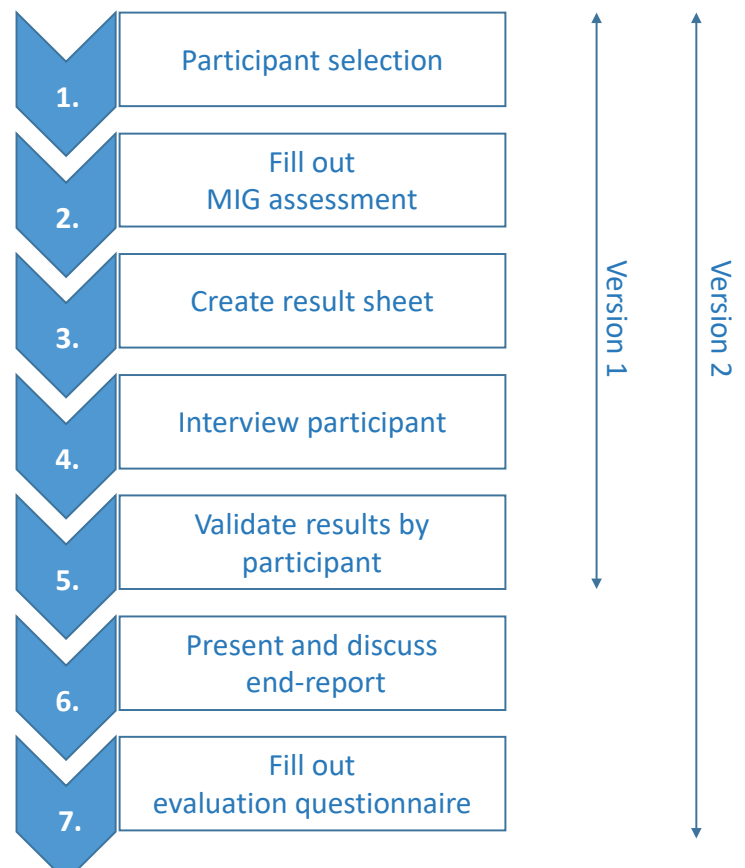

Figure 18 Case study protocol for the MIG assessment 
The protocol used for the application of the instrument was as follows:

1. A group of participants in a strategic role from business and IT were selected and invited to participate in the study.

2. Each participant was asked to fill out the MIG instrument before the interview.

3. The researcher created the results sheet using the instrument and brought it as a handout to the interview.

4. During the semi-structured interview, the results for each focus area were discussed. Where relevant, the results were changed based on the opinion of the interviewee.

Besides the focus areas, three additional questions were asked:

a. Do you miss relevant focus areas?

b. What is your opinion regarding the relevance of our research for hard and soft governance?

c. Do you have anything you would like to add to your feedback?

The interviews lasted an average of one hour and were recorded.

5. Following the interviews, the results were summarised and sent to every participant for validation.

In version 2, the case study protocol was supplemented with steps 6 and 7:

6. A report summarising the results of the study were written, presented and discussed with the client and the participants.

7. The participants (for case studies conducted by the researchers) or students were invited to fill out a short evaluation questionnaire.

Having completed the interviews, the results were combined and analysed. The results of the analysis, conclusions and recommendations were anonymised, summarised in a report and presented to the sponsor of the case study within the organisation. The results of the case studies conducted by the students (cases 3-10) were also presented to the researchers.

The student groups were obligated to present the end results to the researchers and share the completed customer versions of the MIG assessment instrument with the researchers.

The evaluation form used was created based on an evaluation template for expert reviews of maturity models (Salah et al., 2014). The participants were invited to fill out the evaluation questionnaire after the interview, while the students were invited following the presentation of the end results to the researchers. 
The design and evaluation of the MIG Assessment Instrument

\subsubsection{Evaluation strategy}

The first cycle started with the design of the first version of the MIG assessment instrument. As described in Section 2.2.4, each year we introduced minimal changes to the instrument.

The changes applied were the results of obvious inadequacies or improvements suggested by a substantial proportion of the participants.

We intended to use the MIG instrument in case studies. For the design cycle, the MIG model, the MIG instrument and the interviews of the case study are relevant. The intention was that the developed instrument will be used in case studies combined with interviews.

In order to determine the validity of an instrument, "content validity", "construct validity", "reliability" and "internal validity" were to be evaluated (Straub, 1989). Given the qualitative nature of the research, we did not test for the relationship between variables ("statistical conclusion validity").

The purpose of evaluation in design science is to determine if an instantiation of a designed artefact can "establish its utility and efficacy (or lack thereof) for achieving its stated purpose" (Venable et al., 2012). We used the MIG assessment instrument combined with semi-structured interviews to collect data. The interviews were intended to validate and evaluate (Hevner et al., 2004) whether the results of the instrument matched the opinions of the participants and to gather information regarding the reasons why they did or did not agree with the resulting maturity level.

By using diverse data-gathering methods and comparing results, it became possible to determine the extent to which instrumentation affects the findings, as well as their robustness.

The next section (Section 6.3) covers the results of the design of this initial version of the MIG assessment instrument.

\subsection{The first cycle: MIG assessment v. $1(2015)$}

The first version of the MIG assessment instrument was created in the first quarter of 2015 and was based on version 1.0 of the MIG model. The proposed instrument was discussed and evaluated during Delphi workshop 6 (see Section 5.6) with practitioners.

In workshop 6 we shared a handout of the key elements of the maturity levels and the statements for the MIG assessment instrument. Furthermore, we explained the case study protocol and the intention to improve the instrument in several cycles. 
In order to determine whether additional changes in the initial design of the MIG assessment instrument were required, we aimed for $80 \%$ or greater consensus, akin to when designing the MIG model.

Experts' support for the key elements and the statements used in the MIG assessment instrument was high (see Table 50 and Table 51).

\begin{tabular}{|l|c|}
\hline Key elements of MIG assessment instrument & $\%$ \\
\hline 1. Completely unsuitable & 0 \\
\hline 2. Unsuitable & 0 \\
\hline 3. Indeterminate & 0 \\
\hline 4. Suitable & 87 \\
\hline 5. Completely suitable & 13 \\
\hline Different & 0 \\
\hline Average & 4.1 \\
\hline
\end{tabular}

Table 50 Support for key elements MIG assessment instrument

\begin{tabular}{|l|c|}
\hline $\begin{array}{l}\text { Statements regarding MIG assessment } \\
\text { instrument }\end{array}$ & $\%$ \\
\hline 1. Completely unsuitable & 0 \\
\hline 2. Unsuitable & 0 \\
\hline 3. Indeterminate & 13 \\
\hline 4. Suitable & 74 \\
\hline 5. Completely suitable & 13 \\
\hline Different & 0 \\
\hline Average & 4.0 \\
\hline
\end{tabular}

Table $\mathbf{5 1}$ Support for statements MIG assessment instrument

Thus, the workshop resulted in strong support for the initial version of the assessment instrument. We concluded that the proposed instrument could be used as an initial version, and hence there was no need to plan an additional Delphi workshop.

The instrument was used in several case studies conducted by students and the researchers.

We used and improved the instrument in three yearly cycles. In each academic year, we conducted between eight and 10 case studies. The collected data were used to create the improved version for the following year.

Each version of the MIG instrument consists of two parts (Excel sheets): 
A. The first part (A) is intended for the participant and contains the statements and other questions.

B. The second part (B) is for the researcher and is used to create the results sheet.

Both parts are separated to prevent the participant from being influenced by the results when filling out the assessment.

The MIG assessment instrument (Part A) consists of a general form with some demographic information and two questionnaires. The second part for the researcher (Part B) is only used to create the results form.

In order to facilitate the importing of data in Part B of the instrument, a hidden tab was added for the easy transfer of all data from the customer version to the researcher version.

\subsubsection{The MIG assessment instrument (Part A - participant)}

The MIG instrument for the participant consisted of two questionnaires.

Questionnaire 1:

Based on focus areas and maturity models (as described in the MIG model without changes), this questionnaire consisted of:

- $\quad 84$ statements, two for each of the 42 maturity levels in the focus area maturity model part of the MIG model;

- $\quad 20$ statements for the focus area "Informal organisation".

The interviewee was required to decide for each of the 104 statements whether they apply to the current status of the organisation. Participants were able to add a comment or motivation where necessary.

All statements were based on two key elements of the definitions of focus areas and maturity levels in the MIG model. Content analysis was used to determine the key elements in the definitions (Berg et al., 2004). The MIG model cannot meaningfully be researched in reference to only one theory. In such cases, the researcher may have to "synthesise" the existing viewpoints in the literature (Imenda, 2014). The synthesis may be called a model or conceptual framework, which represents the "integrated" view of ITG (Jabareen, 2009).

The conceptual framework was used to select the most relevant elements in the definition of the maturity levels. For each definition, we determined the two (in the opinion of the researchers) most relevant "themes". For example, for the first (or initial) level of CMM we chose 
"Ad hoc" and "Chaotic". The themes were used to create the statements. In order to validate such choices, the instrument was only used in combination with interviews.

In each cycle, the key elements (and statements) were reconsidered to compare the results of the instrument and the interview. The statements were presented in a random order, but grouped by focus areas. Pilots using test versions of the questionnaire with participants revealed that a random order for all statements was considered confusing. Randomising is important to prevent the participant from influencing the results of the assessment before the interview.

For each statement, the participant had to decide whether he or she agreed (yes) or not (no). The basic principle was that if the response is positive for both statements, the level will be reached. Furthermore, it is only possible to reach a level if all preceding levels have been reached.

Thus, to mark a level as reached, two conditions had to be met:

1. Both the statements for a level should be answered positively.

2. All preceding levels must be reached.

Figure 19 presents an example of statements for the focus area "Functions and roles" for MIG assessment instrument version 1. A complete list of statements for MIG assessment instrument version 3 is included in Appendix C (the statements for version 2 and 3 are equal).

\begin{tabular}{|c|c|c|c|}
\hline \multirow{2}{*}{$\begin{array}{l}\text { Focus } \\
\text { Area }\end{array}$} & \multicolumn{3}{|c|}{ Enter ' $y$ ' or ' $Y$ ' in the column 'Fulfillend' if the statement is fulfillend in your organization. Please assess the current situation! } \\
\hline & Statement & Fulfilled & Remarks \\
\hline \multirow{7}{*}{ 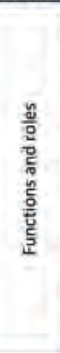 } & Functions and roles exercised by different people are completed in a comparable way. & & \\
\hline & $\begin{array}{l}\text { Functions and roles are described in standards, procedures, tools and methods and communicated in the } \\
\text { organization. }\end{array}$ & & \\
\hline & In the IT organization several functions and roles are defined. & & \\
\hline & $\begin{array}{l}\text { The organization makes use of an incremental and innovative process to continually improve the functions and roles } \\
\text { of the organization. }\end{array}$ & & \\
\hline & The functions and roles are monitored and measured, Action will be taken if functions or roles are not effective. & & \\
\hline & Functions and roles satisfy to a fixed (standardized) pattern. & & \\
\hline & $\begin{array}{l}\text { The organization continually collects information on the quality and performance of the functions and roles of the } \\
\text { organization. }\end{array}$ & & \\
\hline
\end{tabular}

Figure 19 Examples of statements for focus area "Functions and roles" in questionnaire 1 (v. 1)

Questionnaire 2:

For the second questionnaire on culture, we used an existing questionnaire: the Organizational Cultural Assessment Instrument (OCAI). This instrument was developed by Cameron and Quinn (2005) as a means for organisations to quantify organisational culture. 
It fits our purpose as we need a simple instrument to identify the culture and compare organisations. The questionnaire consists of 24 statements, for which six times 100 points have to be divided over six groups of four alternative statements.

\subsubsection{The MIG assessment instrument (Part B - rese a r cher)}

By importing the results of Part A of the instrument into Part B, a results chart can be generated. A simplified example of the results chart is displayed in Figure 20 (fictitious data).

\begin{tabular}{|l|c|c|c|c|c|}
\hline Including check & \multicolumn{5}{|c|}{ Level } \\
\hline Focus area & A & B & C & D & E \\
\hline Continuous & 0 & 0 & 0 & 0 & 0 \\
Leadership & 0 & 0 & 0 & 0 & 0 \\
Participation & 2 & 2 & 2 & 1 & n.a. \\
Understanding and & 0 & 0 & 0 & n.a. & n.a. \\
\hline Functions and roles & 0 & 0 & 0 & 0 & 0 \\
Formal Networks & 1 & 0 & 0 & 0 & 0 \\
IT decision-making & 2 & 0 & 0 & 0 & 0 \\
Planning & 1 & 0 & 0 & 0 & 0 \\
Monitoring & 1 & 0 & 0 & 0 & 0 \\
\hline
\end{tabular}

\begin{tabular}{|l|r|}
\hline Culture & Average \\
\hline Clan & 20,3 \\
Adhocracy & 19,8 \\
Market & 45,3 \\
Hierarchy & 14,5 \\
\hline
\end{tabular}
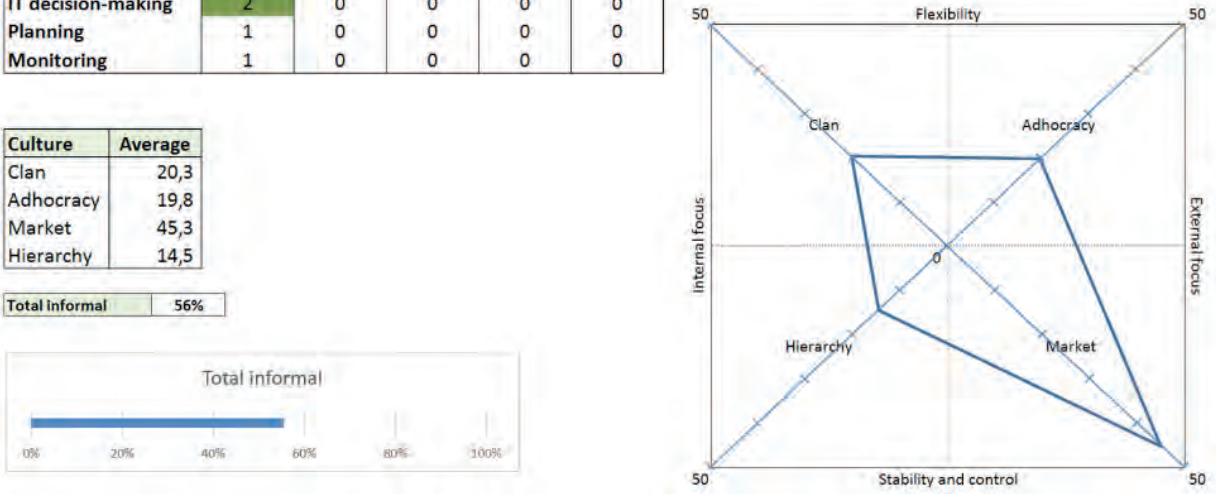

Figure 20 Results of MIG assessment (example; simplified)

The first table, "IT Governance Maturity", displays the results of the maturity levels based on the answers entered by the participants. To mark a level as being reached, two statements (first condition) must be answered positively, including all preceding levels (second condition). If both conditions are met, the field is marked green, indicating that the level is reached. The focus area "Functions and roles" is an example of an unexpected situation: B shows a value of 1, and level $\mathrm{C}$, a value of 2 . This might be an imperfection of the instrument and must be discussed with the participant. Non-existing levels are marked as not available (n.a.). 
The second table and the graph on the right show the organisation's positioning in the Competing Values Framework (Quinn and Rohrbaugh, 1983). The table shows the resulting numerical values.

The final part of the results sheet shows a percentage for "informal organisation". It displays the percentage of positively answered statements out of a total of 18 as a percentage and graph.

\subsection{The second cycle: MIG assessment v. 2 (2016)}

The changes in the second version of the MIG assessment instrument were based on the evaluation during the second cycle. The changes described in this section are summarised in Table 58 and the results of the evaluation are described in the next section (especially 7.3.3).

For the second cycle in 2016, we introduced the following changes to the assessment instrument:

a. Participants complained about the number of questions in the questionnaire. Unfortunately, there was little we could do without choosing a completely different approach. A small improvement, reducing the number of questions, was achieved by removing the questions for the A-level, as this is the minimum or starting level and is always reached, hence no assessment is required.

b. We received numerous comments about "univocal or unclear statements/questions", and were obligated to replace all statements. The initial version included an excessive number of interchangeable terms, potentially instigating different interpretations amongst participants.

The statements were simplified using the same conceptual framework as used for the first version. The main difference between the 2015 and 2016 versions was in the use of the conceptual framework. Indeed, in the 2016 version the language used to complete the statements was kept as simple and consistent as possible.

The conceptual model for the MIG assessment instrument is described in Table 52 . 
The design and evaluation of the MIG Assessment Instrument

\begin{tabular}{|c|c|c|c|}
\hline Focus area & Maturity level $^{9}$ & Key element 1 & Key element 2 \\
\hline \multirow{4}{*}{$\begin{array}{l}\text { Continuous } \\
\text { improvement }\end{array}$} & B & Structured & System \\
\hline & $\mathrm{C}$ & Goal-oriented & Linked to strategy \\
\hline & $\mathrm{D}$ & Proactive & Empowered \\
\hline & $\mathrm{E}$ & Learning mechanism & Unlearning mechanism \\
\hline \multirow[t]{5}{*}{ Leadership } & B & Knowledge & Good work habits \\
\hline & $\mathrm{C}$ & $\begin{array}{l}\text { Contribute to group } \\
\text { objectives }\end{array}$ & Work effectively in groups \\
\hline & $\mathrm{D}$ & Organise & Competent \\
\hline & $\mathrm{E}$ & Catalyse commitment & Stimulate performance \\
\hline & $\mathrm{F}$ & Humble & Willful \\
\hline \multirow[t]{3}{*}{ Participation } & B & Coordinator role & $\begin{array}{l}\text { Basic collaboration } \\
\text { processes }\end{array}$ \\
\hline & $\mathrm{C}$ & Self-organised & $\begin{array}{l}\text { Collaboration including } \\
\text { monitoring and controlling }\end{array}$ \\
\hline & $\mathrm{D}$ & $\begin{array}{l}\text { Share results and } \\
\text { knowledge }\end{array}$ & Formally concluded \\
\hline \multirow[t]{3}{*}{$\begin{array}{l}\text { Understanding } \\
\text { and trust }\end{array}$} & B & Current objectives & $\begin{array}{l}\text { A general understanding of } \\
\text { IT vision }\end{array}$ \\
\hline & $\mathrm{C}$ & $\begin{array}{l}\text { Current high priority } \\
\text { objectives }\end{array}$ & $\begin{array}{l}\text { Understand each other's } \\
\text { IT vision }\end{array}$ \\
\hline & $\mathrm{D}$ & $\begin{array}{l}\text { Committed to each other's } \\
\text { current objectives }\end{array}$ & Shared IT vision \\
\hline \multirow{4}{*}{$\begin{array}{l}\text { Hard } \\
\text { governance }\end{array}$} & B & Repeatable & Based on policy \\
\hline & $\mathrm{C}$ & Defined & Standardised \\
\hline & $\mathrm{D}$ & Managed & $\begin{array}{l}\text { Process performance } \\
\text { measure }\end{array}$ \\
\hline & $\mathrm{E}$ & Continually improve & $\begin{array}{l}\text { Organisational } \\
\text { performance measure }\end{array}$ \\
\hline
\end{tabular}

Table 52 Conceptual framework of the MIG assessment instrument: nine focus areas

An example:

In the definition of level $\mathrm{C}$ for "Goal oriented CI: There is a commitment to linking CI behaviour, established at "local" level to the wider strategic concerns of the organisation", we selected the following two key elements: "linked to strategy" and "goal-oriented".

9 Level A is not included because this is the starting level (see change A). 
We defined two assessment statements for each maturity level. Each statement was based on one of the key elements. For this example, the assessment instrument used the following two statements:

1. The continuous improvement of our organisation is linked to strategy.

2. The continuous improvement of our organisation is goal-oriented.

A complete list of statements for MIG assessment instrument version 3 is included in Appendix C (the statements for version 2 and 3 are equal).

c. The questionnaire of the 2015 version of the instrument consisted of 20 statements for the informal organisation in which the participant had to decide whether he or she agreed (yes or no with an optional comment). The percentage of "informal" was calculated by dividing the number of positively answered questions by 18 ( 20 minus the two control statements to check the consistency in the answers of the participant). The end result was calculated as the number of statements for which the answer was "yes". This result was represented as one percentage for the informal organisation (see the graph at the bottom left in Figure 20).

In the 2016 version we replaced the statements for the "informal organisation" by sets of extreme statements. For each set, 100 points had to be divided. The extra work necessary for the participants was minimal and we were able to collect data regarding differences between hard and soft governance and the focus areas individually.

Given that the structure of the questionnaire for the informal organisation (two numbers between 0 and 100) was different from the first nine focus areas ("yes" or "no"), the number of questionnaires in the assessment instrument was changed from two to three.

With this change, the MIG assessment instrument came to contain three questionnaires:

- A questionnaire for the first nine focus areas;

- A questionnaire for "informal organisation";

- A questionnaire for culture.

The statements were replaced by simpler statements, as for the remainder of the statements. One extreme represented "formal" and the other "informal", using a simple conceptual framework (see Table 53). 
The design and evaluation of the MIG Assessment Instrument

\begin{tabular}{|l|l|l|}
\hline Focus area & Informal & Formal \\
\hline Continuous improvement & Bottom-up initiatives & Management initiatives \\
\hline Leadership & Shown by non-managers & Shown by managers \\
\hline Participation & Informal processes & Formal processes \\
\hline Understanding and trust & Trust informal leaders & Trust their manager \\
\hline Functions and roles & Informal functions and roles & Formal functions and roles \\
\hline Formal networks & Informal networks & Formal networks \\
\hline IT decision-making & Informally organised & Formally organised \\
\hline Planning & Informal planning & Formal planning \\
\hline Monitoring & Informal process & Formal process \\
\hline
\end{tabular}

Table 53 Conceptual framework of the MIG assessment instrument: the informal organisation

By dividing points, participants were able to decide on the weighting factor for the formal and informal organisation. Examples of the resulting assessment statements for the focus areas "IT decision-making", planning and monitoring are displayed in Figure 21.

A complete list of the statements for "informal organisation" is included in Appendix C.5 (the statements for MIG assessment version 2 and 3 are equal). 
Chapter 6

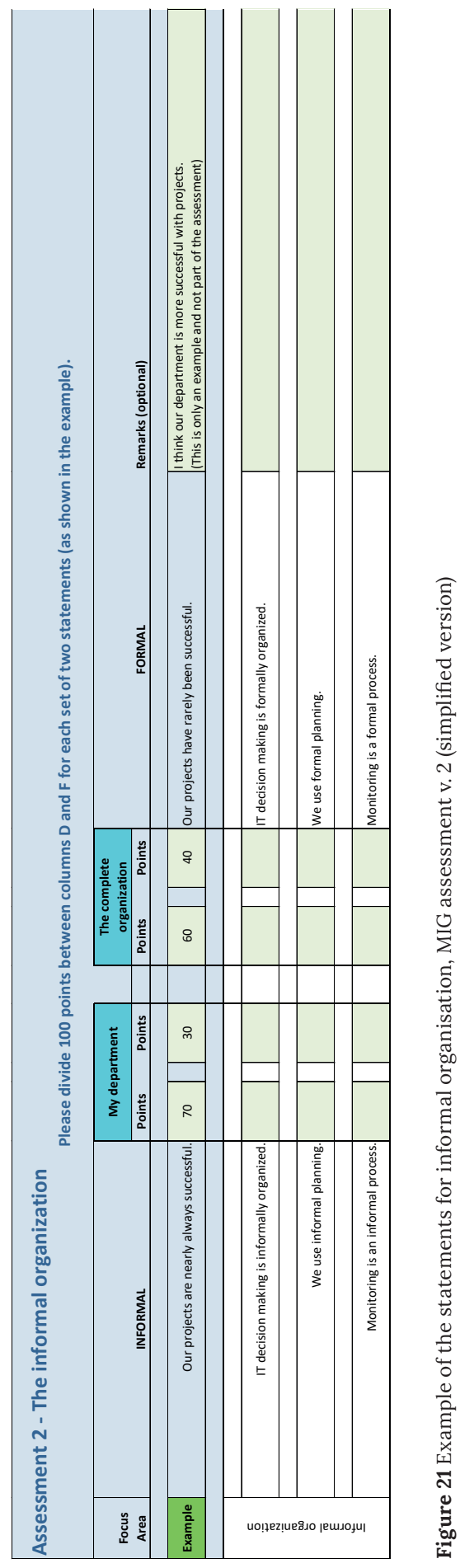




\subsection{The third cycle: MIG assessment v. 3 (2017)}

The changes in the third version of the MIG assessment instrument were based on the evaluation during the third cycle. The changes described in this section are summarised in Table 61 and the results of the evaluation are described in the following section (especially 7.4.3).

For the third cycle in 2017 we introduced the following changes to the assessment instrument: a. In 2015 and 2016 we received numerous comments about there being "too limited choice" where "Yes" and "No" are the only possible responses. The participants sought the ability to add some nuance to their answers.

In 2017 the possible answers to the statements were changed from "Yes" and "No" into a six-point Likert scale using the following percentages: $0,20 \%, 40 \%, 60 \%, 80 \%$ and $100 \%$.

b. A second perspective was added, resulting in a departmental and organisational view (corporate governance view, see Section 8). Thus, in each of the three assessments, the participant was asked to answer the question from a departmental and an organisational perspective. A definition of both perspectives was handed to the participant to be used when filling out the assessment.

As a result of this change, the assessment instrument created two results sheets, one for each view. This change was introduced because participants deemed it easier to answer the questions when they had the ability to compare their own department with other departments of the organisation.

In the case studies, the second view was always used as corporate perspective. An additional benefit of this second view was that it delivered data from a corporate (governance) perspective (see Section 8).

The third version of the MIG assessment instrument is included in Appendix C.

\subsection{Conclusion}

The MIG instrument was based on the MIG model. The MIG assessment instrument was created combining design science (Hevner et al., 2004) and the approach for developing a Focus Area Maturity Model (van Steenbergen et al., 2010).

This section has focused on the development of the first, second and third versions of the MIG assessment instrument. 
The answer to the research question:

\section{SQ2.5: How can we develop an assessment instrument based on the MIG model?}

as described in Section 2.3.1 can be summarised as follows:

In this section, the first, second and third versions of the MIG assessment instrument have been described in detail. The approach and resulting instrument provide an example of an method to design such an assessment instrument and thus attain an answer to research question SQ2.5.

The changes to the instrument constitute the results of the evaluation of the previous version. In the following section, we will discuss the results and evaluation of the use of the first three versions of the MIG assessment instrument through several case studies.

\subsection{Summary of this section}

This section has provided a detailed description of the three cycles of the design of the MIG assessment instrument, including the design of the initial version, the protocol for the use of the instrument and the changes in its second and third versions.

The design of the first version was based on the MIG model and the results of the Delphi workshops with practitioners described in Section 5.

The changes in the second and third versions of the MIG assessment instrument were based on the use, improvement and validation of the instrument in several case studies. This constitutes the focus of Part IV of this thesis. 


\section{Part I V}

\section{Use, improvement and validation of the MIG instrument}

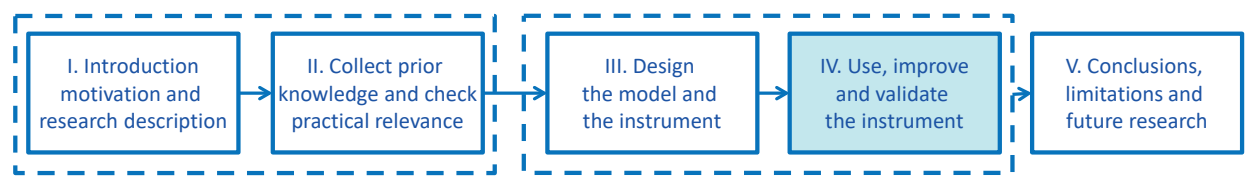

Figure 22 Thesis Part IV. Use, improve and validate the instrument

\section{Section}

7. Results from three cycles of case studies

8. The link between corporate governance and IT governance 


\section{Section 7}

\section{Results from three cycles of case studies}

\subsection{Introduction}

Step IV covers the use, improvement and validation of the MIG assessment instrument. An overview of the activities and results discussed in this section is shown in the research outline and marked in light blue in Figure 23.

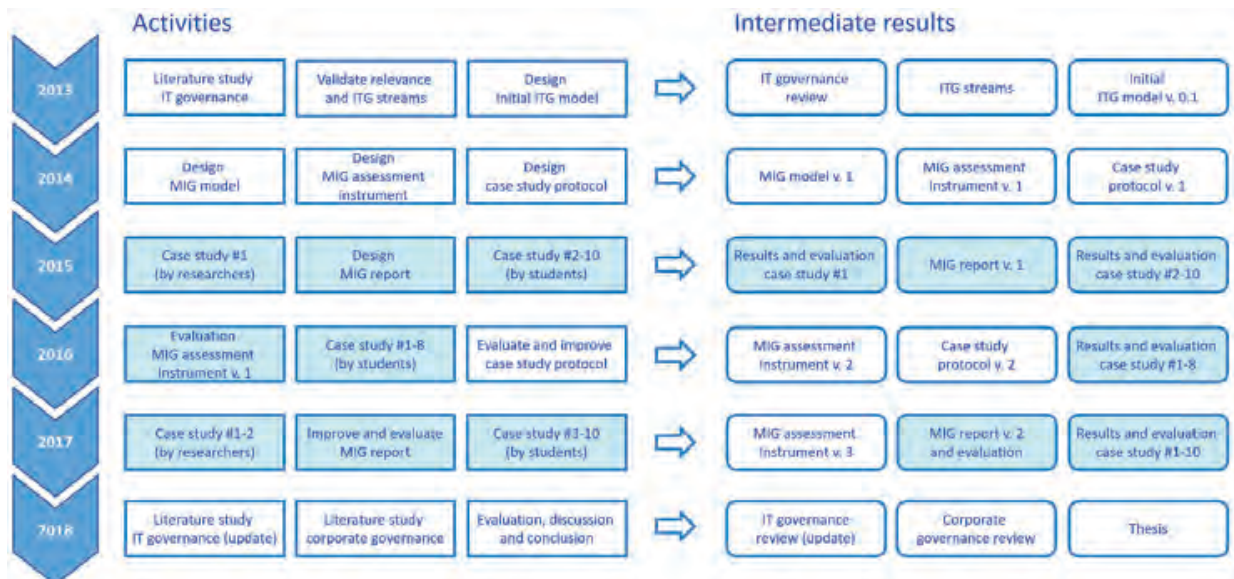

Figure 23 Activities and results of Step IV, Part A.

This section describes the results of the case studies conducted from 2015 until 2017 using the first, second and third versions of the MIG assessment instrument.

The light blue elements are discussed in this section. The research method for these activities was described in Section 2.2. Further details for this specific study and the results are described in the present section. 
The content of this section is partly based on previously published work. ${ }^{10},{ }^{11}$

This section describes the case studies conducted during the first, second and third cycles of the use, improvement and validation of the MIG assessment instrument. In this section we describe the results of the following activities:

- $\quad$ The design of the case study protocols v. 1 and 2;

- $\quad$ The design and evaluation of the MIG report v. 1 and the design of v. 2;

- $\quad$ The results of the case studies in 2015 until 2017;

- $\quad$ The results of the evaluations conducted with participants, students and practitioners.

\subsection{Research method}

The research method is described in Section 2.2.

The studies described in this section seek to deliver answers to the following research questions:

SQ2.4: How can the current hard and soft ITG be measured in an organisation?

SQ3.2: How usable is the instrument for measuring current hard and soft IT governance?

\subsubsection{Evaluation of the MIG model and the MIG assessment instrument}

As described in Section 2.2.2, we used Gregor and Jones' (2007) anatomy of a design theory to evaluate the design of the MIG model and the MIG assessment instrument.

In Table 54 we describe how we aimed to comply with Gregor and Jones' eight components for the design of the MIG model and the MIG assessment instrument.

10 Smits, D. \& van Hillegersberg, J. 2018b. Hard and soft governance: The missing link between corporate and IT governance. Proceedings of the 6th International Conference on Management, Leadership and Governance (ICMLG), Bangkok, Thailand. Academic Conferences Limited, pp 421-430.

11 Smits, D. \& van Hillegersberg, J. 2018a. The continuing mismatch between IT governance maturity theory and practice: a new approach. International Conference on ENTERprise Information Systems (CENTERIS), Lisbon, Portugal. Elsevier, pp 549-560. 


\begin{tabular}{|c|c|}
\hline Component & The way we complied with the component \\
\hline $\begin{array}{l}\text { 1. Purpose and } \\
\text { scope }\end{array}$ & $\begin{array}{l}\text { The MIG model and the MIG assessment instrument intended to increase the } \\
\text { effectiveness of the ITG of an organisation by improving the maturity of the ITG } \\
\text { focus areas. }\end{array}$ \\
\hline 2. Constructs & In the MIG model, ITG is represented by a set of relevant focus areas. \\
\hline $\begin{array}{l}\text { 3. Principles of } \\
\text { form and function }\end{array}$ & $\begin{array}{l}\text { The MIG assessment instrument was created to assess an organisation based on } \\
\text { the MIG model. A case study protocol was described as a guideline for conducting } \\
\text { case studies using the MIG assessment instrument. }\end{array}$ \\
\hline $\begin{array}{l}\text { 4. Artefact } \\
\text { mutability }\end{array}$ & $\begin{array}{l}\text { We conducted our research in three cycles and demonstrated the mutability of } \\
\text { the MIG model and the MIG assessment instrument. }\end{array}$ \\
\hline $\begin{array}{l}\text { 5. Testable } \\
\text { propositions }\end{array}$ & $\begin{array}{l}\text { An element of the case study protocol was that the results of the MIG assessment } \\
\text { instrument were discussed with a participant in an interview. The interviews } \\
\text { intended to test if the results of the instrument corresponded with the opinion of } \\
\text { the participant and to gather information regarding why the participant did or did } \\
\text { not agree with the resulting maturity level. }\end{array}$ \\
\hline $\begin{array}{l}\text { 6. Justificatory } \\
\text { knowledge }\end{array}$ & $\begin{array}{l}\text { The MIG model was designed using existing maturity models from the literature } \\
\text { for each of the focus areas. The MIG assessment instrument was based on the MIG } \\
\text { model and existing definitions of the maturity levels of each focus area. }\end{array}$ \\
\hline \multicolumn{2}{|c|}{ Additional components } \\
\hline $\begin{array}{l}\text { 7. Principles of } \\
\text { implementation }\end{array}$ & $\begin{array}{l}\text { The MIG model is a Focus Area Maturity Model (FAMM). An element of an FAMM is } \\
\text { the definition of improvement actions for each focus area and each maturity level. } \\
\text { These improvement actions are yet to be described but form part of the "Future } \\
\text { research" section of this thesis. }\end{array}$ \\
\hline $\begin{array}{l}\text { 8. Expository } \\
\text { instantiation }\end{array}$ & $\begin{array}{l}\text { We conducted several dozen case studies in three cycles using the MIG model and } \\
\text { the MIG assessment instrument. }\end{array}$ \\
\hline
\end{tabular}

Table 54 Evaluation of the MIG model and the MIG assessment instrument based on Gregor and Jones (2007)

The following sections describe the three design cycles.

\subsection{The first cycle: case studies in 2015}

During the first cycle in 2015 we conducted 10 case studies. Four case studies were conducted by groups of three or four full-time students and five case studies by more experienced part-time students. One case study was conducted by the researchers. In 2015 we used the first version of the MIG assessment instrument.

The case studies were conducted in organisations larger than $1000 \mathrm{FTE}$, or alternatively in the organisation in which the student was employed (in the case of those conducted by the part-time students). At the beginning of the case studies it was agreed that the participating organisations and participants would be anonymous when presenting the results of our research.

Having completed all of the interviews, the results were combined and analysed. The results of the analysis, conclusions and recommendations were all anonymised and presented to the 
sponsor of the case study within the organisation. The results of the case studies conducted by the students (cases 2-9) were additionally presented to the researchers.

\subsubsection{Results}

We only describe the assessment and the interviews in the first phase (as in the other cases).

The case study conducted by the researchers will be described in greater detail.

\begin{tabular}{|c|c|c|c|c|c|c|c|}
\hline \multirow{2}{*}{$\begin{array}{l}\text { \# } \\
1 \\
1\end{array}$} & \multirow{2}{*}{$\begin{array}{l}\text { Sector (NACE v. 2) } \\
\begin{array}{l}\text { O; Public administration and } \\
\text { defence; compulsory social security }\end{array}\end{array}$} & \multirow{2}{*}{$\begin{array}{c}\text { \#P } \\
7 \\
7\end{array}$} & \multicolumn{2}{|c|}{$\begin{array}{l}\text { Informal } \\
\text { (avg.; } \sigma)\end{array}$} & \multirow{2}{*}{$\begin{array}{l}\begin{array}{l}\text { Dominant } \\
\text { culture }\end{array} \\
\text { Clan }\end{array}$} & \multirow{2}{*}{$\begin{array}{l}\text { Size } \\
(\mathbf{x} 1000)\end{array}$} & \multirow{2}{*}{$\begin{array}{l}\text { Improvements } \\
1,2,3,5\end{array}$} \\
\hline & & & $52 \%$ & $10 \%$ & & & \\
\hline 2 & $\begin{array}{l}\mathrm{N} ; \text { Administrative and support } \\
\text { service activities }\end{array}$ & 5 & $39 \%$ & $13 \%$ & Clan & 5.5 & 1,5 \\
\hline 3 & $\begin{array}{l}\text { O; Public administration and } \\
\text { defence; compulsory social security }\end{array}$ & 4 & $57 \%$ & $8 \%$ & Hierarchy & 29.4 & - \\
\hline 4 & $\mathrm{~K}$; Financial and insurance activities & 4 & $33 \%$ & $6 \%$ & Market & 54.0 & 1,2 \\
\hline 5 & $\mathrm{~B}$; Mining and quarrying & 4 & $53 \%$ & $16 \%$ & Hierarchy & 93.0 & 1 \\
\hline 6 & $\mathrm{~K}$; Financial and insurance activities & 3 & $39 \%$ & $10 \%$ & Clan & 0.3 & 1 \\
\hline 7 & $\begin{array}{l}\text { G; Wholesale and retail trade; repair } \\
\text { of motor vehicles and motorcycles }\end{array}$ & 3 & $33 \%$ & $29 \%$ & Market & 3.0 & 1,5 \\
\hline 8 & P; Education & 6 & $59 \%$ & $6 \%$ & Clan & 1.0 & 3,4 \\
\hline 9 & $\begin{array}{l}\text { O; Public administration and } \\
\text { defence; compulsory social security }\end{array}$ & 3 & $61 \%$ & $6 \%$ & Market & 56.5 & 1 \\
\hline \multirow[t]{2}{*}{10} & $\begin{array}{l}\text { I; Accommodation and food service } \\
\text { activities }\end{array}$ & 3 & $35 \%$ & $21 \%$ & Market & 0.8 & 1,4 \\
\hline & Total & 42 & & & & & \\
\hline
\end{tabular}

Table 55 Overview of case studies in 2015

\#

Sector

\#P

Informal

Dominant culture

Size

Improvements is the number of the case study in 2015.

is the sector of the organisation, according to NACE v. 2 (section; description). is the number of participants in the case study. represents the average and standard deviation of the percentage informal governance between the answers of the participants.

is the most dominant culture perspective (highest value). is the number of employees in full-time equivalents (FTE). are the top 5 suggested improvements (see Section 7.3.2).

Appendix F.1 contains a complete overview of the end results of the case studies conducted in 2015. 


\section{Detailed description of case \#1: National government}

This case study was conducted at an independent public body in the Netherlands. The organisation has nationwide coverage and is responsible for one of the key registers in the Netherlands. It employs around 1,500 people at three large locations as well as numerous smaller locations throughout the country.

A selection of eight participants with a strategic business or IT management role were invited to participate in the study. Participants working on all three of the larger locations were involved in this case study.

From this group, seven participated:

- $\quad$ IT: adviser corporate ITG, division manager IT, information manager (2x);

- $\quad$ Business: division manager, chief security officer, head of strategy, and external developments.

One participant was unwilling to complete the MIG assessment questionnaire and was thus excluded from the study.

Table 56 shows the results before and after the interviews.

\begin{tabular}{|c|c|c|c|c|c|c|c|}
\hline \multicolumn{2}{|c|}{ Governance/focus area } & \multirow{2}{*}{$\begin{array}{c}0 \\
6 ; 0\end{array}$} & \multirow{2}{*}{$\begin{array}{c}\mathbf{A} \\
1 ; 4\end{array}$} & \multirow[t]{2}{*}{ B } & \multirow{2}{*}{$\begin{array}{c}\text { C } \\
0 ; 3\end{array}$} & \multirow[t]{2}{*}{ D } & \multirow[t]{2}{*}{$\mathbf{E}$} \\
\hline \multirow{4}{*}{$\begin{array}{ll}\vec{t} & 0 \\
0 & 0 \\
0 & 0\end{array}$} & Continuous improvement & & & & & & \\
\hline & Leadership & $3 ; 1$ & $1 ; 1$ & $1 ; 2$ & $0 ; 1$ & $1 ; 1$ & $1 ; 1$ \\
\hline & Participation & & $5 ; 3$ & $2 ; 3$ & & $0 ; 1$ & \\
\hline & Understanding and trust & $3 ; 2$ & $2 ; 1$ & $2 ; 3$ & $0 ; 1$ & & \\
\hline \multirow{5}{*}{ 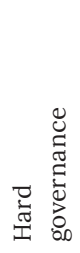 } & Functions and roles & $5 ; 2$ & & $1 ; 2$ & $0 ; 1$ & & $1 ; 2$ \\
\hline & Formal networks & & $3 ; 2$ & $0 ; 2$ & $1 ; 2$ & $2 ; 1$ & $1 ; 1$ \\
\hline & IT decision-making & $2 ; 2$ & $0 ; 1$ & $1 ; 3$ & $1 ; 1$ & $1 ; 0$ & $1 ; 1$ \\
\hline & Planning & $2 ; 1$ & $1 ; 1$ & & $1 ; 3$ & $2 ; 2$ & $1 ; 0$ \\
\hline & Monitoring & & $2 ; 1$ & $2 ; 2$ & $1 ; 3$ & $1 ; 0$ & $1 ; 1$ \\
\hline
\end{tabular}

Table 56 Hard and soft IT governance results before and after the interviews (before; after)

The results summarise the responses from seven participants. The numbers before and after the interviews are separated by a semicolon. The number of available levels is contingent on the focus area (see Table 38).

Two examples:

- $\quad$ For "Continuous improvement", the result of the assessment indicated level 0 on six occasions and level A on one occasion. During the interviews none of the participants 
agreed with level 0. Four participants explained why it should be level A and three why it should be level C. Empty fields reflect levels that were absent from the results sheet of the assessment and the corrected results following the interviews.

- $\quad$ For "Leadership", the result of the assessment showed level 0 on three occasions and levels A, B, D and E on one occasion. During the interviews one participant agreed with level 0. Two participants explained why level B was most appropriate, and four respectively level A, C, D or E. For this focus area, the results of the surveys and opinions differ considerably.

In general, large differences were seen in the results provided by the instrument and the opinions of the participants in terms of soft governance, and fewer distinctions in terms of hard governance.

During the interviews, participants were asked whether they agreed with the results of the assessment, as well as to account for their responses. If the participant was unwilling to agree with the results for one of the focus areas, they were asked to specify the current maturity level using the levels described in a handout. An example of a handout is included in Appendix $\mathrm{E}$, there being minor differences between versions of the MIG assessment instrument.

The motivation for the change provides us with an idea of how participants interpreted the focus areas and the current maturity level. Some participants changed their opinion following an additional explanation of the focus areas. This partly explains the changes.

Table 57 displays the results before and after the interviews for the context.

\begin{tabular}{|c|c|c|c|c|c|c|c|}
\hline \multicolumn{2}{|c|}{ Governance/focus area } & Min. & Max. & Avg. & $\sigma$ & Agree & Not agree \\
\hline \multirow{6}{*}{ 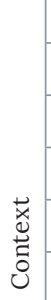 } & \multicolumn{7}{|l|}{ Culture } \\
\hline & - Clan & 24.2 & 38.3 & 33.5 & 4.9 & 7 & 0 \\
\hline & - $\quad$ Adhocracy & 5.8 & 35.0 & 20.4 & 9.2 & 7 & 0 \\
\hline & - $\quad$ Market & 8.3 & 33.3 & 18.6 & 8.6 & 6 & 1 \\
\hline & - $\quad$ Hierarchy & 13.3 & 36.7 & 27.6 & 8.6 & 7 & 0 \\
\hline & Informal organisation & $39 \%$ & $72 \%$ & $52 \%$ & $13 \%$ & 7 & 0 \\
\hline
\end{tabular}

Table 57 Results of IT governance context before and after the interviews

Min, Max, Avg, and $\sigma$

Agree, Not agree are the minimum, maximum, average and standard deviation of the values/percentages between the answers of the participants. show if the participants agree with the result during the interview.

For (organisational) "culture" and "informal organisation", the participants almost unanimously agreed with the results. Regarding "culture", one participant in interview considered the 
value for market too high. This accorded with the opinions of the other participants, whose assessments all showed a lower value. For "Informal organisation", all participants agreed with the results.

Before and after the interviews, we received considerable feedback regarding the usability of the assessment statements, including that the statements "are open to several interpretations". For example, how does one interpret phrases like "programme or hierarchy"? Other remarks included "insufficiently mutually exclusive" and "not easy to fill in without supervision".

The feedback is summarised in the following section, together with the other case studies.

\section{MIG report v. 1}

The results of the assessment were shared with the participating organisation in a so-called MIG report. The MIG report was kept as simple as possible using the data from the assessments and the interviews.

The report structure was as follows:

- $\quad$ A summary of the assessment process and an overview of the participants;

- $\quad$ An anonymous summary of assessment results;

- $\quad$ An estimation of the general maturity level for each of the focus areas;

- A conclusion and recommendation based on the next maturity level for each focus area. The conclusion and recommendations in the MIG report were discussed together with a team of three participants from the organisation. The end result of this discussion was added to the report.

The recommendations were incorporated into the short- and long-term plans of the organisation ("Reisplanner 2016" and "Driejarentraject 2016-2018").

\section{Discussion of the student case studies \#2 - 10 in 2015}

The student case studies were conducted at a heterogeneous set of organisations from government and business. Analysing the student case studies was only feasible for subjects for whom cross-case analysis was meaningful, such as the percentage of "informal organisation", the dominant culture or the usability of the assessment instrument.

Analysis of the remainder of the 35 assessments (42 minus 7 in the first case study resulted in 35 assessments) revealed a pattern comparable to case \#1 (see Appendix F.1). As in the first case study, the results for each participant in the focus area maturity part of the model differed considerably. 
The differences in the context were less pronounced (see Table 57). None of the participants or students challenged the relevance of any of the focus areas in the MIG model. Indeed, most of the reactions to the question "Do you miss relevant focus areas?" were "No", "No, can't think of something" or "It is quite comprehensive". Some suggestions for additional focus areas were made, including "decisiveness", "blocking values and norms", "informal power", "security", "data leakage", "charisma" and "happiness". We evaluated these options but to this point decided to keep the model unchanged, owing to three types of motivations. Below are some examples of each type of motivation:

- $\quad$ The focus area was strongly related with one of the existing focus areas e.g. "charisma" with leadership, "blocking values and norms" with organisational culture or "informal power" with 'informal organisation';

- $\quad$ The focus area was discipline- or content-related e.g. "security" or "data leakage". Based on disciplines constitutes another means of disaggregating ITG, which we initially considered but dropped because it was incongruent with the results of our literature study and Delphi workshops (see Section 3.5);

- $\quad$ The focus area was too general or insufficiently related to ITG e.g. "happiness".

Some participants remarked that the current focus areas covered a very wide area and wondered whether they were instead mere umbrella terms. This might be true; however, it was our aim when designing the MIG model to cover the largest part of the organisational perspective ("hard governance") and social perspective ("soft governance") in a limited number of focus areas.

The results of the assessment for the context ("Informal organisation" and "Organisational culture") were nearly always supported by the participants. Indeed, out of 42 participants, 98\% agreed with the results of "Organisational culture" and all agreed with those of "Informal organisation".

The case studies indicate that the "informal organisation" is relevant in all organisations. The average result for the focus area "Informal organisation" for these ten cases was $47 \%$ (std. $16 \%)$. With one exception, all assessments resulted in a percentage of $11 \%$ or higher.

During all semi-structured interviews, we asked the participants "What is your opinion of the relevance of our research for hard and soft governance?" (see Section 6.2.2). In all interviews conducted by the researchers we received mostly positive (as well as a few neutral) comments regarding the relevance of our research; the students did not ask these three additional questions. For example, "I support it the full 100 per cent" or "It seems to me a good idea. The soft side is at least as important". An example of a neutral remark is "I have yet to see how the results will be used". We consider this neutral because after further explanation it turned out 
that the remark concerned trust in the organisation regarding the actual use of the survey results, and not the survey or research itself.

In contrast, we received numerous comments regarding the assessment instrument. This forms the topic of the following section.

\subsubsection{Evaluation and suggested improvements}

In general, the comments on the MIG instrument were positive regarding its use, but critical in terms of the questioning: "I give it my wholehearted support", "I think it is a good thing. With only the hard side you might get only half [part of ITG]." The case studies resulted in a long list of proposed improvements to the instrument. Some were important and others minor. These suggested improvements were analysed and categorised.

The top 5 suggested improvements for the MIG assessment instrument were based on the responses in Spilter and during the interviews:

1. Univocal or unclear statements/questions in the MIG assessment instrument v. 1.

2. The number of assessments per organisation is too low: there are between three and seven participants. There are too few participants to draw conclusions regarding an entire organisation.

3. The result of the MIG assessment instrument is not in line with the opinion of the participant. During the interviews, participants suggested multiple corrections to the results.

4. With only one percentage, the results sheet presents insufficient data regarding the focus area "Informal organisation". Participants would be interested in more data e.g. the difference between hard and soft governance.

5. Completing the questionnaire is very time-consuming. A shorter questionnaire would be appreciated.

Table 55 presents an overview of the case studies in which these improvements were mentioned.

\subsubsection{Preparing for the next cycle}

As explained in Section 6.2.3, we altered the instrument only minimally for obvious inadequacies or improvements suggested by a substantial proportion of the participants. 
An overview of the changes for the next cycle:

\begin{tabular}{|c|c|c|}
\hline Top 5 suggested improvements & Change? & Motivation \\
\hline $\begin{array}{l}\text { 1. Univocal or unclear statements/ } \\
\text { questions. }\end{array}$ & Yes & Improve the statements/questions. \\
\hline $\begin{array}{l}\text { 2. The number of assessments per } \\
\text { organisation is too low. }\end{array}$ & No & $\begin{array}{l}\text { In the development stage the number of } \\
\text { assessments is sufficient. }\end{array}$ \\
\hline $\begin{array}{l}\text { 3. Focus areas maturity model: result } \\
\text { is not in line with the opinion of the } \\
\text { participant. }\end{array}$ & No & $\begin{array}{l}\text { This can be true, hence we combined the } \\
\text { assessments with interviews. }\end{array}$ \\
\hline $\begin{array}{l}\text { 4. Informal organisation: too little data, } \\
\text { only one percentage. }\end{array}$ & Yes & $\begin{array}{l}\text { Change this part of the assessment to } \\
\text { deliver more data. }\end{array}$ \\
\hline $\begin{array}{l}\text { 5. Completing the questionnaire is very } \\
\text { time-consuming. }\end{array}$ & Yes & $\begin{array}{l}\text { This cannot be solved completely, but we } \\
\text { plan to remove unnecessary statements }\end{array}$ \\
\hline
\end{tabular}

Table 58 Selected changes for the MIG assessment instrument after the first cycle

The selected improvements were discussed in Section 6.4.

\subsection{The second cycle: case studies in 2016}

This section describes the results of eight case studies conducted during the second cycle of the use, and the improvement and validation of MIG assessment instrument version 2.

All studies were conducted in organisations larger than 1000 FTE.

Six were conducted by groups of three or four full-time students, and two (\#7 and \#8) by individual students. 


\subsubsection{Results}

\begin{tabular}{|c|l|c|c|c|l|l|l|}
\hline \# & Sector & \#P & \multicolumn{2}{|c|}{$\begin{array}{l}\text { Informal } \\
(\mathbf{a v g} ; \boldsymbol{)}\end{array}$} & Culture & Size (x1000) & Improvements \\
\hline 1 & $\begin{array}{l}\text { N; Administrative and support } \\
\text { service activities }\end{array}$ & 3 & $49 \%$ & $7 \%$ & Clan & 3.9 & $1,4,5$ \\
\hline 2 & $\begin{array}{l}\text { N; Administrative and support } \\
\text { service activities }\end{array}$ & 4 & $41 \%$ & $6 \%$ & Clan & 5.5 & - \\
\hline 3 & $\begin{array}{l}\text { K; Financial and insurance } \\
\text { activities }\end{array}$ & 4 & $31 \%$ & $6 \%$ & Hierarchy & 1.6 & - \\
\hline 4 & $\begin{array}{l}\text { Q; Human health and social } \\
\text { work activities }\end{array}$ & 7 & $37 \%$ & $5 \%$ & Clan & 3.1 & - \\
\hline 5 & $\begin{array}{l}\text { K; Financial and insurance } \\
\text { activities }\end{array}$ & 3 & $25 \%$ & $8 \%$ & Hierarchy & 1.3 & $1,2,4$ \\
\hline 6 & $\begin{array}{l}\text { D; Electricity, gas, steam and } \\
\text { air conditioning supply }\end{array}$ & 4 & $44 \%$ & $9 \%$ & Clan & 64 & 4,5 \\
\hline 7 & P; Education & 5 & $37 \%$ & $6 \%$ & Clan & 2.7 & $1,2,3,4$ \\
\hline 8 & P; Education & 33 & $50 \%$ & $7 \%$ & Hierarchy & 4.4 & 1,2 \\
\hline & Total & & & \\
\hline
\end{tabular}

Table 59 Overview of case studies in 2016

$\begin{array}{ll}\# & \text { is the number of the case study in } 2016 . \\ \text { Sector } & \text { is the sector of the organisation, according to NACE v. } 2 \text { (section; description). } \\ \text { \#P } & \text { is the number of participants in the case study. } \\ \text { Informal } & \begin{array}{l}\text { represents the average and standard deviation of the percentage informal } \\ \text { governance between the answers of the participants. }\end{array} \\ \text { Dominant culture } & \begin{array}{l}\text { is the most dominant culture perspective (highest value). } \\ \text { is the number of employees in full-time equivalents (FTE). } \\ \text { Improvements }\end{array}\end{array}$

Appendix F.2 contains a complete overview of the end results of the case studies conducted in 2016.

The top 5 suggested improvements for the MIG assessment instrument were based on the responses in Spilter and during the interviews:

1. Most of the questions are hard to answer using just a "Yes"/"No" answer. Some sort of scale, e.g. party or completely (dis)agree, would be helpful.

2. It is unclear to which organisational entity the question refers. An option would be to define two views, e.g. a single department and an entire organisation.

3. The questions are too general. There are differences between departments within an organisation. 
4. Some questions are too complex or unclear. Several terms used in the questions are unclear.

5. The results of the assessment and the interview are different. The interview should be leading.

\section{Discussion of the results of the case studies in 2016}

Just as in the case of the first version of the assessment instrument, the results of the assessment and the interviews differed considerably for the maturity part of the MIG model (see Appendix F.2).

With the exception of some focus areas, the resulting maturity levels varied substantially. The focus area for which this applied differed among the case studies. This may have been due to the specific experiences of a participant regarding a focus area. For example, when answering questions about planning, participants might have some personal positive of negative experience of a project, potentially influencing their opinions regarding the performance of the department in general.

This might have been exacerbated by the number of participants in most case studies. Indeed, we conducted case studies with relatively few participants (between three and seven). Larger numbers of participants might have resulted in less variation in the results.

Nevertheless, the purpose of the research was to design, validate and evaluate the usability of the instrument. In time it would be interesting to conduct case studies with a larger number of participants. Given the interviews and the early design of the instrument, we conducted case studies with small numbers of participants. At this stage, limited numbers of participants were sufficient. In the future, conducting case studies with a larger number of participants would be interesting.

The results of the context part, the focus areas "Informal organisation" and (organisational) "culture" only revealed (very) small differences in relation to the results of the assessment instrument and the opinions of the participants. This finding corresponds with the results of 2015.

\subsubsection{Evaluation of the changes in 2016}

The case studies of 2016 resulted in a list of suggested improvements (see following subsection). However, first we discuss the results of the changes applied in 2016. 


\begin{tabular}{|c|c|c|c|c|}
\hline Changes in 2016 & Discussion of the change & Improved? & Critique & Keep \\
\hline $\begin{array}{l}\text { 1. Univocal } \\
\text { or unclear } \\
\text { statements/ } \\
\text { questions. }\end{array}$ & $\begin{array}{l}\text { Fewer comments on the statements/ } \\
\text { questions were made and were also different } \\
\text { from the first cycle. } \\
\text { We did not receive any remarks about } \\
\text { univocal statements, but some questions } \\
\text { were considered difficult or excessively } \\
\text { general. This might be improved by adding } \\
\text { additional or better documentation. } \\
\text { We conclude that the change can be seen as } \\
\text { an improvement. }\end{array}$ & $\begin{array}{l}\text { Yes, partly } \\
\text { resolved. }\end{array}$ & $\begin{array}{l}\text { We received } \\
\text { no critique } \\
\text { regarding the } \\
\text { change. }\end{array}$ & Yes \\
\hline $\begin{array}{l}\text { 4. Informal } \\
\text { organisation: } \\
\text { too little } \\
\text { data; only one } \\
\text { percentage. }\end{array}$ & $\begin{array}{l}\text { We changed this part of the assessment to } \\
\text { deliver more data. In all cases, the collected } \\
\text { data were accepted by the participants } \\
\text { during interview. The change can be } \\
\text { considered an improvement. }\end{array}$ & $\begin{array}{l}\text { Yes, fully } \\
\text { resolved. }\end{array}$ & $\begin{array}{l}\text { We received } \\
\text { no critique } \\
\text { regarding the } \\
\text { change. }\end{array}$ & Yes \\
\hline $\begin{array}{l}\text { 5. Completing } \\
\text { the } \\
\text { questionnaire } \\
\text { is very time- } \\
\text { consuming. }\end{array}$ & $\begin{array}{l}\text { We removed unnecessary statements but } \\
\text { could not solve the issue completely. } \\
\text { As the instrument delivers more data with } \\
\text { less effort, the change can be deemed an } \\
\text { improvement. }\end{array}$ & $\begin{array}{l}\text { Yes, small } \\
\text { decrease } \\
\text { in number } \\
\text { of } \\
\text { questions. }\end{array}$ & $\begin{array}{l}\text { We received } \\
\text { no critique } \\
\text { regarding the } \\
\text { change. }\end{array}$ & Yes \\
\hline
\end{tabular}

Table 60 Evaluation of the changes in 2016

$\begin{array}{ll}\text { Improved } & \text { Related comments decrease or stop completely } \\ \text { Critique } & \text { Critique regarding the change? } \\ \text { Keep } & \text { Keep the change? }\end{array}$

During the evaluation, we used two questions to determine whether a change should be retained:

1. Does the (number of) comments that resulted in the change decrease or stop completely?

2. Do we receive repeated comments that can be interpreted as critique regarding the change?

If both statements were evaluated positively we decided to keep the change in the subsequent version. the evaluation for each change was positive, the column Keep in Table 60, there was no reason to reverse one of the changes.

Based on the feedback of the participants and the users of the instrument (the students) we conclude that each change can be seen as an improvement.

\subsubsection{Preparing for the next cycle}

As explained in section 6.2 .3 we change the instrument only minimally for obvious inadequacies or improvements suggested by a substantial part of the participants. 
An overview of the changes for the next cycle:

\begin{tabular}{|c|c|c|}
\hline Top 5 suggested improvements & Change? & Motivation \\
\hline $\begin{array}{l}\text { 1. Most of the questions are difficult to } \\
\text { answer using just a "Yes"/"No" answer. }\end{array}$ & Yes & $\begin{array}{l}\text { We introduced a six-point Likert scale } \\
\text { representing 0,20, 40,60, } 80 \text { or } 100 \% \\
\text { agree. }\end{array}$ \\
\hline $\begin{array}{l}\text { 2. It is not clear to which organisational } \\
\text { entity the question refers. An option } \\
\text { would be to define two views, e.g. a single } \\
\text { department and an entire organisation. }\end{array}$ & Yes & $\begin{array}{l}\text { We introduced two views as suggested: a } \\
\text { view for a single department and a view } \\
\text { for the entire organisation. }\end{array}$ \\
\hline 3. The questions are too general. & No & $\begin{array}{l}\text { We will not change the questions but } \\
\text { improve the documentation of the } \\
\text { instrument. }\end{array}$ \\
\hline $\begin{array}{l}\text { 4. Some questions are too complex or } \\
\text { unclear. }\end{array}$ & Yes & $\begin{array}{l}\text { Improve the documentation of the } \\
\text { instrument. }\end{array}$ \\
\hline $\begin{array}{l}\text { 5. The results of the assessment and the } \\
\text { interview are different. The interview } \\
\text { should be leading. }\end{array}$ & No & $\begin{array}{l}\text { We know this, hence we combined the } \\
\text { assessments with the interviews. }\end{array}$ \\
\hline
\end{tabular}

Table 61 Selected changes for the MIG assessment instrument after the second cycle

An explanation of the selected improvements can be found in Section 6.4.

\subsection{The third cycle: case studies in 2017}

This section discusses the use and evaluation of the third version of the MIG assessment instrument. This third cycle of case studies is based on data collected in 10 case studies. Two case studies were conducted by the researchers and eight case studies by the students.

During the preparation of the third cycle we additionally created an evaluation form based on an evaluation template for expert reviews of maturity models (Salah et al., 2014).

The participants were invited to fill out the evaluation questionnaire following interview, while the students were invited to complete it after presenting the end results to the researchers.

\subsubsection{The MIG assessment instrument, version 3}

In order to complement the instrument with a corporate perspective, we ensured not to make significant alterations to the validated instrument (Straub, 1989): the questions were not changed, and most focus areas were not specifically IT-related. Where IT was mentioned (such as in "IT decision-making"), it was replaced by a corporate perspective, defined as "the entire organisation". 
Corporate governance is IT- and business-related. In practice there are almost no IT-specific projects: with the exception of some very particular technical projects, all projects are businessrelated. In the assessment, the participants were asked to fill out the questionnaire from both a departmental and corporate perspective. We explained that for "the entire organisation", the focus area "IT decision-making" may be seen as "Decision-making".

During the interviews, we evaluated the results sheet for both perspectives. The only change to the instrument was to double the questionnaires by adding a second view to the instrument for the corporate governance perspective.

The adjusted instrument consisted of three questionnaires:

- Questionnaire 1: containing 70 statements using a six-point Likert scale for the department and for the corporate perspective (the entire organisation).

- Questionnaire 2: containing nine groups of two statements. Respondents had to divide 100 points between each pair. Twice, again for the department and for the entire organisation.

- Questionnaire 3: the third questionnaire on culture was based on an existing questionnaire, the Organizational Cultural Assessment Instrument (OCAI). The respondents filled out the questionnaire twice, once for each perspective.

When processing the results, we created two results sheets rather than one. Each sheet displayed the maturity level reached for each of the nine focus areas, a percentage for "informal organisation", and the positioning within the Competing Values Framework for one of the perspectives (see Figure 24). 
Section 7
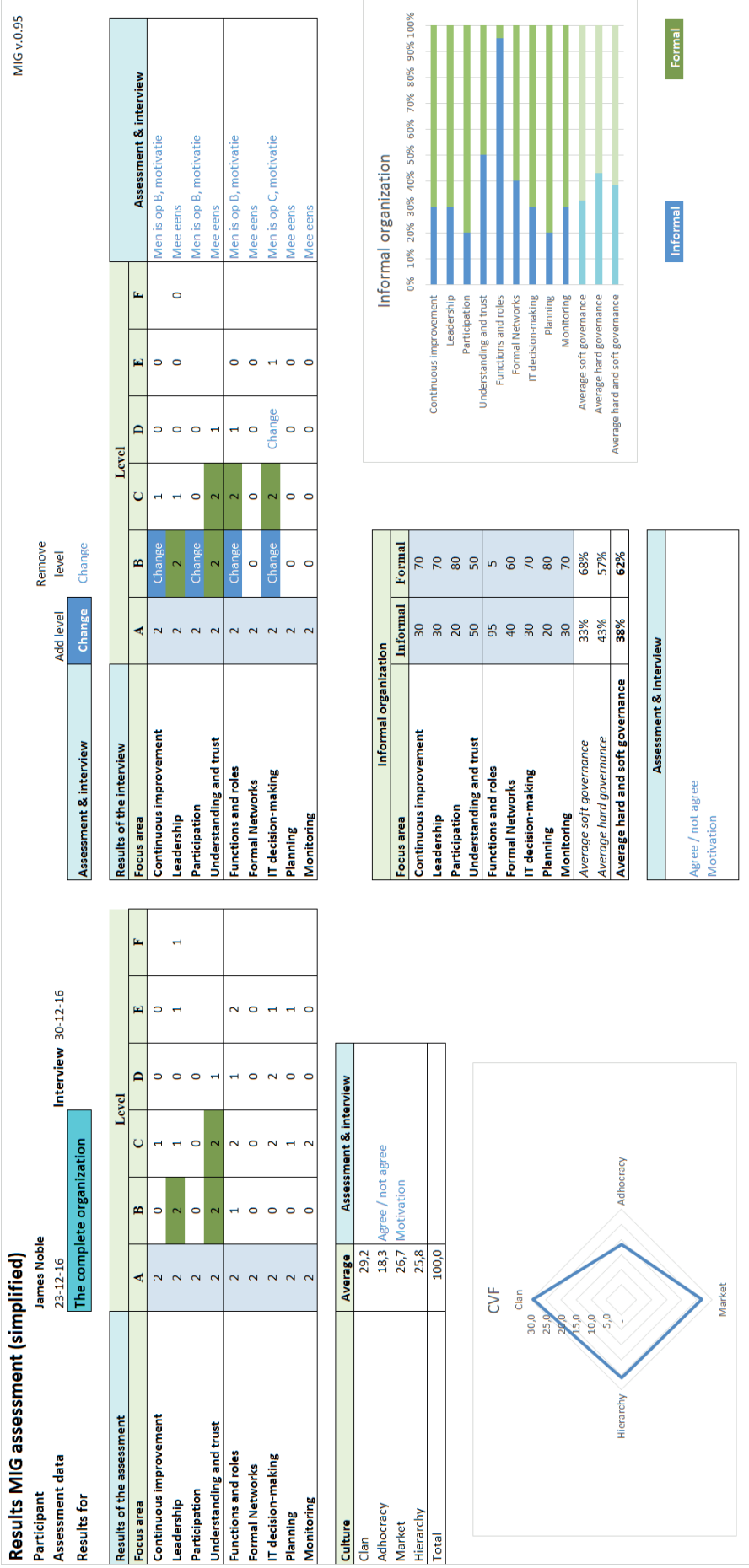

: 
The results sheet might appear more complex than the reality:

- $\quad$ The two upper tables show the results of the maturity part of the MIG model following the survey (left) and the interview (right). The tables show the maturity level reached for each focus area of the MIG model (questionnaire 1). Column A is the starting point. A coloured box means that a level has been reached. The text "Change" means that the level was changed at the request of the interviewee.

- $\quad$ The graph and table on the lower left show the results of the OCAI (questionnaire 3), consisting of the Competing Values Framework in the form of a graph and the associated data.

- $\quad$ The graph and table on the lower right show the results of the points assigned to the "informal organisation" for each focus area in the form of a graph and the associated data (questionnaire 2).

The complete questionnaires and both results sheets of the MIG instrument version 3 are included in Appendix C.

\subsubsection{Results}

We conducted 10 case studies in 2017. All studies were conducted in organisations larger than 1000 FTE. Eight were conducted by groups of four or five full-time students, and two case studies were conducted by the researchers (cases \#1 and \#2). The first and second will be described in detail in this section. 


\begin{tabular}{|c|l|c|c|c|l|l|l|}
\hline \# & Sector & \#P & \multicolumn{2}{|c|}{$\begin{array}{l}\text { Informal } \\
(\mathbf{a v g} ; \boldsymbol{)}\end{array}$} & $\begin{array}{l}\text { Culture } \\
\left(\mathbf{1}^{\text {st }}\right)\end{array}$ & $\begin{array}{l}\text { Culture } \\
\left(\mathbf{2}^{\text {nd }}\right)\end{array}$ & $\begin{array}{l}\text { Size } \\
\left(\mathbf{x 1 0 0 0} \mathbf{1}^{\mathbf{1 2}}\right.\end{array}$ \\
\hline 1 & $\begin{array}{l}\text { O; Public administration and defence; } \\
\text { compulsory social security }\end{array}$ & 10 & $58 \%$ & $11 \%$ & Hierarchy & Clan & 57 \\
\hline 2 & $\begin{array}{l}\text { O; Public administration and defence; } \\
\text { compulsory social security }\end{array}$ & 7 & $38 \%$ & $14 \%$ & Hierarchy & Clan & 110 \\
\hline 3 & $\begin{array}{l}\text { N; Administrative and support service } \\
\text { activities }\end{array}$ & 4 & $48 \%$ & $9 \%$ & Market & Hierarchy & 5.5 \\
\hline 4 & P; Education & 5 & $53 \%$ & $9 \%$ & Hierarchy & Clan & 2.9 \\
\hline 5 & K; Financial and insurance activities & 4 & $51 \%$ & $14 \%$ & Market & Hierarchy & 54 \\
\hline 6 & $\begin{array}{l}\text { M; Professional, scientific and } \\
\text { technical activities }\end{array}$ & 8 & $60 \%$ & $13 \%$ & Clan & Adhocracy & 15 \\
\hline 7 & $\begin{array}{l}\text { D; Electricity, gas, steam and air } \\
\text { conditioning supply }\end{array}$ & 5 & $46 \%$ & $10 \%$ & Clan & Hierarchy & 64 \\
\hline 8 & $\begin{array}{l}\text { I; Accommodation and food service } \\
\text { activities }\end{array}$ & 5 & $61 \%$ & $13 \%$ & Clan & Adhocracy & 0.8 \\
\hline 9 & J; Information and communication & 5 & $61 \%$ & $11 \%$ & Clan & Adhocracy & 24 \\
\hline 10 & $\begin{array}{l}\text { N; Administrative and support service } \\
\text { activities }\end{array}$ & 7 & $63 \%$ & $14 \%$ & Clan & Market & 13 \\
\hline
\end{tabular}

Table 62 Overview of case studies in 2017 (after the interviews)

\# $\quad$ is the number of the case study in 2017.

Sector is the sector of the organisation, according to NACE v. 2 (section; description).

\#P is the number of participants in the case study.

Informal represents the average and standard deviation of the percentage informal governance between the answers of the participants.

Culture shows the most dominant cultural perspective (1st = highest value) and second most dominant (2nd).

Size is the number of employees in full-time equivalents (FTE).

Appendix F.1 contains a complete overview of the end results of the case studies conducted in 2015.

\section{Detailed description of case \#1: National government}

This case study was conducted at a large independent administrative party of the Dutch government. A selection of 10 participants from business and IT were invited to participate in the case study. The participants were chosen in close collaboration with the responsible manager of one value chain of the organisation. All participants had a management position (nine) or

12 An estimation of the corporation's size is based on the 2016 annual report. 
a key role (one) in the value chain, and were involved in strategic business and IT discussions with respect to the value chain.

Table 63 shows the results before and after the interviews for the department view.

\begin{tabular}{|c|c|c|c|c|c|c|c|}
\hline \multicolumn{2}{|c|}{ Governance/focus area } & A & B & $\mathrm{C}$ & D & $\mathbf{E}$ & $\mathbf{F}$ \\
\hline \multirow{4}{*}{ 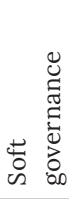 } & Continuous improvement & $10 ; 7$ & $0 ; 2$ & $0 ; 1$ & & & \\
\hline & Leadership & $6 ; 2$ & $2 ; 4$ & $2 ; 2$ & $0 ; 2$ & & \\
\hline & Participation & $10 ; 2$ & $0 ; 5$ & $0 ; 3$ & & & \\
\hline & Understanding and trust & $10 ; 6$ & $0 ; 4$ & & & & \\
\hline \multirow{5}{*}{ 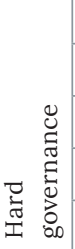 } & Functions and roles & $7 ; 4$ & $1 ; 1$ & $2 ; 5$ & & & \\
\hline & Formal networks & $8 ; 6$ & & $1 ; 3$ & $0 ; 1$ & & \\
\hline & IT decision-making & $9 ; 8$ & $0 ; 1$ & $1 ; 1$ & & & \\
\hline & Planning & $7 ; 4$ & $1 ; 2$ & $1 ; 3$ & & $0 ; 1$ & \\
\hline & Monitoring & $7 ; 3$ & $0 ; 3$ & $1 ; 3$ & & $2 ; 1$ & \\
\hline
\end{tabular}

Table 63 Results of the hard and soft governance, department view (before; after)

In general, there were considerable differences in the results (see Table 3), as demonstrated by the assessment and opinion of the participant regarding the soft governance part and relatively low number of changes to the hard governance part. Where participants suggested changes, they were always towards a higher maturity level in this case study. The participants thus always desired a change to a higher and never to a lower maturity level, compared to the maturity level displayed on the results sheet.

The rationale behind the changes provides some idea of the ways in which participants interpreted the focus areas. Some participants changed their opinion after an additional explanation of the focus areas, partly accounting for the changes.

Table 64 shows the results after the interviews for the focus areas of the context.

\begin{tabular}{|c|c|c|c|c|c|c|c|}
\hline \multicolumn{2}{|c|}{ Governance/focus area } & Min. & Max. & Avg. & $\sigma$ & Agree & Not agree \\
\hline \multirow{6}{*}{ 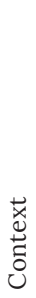 } & \multicolumn{7}{|l|}{ Culture } \\
\hline & - Clan & 25.0 & 48.0 & 35.8 & 7.3 & 10 & 0 \\
\hline & - Adhocracy & 0.0 & 25.0 & 13.5 & 9.2 & 10 & 0 \\
\hline & - Market & 0.0 & 24.2 & 13.4 & 8.9 & 10 & 0 \\
\hline & - Hierarchy & 18.3 & 68.3 & 37.3 & 17.1 & 9 & 1 \\
\hline & Informal organisation & $31 \%$ & $57 \%$ & $46 \%$ & $9 \%$ & 10 & 0 \\
\hline
\end{tabular}

Table 64 Results of the context, view: value chain, after the interview 
In the table, Min, Max, Avg. and s are the minimum, maximum, average and standard deviation of the values/percentages between the participants' answers, respectively. "Agree" and "Not agree" highlight whether the participants agreed with the result of the assessment.

The participants mostly agreed with the results. There was one exception: a participant who responded that his score for "Hierarchy" was too low (18.3) and "Market" too high (24.2).

\section{Detailed description of case \#2: National Government}

This case study was conducted at a central department of a large ministry in the Netherlands. The central department is located in The Hague and has many other branches throughout the country. Seven participants were invited to participate in this case study.

All participants were working in the same central department and were involved in strategic business and IT discussions with respect to the entire organisation. Three also had a management role.

\begin{tabular}{|c|c|c|c|c|c|c|c|}
\hline \multicolumn{2}{|c|}{ Governance/focus area } & A & B & $\mathrm{C}$ & D & $\mathbf{E}$ & $\mathbf{F}$ \\
\hline \multirow{4}{*}{ 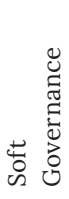 } & Continuous improvement & $7 ; 6$ & $0 ; 1$ & & & & \\
\hline & Leadership & $4 ; 1$ & $3 ; 3$ & $0 ; 2$ & & & $0 ; 1$ \\
\hline & Participation & $7 ; 5$ & $0 ; 2$ & & & & \\
\hline & Understanding and trust & $6 ; 4$ & $1 ; 2$ & $0 ; 1$ & & & \\
\hline \multirow{5}{*}{ 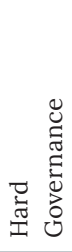 } & Functions and roles & $4 ; 3$ & $1 ; 2$ & $1 ; 1$ & $1 ; 1$ & & \\
\hline & Formal networks & $5 ; 1$ & $1 ; 3$ & $1 ; 3$ & & & \\
\hline & IT decision-making & $7 ; 4$ & $0 ; 1$ & $0 ; 2$ & & & \\
\hline & Planning & $5 ; 2$ & $2 ; 4$ & $0 ; 1$ & & & \\
\hline & Monitoring & $5 ; 3$ & $2 ; 2$ & $0 ; 2$ & & & \\
\hline
\end{tabular}

Table 65 Results of the hard and soft governance, corporate view (before; after)

In general, there were considerable variations in the results (see Table 65), as demonstrated by the assessments and opinions of the participants for the soft governance part compared to fewer variations for the hard governance part. The motivation for the changes provides some idea of the ways in which participants interpreted the focus areas. Some participants changed their opinion after an additional explanation of the focus areas, partly accounting for the changes.

Table 66 displays the results regarding the focus areas of the context in the corporate view. The context did not show different values before and after the interview. 
Results from three cycles of case studies

\begin{tabular}{|c|c|c|c|c|c|c|c|}
\hline \multicolumn{2}{|c|}{ Governance/focus area } & Min. & Max. & Avg. & $\sigma$ & Agree & Not agree \\
\hline \multirow{6}{*}{ 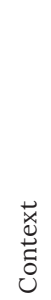 } & \multicolumn{7}{|l|}{ Culture } \\
\hline & - Clan & 12.2 & 32.5 & 23.4 & 7.5 & 6 & 1 \\
\hline & - Adhocracy & 9.2 & 24.2 & 16.0 & 4.8 & 6 & 1 \\
\hline & - Market & 5.0 & 37.5 & 17.2 & 11.0 & 5 & 2 \\
\hline & - Hierarchy & 26.7 & 61.7 & 43.4 & 12.1 & 7 & 0 \\
\hline & Informal organisation & $23 \%$ & $60 \%$ & $38 \%$ & $14 \%$ & 7 & 0 \\
\hline
\end{tabular}

Table 66 Results of the context, corporate view after the interview

In the table, Min, Max, Avg. and s are the minimum, maximum, average and standard deviation of the values/percentages between the participants' answers, respectively. "Agree" and "Not agree" illustrate whether the participants agreed with the result of the assessment.

For (organisational) "culture" and "informal organisation", the participants largely agreed with the results. Regarding "culture", in one of the interviews the participant considered the value for market excessively high. A further participant thought that the values for "Clan" and "Adhocracy" were too low and "Market" too high.

\section{Discussion of student case studies \#3 - 10 in 2017}

We conducted 10 case studies, of which two were described in detail in the previous section. In this section we describe the analysis of the student case studies. The purpose of this analysis is to determine whether the student cases demonstrate similar results. Another purpose of the analysis is to collect evaluation comments to improve the instrument (see the subsequent section).

An analysis of the remaining 43 assessments $(60-17=43)$ indicates a comparable pattern to cases one and two (see Appendix F.3).

Akin to the results in 2015 and 2016, with the exception of a few focus areas, the resulting maturity levels differed substantially (see Appendix F.3). Again, the focus area in question varied among the case studies. Thus, as in the first and second case studies, the results for each participant in the focus area maturity part of the model were very diverse.

The results of the assessment for the context were nearly always supported by the participants: 57 out of 60 participants agreed with the results of "Organisational culture" (95\%) and all agreed with the percentage for "Informal organisation" (100\%).

Therefore, again the differences in context were less pronounced (see also Table 62 and Appendix F.3). 
The case studies revealed that the "Informal organisation" is relevant in all organisations, with the average for these ten cases being $54 \%$ (std. 8\%).

\subsubsection{Evaluation of the instrument}

In general, the comments regarding the use of the MIG instrument were positive: "The way of visualising the results is very clear"; "The tool delivers very quickly an indicative impression of the maturity of several ITG processes". However, the comments on the statements and documentation were more critical: "Without the interview, the participant might misinterpret questions"; "To get reliable results, it is necessary to interview the participants".

\begin{tabular}{|c|l|c|c|l|}
\hline \# & Sector & \#Participants & \#Students & $\begin{array}{l}\text { Improvements } \\
\text { suggested: }\end{array}$ \\
\hline 1 & $\begin{array}{l}\text { O Public administration and defence; } \\
\text { compulsory social security }\end{array}$ & 10 & $\begin{array}{c}0 \\
\text { (researchers) }\end{array}$ & $2,3,5$ \\
\hline 2 & $\begin{array}{l}\text { O Public administration and defence; } \\
\text { compulsory social security }\end{array}$ & 7 & 0 & $2,3,5$ \\
\hline 3 & $\begin{array}{l}\text { N Administrative and support service } \\
\text { activities }\end{array}$ & 4 & 5 & 3,4 \\
\hline 4 & $\begin{array}{l}\text { P Education } \\
\text { (researchers) }\end{array}$ & 5 & 3,4 \\
\hline 5 & K Financial and insurance activities & 4 & 5 & 3,4 \\
\hline 6 & $\begin{array}{l}\text { M Professional, scientific and } \\
\text { technical activities }\end{array}$ & 8 & 5 & 1,2 \\
\hline 7 & $\begin{array}{l}\text { D Electricity, gas, steam and air } \\
\text { conditioning supply }\end{array}$ & 5 & 5 & 1,2 \\
\hline 8 & $\begin{array}{l}\text { I Accommodation and food service } \\
\text { activities }\end{array}$ & 5 & 5 & 2 \\
\hline 9 & $\begin{array}{l}\text { J Information and communication } \\
\text { N Administrative and support service }\end{array}$ & 5 & 5 & 5 \\
\hline
\end{tabular}

Table 67 Overview of case studies (after the interview)

\#

Sector

\#Participants

\#Students

Improvements is the number of the case study in 2017.

of the organisation, according to NACE v. 2 . is the number of participants in the case study. is the number of students conducting the case study.

are the top 5 suggested improvements for the MIG assessment:

1. The number of assessments is too low.

2. Equivocal, unclear or excessively black and white statements/questions.

3. Participants need further or better explanations of the semantics/terms used in the assessment.

4. Students would welcome a more detailed or standardised interview plan.

5. Basing the results simply on whether a participant agrees $80-100 \%$ is insufficient. 
The case studies resulted in a long list of proposed improvements for the instrument, some important, some minor. These suggested improvements were analysed and categorised. The top five most suggested improvements are included in Table 67.

\subsubsection{Evaluation of the changes in 2017}

The case studies of 2017 resulted in a list of suggested improvements (see the following subsection). However, first we discuss the results of the changes applied in 2017.

\begin{tabular}{|c|c|c|c|c|}
\hline Changes in 2017 & Discussion of the change & Improved? & Critique & Keep \\
\hline $\begin{array}{l}\text { 1. Most of the } \\
\text { questions are } \\
\text { difficult to answer } \\
\text { using just a } \\
\text { "Yes"/"No" answer. }\end{array}$ & $\begin{array}{l}\text { After changing the Yes/No scale into a } \\
\text { six-point Likert, we did not receive any } \\
\text { further comments. The only remark } \\
\text { was that it would be preferable to have } \\
\text { an option such as "Don't know". } \\
\text { We did not add this option given the } \\
\text { possibility of skipping questions and } \\
\text { adding remarks. }\end{array}$ & $\begin{array}{l}\text { Yes, fully } \\
\text { resolved. }\end{array}$ & $\begin{array}{l}\text { We received } \\
\text { no critique } \\
\text { regarding } \\
\text { the change. }\end{array}$ & Yes \\
\hline $\begin{array}{l}\text { 2. It is not clear to } \\
\text { which organisational } \\
\text { entity the question } \\
\text { refers. An option } \\
\text { would be to discern } \\
\text { two views, e.g. a } \\
\text { single department } \\
\text { and the entire } \\
\text { organisation. }\end{array}$ & $\begin{array}{l}\text { We added a definition of the views in } \\
\text { the documentation. In some of the case } \\
\text { studies, participants did not consider } \\
\text { this sufficient. } \\
\text { The description in the documentation } \\
\text { might be retained but additional } \\
\text { communication is required to } \\
\text { clarify the part of the organisation } \\
\text { emphasised for the departmental view } \\
\text { and corporate view. }\end{array}$ & $\begin{array}{l}\text { Yes, fully } \\
\text { resolved. }\end{array}$ & $\begin{array}{l}\text { We received } \\
\text { no critique } \\
\text { regarding } \\
\text { the change. }\end{array}$ & Yes \\
\hline $\begin{array}{l}\text { 4. Some questions are } \\
\text { too complex or } \\
\text { unclear. }\end{array}$ & $\begin{array}{l}\text { We supplemented the documentation } \\
\text { but received the same comments on } \\
\text { the questionnaire as in the second } \\
\text { cycle. Some questions continued to be } \\
\text { considered too unclear or general. } \\
\text { Additional improvements to the } \\
\text { documentation are thus required. An } \\
\text { alternative option might be to alter the } \\
\text { process by organising a kickoff meeting } \\
\text { with the participants of the study to } \\
\text { explain the research and terminology } \\
\text { used. In practice, organising a meeting } \\
\text { attended by all participants is very } \\
\text { difficult and time-consuming. }\end{array}$ & $\begin{array}{l}\text { Yes, fully } \\
\text { resolved. }\end{array}$ & $\begin{array}{l}\text { We received } \\
\text { no critique } \\
\text { regarding } \\
\text { the change. }\end{array}$ & Yes \\
\hline
\end{tabular}

Table 68 Evaluation of the changes in 2017

Improved Related comments decrease or stop completely

Critique Critique regarding the change?

Keep Keep the change? 
During the third cycle we used the same criteria as in the previous cycle (see Section 7.4.2). Based on the feedback of the participants and the users of the instrument, we concluded that each change may be considered an improvement. Given that the evaluation of each amendment was positive (column Keep in Table 68), there was no reason to reverse any of the changes.

Based on the feedback of the participants and the users of the instrument (the students), we can conclude that each change may be deemed an improvement.

\subsubsection{Evaluation of the results and preparation of the next cycle}

Having completed the case study, the participants of case studies \#1 and \#2 as well as the students were invited to fill out a short evaluation questionnaire. The questionnaire used a sixpoint Likert scale ranging from "Disagree completely" (valued as one point) to "Agree completely" (valued as six points).

The questionnaire was returned by eight participants in case \#1(80\%) and six participants in case \#2 (86\%), as well as 20 students (56\%).

\begin{tabular}{|c|c|c|c|c|}
\hline$\#^{13}$ & Statement & Case 1 & Case 2 & Students \\
\hline 10 & The MIG instrument is useful for conducting assessments & $4.3(0.8)$ & $4.7(0.8)$ & $4.4(1.1)$ \\
\hline 11 & $\begin{array}{l}\text { The MIG instrument is useful for practice in my } \\
\text { organisation }\end{array}$ & $4.3(1.0)$ & $4.5(0.8)$ & $3.9(1.0)$ \\
\hline 12 & $\begin{array}{l}\text { The MIG instrument combined with interviews is useful } \\
\text { for practice in my organisation }\end{array}$ & $4.9(0.9)$ & $5.2(1.0)$ & $5.1(0.9)$ \\
\hline 17 & $\begin{array}{l}\text { The results of the MIG instrument can be used in practice } \\
\text { in my organisation }\end{array}$ & 3.9 (1.1) & $4.5(1.0)$ & $4.0(1.1)$ \\
\hline 22 & $\begin{array}{l}\text { The results of the MIG instrument combined with } \\
\text { interviews can be used in practice in my organisation }\end{array}$ & $4.6(0.5)$ & $5.2(1.0)$ & $5.0(0.9)$ \\
\hline 33 & $\begin{array}{l}\text { Would you suggest any updates or improvements related } \\
\text { to the MIG instrument? }\end{array}$ & $\mathrm{N}(7) ; \mathrm{Y}(1)$ & $\mathrm{N}(6) ; \mathrm{Y}(0)$ & $\mathrm{N}(2) ; \mathrm{Y}(18)$ \\
\hline 37 & $\begin{array}{l}\text { Would you suggest any other updates or improvements } \\
\text { related to the MIG model? }\end{array}$ & $\mathrm{N}(7) ; \mathrm{Y}(1)$ & $\mathrm{N}(6) ; \mathrm{Y}(0)$ & $\mathrm{N}(15) ; \mathrm{Y}(6)$ \\
\hline
\end{tabular}

Table 69 Summary of the evaluation

The results for cases \#1 and \#2 in Table 4 are based on evaluations by the participants. The final column shows the results of the evaluation by the students. The comments for each case study have already been summarised in Table 3 . The number in parentheses is the standard deviation.

13 \# is the number of the statement on the evaluation form 
The participants and the students were generally rather positive about the usefulness and usability of the results of the instrument (in most cases being between 4 and 5 on a scale out of 6). Furthermore, the evaluation scores indicate that combining the instrument with interviews consistently results in higher scores.

In general, the responses from the students during the practical examinations were positive, such as it was a "great learning experience for our team" (case \#3).

\subsubsection{Practitioners' evaluation of the result-report (with KNVI)}

As the penultimate step of the case study protocol, a report summarising the results of the study was written, presented and discussed with the client and participants. The format of the report was created over several case studies via consultation with the clients. The report of the first case study of 2017 was anonymously shared in an evaluation Delphi session with practitioners.

This Delphi session was conducted on 19 September 2017. For this session, members of the KNVI (the largest Dutch association of IT professionals) with experience with ITG were invited to participate. As in earlier Delphi sessions, we used the innovative tool Spilter by Canast, which is a user-friendly, web-based GDSS (Spilter, 2014).

The number of participants in the session was 14. The average age of the participants was 51 years. The group consisted of two women and $12 \mathrm{men}$. All participants had a Master's degree (or equivalent).

Specific ITG training was attended by six participants. The participants all had roles in IT development or information management or were working as IT architects. Two of the participants were retired IT practitioners.

All participants had specific interest in and experience of using ITG. The average work experience was 30 years, 12 years of which with ITG. The self-reported expertise in/knowledge of ITG was 4.4 on a scale of 1 to 7 , with 4 being the average.

The discussed version of the MIG report v. 2 consisted of four parts:

I. Introduction;

II. Summary, conclusion and recommendation;

III. Results;

IV. Appendices.

A more detailed overview of the structure of the MIG report v. 2 is included in Appendix D. 
Each part was discussed separately during the Delphi session, and finally the full report was evaluated (see Table 70).

\begin{tabular}{|l|c|c|c|c|c|c|}
\hline & $\begin{array}{c}\mathbf{1} \\
\text { Pisagree } \\
\text { Part }\end{array}$ & $\begin{array}{c}\mathbf{2} \\
\text { Disagree } \\
\text { very much }\end{array}$ & $\begin{array}{c}\mathbf{3} \\
\text { Somewhat } \\
\text { disagree }\end{array}$ & $\begin{array}{c}\mathbf{4} \\
\text { Somewhat } \\
\text { agree }\end{array}$ & $\begin{array}{c}\mathbf{5} \\
\text { Agree } \\
\text { very much }\end{array}$ & $\begin{array}{c}\mathbf{6} \\
\text { Agree } \\
\text { completely }\end{array}$ \\
\hline $\begin{array}{l}\text { The MIG rapport is } \\
\text { understandable. }\end{array}$ & $0-0.0 \%$ & $0-0.0 \%$ & $5-35.7 \%$ & $5-35.7 \%$ & $4-28.6 \%$ & $0-0.0 \%$ \\
\hline $\begin{array}{l}\text { The MIG rapport is } \\
\text { useful. }\end{array}$ & $0-0.0 \%$ & $0-0.0 \%$ & $5-35.7 \%$ & $7-50.0 \%$ & $1-7.1 \%$ & $1-7.1 \%$ \\
\hline $\begin{array}{l}\text { The MIG rapport is } \\
\text { executable. }\end{array}$ & $0-0.0 \%$ & $2-14.3 \%$ & $9-64.3 \%$ & $3-21.4 \%$ & $0-0.0 \%$ & $0-0.0 \%$ \\
\hline
\end{tabular}

Table 70 Summary of the KNVI evaluation (complete report)

As can be seen in the summary, there was substantial disagreement regarding the understandability, usefulness and executability of the report. Some were very positive whereas others were very negative, and the largest group was somewhere in the middle.

We collected numerous suggestions to improve the report.

Some examples of feedback on the complete report included:

- $\quad$ "The introduction could be shorter";

- $\quad$ "Add a description of the reasons for the research";

- $\quad$ "Start with the recommendations and add substantiated arguments afterwards";

- $\quad$ "The tables need substantial explanation to be understandable";

- "Use fewer abbreviations";

- $\quad$ "If I had to decide, I would like to know which opinions are from management and which are from the workplace";

- _ " "Culture" and "Informal organisation" dominate too much in the tables";

- $\quad$ "Add a description of how the participants were selected";

- $\quad$ "Looking at the initial goal of creating a simple tool to determine how to improve ITG that includes the soft side, I think it is a fine tool".

This feedback will be used to improve the report in the next year.

\subsubsection{Conclusion}

This evaluation study intended to evaluate whether the MIG assessment instrument can be used to determine the current hard and soft ITG of an organisation according to the MIG model. This section summarises the answer to two research questions. 
SQ2.4: How can the current hard and soft ITG be measured in an organisation?

The purpose of this thesis is to evaluate how the MIG assessment instrument designed can be used to determine the current ITG maturity of an organisation according to the MIG model. This case study shows that investigators (full-time students, part-time experienced students and researchers) in each of the 10 case studies succeeded in determining the current ITG maturity of an organisation according to the MIG model in the opinions of the participants. This was reached following the case study protocol in which the assessment was combined with a structured interview.

The first case was conducted by the researchers and revealed a considerable difference between the assessment instrument and the opinions of the participants for the maturity part of the model. This was mentioned in a small part of the case studies conducted by the students, specifically cases \#1 and \#8. Based on all comments on the questionnaire of the MIG assessment instrument, a higher percentage would be expected. The reason for this might be that the participants in the first case study were asked explicitly if they agreed with the results of the assessment.

Almost all participants agreed with the results for "Culture" (97\%) and "Informal organisation" (100\%). This was no surprise for (organisational) "culture" because the MIG instrument makes use of the questionnaire of an already validated research instrument (the OCAI instrument).

We conclude that the combination of the MIG assessment instrument and structured interviews can be used to measure current hard and soft ITG. The corrected results sheet after the interview helped create a usable perspective (the corrected results sheet after the structured interviews) regarding current hard and soft ITG.

All but one of the 42 participants believed that the informal organisation represents an important part of ITG, with an average of $47 \%$ (std. 16\%). This confirms the assumption in the MIG model that "Informal organisation" must be deemed a situational part of ITG.

\section{SQ3.2: How usable is the instrument for measuring current hard and soft IT governance?}

The results sheet of the MIG assessment instrument was helpful during the structured interviews in discussing the focus areas. We received many positive comments on the usability of the tool in general: "The way of visualising the results is very clear" and it "delivers very quickly an indicative impression of the maturity of several ITG processes". The case studies additionally resulted in a long list of proposed improvements to the instrument. 
Only two of the 10 case studies stimulated comments regarding the representation of the opinions of the participants in the results. Based on all comments concerning univocal statements, a higher percentage might be expected.

An evaluation survey among participants and students yielded positive results regarding the usefulness and usability of the results of the instrument (in most cases being between 4 and 5 on a scale of 6 , see Table 69). Furthermore, the evaluation scores demonstrated that combining the instrument with interviews resulted in even higher scores (around 5).

The results of the evaluation when asked to evaluate "the usability of the results of the MIG instrument in practice in my organisation combined with interviews" resulted in scores between 4.6 and 5.2 on a scale out of 6 . This accorded with the comments received during interviews with participants in the case studies conducted between 2015 and 2017. We thus conclude that the instrument is usable in practice for measuring hard and soft ITG.

The interviews delivered valuable information regarding the reasons why the participants agreed or not with the resulting maturity levels, the graph of the Competing Values Framework, and the percentage for "Informal organisation". This information can be used to improve the instrument. All comments and suggestions for improvements have been categorised and analysed.

The top five most important improvements required were: unclear statements/questions, the number of assessments is too low, the assessment result of the maturity part is not in line with the opinions of the participants, "yes" or "no" as possible answers is insufficient, and completing the questionnaire is very time-consuming.

Substantial improvements to the instrument were applied during the design cycles. The MIG instrument v. 3 is usable in practice, but further improvements are required to reduce the deviation between the results of the instrument and the opinions of the participants, as well as to fix certain deficiencies.

\subsection{Summary of this section}

This section has provided a detailed description of the case studies conducted between 2015 and 2017. In this period, three versions of the MIG assessment instrument were used. At the beginning of the second and third cycles, the results were evaluated and used to improve the assessment instrument. 
The changes to the instrument were described in Section 6. The present section contains an overview of the results of the case studies, the evaluations and the motivations behind the changes.

This section has demonstrated that the MIG assessment instrument is usable for measuring hard and soft ITG, and when combined with interviews, the results of the instrument are usable in practice.

Substantial improvements to the instrument were applied during the design cycles. Further improvements are required to reduce the deviation between the results of the instrument and the opinions of the participants, as well as to fix certain deficiencies.

ITG can be considered an element of corporate governance (see Section 1.3.2). The following section describes the link between corporate governance and ITG. 


\section{Section 8}

\section{The link between corporate governance and IT governance}

\subsection{Introduction}

Step IV covers the use, improvement and validation of the MIG assessment instrument. An overview of the activities and results discussed in this section is shown in the research outline and marked in light blue in Figure 25.

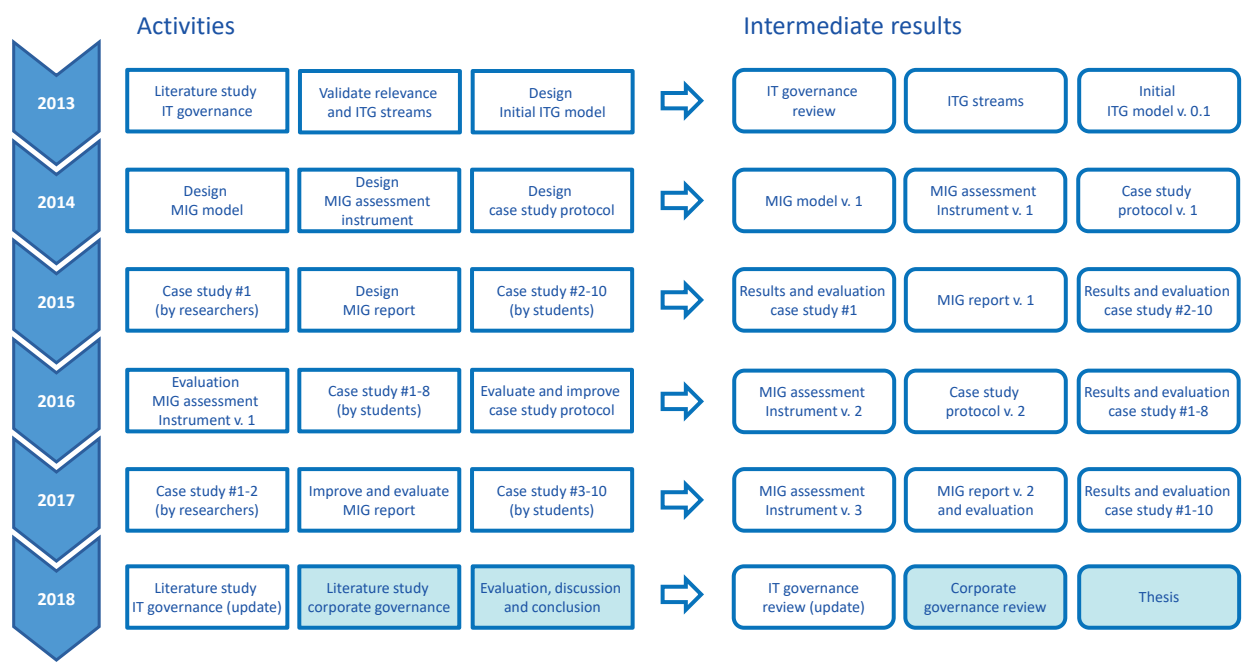

Figure 25 Activities and results of Step IV, Part B.

The research method for these activities has been described in Section 2.2. Further details regarding this specific study and the results are described in the present section.

In the previous section, we described the results of the case studies. In this section we describe the results of the following two activities:

- $\quad$ A systematic literature review on corporate governance conducted in 2018;

- $\quad$ An evaluation of the usability of the adjusted governance assessment instrument (meaning v. 3) for measuring corporate governance in an organisation. 
Section 8

The content of this section is partly based on previously published work..$^{14}$

\subsubsection{The relationship between ITG and corporate governance}

In Section 3.4.1 we described a systematic ITG literature review to determine which stream(s) of ITG align with current practices. These streams pertain either to the conformance perspective (internal and external rules and regulations) or the performance perspective (value creation; output or efficiency of business processes), although some streams relate to both perspectives.

We found six streams of ITG: four ITG streams differ in scope: IT audit, decision-making, "part of corporate governance, conformance perspective" and "part of corporate governance, performance perspective", while two streams differ in the direction in which ITG works (topdown and bottom-up) (see section 3.4.1 and Table 12).

Given that our research focuses on performance rather than conformance, we are interested in the ITG streams that pertain to the performance perspective. As IT audit largely focuses on conformance from a performance perspective, four streams remain:

- Decision-making;

- $\quad$ Part of corporate governance, performance perspective;

- Top-down;

- Bottom-up.

This section describes a systematic literature review on corporate governance with the purpose of determining whether the focus areas of the MIG model are also relevant for corporate governance.

It is known that corporate governance and ITG are related. Research has demonstrated that, for instance, organisations that have centralised their corporate governance also tend to centralise their ITG, whereas firms that have decentralised their corporate governance also tend to decentralise their ITG (Sambamurthy and Zmud, 1999, Brown and Magill, 1998, Tavakolian, 1989). However, little is known about how the relationship between corporate governance and ITG works. A better understanding of this relationship is thus required.

As described in Section 7.5.1, we introduced two perspectives in the third version of the MIG assessment instrument: a departmental and a corporate perspective. In the event that the

14 Smits, D. \& van Hillegersberg, J. 2018b. Hard and soft governance: The missing link between corporate and IT governance. Proceedings of the 6th International Conference on Management, Leadership and Governance (ICMLG), Bangkok, Thailand. Academic Conferences Limited, pp 421-430. 
focus areas of the MIG model are relevant for ITG and corporate governance, these focus areas can be seen as elements linking ITG and corporate governance.

Any causal relationship of how ITG promotes firm performance remains unclear (Vejseli and Rossmann, 2017), necessitating further research. In particular, little is known about the way in which this relationship works.

This relationship can be described in several ways, such as from a performance (added value) or conformance (rules and regulation) perspective. This thesis follows the stream in which ITG is considered an integral part of corporate governance, and we focus on the performance perspective. Covering corporate governance in its totality might however require additional focus areas.

This section focuses on the relationship between corporate governance and ITG, and contributes to an understanding of how ITG and corporate governance are related.

\subsection{Research method}

Our research is built on the assumption that improving ITG results in greater firm performance. Given that ITG and corporate governance are connected, we are interested in understanding how this relationship works as well as the interconnections between ITG and corporate governance. This might be helpful in understanding how to ameliorate firm performance by improving ITG.

The research process for this study was as follows:

a. Conduct a systematic literature review to locate the corporate governance literature on ITG focus areas;

b. Create an update-adjusted version of the MIG assessment instrument with a view on ITG and corporate governance (see Section 6.5);

c. Perform a series of case studies using the adjusted instrument to assess ITG and corporate governance (see Section 7.5);

d. Evaluate the results of the study.

The goal of this thesis is to answer the following two research questions:

SQ3.3: How usable are the ITG focus areas for corporate governance research? 
Section 8

SQ3.4: How usable is version 3 of the assessment instrument for measuring corporate governance in an organisation?

The results of the third cycle described in Section 7.5 consist of two parts: a systematic literature review for and a series of 10 case studies using the MIG model and the MIG assessment instrument v. 3.

The Systematic literature review

A systematic literature review (see Section 2.2.1) on corporate governance was prepared and conducted using the Scopus database.

In Scopus, we first selected papers pertaining to "corporate governance" in the title, abstract or author keywords. Within this large set of papers, we selected papers related to the focus areas and domains of ITG according to the MIG model. Furthermore, we selected papers related to "hard governance" and "soft governance" in general, as well as "soft power" given that this theory forms the basis of soft governance (Nye, 1990). A subsequent selection was used to determine the papers in scope.

To be in scope, the paper had to satisfy the following rules:

- $\quad$ The topic of the paper must be corporate governance;

- $\quad$ The topic of the keyword found must be used as defined in the MIG model;

- $\quad$ The paper must be written in English, German or Dutch;

- $\quad$ Claims must be justified or based on research;

- $\quad$ Duplicated studies are excluded.

For some of the focus areas, the result set was too large to analyse (300 papers or more). Given that the goal of the present section is to validate the existence of the focus areas in the current corporate governance literature (and we do not required a complete list of the papers), we changed our approach. For these focus areas, we only evaluated the first 100 papers sorted in descending order of number of citations, and selected between five and 10 examples that satisfied the aforementioned rules.

\subsection{Results of the systematic literature review}

A selection of papers on "corporate governance" between November and December 2017 in Scopus resulted in a set of 15,214 papers. A sub-selection was made for each focus area in the 
MIG model. In some cases, additional keywords were added to include different notations of the same notion.

\begin{tabular}{|l|c|c|l|}
\hline Documents (total \# selected =15,214) & $\#$ & $\%$ & Paper validation \\
\hline On "continuous" and "improvement" & 65 & 0.43 & Examples \\
\hline On "leadership" & 2,885 & 19 & Examples \\
\hline On "participate" or "participation" & 1,135 & 7.5 & Examples \\
\hline On "understanding" and "trust" & 633 & 4.2 & Examples \\
\hline On "functions" and "roles" & 1,604 & 11 & Examples \\
\hline On "formal" and "networks" & 321 & 2.1 & Examples \\
\hline On "IT" and "decision-making" & 1,204 & 7.9 & Examples \\
\hline On "planning" & 1,915 & 13 & Examples \\
\hline On "monitoring" & 3,956 & 26 & Examples \\
\hline On "culture" & 2,667 & 18 & Examples \\
\hline On "informal" and "organisation" & 359 & 2.4 & Examples \\
\hline On "sector" & 3,162 & 21 & Examples \\
\hline On "soft" "governance" or "hard" "governance" & $3(149)$ & 0.02 & Complete \\
\hline On "soft power" & $2(8)$ & 0.013 & Complete \\
\hline
\end{tabular}

Table 71 Documents in Scopus on corporate governance grouped by specific topics

Table 71 displays the results for each focus area or keyword. The total set of corporate governance papers was 15,214.

The first column displays the number of papers found for each keyword. The number within parentheses is the number of papers originally found in the selection. The second number shows the percentage of the total set of corporate governance papers. The final column describes the validation to the rules as described in Section 8.2.

For the remainder of the keywords, we selected examples. Aside from "continuous improvement", the examples could be found within the first 100 papers when sorted in descending order of number of citations. For all focus areas (as shown in Table 72) we were able to find between five and 10 examples of corporate governance papers that satisfy the rules. An overview of the selected papers is included in Table 72.

We validated the complete set for "hard" "governance", "soft" "governance" and "soft power". Given that the number of papers found for "soft governance" and "hard governance" was very low 
(only one), we conducted an additional selection using "soft" "governance" or "hard governance".

On this occasion we identified two additional papers, resulting in three papers in total.

\begin{tabular}{|c|c|c|c|}
\hline Focus area or topic & Initial & Selected & Selected papers \\
\hline $\begin{array}{l}\text { On "continuous" and } \\
\text { "improvement" } \\
\text { (complete list) }\end{array}$ & 9 & 9 & $\begin{array}{l}\text { (Braithwaite and Travaglia, 2008), (Conti, 2003), (Delgado } \\
\text { and Velthuis, 2015), (Fulgence, 2014), (Lasierra Esteban, } \\
\text { 2016), } \\
\text { (Li, 2017), (Salvioni et al., 2014), (Seamer, 2014), (Xie and } \\
\text { Li, 2011). }\end{array}$ \\
\hline On "leadership" & 6 & 6 & $\begin{array}{l}\text { (Cannella et al., 2009), (Daily et al., 2002), (Huse and } \\
\text { Solberg, 2006), (Huse, 2007), (Jo and Harjoto, 2011), } \\
\text { (Rechner and Dalton, 1991). }\end{array}$ \\
\hline $\begin{array}{l}\text { On "participate" or } \\
\text { "participation" }\end{array}$ & 14 & 10 & $\begin{array}{l}\text { (Braithwaite and Travaglia, 2008), (Brammer et al., 2012), } \\
\text { (Giannetti and Koskinen, 2010), (Gorton and Schmid, } \\
\text { 2004), (Ingley and Van Der Walt, 2001), (Jackson, 2005), } \\
\text { (Jackson and Apostolakou, 2010), (Jara-Bertin et al., } \\
\text { 2008), (Spitzeck and Hansen, 2010), (Westphal and } \\
\text { Khanna, 2003). }\end{array}$ \\
\hline $\begin{array}{l}\text { On "understanding" } \\
\text { and "trust" }\end{array}$ & 6 & 6 & $\begin{array}{l}\text { (Bass and Steidlmeier, 1999), (Caldwell and Karri, 2005), } \\
\text { (Hendry, 2004), (Learmount, 2004), (Sacconi, 2007), } \\
\text { (Verhezen, 2010). }\end{array}$ \\
\hline $\begin{array}{l}\text { On "IT" and "decision- } \\
\text { making" }\end{array}$ & 20 & 10 & $\begin{array}{l}\text { (Carpenter and Westphal, 2001), (Davis and Greve, 1997), } \\
\text { (Forbes and Milliken, 1999), (Gomez-Mejia et al., 2011), } \\
\text { (Graebner and Eisenhardt, 2004), (Huse, 2007), (McNulty } \\
\text { and Pettigrew, 1999), (Mustakallio et al., 2002), (Porter, } \\
\text { 1992), } \\
\text { (Tirole, 2001). }\end{array}$ \\
\hline $\begin{array}{l}\text { On "functions" and } \\
\text { "roles" }\end{array}$ & 13 & 9 & $\begin{array}{l}\text { (Abbott and Parker, 2000), (Abraham and Cox, 2007), } \\
\text { (Aguilera, 2005), (Andres and Vallelado, 2008), (Choi and } \\
\text { Wong, 2007), (Cornforth, 2004), (Guest, 2008) (Huse, } \\
\text { 2007), (Tuggle et al., 2010). }\end{array}$ \\
\hline $\begin{array}{l}\text { On "formal" and } \\
\text { "networks" }\end{array}$ & 7 & 7 & $\begin{array}{l}\text { (Bezemer et al., 2007), (Davis and Greve, 1997), } \\
\text { (Filatotchev and Nakajima, 2010), } \\
\text { (Krug and Hendrischke, 2008), (Nicholson et al., 2004), } \\
\text { (Spira, 1999), (Toms and Filatotchev, 2004). }\end{array}$ \\
\hline On "planning" & 10 & 9 & $\begin{array}{l}\text { (Bedard and Johnstone, 2004), (Carpenter et al., 2003), } \\
\text { (Cyert et al., 2002), (Gedajlovic et al., 2012), (Kim et al., } \\
\text { 2004), (Kroll et al., 2008), (Nelson, 2003), (Orhangazi, } \\
\text { 2008), (Von Solms, 2001). }\end{array}$ \\
\hline On "monitoring" & 23 & 10 & $\begin{array}{l}\text { (Abbott et al., 2004), (Carpenter and Westphal, 2001), } \\
\text { (Klein, 2002), (Linck et al., 2008), (Maug, 1998), (Peasnell } \\
\text { et al., 2005), (Rediker and Seth, 1995), (Sundaramurthy } \\
\text { and Lewis, 2003), (Tirole, 2001), (Wiseman and Gomez- } \\
\text { Mejia, 1998). }\end{array}$ \\
\hline On "culture" & 9 & 9 & $\begin{array}{l}\text { (Chin et al., 2004), (Halter et al., 2009), (Hendry, 2004), } \\
\text { (Heracleous, 2003), (Ho, 2012), (Lee, 2012), (Lefkowitz, } \\
\text { 2006), (Mohamed et al., 2012), (Verhezen, 2010). }\end{array}$ \\
\hline
\end{tabular}


The link between corporate governance and IT governance

\begin{tabular}{|l|c|c|l|}
\hline Focus area or topic & Initial & Selected & Selected papers \\
\hline $\begin{array}{l}\text { On "informal" and } \\
\text { "organisation" }\end{array}$ & 5 & 5 & $\begin{array}{l}\text { (Donnelly and Mulcahy, 2008), (Farndale et al., 2010), } \\
\text { (Filatotchev and Nakajima, 2010), (Globerman et al., 2011), } \\
\text { (Verhezen, 2010). }\end{array}$ \\
\hline On "sector" & 25 & 10 & $\begin{array}{l}\text { (Allen et al., 2005), (Aoki et al., 2007), (Brennan and } \\
\text { Solomon, 2008), (Cioffi and Höpner, 2006), (Claessens } \\
\text { and Yurtoglu, 2013), (Crane et al., 2009), (Heath and } \\
\text { Norman, 2004), (Mak and Li, 2001), (Nickell et al., 1997), } \\
\text { (Orhangazi, 2008). }\end{array}$ \\
\hline $\begin{array}{l}\text { On "soft" "governance" } \\
\text { or "hard" "governance" } \\
\text { (complete list) }\end{array}$ & 149 & 3 & $\begin{array}{l}\text { (Kudrle, 2010), (Peltokorpi and Tsuyuki, 2007), (Uldam } \\
\text { and Hansen, 2017). }\end{array}$ \\
\hline $\begin{array}{l}\text { On "soft power" } \\
\text { (complete list) }\end{array}$ & 8 & 2 & (Nwafor, 2014a), (Nwafor, 2014b). \\
\hline
\end{tabular}

Table 72 Selected corporate governance papers per focus area or topic

The column "Initial" refers to the number of papers initially found using the key in the first 100 papers when sorted in decreasing number of citations. The complete list was used for the focus area "continuous improvement" and the topics "soft governance" or "hard governance" and "soft power" because the number of papers found was less than 300 .

The column "Selected" refers to the number of papers meeting the selection criteria described in Section 8.2.

\subsection{Discussion}

The systematic literature review indicated that all 12 focus areas could be found (in the title, abstract or keywords) in a substantial proportion of the corporate governance literature. Aside from "continuous improvement", all focus areas are mentioned in $2 \%$ of the papers or more (> 300 papers). Some topics such as "monitoring" (26\%), "leadership" (19\%) and "culture" (18\%) were mentioned even more often.

In most cases, the number of selected papers was too large to analyse. For these focus areas, we collected between five and 10 examples. Finding papers satisfying the rules defined in Section 8.2 was simple within the first 100 papers when sorted in descending order of number of citations. For continuous improvement, the complete set of 65 papers has been used. These examples are sufficient for our purpose as they demonstrate the relevance of the focus areas of the MIG model for corporate governance.

Literature covering the topic of "soft governance" or "soft power" is quite rare. In the MIG model, soft governance is related to soft power. Other concepts that might be substituted for 
"hard governance" or "soft governance" may be available in corporate governance research, but we did not find any of these in the example papers collected. However, it was noted that the trichotomy of process, structure and relational mechanisms was also used in corporate governance literature (just as in ITG literature). In corporate governance literature, soft governance is sometimes related to "soft law" (as we found 123 papers).

A preliminary conclusion drawn based on the findings of the systematic literature review is that soft governance does not receive much attention in the contemporary corporate governance literature.

The high percentages for the focus areas and the low score for hard and soft governance might owe to the fact that the corporate governance literature has since the beginning of the 1990s been dominated by "a US research tradition with a focus on protecting the investors' stakes" (Huse, 2005a) or the dominant agency theory (Dalton et al., 1998).

In practice, an integrated approach for ITG and corporate governance is required because these focus areas influence and interact with one another. In recent papers, ITG is occasionally called "corporate governance of IT" (Calder, 2008) or "enterprise governance of IT" (Dietz and Hoogervorst, 2012, Van Grembergen and De Haes, 2010). Others, like the International Federation of Accountants (IFAC, 2004) define enterprise governance as consisting of "corporate governance" focusing on conformance and "business governance" focusing on performance.

Nerantzidis et al. (2012) have demonstrated that corporate governance definitions are inconsistent: some focus on the conformance perspective; others on the conformance perspective. As the term "corporate governance" is used much more extensively in literature (and practice) than "enterprise governance", we prefer the use of the term "corporate governance". We follow the stream in which ITG is seen as an integral part of corporate governance. To ensure consistency in definition, we deem the non-IT part within corporate governance "business governance".

Thus, for the relationship between corporate governance and ITG, we use the following simple model (Figure 26). 

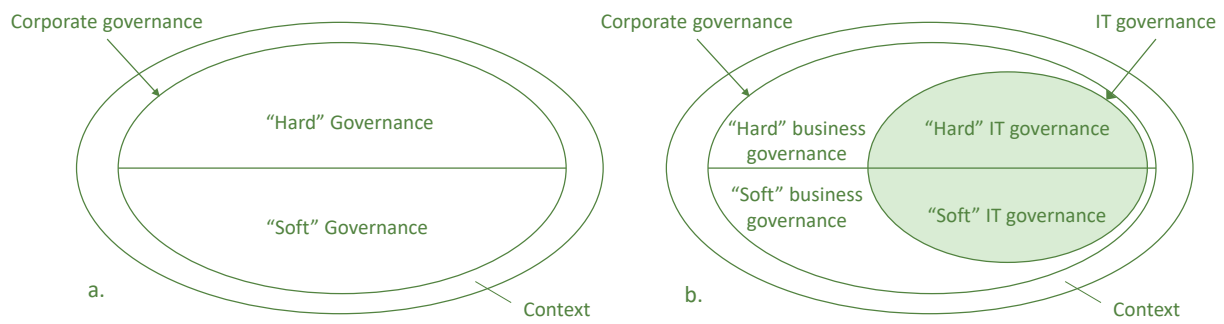

Figure 26 Corporate governance, IT governance and the context.

In Figure 26a, corporate governance is divided into three parts: hard governance, soft governance, and a context, just like the MIG model for ITG. Research on soft governance is an example of such an integrated approach.

The systematic literature review demonstrated that all focus areas are covered in the contemporary corporate governance literature. Given that corporate governance and ITG are related topics, corporate governance should attend to the same areas. In Figure 26b, ITG is presented as an integrated part of corporate governance. Figure $26 \mathrm{~b}$ also indicates that there might be more focus areas relevant for corporate governance (the part outside ITG). In this section, we focus on the 12 focus areas of the MIG model.

The case studies' results demonstrate (see Section 7.5) how the adjusted MIG assessment instrument can be used to collect data on an organisation's ITG and corporate governance. Given that organisations are very heterogeneous, it is not easy to make general conclusions based on cross-case analysis for the maturity part of the model.

Some of the data of the context are aligned with the current corporate governance literature. The contextual factors most often used in corporate governance research are, according to (Huse, 2005b), cultural differences, the industry (or sector) and firm size. Cultural differences and sector are also included in the contextual part of the MIG model, and an estimation of the firm size is included in Table 71.

The results reveal that 60 participants valued the informal organisation with 54\% (informal compared with the formal organisation) and thus at least as important as the formal organisation.

Corporate governance literature on "informal organisation" can be found from several perspectives such as strategic, standards and rules or the function of the executive (Williamson, 1999, Wieland, 2005, Barnard, 1938). Sometimes corporate governance literature on the "informal organisation" discusses other related topics like "informal meetings" or "informal social control", or an "ad hoc and informal" decision-making process. Alternatively it demonstrates "that boards 
almost always reach unanimous agreement in making major policy decisions [and] any initial reservations that directors have about a decision are typically worked out informally in advance of formal board meetings" (Westphal and Khanna, 2003, Hirsch, 1982, Adams, 2002).

Based on the case studies' results, we presume that "informal organisation" should also be a contextual factor for corporate governance.

\subsection{Conclusion}

Given that corporate governance and ITG are related topics, corporate governance should pay attention to similar areas. A broader perspective of corporate governance and a multitheoretical approach is required to determine essential focus areas to improve organisational functioning (Daily et al., 2003). The MIG model is an example of such a model. This section discusses the answers to our research questions:

\section{SQ3.3: How usable are the ITG focus areas for corporate governance research?}

The systematic literature review showed that all 12 focus areas of the MIG model are additionally covered by the corporate governance literature. This aligns with the ITG stream that defines ITG as a part of corporate governance. In practice, ITG and corporate governance must be integrated. How this relationship works, however, is unclear. This section demonstrates 12 focus areas that are relevant for ITG and corporate governance, although there may be additional relevant focus areas for corporate governance. Corporate governance literature covering hard or soft governance in the definition of "soft power" remains rare.

With the exception of "continuous improvement", the focus areas are found (in the title, abstract or keywords) in large numbers in corporate governance literature. Sharing corresponding definitions with the MIG model. Examples of corporate governance literature on "continuous improvement" have also been found but in substantially smaller numbers.

\section{SQ3.4: How usable is version 3 of the assessment instrument for measuring corporate governance in an organisation?}

During the second part of this section, we used the adjusted MIG assessment instrument to collect data on the ITG and corporate governance through 10 case studies. An analysis of the data of the context helped confirm the relevance of the contextual factors most often used in 
corporate governance research (cultural differences, the industry or sector and firm size) (Huse, 2005b), as these were all considered relevant by the participants. The results also demonstrated that 60 participants valued the informal organisation with 54\% (informal compared with the formal organisation). Indeed, in their opinions, the informal organisation is as important as the formal organisation. We presume that informal organisation should constitute a contextual factor for corporate governance too.

\subsection{Summary of this section}

Corporate governance and ITG are related. However little is known regarding the way in which this relationship works. This section has provided a detailed description of the results of a systematic literature review on corporate governance conducted in 2018.

Current corporate governance definitions are inconsistent. We follow the stream in which ITG is considered an integral part of corporate governance. To keep the definitions consistent we define the non-IT part within corporate governance as "business governance". Corporate governance can be divided into three parts: hard governance, soft governance, and a context, just like the MIG model for ITG.

The study has demonstrated that all 12 focus areas of the MIG model are additionally covered by corporate governance literature. Furthermore, it has highlighted that the 12 focus areas of the MIG model are relevant for ITG and corporate governance.

We presume that the "informal organisation" should be a contextual factor for corporate governance as well.

The following part of this thesis is the conclusion section, along with a description of limitations and future research. 


\section{Part V}

\section{Conclusions, limitations and future rese a r ch}

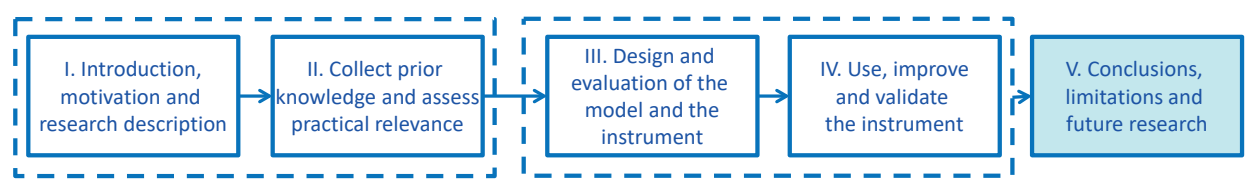

Figure 27 Thesis Part V. Conclusions, limitations and future research

\section{Section}

9. Conclusions, limitations and future research 


\section{Section 9 \\ Conclusions, limitations and future rese a r ch}

\subsection{Introduction}

The original rationale behind this research was the experience in practice that ITG represents an ongoing concern for organisations worldwide. Indeed, a common body of ITG knowledge or a widely used ITG framework appears to be lacking, and in practice organisations use various frameworks or methods for ITG.

Some studies indicate that firms with superior ITG may enjoy more than $20 \%$ greater profits than firms with poor governance, and as such, ITG is an important issue meriting improvement. How then can organisations improve their ITG? We define ITG improvement as increasing the effectiveness of ITG, leading to our main research question:

\section{[MRQ] How can the ITG of an organisation grow in maturity to become more effective?}

To answer this research question, we conducted three steps:

- $\quad$ First we compared current ITG practices and theoretical frameworks, discussed the results with practitioners and concluded that a mismatch exists.

- $\quad$ Second we determined the focus areas to be included in a framework covering ITG and the maturity model to be used so that ITG may grow. This step resulted in the design of the MIG model and the MIG assessment instrument.

- $\quad$ Third we evaluated the artefacts designed in three cycles and a few dozen case studies in order to test its usability and to evaluate whether the organisations that grow in ITG maturity (for one or more of the focus areas of the MIG model) become more effective, too.

This final section summarises the conclusions and provides answers to the research questions, the limitations of the research described in the thesis and some suggestions for future research. 
Section 9

\subsection{Conclusions}

\subsubsection{T governance streams}

The first research question concerned ITG streams and the disjuncture between theory and practice:

[RQ1] What practices constitute IT governance?

To answer this question we conducted a systematic literature review proposing an integrated view identifying six ITG-streams. In a Delphi study we asked practitioners, the CIOs of large organisations, to assess the relevance of each of these six streams. All streams were considered important, although the relative importance varied between organisations and sectors. Furthermore, we concluded that there remains a mismatch between IT governance practices and theoretical frameworks.

RQ1 was divided into two sub-questions:

\section{SQ1.1: Which stream(s) of IT governance best align with current practices?}

We found six streams of ITG:

- $\quad$ Four ITG streams differed in scope: IT audit, decision-making, "part of corporate governance, conformance perspective" and "part of corporate governance, performance perspective";

- $\quad$ Two streams differed in the direction in which ITG works: top down and bottom up (see Table 12).

In practice, specific ITG frameworks such as COBIT or ISO/IEC 38500 are only used by a small percentage of the organisations (13\% and $8 \%$, respectively). The remainder of the organisations use diverse frameworks such as service management frameworks (ITIL), security frameworks (ISO/IEC 17799, ISO/IEC 27000 or other), internally developed frameworks, quality frameworks like Six Sigma, project management frameworks like (PMI/PMBOK or PRINCE2) or otherwise (see Table 2).

Frameworks found in the literature are very different from those used in practice. An example is Novotny et al. (2012), who define ITG as having seven input and two output dimensions (see Table 3). In the literature, dimensions do not need to be operational. ITG dimensions like "Business value delivery" or "Business/IT alignment" (see Table 3) are not easy to use in practice. 
Therefore, in this thesis, we designed artefacts using more practical terms like "disciplines" or "focus areas", in which the terms selected must be usable for scientists and practitioners alike.

\section{SQ1.2: According to practitioners, which disciplines should play an important role in ITG?}

Our literature review revealed that publications covering ITG combined with portfolio management and architecture appeared to start growing from 2006/2007, although they remain rare. Case studies in the literature contain clear descriptions regarding the ways in which ITG, architecture and portfolio management relate in practice (Wittenburg et al., 2007). Papers describing best practices concerning the combination of disciplines are also available (Niemann, 2006). In the literature, architecture and portfolio management are seen as important disciplines for ITG, too. A group of CIOs agreed in a Delphi workshop on the relevance of these disciplines for ITG (see Table 17). These were mentioned by the CIOs most frequently (respectively $62 \%$ and $46 \%$ ).

When asking for indications on how to improve ITG, it is interesting to note that the resulting answers to the question "What has to be done to improve ITG?" did not refer to any particular disciplines (Table 18).

Indeed, the responses were completely different. Most answers (86\%) can be categorised as part of responsibility, performance and human behaviour. The highest score for "good ownership of processes and corresponding applications" indicates that there are still issues regarding responsibilities and accountabilities.

As one of the core issues of ITG, this seems a little contrary to the high scores for the rating of the ITG practices.

Improvements to portfolio management, architecture, security, project management, program management or innovation were not mentioned by the CIOs. Given that these disciplines also did not appear in the results from the Delphi sessions when designing the MIG instrument, we did not pursue research on disciplines.

\section{SQ1.3: Which (new) ITG maturity models are available in the literature that can be used in practice?}

The update to the systematic literature review in 2018 revealed 34 papers discussing ITG maturity since 2012. COBIT was used in 13 papers and in five others was combined with other best practice frameworks. COBIT 5.0 includes the social dimension by discerning seven enablers: 
"Principles, policies and frameworks", "Processes", "Organisational structures", "Culture, ethics and behaviour", "Information", "Services, infrastructure and applications" and "People, skills and competences". These can then be translated into four common dimensions: "Stakeholders", "Goals", "Life-cycle" and "Good practices" (Oliver and Lainhart, 2012). However, such a translation might be an oversimplification.

With one exception, none of the selected maturity model-related papers focused on social or (more specifically) the soft governance-related focus areas included in the MIG model. One exception was the research paper by Saetang and Haider (2012), who selected the Duality of Technology (Orlikowski, 1992) and the Adaptive Structuration Theory (DeSanctis and Poole, 1994) to develop a research framework to investigate effective ITG implementation. Both can be seen as alternative representations of hard and soft governance. However, the research framework does not include a maturity model. Maturity is measured by performance evaluation as the outputs of the organisational environment.

Nevertheless, when the search was widened to consider papers related to COBIT but not ITG maturity, more papers were easily found (e.g. for "culture") (Prinz, 2015).

We found two frameworks covering hard and soft ITG: in COBIT 5.0 in a holistic way and in the MIG model developed in this thesis in a more practical way. None of the selected papers demonstrated a practical means of using COBIT 5.0 to measure or improve hard and soft governance. Aside from the MIG model, social elements like collaboration, behaviour and culture are not included in these frameworks.

Finding common dimensions between theory and practice that are usable in practice represented the starting point for the design of the MIG model. This design process (for example) demonstrated the requirement for different maturity models for each soft governance focus area (Smits and van Hillegersberg, 2015).

SQ1.4: Is there (still) a mismatch between IT governance practice and theoretical frameworks?

As described in the response to SQ1.2, publications on research covering ITG combined with portfolio management and architecture are rare and a group of CIOs confirmed their relevance. We can see that there is substantial correspondence between the mentioned disciplines and the frameworks used in practice.

However, these disciplines deviate from a conventional list of dimensions (see Table 3). The literature review also delivered examples of papers in which alternative dimensions were 
suggested (Chin et al., 2004, Maidin and Arshad, 2010, Nfuka and Rusu, 2010, Mohamed et al., 2012).

The case studies we conducted demonstrate that the MIG model and the MIG assessment instrument require improvements, but in general the participants agreed that the instrument is usable when combined with interviews. The MIG model is largely based on frameworks from the appropriate literature (see Table 29).

Therefore, dimensions, disciplines and frameworks do not match (although some disciplines can be used to implement some of the dimensions). Consequently, we conclude that a disjuncture remains between ITG practice, frameworks and theory.

During the update of the systematic literature review in 2018, no new ITG maturity model was found that covers hard and soft ITG. Thus, it would appear that as of summer 2018, hard and soft ITG are covered by COBIT 5.0 in a holistic way and in the MIG model in a more practical way. Applying the MIG model is likely to help narrow the gap between ITG maturity theory and practice.

\subsubsection{Defining I Tovernance maturity}

The second research question concerned how organisations grow in ITG maturity:

[RQ2] How can the IT governance of an organisation grow in maturity?

To answer this question it was crucial to determine the focus areas of ITG. Our research was limited in scope to the performance perspective. After applying the scope to the six streams, four streams remained (Table 12). Given the "bottom-up" stream (among others), we sought soft governance focus areas to complement a mainstream list of hard governance focus areas. Thus, the MIG model and MIG assessment instrument were designed for hard and soft IT governance.

RQ2 was divided into five sub-questions:

The first three resulted in the design of the MIG model; the final two resulted in the design of the MIG assessment instrument. 
Section 9

\section{Design of the MIG model}

\section{SQ2.1: Which focus areas should an ITG maturity model for soft and hard governance contain?}

In Delphi sessions with selected practitioners (ITG experts), we defined a set of focus areas for hard and soft governance that resulted in several versions of the MIG model. This part of the research was published in 2014 and described in detail in Sections 5.3 and 5.4.

We concluded that besides hard governance, both the social aspects of governance and the context as a situational element must be included as part of the model, as supported by practice and literature.

Thus, the list of focus areas included focus areas for the context, too. The end result was a list of focus areas for hard and soft ITG (see Table 29).

\section{SQ2.2: What type of maturity model do we need?}

In a subsequent series of Delphi sessions, existing maturity models were selected to be added to the maturity part of the model. This part of the research was published in 2015 and described in detail in Section 5.5. The end result was a list of maturity models for the maturity part of the ITG model (see Table 32 and Table 33).

\section{SQ2.3: What are the capabilities of each focus area?}

During the same series of Delphi sessions we also discussed the capabilities of each focus area. The capabilities selected were the capabilities from the existing maturity models. This part of the research was also published in 2015 and described in detail in Section 5.5. The end result was a list of capabilities for the maturity part of the ITG model (see Table 38).

\section{Design of the MIG assessment instrument}

\section{SQ2.4: How can the current hard and soft IT governance be measured in an organisation?}

We opted to design a focus area maturity model for ITG because these provide greater guidance on incremental improvements than do fixed-level maturity models (see Figure 15). Furthermore, 
we made it situational by adding several focus areas for the context. This part of the research was published in 2015 and described in detail in Section 5.5.

One element that was not solved at the time of the publications in 2014 and 2015 was the model to be used for the focus area "Informal organisation" in the context. In Section 5.6 we described this update and the end result: the MIG model (Table 49).

\section{SQ2.5: How can we develop an assessment instrument based on the maturity model?}

The MIG assessment instrument was based on version 1 of the MIG model and was developed using design science. This thesis describes three design cycles between 2015 and 2017 as well as three versions of the MIG assessment instrument.

These results of the research were published in 2017 for the first time and described in detail in Section 6.

The end result comprised several versions of the MIG assessment instrument, an artefact created using Excel consisting of two parts:

1. The first part is intended for the participant and contains the statements and other questions of the assessment questionnaire;

2. The second part is for the researcher and is used to create the results sheet (see Figure 20).

\subsubsection{Measuring I governance maturity}

The third research question concerned IT governance maturity and the relationship with organisational performance:

[RQ3] How can we measure the IT governance maturity of an organisation?

To answer this question we had to determine how to use the MIG assessment instrument to assess the current ITG of an organisation, and to evaluate the usability of the MIG model and the MIG assessment instrument for determining the current ITG.

Given that ITG is an element of corporate governance, we were also interested in determining the usability of the results for determining (elements of) the current corporate governance of an organisation, as well as the changes that would improve its usability for corporate governance research. 
Section 9

RQ3 was divided into five sub-questions:

SQ3.1: How can we use the MIG assessment instrument to measure current hard and soft IT governance?

To measure current hard and soft ITG, we defined a case study protocol. This part of the research was published in 2017 and described in Section 6.2.2. Initially (that is, until the instrument has been completely validated and the difference between the results of the assessment and the interview is rendered negligible), the assessment instrument should be combined with interviews. A more detailed description of the case study protocol is included in Section 6.2.2.

During the interviews, participants were asked to respond to the results of the instrument. This created two type of results: the results of the assessment instrument and the corrected results after the interviews.

SQ3.2: How usable is the instrument for measuring current hard and soft IT governance?

This research question was discussed in detail in Section 7.5 and presented at the Centeris 2018 conference in Lisbon. The conclusion of this section is that the combination of the MIG assessment instrument and structured interviews is useful for measuring current hard and soft ITG.

\section{Relating ITG and corporate governance}

These three research questions were discussed in detail in Section 8 and presented at the 6th International Conference on Management, Leadership and Governance in Bangkok in 2018.

\section{SQ3.3: How usable are the ITG focus areas for corporate governance research?}

The systematic literature review revealed that all 12 focus areas of the MIG model are also covered by the corporate governance literature. Thus, these areas are also relevant for ITG and corporate governance. Nevertheless, there may exist additional relevant focus areas for corporate governance.

SQ3.4: How usable is version 3 of the assessment instrument for measuring corporate governance in an organisation? 
An analysis of the data of the context helped confirm the relevance of the contextual factors most often used in corporate governance research (cultural differences, the industry or sector and firm size) (Huse, 2005b), as these were all deemed relevant by the participants.

\section{SQ3.5: How do ITG, corporate governance and organisational effectiveness relate?}

In practice, an integrated approach for ITG and corporate governance is required because these focus areas influence and interact with one another. Research on soft governance is an example of such an integrated approach. For the relationship between corporate governance and ITG, we use the following simple model (see Figure 28).
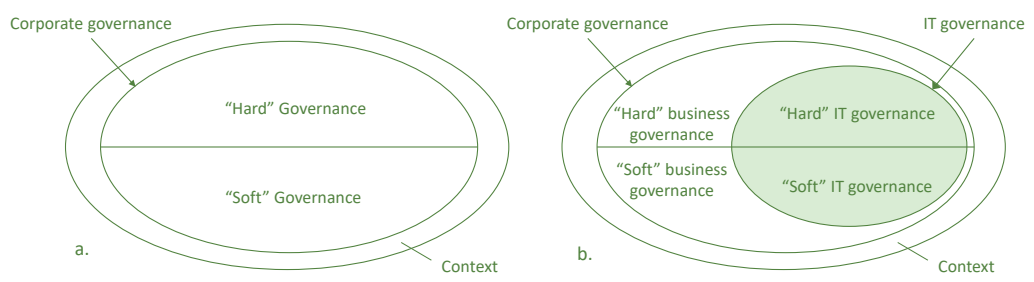

Figure 28 Corporate governance, IT governance and the context. (Figure 26 and Figure 28 are equal)

In Figure 28a, corporate governance is divided into three parts: hard governance, soft governance and the context, just like the MIG model for ITG. The systematic literature review demonstrated that all focus areas are covered in the contemporary corporate governance literature. Given that corporate governance and ITG are related topics, corporate governance should pay attention to the same areas.

In Figure 28b, ITG is highlighted as an integrated aspect of corporate governance. The figure also shows that there might be more focus areas relevant for corporate governance (the part outside ITG).

\subsubsection{Answering the main research question}

MRQ: $\quad H o w$ can the IT governance of an organisation grow in maturity to become more effective?

To answer the main research question, we discuss both parts within the scope of this thesis (see Section 1.4): 
I. How can we improve the ITG maturity of an organisation?

II. Can we confirm the relevance of our findings for corporate governance?

The research questions in the previous sections provide the answer to the main research question. Given that our research does not directly pertain to organisational performance, we discuss the answer using the research questions described in Section 1.4.

\section{MRQ.I: How can we improve the ITG maturity of an organisation?}

The MIG model and the MIG assessment instrument are designed to measure current ITG maturity. The maturity part of the MIG model consists of nine focus areas. During the design process, a maturity model was selected for each of these focus areas. This provided the opportunity to determine the next step in maturity for each area separately. The researchers, together with a substantial group of students, used and evaluated the MIG model and the MIG assessment instrument in three cycles and several dozen case studies.

We combined the use of the instrument with semi-structured interviews. The success of a maturity model is proved "if it brings about a discussion on improvement among the targeted audience" (Lasrado et al., 2017). We received numerous positive comments regarding the usability of the tool in general, such as "It delivers very quickly an indicative impression of the maturity of several ITG processes" and "The assessment and this interview stimulates me. In that way it already has value to me". A long list of proposed improvements to the instrument was also developed.

Combining the instrument with structured interviews resulted in an enhanced and usable instrument to determine the current level of hard and soft ITG of an organisation.

We conclude that this new instrument demonstrates a means of reducing the gap between ITG maturity theory and practice.

\section{MRQ.II: Can we confirm the relevance of our findings for corporate governance?}

In order to confirm the relevance of our findings, we performed a systematic literature review on corporate governance to validate whether the 12 focus areas used in the MIG model exist in the contemporary corporate governance literature. This aligns with the ITG stream that defines ITG as a component of corporate governance. 
The literature review indicated that all 12 focus areas of the MIG model are also covered by the corporate governance literature. This confirms that the focus areas of the MIG model are relevant for corporate governance, too.

This part of the research was published in 2018 and described in detail in Section 8

\subsection{Contribution}

The contribution of the research conducted for this thesis is twofold:

\section{Scientific contribution}

Recent ITG research investigates the effectiveness of structural governance mechanisms and processes. This thesis illustrates that for organisations, the soft side is at least as important. This goes (far) beyond relational governance mechanisms as found in current research. The MIG model and the MIG instrument can serve as a useful model to conduct further research in order to attain a better understanding of these relationships.

In spite of several research efforts reported on in the literature, the causal relationship between ITG and the performance of an organisation remains unclear, as do the ITG mechanisms that affect organisational performance. The current version of the MIG model and the MIG assessment instrument results in theory for analysing the current ITG maturity level of an organization (see Section 2.1). Future versions of the MIG model will contain standard or suggested improvement actions. This could lead to other types of theory e.g. theory for design and action.

The focus areas of the MIG model are relevant for corporate governance as well. Thus, the model and instrument might be useful for research to develop a greater understanding of the way in which the relationship between ITG and corporate governance works.

\section{Practical contribution}

Consultants in practice base their approach on available models, frameworks and experience. The MIG model represents a model created using design science in collaboration with practitioners. ITG is a "top 10" issue for CIOs. As illustrated in Section 3.4.2, CIOs require the availability of a reliable measuring instrument.

The MIG instrument can serve as a practical implementation of the MIG model and represent a useful tool to support employees or consultants in improving ITG in contemporary organisations. 
Section 9

\subsection{Limitations}

During the literature review we selected the best-fitting maturity model within the available time limits and asked our group of experts to rate the suitability. This approach has the limitation that there might be other appropriate maturity models.

Although the model was created in close collaboration with practitioners, the composition of the group of Dutch experts might have affected the resulting maturity model. It has been used in 28 case studies. In-depth case studies were mostly in the government. The complete set of case studies does not reveal substantial differences between sectors, but it would be interesting to conduct in-depth case studies in several non-governmental organisations too.

When defining the statements for the assessment instruments, more options for key elements for the conceptual framework exist. For most maturity levels of the focus areas, there are more than two possible key elements that can be used as a basis for the assessment questions. To validate these choices, the instrument was only used in combination with interviews. However, using different key elements might yield different results.

The case studies show that it is possible to use the tool to map the current ITG of an organisation into the MIG model. The investigated organisations differ in terms of size and industrial sector. However, the validation was limited to a few dozen organisations based in the Netherlands and large multinationals, which vary in size and industrial sector. Indeed, case studies in other countries or regions with different cultures might deliver different results.

\subsection{Future research}

Further research can be categorised into future research to improve the MIG model and future research to improve the MIG instrument.

The MIG model

The MIG model is a focus area maturity model. In this thesis we determined the focus areas and the capabilities. The next step would be to study and define dependencies between the identified capabilities and a positioning of the capabilities in a matrix. In particular, defining dependencies between the focus areas within the hard and soft governance domains may constitute a challenge. Adding standard or suggested improvement actions to the maturity levels of the focus areas might improve the usability of the model. 
The complete model should be validated, tested and further improved in several organisations in different kinds of industries. Maturity models should evolve over time, where through continued use, difficulties or limitations may be revealed (Maier et al., 2012). The transition from development to evaluation is fluid. By using the model in case studies, we can gain feedback from the experiences of companies to refine the maturity model iteratively.

A brief description of the relationship between ITG and corporate governance was described in Section 8. A definition of a focus area maturity model for corporate governance would be interesting for the literature and practice, as ITG and corporate governance are related, yet little is known about how this relationship actually works.

\section{The MIG instrument}

The case studies in this thesis indicate that the instrument continues to require improvements, and this will require several cycles. A new version of the instrument will be available yearly. Each year, we expect that approximately 10 case studies will be conducted by students and/ or the researchers. The data collected will be used to create an improved version of the MIG instrument for the subsequent year.

During the case studies, we collected the data to determine the current state within several organisations. These data can also be used in a longitudinal study.

We conducted case studies with relatively few participants (between three and 10). It would be interesting to conduct case studies with a larger number of participants.

All cases studies described in this thesis were conducted in the Netherlands. It would be interesting to use and validate the model in other countries.

The MIG instrument was created in Excel. An online version of the instrument would be easier to use and represent a good way of creating a case study database that might be usable to create benchmarks.

Another goal is to stimulate adaption by others, including academics and practitioners alike. The publications (see Section "Publication list") and this thesis will be available for scientists. Practitioners will need a more practical guideline, which currently remains under construction. Some adoption has already occurred in the Netherlands (a Governmental Audit Department) and Germany (by Master's students).

Further improvements to the questionnaire might help achieve the ultimate goal: to create an instrument that can be used by members of an organisation without requiring interviews to correct the results and render the instrument available to the public. 
The MIG model is a situational maturity model, but the situational aspects of ITG maturity have not yet been analysed.

\section{Organisational performance}

The causal relationship of how ITG promotes firm performance remains unclear (Vejseli and Rossmann, 2017, Wu et al., 2015), and so a better understanding of this relationship is required. In this thesis we follow the ITG stream that defines ITG as an aspect of corporate governance. Our assumption was that improving corporate governance would result in greater organisational performance. One of the results of this thesis is that the focus areas of the MIG model are relevant for corporate governance as well.

These focus areas can be seen as 12 new perspectives for future research on the relationship between corporate governance, ITG and the performance of an organisation. 


\section{ACKNOW LEDGEMENTS}

I would like to thank all people in the numerous workshops and meetings, from 2012 until the present, for their contributions to the results and all reviewers for their review comments. Without your contributions this result could never have been achieved.

Special thanks go to: Pieter Boere, Wouter Bronsgeest, Robert Deckers, Wilmar Hassoldt, Anton Opperman, Geert Pater, Marije Stam, Martin van der Berg, Marcel van Hal, Lonneke van Sleuven, Marlies van Steenbergen and Sander Zwienink.

Thank you for your time, effort and good advice.

And finally to my wife Elles for her understanding and support and my children Tony, Nicole and Angela. 


\section{R E F E R E N C E S}

Abbott, L. J. \& Parker, S. 2000. Auditor selection and audit committee characteristics. Auditing, Vol. 19, No. 2, pp 46-66.

Abbott, L. J., Parker, S. \& Peters, G. F. 2004. Audit committee characteristics and restatements. Auditing, Vol. 23, No. 1, pp 69-87.

Abraham, S. \& Cox, P. 2007. Analysing the determinants of narrative risk information in UK FTSE 100 annual reports. British Accounting Review, Vol. 39, No. 3, pp 227-248.

Adams, C. A. 2002. Internal organisational factors influencing corporate social and ethical reporting: Beyond current theorising. Accounting, Auditing \& Accountability Journal, Vol. 15, No. 2, pp 223-250.

Aguilera, R. V. 2005. Corporate governance and director accountability: An institutional comparative perspective. British Journal of Management, Vol. 16, No. SPEC. ISS., pp S39-S53.

Alagha, H. 2013. Examining the relationship between IT governance domains, maturity, mechanisms, and performance: An empirical study toward a conceptual framework. Tenth International Conference on Information Technology: New Generations (ITNG). IEEE, pp 767-772.

Albayrak, A. \& Gadatsch, A. 2012. IT Governance model for small and medium sized enterprises. Munich, European, Mediterranean \& Middle East Conference on Informafion Systems (EMOIS). pp 380-390.

Alexander, E. R. 2016. There is no planning-only planning practices: Notes for spatial planning theories. Planning Theory, Vol. 15, No. 1, pp 91-103.

Ali, S. \& Green, P. 2007. IT governance mechanisms in public sector organisations: An Australian context. Journal of Global Information Management, Vol. 15, No. 4, pp 41-63.

Ali, S. \& Green, P. 2012. Effective information technology (IT) governance mechanisms: An IT outsourcing perspective. Information Systems Frontiers, Vol. 14, No. 2, pp 179-193.

Allen, F., Qian, J. \& Qian, M. 2005. Law, finance, and economic growth in China. Journal of Financial Economics, Vol. 77, No. 1, pp 57-116.

Andersen, K. V. \& Henriksen, H. Z. 2006. E-government maturity models: Extension of the Layne and Lee model. Government information quarterly, Vol. 23, No. 2, pp 236-248.

Andres, P. D. \& Vallelado, E. 2008. Corporate governance in banking: The role of the board of directors. Journal of Banking and Finance, Vol. 32, No. 12, pp 2570-2580.

Aoki, M., Jackson, G. \& Miyajima, H. 2007. Corporate governance in Japan: Institutional change and organizational diversity.

Artés, J., Pedraja-Chaparro, F. \& del Mar Salinas-JiméneZ, M. 2017. Research performance and teaching quality in the Spanish higher education system: Evidence from a medium-sized university. Research Policy, Vol. 46, No. 1, pp 19-29.

Ateşer, M. \& Tanriöver, Ö. 2014. Investigation of the Cobit framework's input \output relationships by using graph metrics. Federated Conference on Computer Science and Information Systems (FedCSIS). IEEE, pp 1269-1275.

Bach, J. 1995. Enough about process: What we need are heroes. Software, IEEE, Vol. 12, No. 2, pp 96-98.

Barnard, C. 1938. The functions of the Executive Harvard University Press Cambridge. MA Google Scholar.

Barrett, R. 1998. Liberating the corporate soul: Building a visionary organization, Butterworth-Heinemann Cambridge, Ma. 


\section{References}

Basili, V. R. 1996. The role of experimentation in software engineering: Past, current, and future. Proceedings of the 18th International Conference on Software Engineering. IEEE Computer Society, pp 442-449.

Baskerville, R. L., Kaul, M. \& Storey, V. C. 2015. Genres of Inquiry in design-science research: Justification and evaluation of knowledge production. MIS Quarterly, Vol. 39, No. 3, pp 541-564.

Bass, B. M. \& Steidlmeier, P. 1999. Ethics, character, and authentic transformational leadership behavior. Leadership Quarterly, Vol. 10, No. 2, pp 181-217.

Becker, J., Knackstedt, R. \& Pöppelbuß, D.-W. I. J. 2009. Developing maturity models for IT management. Business E Information Systems Engineering, Vol. 1, No. 3, pp 213-222.

Becker, J., Niehaves, B., Poeppelbuss, J. \& Simons, A. 2010. Maturity models in IS research. ECIS. pp 42.

Bedard, J. C. \& Johnstone, K. M. 2004. Earnings manipulation risk, corporate governance risk, and auditors' planning and pricing decisions. Accounting Review, Vol. 79, No. 2, pp 277-304.

Berg, B. L., Lune, H. \& Lune, H. 2004. Qualitative research methods for the social sciences, Pearson Boston, MA.

Bernard, P. 2012. COBIT® 5 - A management guide, Van Haren.

Bessant, J., Caffyn, S. \& Gallagher, M. 2001. An evolutionary model of continuous improvement behaviour. Technovation, Vol. 21, No. 2, pp 67-77.

Bezemer, P.-J., Maassen, G. F., Van Den Bosch, F. A. J. \& Volberda, H. W. 2007. Investigating the development of the internal and external service tasks of non-executive directors: The case of the Netherlands (1997-2005). Corporate Governance, Vol. 15, No. 6, pp 1119-1129.

Bharadwaj, A. S. 2000. A resource-based perspective on information technology capability and firm performance: an empirical investigation. MIS Quarterly, pp 169-196.

Bhimani, A. \& Soonawalla, K. 2005. From conformance to performance: the corporate responsibilities continuum. Journal of Accounting and Public Policy, Vol. 24, No. 3, pp 165-174.

Bianchi, I. S. \& Sousa, R. D. 2015. IT governance for public universities: Proposal for a framework using Design Science Research. 26th International Business Information Management Association Conference Innovation Management And Sustainable Economic Competitive Madrid, Spain. IBIMA.

Borth, M. A. \& Bradley, R. V. 2009. Unexplored linkages between corporate governance and IT governance: An evaluation and call to research. Information Technology Governance and Service Management: Frameworks and Adaptations. IGI Global.

Bowen, P. L., Cheung, M. Y. D. \& Rohde, F. H. 2007. Enhancing IT governance practices: A model and case study of an organization's efforts. International Journal of Accounting Information Systems, Vol. 8, No. 3, pp 191-221.

Bradley, R. V., Byrd, T. A., Pridmore, J. L., Thrasher, E., Pratt, R. M. E. \& Mbarika, V. W. A. 2012. An empirical examination of antecedents and consequences of IT governance in US hospitals. Journal of Information Technology, Vol. 27, No. 2, pp 156-177.

Bradley, R. V. \& Pratt, R. M. 2011. Exploring the relationships among corporate entrepreneurship, IT governance, and risk management. 44th Hawaii International Conference on System Sciences (HICSS), Koloa, Kauai, HI. IEEE, pp 1-10.

Braithwaite, J. \& Travaglia, J. F. 2008. An overview of clinical governance policies practices and initiatives. Australian Health Review, Vol. 32, No. 1, pp 10-22.

Brammer, S., Jackson, G. \& Matten, D. 2012. Corporate social responsibility and institutional theory: New perspectives on private governance. Socio-Economic Review, Vol. 10, No. 1, pp 3-28. 
Brennan, N. M. \& Solomon, J. 2008. Corporate governance, accountability and mechanisms of accountability: An overview. Accounting, Auditing E Accountability Journal, Vol. 21, No. 7, pp 885-906.

Brinkkemper, S. 1996. Method engineering: Engineering of information systems development methods and tools. Information and software technology, Vol. 38, No. 4, pp 275-280.

Brown, A. E. \& Grant, G. G. 2005. Framing the frameworks: A review of IT governance research. Communications of the Association for Information Systems, Vol. 15, No. 1, pp 38.

Brown, C. V. 1997. Examining the emergence of hybrid IS governance solutions: Evidence from a single case site. Information Systems Research, Vol. 8, No. 1, pp 69-94.

Brown, C. V. \& Magill, S. L. 1998. Reconceptualizing the context-design issue for the information systems function. Organization Science, Vol. 9, No. 2, pp 176-194.

Brown, C. V. \& Renwick, J. S. 1996. Alignment of the IS organization: The special case of corporate acquisitions. ACM SIGMIS Database, Vol. 27, No. 4, pp 25-33.

Brown, S. \& Knight, P. 2012. Assessing learners in higher education, Routledge.

Bucher, T., Klesse, M., Kurpjuweit, S. \& Winter, R. 2007. Situational method engineering. Situational Method Engineering: Fundamentals and Experiences. Springer.

Burns, J. M. 1978. Leadership, Harper \& Row.

Burns, R. B. 1997. Introduction to research methods, Addison Wesley Longman.

Calder, A. 2008. ISO/IEC 38500: International standard for corporate governance of IT (IT governance), It Governance Limited.

Caldwell, C. \& Karri, R. 2005. Organizational governance and ethical systems: A covenantal approach to building trust. Journal of Business Ethics, Vol. 58, No. 1, pp 249-259.

Cameron, K. S. \& Quinn, R. E. 2005. Diagnosing and changing organizational culture: Based on the competing values framework, Hoboken, NJ John Wiley \& Sons.

Cameron, K. S. \& Quinn, R. E. 2011. Diagnosing and changing organizational culture: Based on the competing values framework, John Wiley \& Sons.

Campbell, J., McDonald, C. \& Sethibe, T. 2010. Public and private sector IT governance: Identifying contextual differences. Australasian Journal of Information Systems, Vol. 16, No. 2, pp 5-18.

Cannella, B., Finkelstein, S. \& Hambrick, D. C. 2009. Strategic leadership: Theory and research on executives, top management teams, and boards, Oxford University.

Carpenter, M. A., Pollock, T. G. \& Leary, M. M. 2003. Testing a model of reasoned risk-taking: Governance, the experience of principals and agents, and global strategy in high-technology IPOs firms. Strategic Management Journal, Vol. 24, No. 9, pp 803-820.

Carpenter, M. A. \& Westphal, J. D. 2001. The strategic context of external network ties: Examining the impact of director appointments on board involvement in strategic decision making. Academy of Management Journal, Vol. 44, No. 4, pp 639-660.

Chan, Y. E. 2002. Why haven't we mastered alignment? The importance of the informal organization structure. MIS Quarterly Executive, Vol. 1, No. 2, pp 97-112.

Chan, Y. E., Huff, S. L., Barclay, D. W. \& Copeland, D. G. 1997. Business strategic orientation, information systems strategic orientation, and strategic alignment. Information systems research, Vol. 8, No. 2, pp 125-150.

Chin, P. O., Brown, G. A. \& Hu, Q. 2004. The impact of mergers \& acquisitions on IT governance structures: A case study. Journal of Global Information Management, Vol. 12, No. 4, pp 50-74. 


\section{References}

Choi, F.-H. \& Wong, T. J. 2007. Auditors' governance functions and legal environments: An international investigation. Contemporary Accounting Research, Vol. 24, No. 1, pp 13-46+1+6.

Churchman, C. W. \& Schainblatt, A. 1965. The researcher and the manager: A dialectic of implementation. Management Science, Vol. 11, No. 4, pp B-69-B-87.

Ciborra, C., Braa, K., Cordella, A., Hepsø, V., Dahlbom, B., Failla, A. \& Hanseth, O. 2000. From control to drift: The dynamics of corporate information infastructures, Oxford University Press on Demand.

Ciborra, C. U. 1997. De profundis? Deconstructing the concept of strategic alignment. Scandinavian Journal of Information Systems, Vol. 9, No. 1, pp 67-82.

Ciborra, C. U. 1998. Crisis and foundations: an inquiry into the nature and limits of models and methods in the information systems discipline. The Journal of Strategic Information Systems, Vol. 7, No. 1, pp 5-16.

Cioffi, J. W. \& Höpner, M. 2006. The political paradox of finance capitalism: Interests, preferences, and centerleft party politics in corporate governance reform. Politics and Society, Vol. 34, No. 4, pp 463-502.

Claessens, S. \& Yurtoglu, B. B. 2013. Corporate governance in emerging markets: A survey. Emerging Markets Review, Vol. 15, No., pp 1-33.

Clark, F. C. 2001. IT governance involves top execs. Hospitals and health networks, Vol. 75, No. 10, pp 10-12.

Clark, G. L. \& Wójcik, D. 2003. An economic geography of global finance: Ownership concentration and stockprice volatility in German firms and regions. Annals of the Association of American Geographers, Vol. 93, No. 4, pp 909-924.

Cobb, A. T. 1980. Informal influence in the formal organization: Perceived sources or power among work unit peers. Academy of Management Journal, Vol. 23, No. 1, pp 155-161.

Cobo, A., Vanti, A. A. \& Rocha, R. 2014. A fuzzy multicriteria approach for it governance evaluation. JISTEMJournal of Information Systems and Technology Management, Vol. 11, No. 2, pp 257-276.

Collins, J. 2001. Level 5 leadership: The triumph of humility and fierce resolve. Harvard Business Review, Vol. 79, No. 1, pp 66-76.

Conti, T. 2003. Quality assessments for improvement in corporate governance. ASQ Annual Quality Congress Proceedings.

Cook, D. M. 2010a. Mitigating cyber-threats through public-private partnerships: Low cost governance with high-impact returns. International Cyber Resilience Conference, Perth Western Australia, . pp 22.

Cook, D. M. 2010b. The Use of Governance to Identify Cyber Threats Through Social Media. 2010 INTERNATIONAL CYBER RESILIENCE CONFERENCE. pp 31.

Cook, T. D., Campbell, D. T. \& Day, A. 1979. Quasi-experimentation: Design E analysis issues for field settings, Houghton Mifflin Boston.

Cooke-Davies, T. J. \& Arzymanow, A. 2003. The maturity of project management in different industries: An investigation into variations between project management models. International Journal of Project Management, Vol. 21, No. 6, pp 471-478.

Cornforth, C. 2004. The Governance of cooperatives and mutual associations: A paradox perspective. Annals of Public and Cooperative Economics, Vol. 75, No. 1, pp 11-32.

Crane, A., McWilliams, A., Matten, D., Moon, J. \& Siegel, D. 2009. The Oxford handbook of corporate social responsibility, Oxford Handbooks.

Cristo, D. A. 2005. Soft power: The means to success in world politics. American Economist, Vol. 49, No. 2, pp 99-100.

Crosby, P. B. 1979. Quality is free: The art of making quality certain, McGraw-Hill, New York. 
Curry, E., Guyon, B., Sheridan, C. \& Donnellan, B. 2012. Sustainable IT: Challenges, postures, and outcomes. Computer, Vol. 45, No. 11, pp 79-81.

Cyert, R. M., Kang, S.-H. \& Kumar, P. 2002. Corporate governance, takeovers, and top-management compensation: Theory and evidence. Management Science, Vol. 48, No. 4, pp 453-469.

Dahlberg, T. \& Kivijärvi, H. 2006. An integrated framework for IT governance and the development and validation of an assessment instrument. 39th Annual Hawaii International Conference on System Sciences (HICSS), Kauai, HI.

Daily, C. M., Dalton, D. R. \& Cannella, A. A. 2003. Corporate governance: Decades of dialogue and data. Academy of management review, Vol. 28, No. 3, pp 371-382.

Daily, C. M., McDougall, P. P., Covin, J. G. \& Dalton, D. R. 2002. Governance and strategic leadership in entrepreneurial firms. Journal of Management, Vol. 28, No. 3, pp 387-412.

Dalipi, F. \& Shej, A. 2012. Towards an integrated model of optimizing the efficiency of IT in organizations. ICT Innovations 2011. Springer.

Dalton, D. R., Daily, C. M., Ellstrand, A. E. \& Johnson, J. L. 1998. Meta-analytic reviews of board composition, leadership structure, and financial performance. Strategic Management Journal, Vol. 19, No. 3, pp 269-290.

Dam, H. K. \& Winikoff, M. 2013. Towards a next-generation AOSE methodology. Sci. Comput. Program., Vol. 78, No. 6, pp 684-694.

Darke, P., Shanks, G. \& Broadbent, M. 1998. Successfully completing case study research: Combining rigour, relevance and pragmatism. Information Systems Journal, Vol. 8, No. 4, pp 273-289.

Davies, M. A. 2012. Best practice in corporate governance: Building reputation and sustainable success, Aldershot, UK, Gower Publishing, Ltd.

Davis, G. F. \& Greve, H. R. 1997. Corporate elite networks and governance changes in the 1980s. American Journal of Sociology, Vol. 103, No. 1, pp 1-37.

De Bruin, T., Freeze, R., Kaulkarni, U. \& Rosemann, M. 2005. Understanding the main phases of developing a maturity assessment model. In: Campbell, B., Underwood, J. \& Bunker, D. (eds.) Australasian Conference on Information Systems (ACIS). Sydney, New South Wales, Australia: Australasian Chapter of the Association for Information Systems.

de Bruin, T. \& Rosemann, M. 2007. Using the Delphi technique to identify BPM capability areas. ACIS 2007 Proceedings, pp 42.

de Caluwé, L. \& Vermaak, H. 2004. Thinking about change in different colours. Dynamics of Organizational Change and Learning, pp 197-226.

de Haes, S. \& van Grembergen, W. 2009. An exploratory study into IT governance Implementations and its impact on business/IT alignment. Information Systems Management, Vol. 26, No. 2, pp 123-137.

De Haes, S., Van Grembergen, W. \& Debreceny, R. S. 2013. COBIT 5 and enterprise governance of information technology: Building blocks and research opportunities. Journal of Information Systems, Vol. 27, No. 1, pp 307-324.

de Moraes, A. J. M. 2013. Proposal of result indicators for monitoring of operational initiatives of information technology (IT) by the it governance - through 12 (twelve) operational areas of IT - In order to check how these are related to corporate's strategic planning. 17th World Multi-Conference on Systemics, Cybernetics and Informatics (WMSCI). pp 262-267. 


\section{References}

de Moraes, A. J. M. 2014. Case study: Implementation of IT governance in a major industry located in Brazil's central region (issues and results). 18th World Multi-Conference on Systemics, Cybernetics and Informatics (WMSCI).

de Sousa Pereira, R. F. \& da Silva, M. M. 2010. A maturity model for implementing ITIL v3. 6th World Congress on Services. IEEE, pp 399-406.

de Vreede, G. \& Briggs, R. O. 2005. Collaboration engineering: Designing repeatable processes for highvalue collaborative tasks. 38th Annual Hawaii International Conference on System Sciences (HICSS). IEEE, pp 17.

Delgado, A. P. \& Velthuis, M. P. 2015. Proposal for a continuous improvement IT governance framework at financial institutions [Propuesta de marco de mejora continua de gobierno TI en entidades financieras]. RISTI - Revista Iberica de Sistemas e Tecnologias de Informacao, 15, pp 51-67.

Deming, W. E. 1986. Out of the Crisis, 1986. Cambridge, Mass.: Massachusetts Institute of Technology Center for Advanced Engineering Study. xiii, Vol. 507, No.

DeSanctis, G. \& Poole, M. S. 1994. Capturing the complexity in advanced technology use: Adaptive structuration theory. Organization Science, Vol. 5, No. 2, pp 121-147.

Dietz, J. L. \& Hoogervorst, J. A. 2012. The principles of enterprise engineering. Advances in Enterprise Engineering VI. Springer.

Dodds, R. 2004. Effective information technology governance will improve returns to shareholders. Information Systems Control Journal, Vol. 3, No. 1, pp 17-18.

Donnellan, B., Sheridan, C. \& Curry, E. 2011. A capability maturity framework for sustainable information and communication technology. IT professional, Vol. 13, No. 1, pp 33-40.

Donnelly, R. \& Mulcahy, M. 2008. Board structure, ownership, and voluntary disclosure in Ireland. Corporate Governance, Vol. 16, No. 5, pp 416-429.

Doty, D. H. \& Glick, W. H. 1994. Typologies as a unique form of theory building: Toward improved understanding and modeling. Academy of management review, Vol. 19, No. 2, pp 230-251.

Duncan, W. J. 1978. Organizational behavior, Houghton Mifflin Harcourt (HMH).

Eames, C. \& Eames, R. 1972. Design Q E A, Color film.

Earl, P. E. \& Potts, J. 2011. A Nobel Prize for governance and institutions: Oliver Williamson and Elinor Ostrom. Review of Political Economy, Vol. 23, No. 1, pp 1-24.

Easterly, W. 2008. Institutions: Top down or bottom up? The American Economic Review, Vol. 98, No. 2, pp 95-99.

Eisenhardt, K. M. 1989. Building theories from case study research. Academy of Management Review, Vol. 14, No. 4, pp 532-550.

Elagha, H. 2014. The use of partial least squares path modeling in IT governance discipline. 11th International Conference on Information Technology: New Generations (ITNG). IEEE, pp 624-626.

Eurostat 2008. NACE Rev. 2 Statistical classification of economic activities in the European Community. Eurostat European Commission.

Evans, C. 2016. Re-thinking case-based assessments in business management education. The International Journal of Management Education, Vol. 14, No. 2, pp 161-166.

Fan, Y. 2008. Soft power: Power of attraction or confusion? Place Branding and Public Diplomacy, Vol. 4, No. 2, pp 147-158. 
Farndale, E., Paauwe, J. \& Boselie, P. 2010. An exploratory study of governance in the intra-firm human resources supply chain. Human Resource Management, Vol. 49, No. 5, pp 849-868.

Filatotchev, I. \& Nakajima, C. 2010. Internal and external corporate governance: An interface between an organization and its environment. British Journal of Management, Vol. 21, No. 3, pp 591-606.

Fitzgerald, B., Russo, N. L. \& O’Kane, T. 2003. Software development method tailoring at Motorola. Communications of the ACM, Vol. 46, No. 4, pp 64-70.

Fjermestad, J. \& Hiltz, S. R. 2000. Group support systems: A descriptive evaluation of case and field studies. Journal of Management Information Systems, Vol. 17, No. 3, pp 113-157.

Forbes, D. P. \& Milliken, F. J. 1999. Cognition and corporate governance: Understanding boards of directors as strategic decision-making groups. Academy of Management Review, Vol. 24, No. 3, pp 489-505.

Fraser, P., Moultrie, J. \& Gregory, M. 2002. The use of maturity models/grids as a tool in assessing product development capability. Engineering Management Conference (IEMC'02). IEEE, pp 244-249.

French, J., Raven, B. \& Cartwright, D. 1959. The bases of social power. Classics of organization theory, Vol. 7, No., pp 311-320.

Friedmann, J. \& Hudson, B. 1974. Knowledge and action: A guide to planning theory. Journal of the American Institute of Planners, Vol. 40, No. 1, pp 2-16.

Fulgence, S. E. 2014. Corporate governance in Tanzania. In: Idowu, S. O. \& Çaliyurt, K. T. (eds.) Corporate Governance: An International Perspective. Berlin: Springer.

Galbraith, J. R. 1974. Organization design: An information processing view. Interfaces, Vol. 4, No. 3, pp 28-36.

Galle, P. \& Kroes, P. 2014. Science and design: Identical twins? Design Studies, Vol. 35, No. 3, pp 201-231.

Gedajlovic, E., Carney, M., Chrisman, J. J. \& Kellermanns, F. W. 2012. The adolescence of family firm research: Taking stock and planning for the Future. Journal of Management, Vol. 38, No. 4, pp 1010-1037.

Giannetti, M. \& Koskinen, Y. 2010. Investor protection, equity returns, and financial globalization. Journal of Financial and Quantitative Analysis, Vol. 45, No. 1, pp 135-168.

Gibbert, M., Ruigrok, W. \& Wicki, B. 2008. What passes as a rigorous case study? Strategic Management Journal, Vol. 29, No. 13, pp 1465-1474.

Globerman, S., Peng, M. W. \& Shapiro, D. M. 2011. Corporate governance and Asian companies. Asia Pacific Journal of Management, Vol. 28, No. 1, pp 1-14.

Gomez-Mejia, L. R., Cruz, C., Berrone, P. \& de Castro, J. 2011. The bind that ties: Socioemotional wealth preservation in family firms. Academy of Management Annals, Vol. 5, No. 1, pp 653-707.

Gorton, G. \& Schmid, F. A. 2004. Capital, labor, and the firm: A study of German codetermination. Journal of the European Economic Association, Vol. 2, No. 5, pp 863-905.

Graebner, M. E. \& Eisenhardt, K. M. 2004. The seller's side of the story: Acquisition as courtship and governance as syndicate in entrepreneurial firms. Administrative Science Quarterly, Vol. 49, No. 3, pp 366-403.

Grant, K. P. \& Pennypacker, J. S. 2006. Project management maturity: An assessment of project management capabilities among and between selected industries. Engineering Management, IEEE Transactions on, Vol. 53, No. 1, pp 59-68.

Gregor, S. 2002. Design theory in information systems. Australian Journal of Information Systems, Vol. 10, No. 1 Special issue, pp 14-22.

Gregor, S. 2006. The nature of theory in information systems. MIS quarterly, Vol. 3, No. 30, pp 611-642. 


\section{References}

Gregor, S. \& Hevner, A. R. 2013. Positioning and presenting design science research for maximum impact. MIS quarterly, Vol. 37, No. 2, pp 337-355.

Gregor, S. \& Jones, D. 2007. The anatomy of a design theory. Journal of the Association for Information systems, Vol. 8, No. 5, pp 312.

Guest, P. M. 2008. The determinants of board size and composition: Evidence from the UK. Journal of Corporate Finance, Vol. 14, No. 1, pp 51-72.

Gulati, R. \& Puranam, P. 2009. Renewal through reorganization: The value of inconsistencies between formal and informal organization. Organization Science, Vol. 20, No. 2, pp 422-440.

Györy, A. A. B., Cleven, A., Uebernickel, F. \& Brenner, W. 2012. Exploring the shadows: IT governance approaches to user-driven innovation.

Halter, M. V., de Arruda, M. C. C. \& Halter, R. B. 2009. Transparency to reduce corruption? Journal of Business Ethics, Vol. 84, No., pp 373-385.

Harmsen, A. F., Ernst, M. \& Twente, U. 1997. Situational method engineering, University of Twente, Enschede, Netherlands.

Heath, C. \& Sitkin, S. B. 2001. Big-B versus Big-O: What is organizational about organizational behavior? Journal of Organizational Behavior: The International Journal of Industrial, Occupational and Organizational Psychology and Behavior, Vol. 22, No. 1, pp 43-58.

Heath, J. \& Norman, W. 2004. Stakeholder theory, corporate governance and public management: What can the history of state-run enterprises teach us in the post-Enron era? Journal of Business Ethics, Vol. 53 , No. 3, pp 247-265.

Helfat, C. E. \& Peteraf, M. A. 2003. The dynamic resource-based view: Capability lifecycles. Strategic Management Journal, Vol. 24, No. 10, pp 997-1010.

Helgesson, Y. Y. L., Höst, M. \& Weyns, K. 2012. A review of methods for evaluation of maturity models for process improvement. Journal of Software: Evolution and Process, Vol. 24, No. 4, pp 436-454.

Henderson-Sellers, B. \& Ralyté, J. 2010. Situational method engineering: State-of-the-art review. Journal of Universal Computer Science, Vol. 16, No. 3, pp 424-478.

Hendry, J. 2004. Between enterprise and ethics: Business and management in a bimoral society, Oxford University Press.

Heracleous, L. 2003. Strategy and organization: Realizing strategic management, Cambrigde University Press.

Herz, T. P., Hamel, F., Uebernickel, F. \& Brenner, W. 2011. IT governance mechanisms in multisourcing - A business group perspective. 45th Hawaii International Conference on System Sciences (HICSS), Maui, HI. pp 5033-5042.

Hevner, A. \& Chatterjee, S. 2010. Design science research in information systems. Design research in information systems. Springer.

Hevner, A. R. 2007. A three cycle view of design science research. Scandinavian Journal of Information Systems, Vol. 19, No. 2, pp 4.

Hevner, A. R., March, S. T., Park, J. \& Ram, S. 2004. Design science in information systems research. MIS quarterly, Vol. 28, No. 1, pp 75-105.

Hirsch, P. M. 1982. Network data versus personal accounts: The normative culture of interlocking directorates. Annual Meeting of the American Sociology Association San Francisco, CA.

Ho, B. 2012. Apologies as signals: With evidence from a trust game. Management Science, Vol. 58, No. 1, pp $141-158$. 
Huang, R., Zmud, R. W. \& Price, R. L. 2010. Influencing the effectiveness of IT governance practices through steering committees and communication policies. European Journal of Information Systems, Vol. 19, No. 3, pp 288-302.

Hudson, B. M., Galloway, T. D. \& Kaufman, J. L. 1979. Comparison of current planning theories: Counterparts and contradictions. Journal of the American Planning Association, Vol. 45, No. 4, pp 387-398.

Huse, M. 2005a. Accountability and creating accountability: A framework for exploring behavioural perspectives of corporate governance. British Journal of Management, Vol. 16, No. s1.

Huse, M. 2005b. Corporate governance: Understanding important contingencies. Corporate Ownership $\mathcal{E}$ Control, Vol. 2, No. 4, pp 41-50.

Huse, M. 2007. Boards, governance and value creation: The human side of corporate governance, Cambridge University Press.

Huse, M. \& Solberg, A. G. 2006. Gender-related boardroom dynamics: How Scandinavian women make and can make contributions on corporate boards. Women in Management Review, Vol. 21, No. 2, pp 113-130.

Ibrahim, L. \& Nurpulaela, L. 2016. Evaluation of IT governance to support IT operation excellent based on COBIT 4.1 at the PT Timah Tbk. Information Technology, Computer, and Electrical Engineering (ICITACEE), 2016 3rd International Conference on. IEEE, pp 336-339.

IFAC 2004. Enterprise governance: Getting the balance right. New York: IFAC.

Iivari, J. \& Venable, J. 2009. Action research and design science research-seemingly similar but decisively dissimilar. 17th European Conference on Information Systems. pp 1-13.

Imenda, S. 2014. Is there a conceptual difference between theoretical and conceptual frameworks? Journal of Social Sciences, Vol. 38, No. 2, pp 185-195.

Ingley, C. B. \& Van Der Walt, N. T. 2001. The strategic board: The changing role of directors in developing and maintaining corporate capability. Corporate Governance, Vol. 9, No. 3, pp 174-185.

ISACA 2008. Enterprise value: governance of IT investments. The val IT framework 2.0. Meadows, IL, US.: ISACA.

ISACA 2009. The risk IT framework: Principles. Meadows, IL, US.: ISACA.

ISACA 2012. COBIT 5: A Business Framework for the Governance and Management of Enterprise IT, Meadows, IL, US., ISACA.

ISACA 2018. COBIT 2019 Framework: Governance and Management Objectives. Meadows, IL, US.: ISACA

Ishaq, A., Mukhtar, M., Wahyudi, M., Indriani, K., Elham, A., Kayed, A., Babikir, S. A., Minkin, D. Y., Sineshchuk, Y. I. \& Terekhin, S. N. 2017. Information technology governance using cobit 4.0 domain delivery support and monitoring evaluation. Journal of Theoretical and Applied Information Technology, Vol. 95, No. 20 .

ISO/IEC 15504 2004. Information technology - process assessment - Part 1: concepts and vocabulary.

ISO/IEC 38500 2008. International Standard for Corporate Governance of IT. International Organization for Standardization (ISO) and the International Electrotechnical Commission (IEC).

Itakura, H. 2007. IT governance: Organizational capabilities' view. Portland International Conference on Management of Engineering\&Technology (PICMET'07), Portland, OR. pp 911-915.

ITGI 2003a. Board briefing on IT governance. IT Governance Institute, Meadows, IL, US.

ITGI 2003b. Board briefing on IT governance, Second Edition. IT Governance Institute, Meadows, IL, US. 
ITGI 2007. COBIT® 4.1: Framework, Control Objectives, Management Guidelines, Maturity Models, IT Governance Institute, Meadows, IL, US.

ITGI 2008. Governance Global Status Report. IT Governance Institute, Meadows, IL, US.

ITGI 2011. Global Status Report on the Governance of Enterprise IT (GEIT). IT Governance Institute, Meadows, IL, US.

Jabareen, Y. 2009. Building a conceptual framework: Philosophy, definitions, and procedure. International Journal of Qualitative Methods, Vol. 8, No. 4, pp 49-62.

Jackson, G. 2005. Stakeholders under pressure: Corporate governance and labour management in Germany and Japan. Corporate Governance, Vol. 13, No. 3, pp 419-428.

Jackson, G. \& Apostolakou, A. 2010. Corporate social responsibility in Western Europe: An institutional mirror or substitute? Journal of Business Ethics, Vol. 94, No. 3, pp 371-394.

Janahi, L., Griffiths, M. \& Al-Ammal, H. 2015. A conceptual model for IT governance in public sectors. Fourth International Conference on Future Generation Communication Technology (FGCT). IEEE, pp 1-9.

Jara-Bertin, M., López-Iturriaga, F. J. \& López-De-Foronda, O. 2008. The contest to the control in European family firms: How other shareholders affect firm value. Corporate Governance, Vol. 16, No. 3, pp $146-159$.

Jennings, D. 1996. Strategic management and the case method. Journal of Management Development, Vol. 15 , No. 9, pp 4-12.

Jo, H. \& Harjoto, M. A. 2011. Corporate governance and firm value: The impact of corporate social responsibility. Journal of Business Ethics, Vol. 103, No. 3, pp 351-383.

Joshi, A., Bollen, L., Hassink, H., De Haes, S. \& Van Grembergen, W. 2018. Explaining IT governance disclosure through the constructs of IT governance maturity and IT strategic role. Information E Management, Vol. 55, No. 3, pp 368-380.

Juran, J. M. 1988. Juran on planning for quality, Free Press New York.

Kaiser, R. B., Hogan, R. \& Craig, S. B. 2008. Leadership and the fate of organizations. American Psychologist, Vol. 63, No. 2, pp 96.

Keasey, K. \& Wright, M. 1993. Issues in corporate accountability and governance: An editorial. Accounting and Business Research, Vol. 23, No. sup1, pp 291-303.

Kerlinger, F. N. 1973. Foundations of Behavioural Research (2nd ed.), Holt, Rinehart and Winston New York.

Kim, H., Hoskisson, R. E. \& Wan, W. P. 2004. Power dependence, diversification strategy, and performance in keiretsu member firms. Strategic Management Journal, Vol. 25, No. 7, pp 613-636.

King, B. G., Felin, T. \& Whetten, D. A. 2010. Perspective-Finding the organization in organizational theory: A meta-theory of the organization as a social actor. Organization Science, Vol. 21, No. 1, pp 290-305.

King, J. L. 1983. Centralized versus decentralized computing: organizational considerations and management options. ACM Computing Surveys (CSUR), Vol. 15, No. 4, pp 319-349.

Kitchenham, B. 2004. Procedures for performing systematic reviews. Keele University Joint Technical Report Keele University, UK.

Kitchenham, B., Brereton, O. P., Budgen, D., Turner, M., Bailey, J. \& Linkman, S. 2009. Systematic literature reviews in software engineering-a systematic literature review. Information and Software Technology, Vol. 51, No. 1, pp 7-15.

Klein, A. 2002. Audit committee, board of director characteristics, and earnings management. Journal of Accounting and Economics, Vol. 33, No. 3, pp 375-400. 
Kleindorfer, G. B., O’Neill, L. \& Ganeshan, R. 1998. Validation in simulation: Various positions in the philosophy of science. Management Science, Vol. 44, No. 8, pp 1087-1099.

Kochan, T. A. 2003. Restoring trust in American corporations: Addressing the root cause. Journal of Management and Governance, Vol. 7, No. 3, pp 223-231.

Kosasi, S. 2015. The maturity level of information technology governance of online cosmetics business. International Conference on New Media (CONMEDIA). pp 145.

Krauss, R. M. \& Fussell, S. R. 1990. Mutual knowledge and communicative effectiveness. Intellectual teamwork: Social and technological foundations of cooperative work, pp 111-146.

Kroll, M., Walters, B. A. \& Wright, P. 2008. Board vigilance, director experience, and corporate outcomes. Strategic Management Journal, Vol. 29, No. 4, pp 363-382.

Krug, B. \& Hendrischke, H. 2008. Framing China: Transformation and institutional change through coevolution. Management and Organization Review, Vol. 4, No. 1, pp 81-108.

Kudrle, R. T. 2010. Tax policy in the OECD: Soft governance gets harder. In: Martens, K. \& Jakobi, A. P. (eds.) Mechanisms of OECD Governance: International Incentives for National Policy-Making? Oxfort, UK: Oxfort University Press.

Lainhart, I. \& John, W. 2000. Why IT governance is a top management issue. Journal of Corporate Accounting E Finance, Vol. 11, No. 5, pp 33-40.

Landier, A., Sauvagnat, J., Sraer, D. \& Thesmar, D. 2012. Bottom-up corporate governance. Review of Finance, Vol. 17, No. 1, pp 161-201.

Lasierra Esteban, J. M. 2016. The idea of entrepreneurship in the Austrian economics and the labour market institutions in the global economy [La idea Austriaca de "espíritu empresarial" y las instituciones laborales en la economía global]. Revista de Economia Institucional, Vol. 18, No. 35, pp 79-100.

Lasrado, L., Vatrapu, R., Karsgaard, H. B. \& Kjaer, J. F. 2017. Towards sustainable design for maturity measurement marketplace. International SERIES on Information Systems and Management in Creative eMedia (CreMedia), 2016/2, pp 25-33.

Lasrado, L. A., Vatrapu, R. \& Andersen, K. N. 2015. Maturity models development in IS research. Selected Papers of the IRIS, Oulu, Finland.

Lazic, M., Schillinger, C., Groth, M. \& Heinzl, A. 2011. The impact of IT governance on business performance. Detroit, MI. pp 1681-1690.

Learmount, S. 2004. Corporate governance: What can be learned from Japan?, Oxfort University Press.

Lee, J. \& Lee, C. 2009. IT governance-based IT strategy and management: Literature review and future research directions. Information Technology Governance and Service Management: Frameworks and Adaptations. IGI Global.

Lee, L. T. S. 2012. The pivotal roles of corporate environment responsibility. Industrial Management and Data Systems, Vol. 112, No. 3, pp 466-483.

Lefkowitz, J. 2006. The constancy of ethics amidst the changing world of work. Human Resource Management Review, Vol. 16, No. 2, pp 245-268.

Leifer, R. 1988. Matching computer-based information systems with organizational structures. Mis Quarterly, Vol. 12, No. 1, pp 63-73.

Li, C. 2017. Dynamic analysis of corporate governance impact on investment efficiency: A multiple regression based on high-tech enterprises. Boletin Tecnico/Technical Bulletin, Vol. 55, No. 11, pp 326-334. 


\section{References}

Liang, T. P., Chiu, Y. C., Wu, S. P. J. \& Straub, D. 2011. The impact of IT governance on organizational performance. AMCIS 2011 Proceedings, Detroit, MI. AIS Electronic Library, pp 2388-2396.

Linck, J. S., Netter, J. M. \& Yang, T. 2008. The determinants of board structure. Journal of Financial Economics, Vol. 87, No. 2, pp 308-328.

Lindblom, C. E. 1959. The science of muddling through. Public administration review, Vol. 19, No. 1, pp 79-88.

Linstone, H. A. \& Turoff, M. 1975. The Delphi method: Techniques and applications, Reading, MA, AddisonWesley Publishing Company.

Luftman, J. \& Brier, T. 1999. Achieving and sustaining business-IT alignment. California management review, Vol. 42, No. 1, pp 109-122.

Luhmann, N. 1970. Soziologische aufklärung. Soziologische Aufklärung 1. Springer.

Maes, R. \& Huizing, A. 2005. What do Claudio Ciborra and Sandro Botticelli have in common? On the renaissance of la Primavera. European Journal of Information Systems, Vol. 14, No. 5, pp 480-483.

Magdaleno, A. M., de Araujo, R. M. \& Werner, C. M. L. 2011. A roadmap to the Collaboration Maturity Model (CollabMM) evolution. 15th International Conference on Computer Supported Cooperative Work in Design (CSCWD). IEEE, pp 105-112.

Mahoney, J. 2012. Briefing for IT leaders: Tomorrow's trends and today decisions. Gartner Management Review, Vol. 46, No. 2, pp 26-34.

Maidin, S. S. \& Arshad, N. H. 2010. Information technology governance practices in Malaysian public sector. International Conference on Financial Theory and Engineering (ICFTE), Dubai, United Arab Emirates. pp 281-285.

Maier, A. M., Moultrie, J. \& Clarkson, P. 2012. Assessing organizational capabilities: Reviewing and guiding the development of maturity grids. IEEE Transactions on Engineering Management, Vol. 59, No. 1, pp 138-159.

Mak, Y. T. \& Li, Y. 2001. Determinants of corporate ownership and board structure: Evidence from Singapore. Journal of Corporate Finance, Vol. 7, No. 3, pp 235-256.

Maug, E. 1998. Large shareholders as monitors: Is there a trade-off between liquidity and control? Journal of Finance, Vol. 53, No. 1, pp 65-98.

McNulty, T. \& Pettigrew, A. 1999. Strategists on the board. Organization Studies, Vol. 20, No. 1, pp 47-74.

Merriam-Webster. 2014. Merriam-Webster Online Dictionary [Online]. Available: http://www.merriamwebster.com/. Accessed 23 May 2014. [Accessed].

Mettler, T. 2009. A design science research perspective on maturity models in Information Systems. St. Gallen, Switzerland: Universität St. Gallen

Mettler, T. \& Rohner, P. 2009. Situational maturity models as instrumental artifacts for organizational design. Proceedings of the 4th International Conference on Design Science Research in Information Systems and Technology. ACM, pp 22.

Mettler, T., Rohner, P. \& Winter, R. 2010. Towards a classification of maturity models in information systems. Management of the interconnected world. Springer.

Mintzberg, H. 1979. The structuring of organizations: A synthesis of the research. University of Illinois at Urbana-Champaign's Academy for Entrepreneurial Leadership Historical Research Reference in Entrepreneurship. 
Mohamed, N., Kaur, J. \& Singh, G. 2012. A conceptual framework for information technology governance effectiveness in private organizations. Information Management and Computer Security, Vol. 20, No. 2, pp 88-106.

Moos, L. 2009. Hard and soft governance: The journey from transnational agencies to school leadership. European Educational Research Journal, Vol. 8, No. 3, pp 397-406.

Musson, D. 2009. IT Governance: A critical review of the literature. In: Cater-Steel, A. (ed.) Information technology governance and service management: Frameworks and adaptations. New York: Hershey.

Mustakallio, M., Autio, E. \& Zahra, S. A. 2002. Relational and contractual governance in family firms: Effects on strategic decision making. Family Business Review, Vol. 15, No. 3, pp 205-222.

Nabiollahi, A. \& Sahibuddin, S. B. 2008. Considering service strategy in ITIL v3 as a framework for IT governance. International Symposium on Information Technology (ITSim), Kuala Lumpur, Malaysia.

Nelson, K. M. \& Cooprider, J. G. 1996. The contribution of shared knowledge to IS group performance. MIS Quarterly, Vol. 20, No. 4.

Nelson, T. 2003. The persistence of founder influence: Management, ownership, and performance effects at initial public offering. Strategic Management Journal, Vol. 24, No. 8, pp 707-724.

Nerantzidis, M., Filos, J. \& Lazarides, T. G. 2012. The puzzle of corporate governance definition (s): A content analysis. Corporate Board: Role, Duties and Composition, Vol. 8, No. 2, pp 15-23.

Nfuka, E. N. \& Rusu, L. 2010. Critical success factors for effective it governance in the public sector organisations in a developing country: The case for Tanzania. 18th European Conference on Information Systems (ECIS), Pretoria, South Africa.

Ngoma, M. \& Erasmus, W. 2017. Maturity of IT governance in the South African public sector. International Association for the Management of Information Technology, Orlando, FL.

Ngwenyama, O. \& Nielsen, P. A. 2003. Competing values in software process improvement: an assumption analysis of CMM from an organizational culture perspective. Transactions on Engineering Management, Vol. 50, No. 1, pp 100-112.

Nicholson, G. J., Alexander, M. \& Kiel, G. C. 2004. Defining the social capital of the board of directors: An exploratory study. Journal of Management and Organization, Vol. 10, No. 1, pp 54-72.

Nickell, S., Nicolitsas, D. \& Dryden, N. 1997. What makes firms perform well? European Economic Review, Vol. 41, No. 3-mei, pp 783-796.

Niemann, K. D. 2006. From enterprise architecture to IT governance, Springer.

Ningsih, K. R., Sembiring, J., Arman, A. A. \& Wuryandari, A. I. 2013. Developing IT investment management framework of government institution. International Conference on Advanced Computer Science and Information Systems (ICACSIS). IEEE, pp 237-242.

Novotny, A., Bernroider, E. \& Koch, S. 2012. Dimensions and operationalisations of IT governance: a literature review and meta-case study. Proceedings from the 2012 International Conference on Information Resource Management, Vienna, Austria. WU Vienna University of Economics and Business, pp 1-13.

Nwafor, A. O. 2014a. Combating environmental degradation through diplomacy and corporate governance (part 1). Mediterranean Journal of Social Sciences, Vol. 20, No., pp 202-210.

Nwafor, A. O. 2014b. Combating environmental degradation through diplomacy and corporate governance (Part 2). Mediterranean Journal of Social Sciences, Vol. 20, No., pp 211-217.

Nye, J. S. 1990. Soft power. Foreign Policy, Vol. 80, No. 1, pp 153-171.

Nye, J. S. 2004. Soft power: The means to success in world politics, New York, USA, Public Affairs. 


\section{References}

Okoli, C. \& Pawlowski, S. D. 2004. The Delphi method as a research tool: An example, design considerations and applications. Information \& Management, Vol. 42, No. 1, pp 15-29.

Oliver, D. \& Lainhart, J. 2012. COBIT 5: Adding value through effective GEIT. EDPACS, Vol. 46, No. 3, pp 1-12.

Olson, M. H. \& Chervany, N. L. 1980. The relationship between organizational characteristics and the structure of the information services function. MIS Quarterly, Vol. 4, No. 2, pp 57-68.

Orhangazi, Ö. 2008. Financialisation and capital accumulation in the non-financial corporate sector: 19732003. Cambridge Journal of Economics, Vol. 32, No. 6, pp 863-886.

Orlikowski, W. J. 1992. The duality of technology: Rethinking the concept of technology in organizations. Organization science, Vol. 3, No. 3, pp 398-427.

Pasquini, A. \& Galiè, E. 2013. COBIT 5 and the process capability model. Improvements provided for IT governance process. FIKUSZ Symposium for Young Researchers pp 67-76.

Paulk, M. C. 1999. Analyzing the conceptual relationship between ISO/IEC 15504 (software process assessment) and the capability maturity model for software. International Conference on Software Quality.

Paulk, M. C., Curtis, B., Chrissis, M. B., Averill, E. L., Bamberger, J., Kasse, T. C., Konrad, M. D., Perdue, J. R., Weber, C. V. \& Withey, J. V. 1991. Capability maturity model for software, version 1.0. Pittsburgh USA: Carnegie Mellon University.

Paulk, M. C., Curtis, B., Chrissis, M. B. \& Weber, C. V. 1993. The capability maturity model for software, version 1.1. Software Engineering Project Management, Vol. 10, No. 1, pp 1-26.

Peasnell, K. V., Pope, P. F. \& Young, S. 2005. Board monitoring and earnings management: Do outside directors influence abnormal accruals? Journal of Business Finance and Accounting, Vol. 32, No. 7-8, pp 13111346.

Pederiva, A. 2003. The COBIT® maturity model in a vendor evaluation case. Information Systems Control Journal, Vol. 3, No., pp 26-29.

Peffers, K., Tuunanen, T., Rothenberger, M. A. \& Chatterjee, S. 2007. A design science research methodology for information systems research. Journal of management information systems, Vol. 24, No. 3, pp 45-77.

Peltokorpi, V. \& Tsuyuki, E. 2007. Organizational governance in internal hybrids: A case study of Maekawa Manufacturing Ltd. Corporate Governance, Vol. 2, No., pp 123-135.

Peterson, R. R. 2001. Configurations and coordination for global information technology governance: Complex designs in a transnational European context. Proceedings of the 34th Annual Hawaii International Conference on System Sciences., Maui, HI, USA. IEEE, pp 217.

Peterson, R. R. 2004. Crafting information technology governance. Information Systems Management, Vol. 21, No. 4, pp 7-22.

Peterson, R. R., O'Callaghan, R. \& Ribbers, P. 2000. Information technology governance by design: investigating hybrid configurations and integration mechanisms. 21st International Conference on Information Systems. Association for Information Systems, pp 435-452.

Petticrew, M. \& Roberts, H. 2006. Systematic reviews in the social sciences: A practical guide, Oxford, UK, Blackwell Publishing Ltd.

Pöppelbuß, J., Niehaves, B., Simons, A. \& Becker, J. 2011. Maturity models in information systems research: Literature search and analysis. Communications of the Association for Information Systems, Vol. 29, No. 1, pp 1-15. 
Pöppelbuß, J. \& Röglinger, M. 2011. What makes a useful maturity model? A framework of general design principles for maturity models and its demonstration in business process management. ECIS, Helsinki, Finland. pp 28.

Porter, M. E. 1992. Capital disadvantage: America's failing capital investment system. Harvard Business Review, Vol. 70, No. 5, pp 65-82.

Prasad, A., Green, P. \& Heales, J. 2012. On IT governance structures and their effectiveness in collaborative organizational structures. International Journal of Accounting Information Systems, Vol. 13, No. 3, pp 199-220.

Pries-Heje, J. \& Baskerville, R. 2008. The design theory nexus. MIS Quarterly, pp 731-755.

Prinz, M. 2015. Investigation of the impact of national culture on IT governance: An explorative study contrasting German and Japanese national culture. 21th Americas Conference on Information Systems (AMCIS). Puerto Rico: AIS.

Proença, D. \& Borbinha, J. 2016. Maturity models for information systems-A state of the art. Procedia Computer Science, Vol. 100, No. 1, pp 1042-1049.

Putri, M. A., Lestari, V. A. \& Aknuranda, I. 2017. Audit of information technology governance using COBIT 4.1: Case study in PT. XY. International Conference on Industrial Internet of Things (ICIIOT), Bandung, Indonesia. pp 1-7.

Putri, N. K. S. \& Muljoredjo, H. 2014. The use of green information technology governance model to determine capability maturity level in Jakarta private higher education institutions. Journal of Theoretical $\mathcal{E}$ Applied Information Technology, Vol. 61, No.1.

Quinn, R. E. \& Rohrbaugh, J. 1983. A spatial model of effectiveness criteria: Towards a competing values approach to organizational analysis. Management Science, Vol. 29, No. 3, pp 363-377.

Race, P., Brown, S. \& Smith, B. 2004. 500 tips on assessment, New York, USA, RoutledgeFalmer.

Rai, A., Patnayakuni, R. \& Seth, N. 2006. Firm performance impacts of digitally enabled supply chain integration capabilities. MIS quarterly, Vol. 30, No. 2, pp 225-246.

Rechner, P. L. \& Dalton, D. R. 1991. CEO duality and organizational performance: A longitudinal analysis. Strategic Management Journal, Vol. 12, No. 2, pp 155-160.

Rediker, K. J. \& Seth, A. 1995. Boards of directors and substitution effects of alternative governance mechanisms. Strategic Management Journal, Vol. 16, No. 2, pp 85-99.

Reich, B. H. \& Benbasat, I. 1996. Measuring the linkage between business and information technology objectives. MIS Quarterly, Vol. 20, No.1, pp 55-81.

Rittel, H. W. \& Webber, M. M. 1973. 2.3 planning problems are wicked. Polity, Vol. 4, No., pp 155-169.

Rogers, G. P. 2009. The role of maturity models in IT governance: A comparison of the major models and their potential benefits to the enterprise. Information Technology Governance and Service Management: Frameworks and Adaptations. IGI Global.

Röglinger, M., Pöppelbuß, J. \& Becker, J. 2012. Maturity models in business process management. Business Process Management Journal, Vol. 18, No. 2, pp 328-346.

Rosemann, M. \& Vessey, I. 2008. Toward improving the relevance of information systems research to practice: The role of applicability checks. MIS Quarterly, pp 1-22.

Sacconi, L. 2007. A social contract account for CSR as an extended model of corporate governance (II): Compliance, reputation and reciprocity. Journal of Business Ethics, Vol. 75, No. 1, pp 77-96. 


\section{References}

Saetang, S. \& Haider, A. 2012. Effective IT governance implementation with vital determinants. Twelfth International Conference on Electronic Business: latest global development in electronic business, Xi'an, China. pp 90-103.

Safari, M. R. \& Jiang, Q. 2018. The theory and practice of IT governance maturity and strategies alignment: Evidence from banking industry. Journal of Global Information Management (JGIM), Vol. 26, No. 2, pp 127-146.

Salah, D., Paige, R. \& Cairns, P. 2014. An evaluation template for expert review of maturity models. International Conference on Product-Focused Software Process Improvement. Springer, pp 318-321.

Salvioni, D. M., Gennari, F., Bosetti, L. \& Almici, A. 2014. The audit committee in the EU emerging countries. Corporate Governance and Corporate Social Responsibility: Emerging Markets Focus.

Sambamurthy, V. \& Zmud, R. W. 1999. Arrangements for information technology governance: A theory of multiple contingencies. MIS Quarterly: Management Information Systems, Vol. 23, No. 2, pp 261-290.

Schnabl, E. \& Zenker, A. 2013. Statistical classification of knowledge-intensive business services (KIBS) with NACE Rev. 2, Fraunhofer ISI Karlsruhe.

Schwarz, A. \& Hirschheim, R. 2003. An extended platform logic perspective of IT governance: Managing perceptions and activities of IT. Journal of Strategic Information Systems, Vol. 12, No. 2, pp 129-166.

Seamer, M. 2014. Does effective corporate facilitate continuous market disclosure? Australian Accounting Review, Vol. 24, No. 2, pp 111-126.

SEI 2010. CMMI for development, Version 1.3. Carnegie Mellon University, Pittsburgh.

Sethibe, T., Campbell, J. \& McDonald, C. 2007. IT governance in public and private sector organizations: Examining the differences and defining future research directions. ACIS 2007 Proceedings, Toowoomba, QLD. pp 833-843.

Seyal, A. H., Poon, S. H. \& Tajuddin, S. 2016. A preliminary evaluation of ICT centers performance using COBIT framework: Evidence from institutions of higher learning in Brunei Darussalam. International Conference on Computational Intelligence in Information System. Springer, pp 235-244.

Shaw, R.-S., Cheng, C.-P. \& Shih, S.-P. 2013a. Correlation and impact between IT management and IT governance. Information Technology Journal, Vol. 12, No. 18, pp 4569-4575.

Shaw, R.-S., Cheng, C.-P., Shih, S.-P. \& Chang, S.-C. 2013b. An empirical investigation of ITG performance in Taiwanese enterprises. Information Technology Journal, Vol. 12, No. 18, pp 4601-4609.

Shewhart, W. A. 1931. Economic control of quality of manufactured product, New York, USA, ASQ Quality Press.

Shleifer, A. \& Vishny, R. W. 1997. A survey of corporate governance. The journal of finance, Vol. 52, No. 2, pp 737-783.

Simon, H. A. 1988. The science of design: Creating the artificial. Design Issues, Vol. 4, No. 1/2, pp 67-82.

Simon, H. A. 1996. The sciences of the artificial, MIT press.

Simonsson, M., Johnson, P. \& Ekstedt, M. 2010. The effect of IT governance maturity on IT governance performance. Information Systems Management, Vol. 27, No. 1, pp 10-24.

Smits, D., Bast, C., van Beele, J., Bloem, J., Hassoldt, W., Hofman, C., van Leeuwen, J., Peeters, R., Pol, C., van Puffelen, J., Schekkerman, J., van Steen, L., Verhoef, C., Vincent, M. \& de Weme, H. 2008. Focus op IT-bestuur: essentiële elementen van IT governance, The Hague, The Netherlands, SDU, Academic Service. 
Smits, D. \& van Hillegersberg, J. 2013. The continuing mismatch between IT governance theory and practice: Results from a Delphi study with CIO's. 19th Americas Conference on Information Systems (AMCIS), Chicago, IL. AIS Electronic Library, pp 3278-3286.

Smits, D. \& van Hillegersberg, J. 2014a. The continuing mismatch between it governance theory and practice: Results from a systematic literature review and a delphi study with cio's. Journal of management systems, Vol. 24, No. 3, pp 1-20.

Smits, D. \& van Hillegersberg, J. 2014b. The development of an IT governance maturity model for hard and soft governance. 8th European Conference on IS Management and Evaluation (ECIME), Brussels, Belgium. Academic Conferences and Publishing International Limited, pp 347-355.

Smits, D. \& van Hillegersberg, J. 2015. IT governance maturity: Developing a maturity model using the Delphi method. 48th Hawaii International Conference on System Sciences (HICSS), Kauai, Hawaii, USA. IEEE, pp 4534-4543.

Smits, D. \& van Hillegersberg, J. 2017. The development of a hard and soft IT governance assessment instrument. International Conference on ENTERprise Information Systems (CENTERIS), Barcelona, Spain. Elsevier, pp 47-54.

Smits, D. \& van Hillegersberg, J. 2018a. The continuing mismatch between IT governance maturity theory and practice: a new approach. International Conference on ENTERprise Information Systems (CENTERIS), Lisbon, Portugal. Elsevier, pp 549-560.

Smits, D. \& van Hillegersberg, J. 2018b. Hard and soft governance: The missing link between corporate and IT governance. Proceedings of the 6th International Conference on Management, Leadership and Governance (ICMLG), Bangkok, Thailand. Academic Conferences Limited, pp 421-430.

Spilter. 2014. Spilter [Online]. Available: https://www.spilter.nl/ [Accessed 11-7-2014 2014].

Spira, L. F. 1999. Ceremonies of governance: Perspectives on the role of the audit committee. Journal of Management and Governance, Vol. 3, No. 3, pp 231-260.

Spitzeck, H. \& Hansen, E. G. 2010. Stakeholder governance: How stakeholders influence corporate decision making. Corporate Governance, Vol. 10, No. 4, pp 378-391.

Spremić, M. 2012. Measuring IT governance performance: A research study on CobiT-based regulation framework usage. International Journal of Mathematics and Computers in Simulation, Vol. 1, No. 6, pp 17-25.

Straub, D. W. 1989. Validating instruments in MIS research. MIS Quarterly, Vol. 13, No. 2, pp 147-169.

Sundaramurthy, C. \& Lewis, M. 2003. Control and collaboration: Paradoxes of governance. Academy of Management Review, Vol. 28, No. 3, pp 397-415.

Surya, G. S. F. \& Surendro, K. 2014. E-readiness framework for cloud computing adoption in higher education. International Conference of Advanced Informatics: Concept, Theory and Application (ICAICTA), Bandung, Indonesia. IEEE, pp 278-282.

Tallon, P. P. \& Pinsonneault, A. 2011. Competing perspectives on the link between strategic information technology alignment and organizational agility: insights from a mediation model. MIS Quarterly, Vol. 35, No. 2, pp 463-486.

Tambotoh, J. J. \& LATUPERISSA, R. 2015. IT governance self assessment application for e-government implementation in Indonesia. Journal of Theoretical \& Applied Information Technology, Vol. 71, No. 1, pp 122-128.

Tanriverdi, H. 2006. Performance effects of information technology synergies in multibusiness firms. MIS Quarterly: Management Information Systems, Vol. 30, No. 1, pp 57-77. 


\section{References}

Tarhan, A., Turetken, O. \& Reijers, H. A. 2016. Business process maturity models: A systematic literature review. Information and Software Technology, Vol. 75, No., pp 122-134.

Tarmidi, M., Abdul Rashid, A. \& Abdul Roni, R. 2012. Exploring the approaches for COBIT process in Malaysian 100 top corporate governance companies. 3rd International Conference on Business and Economic Research (ICBER), Bandung, Indonesia.

Tavakolian, H. 1989. Linking the information technology structure with organizational competitive strategy: A survey. MIS Quarterly

Vol. 13, No. 3, pp 309-317.

Taylor-Powell, E. 2002. Quick tips collecting group data: Delphi technique. Madison, WI: University of Wisconsin.

Tirole, J. 2001. Corporate governance. Econometrica, Vol. 69, No. 1, pp 1-35.

Toms, S. \& Filatotchev, I. 2004. Corporate governance, business strategy, and the dynamics of networks: A theoretical model and application to the British cotton industry 1830-1980. Organization Studies, Vol. 25, No. 4, pp 629-651.

Trenholm, S. \& Jensen, A. 2000. Interpersonal communication, Belmont, CA, Wadsworth Publishing Company.

Tucker, C. M. 2003. The Lisbon strategy and the open method of coordination: a new vision and the revolutionary potential of soft governance in the European Union. Annual Meeting of the American Political Science Association, Philadelphia, Pennsylvania. pp 28-31.

Tugas, F. C. 2010. Information technology maturity index and profitability in yhe Philippine food, beverage and tobacco industry. International Journal of Business Research, Vol. 10, No. 1, pp 186-190.

Tuggle, C. S., Sirmon, D. G., Reutzel, C. R. \& Bierman, L. 2010. Commanding board of director attention: Investigating how organizational performance and CEO duality affect board members' attention to monitoring. Strategic Management Journal, Vol. 31, No. 9, pp 946-968.

Uehara, K. 2010. Soft IT Governance. ISACA Journal online, Vol. 1, No. 1, pp 1-6.

Uldam, J. \& Hansen, H. K. 2017. Corporate responses to stakeholder activism: partnerships and surveillance. Critical Perspectives on International Business, Vol. 13, No. 2, pp 151-165.

Ulrich, D. \& Smallwood, N. 2004. Capitalizing on capabilities. Harvard Business Review, Vol. 06, No. 1, pp 119-128.

Van Assche, K. \& Verschraegen, G. 2008. The limits of planning: Niklas Luhmann's systems theory and the analysis of planning and planning ambitions. Planning Theory, Vol. 7, No. 3, pp 263-283.

Van Grembergen, W. 2004. Strategies for information technology governance, Hershey, PA, USA, IGI Global.

Van Grembergen, W. \& De Haes, S. 2010. A research journey into enterprise governance of IT, business/ IT alignment and value creation. International Journal of IT/Business Alignment and Governance (IJITBAG), Vol. 1, No. 1, pp 1-13.

Van Grembergen, W., De Haes, S. \& Guldentops, E. 2004. Structures, processes and relational mechanisms for IT governance. Strategies for Information Technology Governance, Vol. 2, No. 004, pp 1-36.

Van Looy, A. 2014. Business process maturity: A comparative study on a sample of business process maturity models, Springer Science \& Business Media.

van Steenbergen, M., Bos, R., Brinkkemper, S., van de Weerd, I. \& Bekkers, W. 2010. The design of focus area maturity models. Global Perspectives on Design Science Research. Springer.

van Steenbergen, M., van den Berg, M. \& Brinkkemper, S. 2009. A balanced approach to developing the enterprise architecture practice. Enterprise Information Systems. Springer. 
Vejseli, S. \& Rossmann, A. 2017. The impact of IT governance on firm performance: A literature review. Pacific Asia Conference on Information Systems (PACIS), Langkawi, Malaysia.

Venable, J., Pries-Heje, J. \& Baskerville, R. 2012. A comprehensive framework for evaluation in design science research. International Conference on Design Science Research in Information Systems. Springer, pp 423-438.

Venable, J., Pries-Heje, J. \& Baskerville, R. 2016. FEDS: A framework for evaluation in design science research. European Journal of Information Systems, Vol. 25, No. 1, pp 77-89.

Verburgh, A., Elen, J. \& Lindblom-Ylänne, S. 2007. Investigating the myth of the relationship between teaching and research in higher education: A review of empirical research. Studies in Philosophy and Education, Vol. 26, No. 5, pp 449-465.

Verhezen, P. 2010. Giving voice in a culture of silence. From a culture of compliance to a culture of integrity. Journal of Business Ethics, Vol. 96, No. 2, pp 187-206.

Verhoef, C. 2007. Quantifying the effects of IT-governance rules. Science of Computer Programming, Vol. 67, No. 2, pp 247-277.

Von Solms, B. 2001. Information security - A multidimensional discipline. Computers and Security, Vol. 20, No. 6, pp 504-508.

Vugec, D. S., Spremić, M. \& Bach, M. P. 2017. IT governance adoption in banking and insurance sector: longitudinal case study of cobit use. International Journal for Quality Research, Vol. 11, No. 3.

Wahab, I. H. A. \& Arief, A. 2015. An integrative framework of COBIT and TOGAF for designing IT governance in local government. 2nd International Conference on Information Technology, Computer, and Electrical Engineering (ICITACEE). IEEE, pp 36-40.

Webb, P., Pollard, C. \& Ridley, G. 2006. Attempting to define IT governance: Wisdom or folly? 39th Annual Hawaii International Conference on Information Systems (HICCS). IEEE, pp 194a-194a.

Weber, C., Curtis, B. \& Gardiner, T. 2008. Business process maturity model (BPMM) version 1.0. Object Management Group (OMG).

Weill, P. \& Ross, J. 2005. A matrixed approach to designing IT governance. MIT Sloan Management Review, Vol. 46, No. 2, pp 26-34.

Weill, P. \& Ross, J. W. 2004. IT governance: How top performers manage IT decision rights for superior results, Boston, MA, Harvard Business Press.

Weill, P. \& Woodham, R. 2002. Don't just lead, govern: Implementing effective IT governance. Massachusetts, USA: Massachusetts Institute of Technology, USA.

Wendler, R. 2012. The maturity of maturity model research: A systematic mapping study. Information and Software Technology, Vol. 54, No. 12, pp 1317-1339.

Wernerfelt, B. 1984. A resource-based view of the firm. Strategic Management Journal, Vol. 5, No. 2, pp 171-180.

Westphal, J. D. \& Khanna, P. 2003. Keeping directors in line: Social distancing as a control mechanism in the corporate elite. Administrative Science Quarterly, Vol. 48, No. 3, pp 361-398.

Wieland, J. 2005. Corporate governance, values management, and standards: a European perspective. Business \& Society, Vol. 44, No. 1, pp 74-93.

Wijayanti, N., Setiawan, W. \& Sukamto, R. 2017. Performance assessment of IT governance with balanced scorecard and COBIT 4.1 of universitas Pendidikan Indonesia. Journal of Physics: Conference Seres, Bandung, Indonesia. IOP Publishing, pp 012072. 


\section{References}

Willcoxson, L., Manning, M. L., Johnston, N. \& Gething, K. 2011. Enhancing the research-teaching nexus: Building teaching-based research from research-based teaching. International Journal of Teaching and Learning in Higher Education, Vol. 23, No. 1, pp 1-10.

Williamson, O. E. 1999. Strategy research: Governance and competence perspectives. Strategic management journal, Vol. 20, No. 12, pp 1087-1108.

Willson, P. \& Pollard, C. 2009. Exploring IT governance in theory and practice in a large multi-national organisation in Australia. Information Systems Management, Vol. 26, No. 2, pp 98-109.

Wiseman, R. M. \& Gomez-Mejia, L. R. 1998. A behavioral agency model of managerial risk taking. Academy of Management Review, Vol. 23, No. 1, pp 133-153.

Wittenburg, A., Matthes, F., Fischer, F. \& Hallermeier, T. 2007. Building an integrated IT governance platform at the BMW Group. International Journal of Business Process Integration and Management, Vol. 2, No. 4, pp 327-337.

Wu, S. P.-J., Straub, D. W. \& Liang, T.-P. 2015. How information technology governance mechanisms and strategic alignment influence organizational performance: Insights from a matched survey of business and IT managers. MIS Quarterly, Vol. 39, No. 2, pp 497-518.

Xie, W. S. \& Li, C. 2011. Approaches to credit risk management in private banks: A case study of private bank in Taizhou city. 2011 International Conference on Electronics, Communications and Control, ICECC 2011 - Proceedings.

Xue, Y., Liang, H. \& Boulton, W. R. 2008. Information technology governance in information technology investment decision processes: The impact of investment characteristics, external environment, and internal context. MIS Quarterly: Management Information Systems, Vol. 32, No. 1, pp 67-96.

Yaokumah, W., Brown, S. \& Adjei, P. O.-M. 2015. Information technology governance barriers, Drivers, IT/ business alignment, and maturity in Ghanaian universities. International Journal of Information Systems in the Service Sector (IJISSS), Vol. 7, No. 4, pp 66-83.

Yin, R. K. 2013. Case study research: Design and methods, London, UK, Sage publications.

Yuwono, B. \& Vijaya, A. 2011. The impact of Information Technology Governance maturity level on corporate productivity: A case study at an Information Technology services company (WISSEN). Advanced Computer Science and Information System (ICACSIS), 2011 International Conference on. IEEE, pp 291-296.

Zelkowitz, M. V. \& Wallace, D. R. 1998. Experimental models for validating technology. Computer, Vol. 31, No. 5, pp 23-31.

Zhong, X., Vatanasakdakul, S. \& Aoun, C. 2012. IT governance In China: Cultural fit and IT governance capabilities. 16th Pacific Asia Conference on Information Systems (PACIS), Ho Chi Minh City, Vietnam. pp 55.

Zhu, D. \& Li, F. 2014. The IT governance: Operating model and governance framework: Methodology and practice in group-wide corporation. International Conference on Management of e-Commerce and e-Government (ICMeCG), Shanghai, China. IEEE, pp 291-294. 


\section{PUBLICATION L IS T}

a. Smits, D. \& van Hillegersberg, J. 2013. The continuing mismatch between IT governance theory and practice: Results from a Delphi study with CIO's. 19th Americas Conference on Information Systems (AMCIS), Chicago, IL. AIS Electronic Library, pp 3278-3286.

b. Smits, D. \& van Hillegersberg, J. 2014a. The continuing mismatch between it governance theory and practice: Results from a systematic literature review and a delphi study with cio's. Journal of management systems, Vol. 24, No. 3, pp 1-20.

c. Smits, D. \& van Hillegersberg, J. 2014b. The development of an IT governance maturity model for hard and soft governance. 8th European Conference on IS Management and Evaluation (ECIME), Brussels, Belgium. Academic Conferences and Publishing International Limited, pp 347-355.

d. Smits, D. \& van Hillegersberg, J. 2015. IT governance maturity: Developing a maturity model using the Delphi method. 48th Hawaii International Conference on System Sciences (HICSS), Kauai, Hawaii, USA. IEEE, pp 4534-4543.

e. Smits, D. \& van Hillegersberg, J. 2017. The development of a hard and soft IT governance assessment instrument. International Conference on ENTERprise Information Systems (CENTERIS), Barcelona, Spain. Elsevier, pp 47-54.

f. Smits, D. \& van Hillegersberg, J. 2018a. The continuing mismatch between IT governance maturity theory and practice: a new approach. International Conference on ENTERprise Information Systems (CENTERIS), Lisbon, Portugal. Elsevier, pp 549-560.

g. Smits, D. \& van Hillegersberg, J. 2018b. Hard and soft governance: The missing link between corporate and IT governance. Proceedings of the 6th International Conference on Management, Leadership and Governance (ICMLG), Bangkok, Thailand. Academic Conferences Limited, pp 421-430. 


\section{APPENDIX A LIST OF FIGURES}

$\begin{array}{llr}\text { Figure } 1 & \text { Thesis Part I. Introduction, motivation and research description } & 10\end{array}$

Figure 2 Relationship between ITG and firm performance 14

$\begin{array}{lll}\text { Figure } 3 & \text { Research model } & 32\end{array}$

Figure $4 \quad$ Research model including research questions $\quad 32$

Figure $5 \quad$ Structure of the thesis 33

$\begin{array}{lll}\text { Figure } 6 & \text { Research outline: activities and results } & 39\end{array}$

Figure $7 \quad$ Thesis Part II. Collect prior knowledge and assess practical relevance 42

Figure $8 \quad$ Activities and results of Step II, Part A. 43

$\begin{array}{lll}\text { Figure } 9 & \text { Activities of Step II, Part B. } & 61\end{array}$

Figure $10 \quad$ Thesis Part III. Design and evaluation of the model and the instrument 71

$\begin{array}{lll}\text { Figure } 11 & \text { Activities and results of Step III, Part A. } & 72\end{array}$

$\begin{array}{lll}\text { Figure } 12 & \text { Design process of the MIG model } & 74\end{array}$

$\begin{array}{lll}\text { Figure } 13 \quad \text { Example screenshot from Spilter } & 75\end{array}$

$\begin{array}{lll}\text { Figure } 14 \quad \text { Change of the basic model } & 81\end{array}$

$\begin{array}{lll}\text { Figure } 15 & \text { Focus area maturity model } & 87\end{array}$

Figure 16 The design of focus area maturity models, adopted from van Steenbergen et al. (2010) 88

$\begin{array}{lll}\text { Figure } 17 & \text { Activities and results of Step III, Part B. } & 107\end{array}$

$\begin{array}{llr}\text { Figure } 18 & \text { Case study protocol for the MIG assessment } & 109\end{array}$

Figure 19 Examples of statements for focus area "Functions and roles" in questionnaire 1 (v. 1) 113

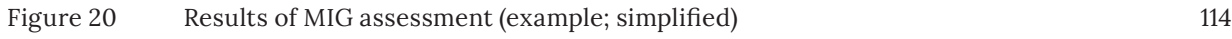

Figure $21 \quad$ Example of the statements for informal organisation (simplified version) 118

Figure 22 Thesis Part IV. Use, improve and validate the instrument 120

$\begin{array}{lll}\text { Figure } 23 & \text { Activities and results of Step IV, Part A. } & 121\end{array}$

Figure 24 Example results sheet MIG assessment instrument v. 3, the entire organisation 134

$\begin{array}{lll}\text { Figure 25 } & \text { Activities and results of Step IV, Part B. } & 147\end{array}$

Figure $26 \quad$ Corporate governance, IT governance and the context. 154

$\begin{array}{lll}\text { Figure } 27 & \text { Thesis Part V. Conclusions, limitations and future research } & 157\end{array}$

Figure 28 Corporate governance, IT governance and the context. (Figure 26 and Figure 28 are equal) 164

$\begin{array}{llr}\text { Figure } 29 \quad \text { Name and Organization, MIG assessment v. } 3 & 191\end{array}$

Figure $30 \quad$ Example results sheet MIG assessment instrument v. 3, my department 199

Figure $31 \quad$ Example results sheet MIG assessment instrument v. 3, the entire organisation 200

Figure 32 Competing Values Framework, adopted from Quinn and Rohrbaugh (1983) 204 


\section{APPENDIX B LIST OF TABLES}

Table

Six ITG streams

Table $2 \quad$ Use of ITG frameworks (ITGI, 2008, 2011)

Table 3

Dimensions of ITG (Novotny et al., 2012)

Table 4

Relevant focus areas for IT governance

Table 5

Link between the four validity and reliability criteria and the design cycle

Table 6

Research questions and sub-questions per step

Table 7

Table 8

Research approach, including parties involved in the activities.

Table 9

Totals systematic literature review 2012/2013

Table 10

Documents on ITG grouped by specific topics

Table 11

Year of publication of the documents

Table 12

Six ITG streams (Table 1 and Table 12 are equal)

Table 13

Meeting attendance

Table 14

Reporting line

Table 15

Views regarding ITG implementation (regarding scope)

Table 16

Working direction of ITG

Table 17

Important disciplines for ITG according to the CIOs

Table 18

Actions to improve ITG

Table 19

Totals systematic literature review

Table 20

Year of publication of the documents 


\section{Appendix B}

Table 33

Table 34

Table 35

Table 36

Table 37

Table 38

Table 39

Table 40

Table 41

Table 42

Table 43

Table 44

Table 45

Table 46

Table 47

Table 48

Table 49

Table 50

Table 51

Table 52

Table 53

Table 54

Table 55

Table 56

Table 57

Table 58

Table 59

Table 60

Table 61

Table 62

Table 63

Table 64

Table 65

Table 66
Selected sources (end result)

Focus area: Continuous improvement

Focus area: Leadership

Focus area: Participation

Focus area: Understanding and trust

Capabilities of the maturity model (end result)

Selected sources (end result)

Focus area: Culture

Definition of the domains (version 1.0)

Definition of the focus areas (version 1.0)

The MIG model version 1.0 (end result)

Support for key elements MIG assessment instrument

Conceptual framework of the MIG assessment instrument: nine focus areas

Conceptual framework of the MIG assessment instrument: the informal organisation

117

Evaluation of the MIG model and the MIG assessment instrument based on Gregor et al.123

Overview of case studies in 2015

Hard and soft IT governance results before and after the interviews (before; after)

Results of IT governance context before and after the interviews

Selected changes for the MIG assessment instrument after the first cycle

Overview of case studies in 2016

Evaluation of the changes in 2016

Selected changes for the MIG assessment instrument after the second cycle

Overview of case studies in 2017 (after the interviews)

Results of the hard and soft governance, department view (before; after)

Results of the context, view: value chain, after the interview 
Table 67 Overview of case studies (after the interview) 140

Table 68 Evaluation of the changes in 2017

Table 69 Summary of the evaluation

Table $70 \quad$ Summary of the KNVI evaluation (complete report)

Table 71 Documents in Scopus on corporate governance grouped by specific topics 150

Table 72 Selected corporate governance papers per focus area or topic 152

Table 73 MIG assessment instrument: list of definitions (v. 3) 194

Table 74 MIG assessment instrument v. 3, Questionnaire 1: the MIG model 197

Table 75 MIG assessment instrument v. 3, Questionnaire 2: the informal organisation 197

Table 76 Legend for Appendix F.1, F.2 and F.3 (extract case study database)

Table 77 Results case study 1-5 in 2015 (end result) 206

Table 78 Results case study 6-10 in 2015 (end result) 207

Table 79 Results case study 1-10 in 2016, hard and soft governance (end result)

Table 80 Results case study 1-10 in 2016, context (end result)

Table 81 Results case studies 2017, hard and soft governance (department view, end result)

Table 82 Results case studies 2017, context (department view, end result)

Table 83 Results case studies 2017, hard and soft governance (organisation view, end result) 


\section{APPENDIX C THE MIG ASSESSMENT INSTRUMENT V. 3}

This appendix describes the customer version of version 3 of the MIG assessment instrument ${ }^{15}$. It consists of an Excel sheet containing five tabs:

- $\quad$ Name and Organisation (C1);

- $\quad$ Introduction and Explanation (C2);

- $\quad$ List of definitions (C3);

- $\quad$ Questionnaire 1: The MIG model (C4);

- $\quad$ Questionnaire 2: Informal organisation (C5);

- $\quad$ Questionnaire 3: Culture (C6).

The statements of questionnaire 1 and 2 of the MIG assessment instrument (version 3) are listed. In the actual instrument the statements of questionnaire 1 are presented in a random order, but are grouped by focus area. Questionnaire 3 is based on the Organizational Cultural Assessment Instrument (OCAI).

A hidden data tab is used to export the data to the researcher version of the instrument. This version can be used to generate the reports shown in the last two sections of this appendix:

- $\quad$ Results MIG assessment; results for My department (C7).

- $\quad$ Results MIG assessment; results for The entire organisation (C8).

The MIG assessment instrument is described in detail in Section 6.

15 The first, second and third version of the MIG assessment instrument were originally written in American English. 


\section{C.1 Name and Organisation}

MIG version 3

UNIVERSITY OF TWENTE.

MIG assessment

The Tab "Introduction and Explanation" contains a short definition of the terminology used.

\begin{tabular}{|l|l|}
\hline Name & \multicolumn{2}{|l|}{} \\
\hline Function & \multicolumn{2}{|l|}{} \\
\hline Organization & \\
\hline My department & \\
\hline $\begin{array}{l}\text { I am the manager of this } \\
\text { department (Yes/No) }\end{array}$ & \\
\hline Next level & \\
\hline Date of the assessment & \\
\hline
\end{tabular}

\begin{tabular}{|l|l|}
\hline E-mail: & \\
\hline
\end{tabular}

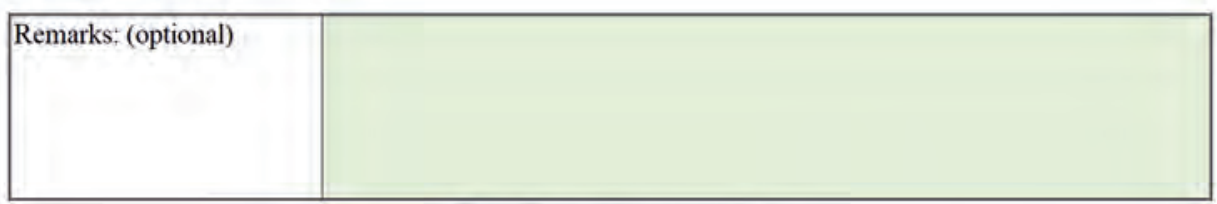

Data will be used in our research.

All data will be kept confidential.

Please leave your e-mail address if you would like to be informed of the results of the research.

If there are questions about our research, we can be contacted at: d.smits@utwente.nl

Figure 29 Name and Organization, MIG assessment v. 3 


\section{2 Introduction and Explanation}

Introduction and Explanation

The MIG assessment is an assessment using the MIG model. The MIG model is a maturity model for hard and soft IT governance. Hard governance is focused on processes and structures; soft governance on areas such as behaviour or organisational culture. This assessment is intended to ascertain an impression of how the organisation works from both perspectives. The MIG assessment is an instrument of ongoing research. This document contains version 3 of the instrument.

\section{Answering the questionnaire}

Questions should be entered twice:

- $\quad$ Once for "My department", meaning the department in which you are working or the department you are leading;

- Once for "The entire organisation".

This aims to make it easier to answer the questions for organisations in which the maturity level differs between departments (this applies to most contemporary organisations). In the assessments, both views have been placed next to each other in separate columns.

If the questionnaire is taking too much time

If possible, we would appreciate it if all answers could be entered twice. However, we can also use data for only one view. If you are short of time or are unwilling to answer questions twice, please choose one of the views in each assessment. For us to be able to use the data it is important that you choose the same view in each assessment.

The MIG assessment consists of three questionnaires:

\section{Assessment "1 The MIG model"}

Decide to what extent you agree with 70 statements.

In assessment 1 we use a scale of six levels meaning:

- $\quad$ Strongly disagree (0\%);

- $\quad$ Disagree (20\% or less);

- $\quad$ Somewhat disagree (40\%);

- $\quad$ Somewhat agree (60\%);

- $\quad$ Agree (80\% or more);

- $\quad$ Strongly agree (100\%). 
Appendix C

In the maturity model, only "Largely agree" and "Completely agree" will result in reaching a maturity level!

2. Assessment 2 "Informal organisation"

Divide points between nine groups of two statements.

\section{Assessment 3 "Culture"}

Divide points between six groups of four statements. 


\section{3 List of definitions}

A list of definitions for the elements used in this assessment:

\begin{tabular}{|c|c|}
\hline Element & Definition \\
\hline Behaviour & $\begin{array}{l}\text { Anything that an organism does involving action and response to stimulation; } \\
\text { the response of an individual, group or species to its environment; the way in } \\
\text { which something functions or operates. }\end{array}$ \\
\hline Collaboration & Collaboration is defined as making a joint effort towards a goal. \\
\hline $\begin{array}{l}\text { Continuous } \\
\text { improvement }\end{array}$ & $\begin{array}{l}\text { A continual stream of innovation in order to enhance the value or quality of } \\
\text { the products and processes of an organisation. }\end{array}$ \\
\hline Culture & $\begin{array}{l}\text { The beliefs, values and norms of the members of the organisation that define } \\
\text { the way they interact. The entire organisation, not just IT. }\end{array}$ \\
\hline Formal networks & The formal governing bodies that are part of the organisation. \\
\hline Functions and roles & $\begin{array}{l}\text { The organisational hierarchal and non-hierarchal positions as defined in the } \\
\text { organisation. }\end{array}$ \\
\hline Hard governance & $\begin{array}{l}\text { The hard side of ITG involves the functional aspects of governance such as } \\
\text { structures and processes. }\end{array}$ \\
\hline $\begin{array}{l}\text { Informal } \\
\text { organisation }\end{array}$ & $\begin{array}{l}\text { The emergent pattern of social interactions within the organisation that } \\
\text { emerge rather than are mandated. The entire organisation, not just IT. }\end{array}$ \\
\hline IT decision-making & $\begin{array}{l}\text { IT-related decision-making processes, decision rights and the accountability } \\
\text { framework. }\end{array}$ \\
\hline Leadership & $\begin{array}{l}\text { Behaviour that results in the supervision, organisation and change of the lives, } \\
\text { perceptions, expectations and values of the members of an organisation. }\end{array}$ \\
\hline Monitoring & $\begin{array}{l}\text { The monitoring of the costs, values and risks of the continuation and change } \\
\text { of the IT services in an organisation. }\end{array}$ \\
\hline Participation & $\begin{array}{l}\text { Having a part or share in the interaction between the stakeholders in an } \\
\text { organisation. }\end{array}$ \\
\hline Planning & $\begin{array}{l}\text { The establishment of goals, policies and procedures for a social or economic } \\
\text { unit. In this model, this is seen as a top-down and bottom-up process. }\end{array}$ \\
\hline Process & $\begin{array}{l}\text { Formalisation and institutionalisation of strategic IT decision-making or IT } \\
\text { monitoring procedures. }\end{array}$ \\
\hline Sector & A sociological, economic or political subdivision of society. \\
\hline Soft governance & $\begin{array}{l}\text { The soft side of ITG is defined as relating to social aspects such as human } \\
\text { behaviour and organisational culture. }\end{array}$ \\
\hline Structure & $\begin{array}{l}\text { Structural (formal) devices and mechanisms for connecting and enabling } \\
\text { horizontal contacts or liaisons between business and IT management } \\
\text { (decision-making) functions. }\end{array}$ \\
\hline $\begin{array}{l}\text { Understanding and } \\
\text { trust }\end{array}$ & $\begin{array}{l}\text { Shared knowledge and confidence between the stakeholders in an } \\
\text { organisation. }\end{array}$ \\
\hline
\end{tabular}

Table 73 MIG assessment instrument: list of definitions (v. 3) 


\section{C.4 Questionnaire 1: the MIG model}

Column Id refers to the maturity level. Each level is represented by two statements. Within a focus area, higher numbers represent a higher maturity level. To prevent participants from taking this into account, the statements are sorted in a random order.

\begin{tabular}{|c|c|c|}
\hline Id & Focus area & Statement \\
\hline 1 & $\begin{array}{l}\text { Continuous } \\
\text { improvement }\end{array}$ & There is a structure for improving the organisation continuously. \\
\hline 2 & $\begin{array}{l}\text { Continuous } \\
\text { improvement }\end{array}$ & There is a system for improving the organisation continuously. \\
\hline 3 & $\begin{array}{l}\text { Continuous } \\
\text { improvement }\end{array}$ & The continuous improvement of our organisation is goal-oriented. \\
\hline 4 & $\begin{array}{l}\text { Continuous } \\
\text { improvement }\end{array}$ & The continuous improvement of our organisation is linked to strategy. \\
\hline 5 & $\begin{array}{l}\text { Continuous } \\
\text { improvement }\end{array}$ & Our organisation is proactively and continuously improved. \\
\hline 6 & $\begin{array}{l}\text { Continuous } \\
\text { improvement }\end{array}$ & Employees are empowered to improve the organisation continuously. \\
\hline 7 & $\begin{array}{l}\text { Continuous } \\
\text { improvement }\end{array}$ & Our organisation has a learning mechanism for continuous improvement. \\
\hline 8 & $\begin{array}{l}\text { Continuous } \\
\text { improvement }\end{array}$ & $\begin{array}{l}\text { Our organisation has an 'unlearning' mechanism for continuous } \\
\text { improvement. }\end{array}$ \\
\hline 9 & Leadership & My manager/CEO contributes with knowledge. \\
\hline 10 & Leadership & My manager/CEO has good work habits. \\
\hline 11 & Leadership & My manager/CEO contributes to the group objectives. \\
\hline 12 & Leadership & My manager/CEO works effectively in groups. \\
\hline 13 & Leadership & My manager/CEO organises effectively to reach the group objectives. \\
\hline 14 & Leadership & My manager/CEO is competent. \\
\hline 15 & Leadership & My manager/CEO catalyses commitment. \\
\hline 16 & Leadership & My manager/CEO encourages high performance standards. \\
\hline 17 & Leadership & My manager/CEO is humble. \\
\hline 18 & Leadership & My manager/CEO is willful. \\
\hline 19 & Participation & $\begin{array}{l}\text { Group members need the role of the coordinator to centralise and manage } \\
\text { activities. }\end{array}$ \\
\hline 20 & Participation & Business processes include basic collaboration processes. \\
\hline 21 & Participation & Group members work in a self-organised way to achieve group goals. \\
\hline 22 & Participation & Collaboration processes include monitoring and controlling. \\
\hline 23 & Participation & Group members share results and knowledge inside the organisation. \\
\hline 24 & Participation & Collaboration processes are formally structured. \\
\hline
\end{tabular}




\begin{tabular}{|c|c|c|}
\hline Id & Focus area & Statement \\
\hline 25 & $\begin{array}{l}\text { Understanding } \\
\text { and trust }\end{array}$ & Employees have a general understanding of each other's current objectives. \\
\hline 26 & $\begin{array}{l}\text { Understanding } \\
\text { and trust }\end{array}$ & Employees have a general understanding of each other's vision for IT. \\
\hline 27 & $\begin{array}{l}\text { Understanding } \\
\text { and trust }\end{array}$ & Employees understand each other's current high-priority objectives. \\
\hline 28 & $\begin{array}{l}\text { Understanding } \\
\text { and trust }\end{array}$ & Employees understand each other's vision for IT. \\
\hline 29 & $\begin{array}{l}\text { Understanding } \\
\text { and trust }\end{array}$ & Employees are committed to each other's current objectives. \\
\hline 30 & $\begin{array}{l}\text { Understanding } \\
\text { and trust }\end{array}$ & Employees have a shared vision for IT. \\
\hline 31 & $\begin{array}{l}\text { Functions and } \\
\text { roles }\end{array}$ & Functions and roles in processes are repeatable. \\
\hline 32 & $\begin{array}{l}\text { Functions and } \\
\text { roles }\end{array}$ & Functions and roles are based on policy. \\
\hline 33 & $\begin{array}{l}\text { Functions and } \\
\text { roles }\end{array}$ & Functions and roles are defined. \\
\hline 34 & $\begin{array}{l}\text { Functions and } \\
\text { roles }\end{array}$ & Functions and roles are standardised. \\
\hline 35 & $\begin{array}{l}\text { Functions and } \\
\text { roles }\end{array}$ & Functions and roles are managed. \\
\hline 36 & $\begin{array}{l}\text { Functions and } \\
\text { roles }\end{array}$ & $\begin{array}{l}\text { When we improve functions and roles we focus on improving process } \\
\text { performance. }\end{array}$ \\
\hline 37 & $\begin{array}{l}\text { Functions and } \\
\text { roles }\end{array}$ & Functions and roles are continually improved. \\
\hline 38 & $\begin{array}{l}\text { Functions and } \\
\text { roles }\end{array}$ & $\begin{array}{l}\text { When we improve functions and roles we focus on improving organisational } \\
\text { performance. }\end{array}$ \\
\hline 39 & Formal Networks & Formal networks in processes are repeatable. \\
\hline 40 & Formal Networks & Formal networks are based on policy. \\
\hline 41 & Formal Networks & Formal networks in processes are defined. \\
\hline 42 & Formal Networks & Formal networks are standardised. \\
\hline 43 & Formal Networks & Formal networks are managed. \\
\hline 44 & Formal Networks & $\begin{array}{l}\text { When we improve formal networks we focus on improving process } \\
\text { performance. }\end{array}$ \\
\hline 45 & Formal Networks & Formal networks are continually improved. \\
\hline 46 & Formal Networks & $\begin{array}{l}\text { When we improve formal networks we focus on improving organisational } \\
\text { performance. }\end{array}$ \\
\hline 47 & $\begin{array}{l}\text { IT decision- } \\
\text { making }\end{array}$ & IT decision-making processes are repeatable. \\
\hline 48 & $\begin{array}{l}\text { IT decision- } \\
\text { making }\end{array}$ & IT decision-making processes are based on policy. \\
\hline
\end{tabular}




\begin{tabular}{|c|c|c|}
\hline Id & Focus area & Statement \\
\hline 49 & $\begin{array}{l}\text { IT decision- } \\
\text { making }\end{array}$ & IT decision-making processes are defined. \\
\hline 50 & $\begin{array}{l}\text { IT decision- } \\
\text { making }\end{array}$ & IT decision-making processes are standardised. \\
\hline 51 & $\begin{array}{l}\text { IT decision- } \\
\text { making }\end{array}$ & IT decision-making processes are managed. \\
\hline 52 & $\begin{array}{l}\text { IT decision- } \\
\text { making }\end{array}$ & $\begin{array}{l}\text { When we improve IT decision-making we focus on improving process } \\
\text { performance. }\end{array}$ \\
\hline 53 & $\begin{array}{l}\text { IT decision- } \\
\text { making }\end{array}$ & IT decision-making processes are continually improved. \\
\hline 54 & $\begin{array}{l}\text { IT decision- } \\
\text { making }\end{array}$ & $\begin{array}{l}\text { When we improve IT decision-making we focus on improving organisational } \\
\text { performance. }\end{array}$ \\
\hline 55 & Planning & Processes used for planning are repeatable. \\
\hline 56 & Planning & Processes used for planning are based on policy. \\
\hline 57 & Planning & Processes used for planning are defined. \\
\hline 58 & Planning & Processes used for planning are standardised. \\
\hline 59 & Planning & Processes used for planning are managed. \\
\hline 60 & Planning & When we improve planning we focus on improving process performance. \\
\hline 61 & Planning & Processes used for planning are continually improved. \\
\hline 62 & Planning & $\begin{array}{l}\text { When we improve planning we focus on improving organisational } \\
\text { performance. }\end{array}$ \\
\hline 63 & Monitoring & Processes used for monitoring are repeatable. \\
\hline 64 & Monitoring & Processes used for monitoring are based on policy. \\
\hline 65 & Monitoring & Processes used for monitoring are defined. \\
\hline 66 & Monitoring & Processes used for monitoring are standardised. \\
\hline 67 & Monitoring & Processes used for monitoring are managed. \\
\hline 68 & Monitoring & When we improve monitoring we focus on improving process performance. \\
\hline 69 & Monitoring & Processes used for monitoring are continually improved. \\
\hline 70 & Monitoring & $\begin{array}{l}\text { When we improve monitoring we focus on improving organisational } \\
\text { performance. }\end{array}$ \\
\hline
\end{tabular}

Table 74 MIG assessment instrument v. 3, Questionnaire 1: the MIG model 


\section{C.5 Questionnaire 2: the informal organisation}

In questionnaire 2 the participants have to divide 100 points between the formal and informal column for each of the nine focus areas of the MIG model.

\begin{tabular}{|c|l|l|}
\hline Id & Formal & Informal \\
\hline 1 & $\begin{array}{l}\text { Improvements are the result of bottom- } \\
\text { up initiatives. }\end{array}$ & Improvements are the result of management initiatives. \\
\hline 2 & Leadership is shown by non-managers. & Leadership is shown by managers. \\
\hline 3 & $\begin{array}{l}\text { Collaboration depends on informal } \\
\text { processes. }\end{array}$ & Collaboration depends on formal processes. \\
\hline 4 & $\begin{array}{l}\text { Employees (including managers) trust } \\
\text { informal leaders. }\end{array}$ & Employees (including managers) trust their manager. \\
\hline 5 & $\begin{array}{l}\text { Our organisation is based on informal } \\
\text { functions and roles. }\end{array}$ & Our organisation is based on formal functions and roles. \\
\hline 6 & $\begin{array}{l}\text { Our governance is based on informal } \\
\text { networks. }\end{array}$ & Our governance is based on formal networks. \\
\hline 7 & $\begin{array}{l}\text { IT decision-making is informally } \\
\text { organised. }\end{array}$ & IT decision-making is formally organised. \\
\hline 8 & We use informal planning. & We use formal planning. \\
\hline 9 & Monitoring is an informal process. & Monitoring is a formal process. \\
\hline
\end{tabular}

Table 75 MIG assessment instrument v. 3, Questionnaire 2: the informal organisation

\section{C.6 Questionnaire 3: culture}

Questionnaire 3 is not included as we are using an existing questionnaire: the Organizational Cultural Assessment Instrument (OCAI). The OCAI instrument was developed by Cameron and Quinn (2005). 
Appendix C

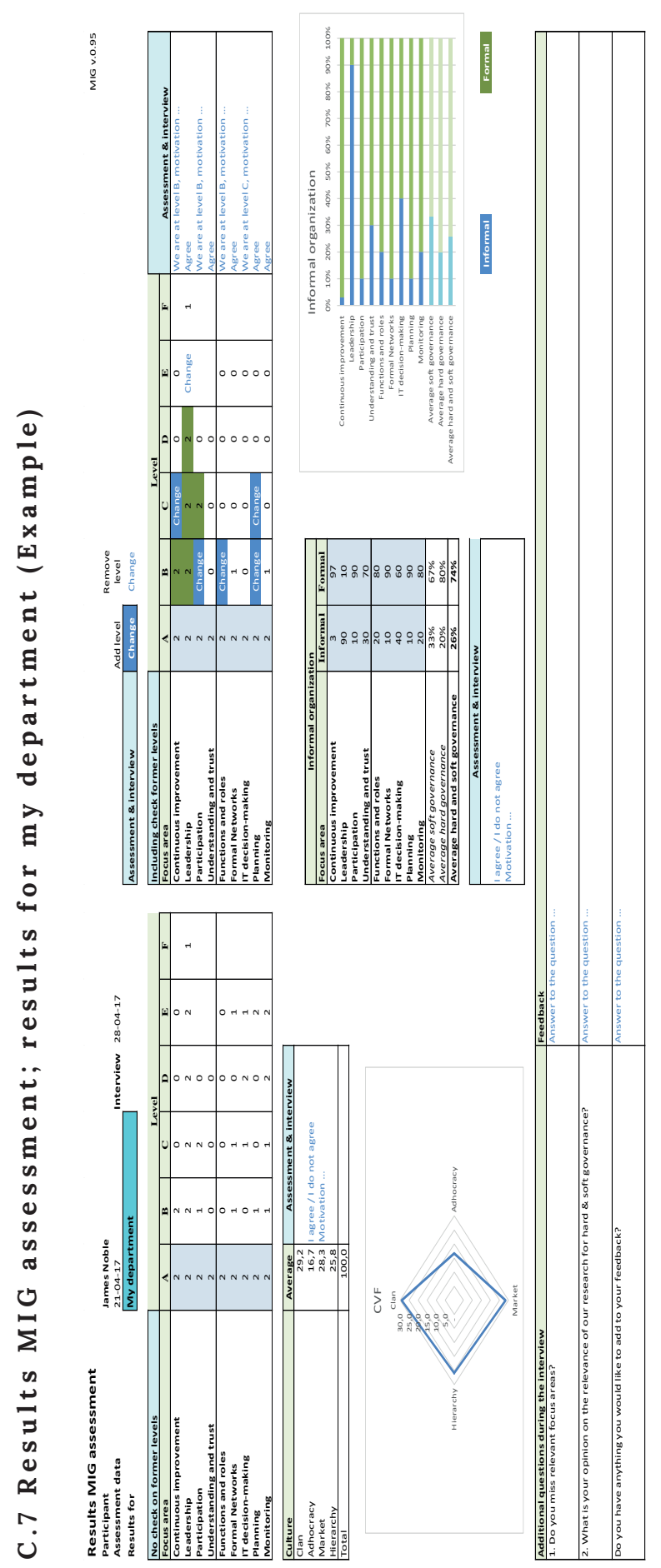

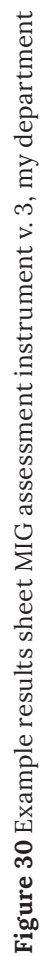


Appendix C

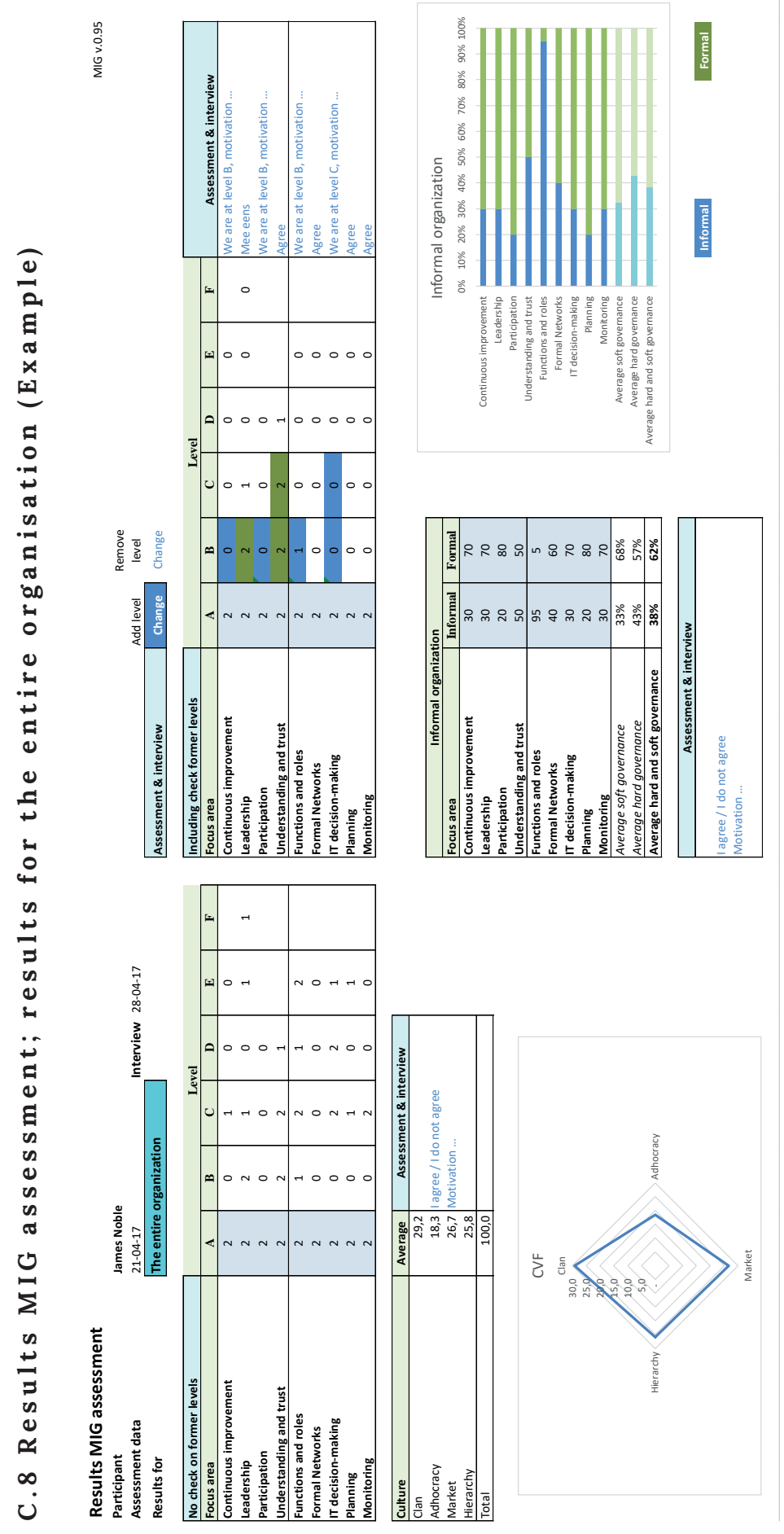

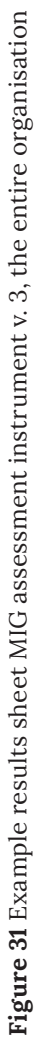




\section{APPENDIX D STRUCTURE MIG REPORT V. 2}

The appendix contains an overview of the structure of the MIG report v. 2

I. Introduction

This section is organised as follows:

- $\quad$ Description of the process selecting the organisation and the participants;

- $\quad$ Introduction to the research;

- $\quad$ Explanation of the MIG model (Table 49) and the MIG assessment instrument (Appendix C);

- $\quad$ Description of the approach of the case study;

- $\quad$ Definition of the department (first perspective) and the organisation (second perspective).

II. Summary, conclusion and recommendation

This section is organised as follows:

- $\quad$ Summary of the results (after the interviews);

- Conclusion;

- Recommendation.

III. Results

In the results section the results of the assessments, interviews and evaluations are discussed. In the discussion the corrected results after the interviews are used. The discussions are illustrated with anonymous citations from the interviews. This section is organised as follows:

- $\quad$ Detailed summarised and anonymous overview of:

- $\quad$ All results (department perspective; after the interviews);

- $\quad$ All results (organisation perspective; after the interviews);

- $\quad$ All results of the evaluation form.

- $\quad$ Short discussion of the results of each hard governance focus area;

- $\quad$ Short discussion of the results of each soft governance focus area;

- $\quad$ Short discussion of the results of each focus area of the context;

- Short discussion of the results of the evaluation. 


\section{Appendices}

This section includes (examples of) the handouts during the interviews:

- $\quad$ Example of the results sheets (see Appendix C.7 and C.8);

- $\quad$ Description of the maturity levels of the MIG model (see Appendix E). 


\section{APPENDIX E HANDOUT INTERVIEW MIG ASSESSMENT V. 3}

The appendix contains an overview of the handout used in the interviews with the participants of the case studies conducted by the researchers.

The first maturity level is A. This is the starting level.

\section{Soft Governance}

Soft governance consists of four elements:

- $\quad$ Continuous improvement;

- $\quad$ Leadership:

- Participation;

- $\quad$ Understanding and trust.

\section{Continuous improvement (CI)}

A. Improvement requires a trigger and implementation is on an ad hoc basis.

B. There is formal commitment to building a system.

C. CI behaviour is established at a level local to the wider strategic concerns of the organisation.

D. Devolve autonomy and empower individuals and groups.

E. Full CI capability approximates to a model "learning organisation". Systematic finding and solving problems and capturing and sharing learning.

\section{Le a d e rship}

A. Does not contribute.

B. Contributes through talent, knowledge, skills and good work habits.

C. Contributes to the achievement of group objectives.

D. Organises people and resources toward the effective and efficient pursuit of predetermined objectives.

E. Catalyses commitment to and vigorous pursuit of a clear and compelling vision. Personal humility and professional will.

F. Builds enduring greatness through a paradoxical combination of personal humility plus professional will. 


\section{Participation}

A. Collaboration is not explicitly represented in business processes. Collaboration is dependent on individual initiative and skills, and its success depends on relationships among people.

B. Business processes start to be modified with the aim of including basic collaboration activities. Coordination is an important aspect because groups need leadership and management in order to guide their work. The role of the coordinator is needed to centralise and to manage activities.

C. The process includes activities for monitoring and controlling how collaboration occurs. Group members are aware of their tasks and responsibilities and are committed towards them. Group members work in a self-organised and simultaneous way to achieve the group's goals.

D. Processes must be formally concluded and their results communicated. Workgroup results are published and shared inside the organisation. Process tacit knowledge is shared through ideas, opinions and experiences.

\section{Understanding and trust}

A. Members cannot identify each other's major current objectives or vision for IT.

B. Members have a general understanding of each other's current objectives and vision for IT.

C. Members have a specific understanding of each other's current objectives and vision for IT and can identify specific high-priority objectives.

D. Members understand and are committed to each other's current objectives and have a shared vision for IT.

\section{Hard Governance}

Hard governance consists of five elements:

- $\quad$ Functions and roles;

- $\quad$ Formal networks;

- $\quad$ IT decision-making;

- Planning;

- $\quad$ Monitoring. 
Hard governance is based on CMM. All focus areas in this domain use the CMM maturity levels.

A. Initial. Processes are usually ad hoc and chaotic.

B. Repeatable. Processes are planned in accordance with policy.

C. Defined. Defined processes are used for managing work.

D. Managed. Quantitative objectives are defined for quality and process performance and used as criteria in managing processes.

E. Optimising. An organisation continually improves its processes based on a quantitative understanding of its business objectives and performance needs.

\title{
Context
}

The context consists of three elements:

\author{
- $\quad$ Culture; \\ - Informal organisation; \\ - $\quad$ Sector.
}

\section{Culture}

Culture is based on the Competing Values Framework (CVF).

The framework has four quadrants:

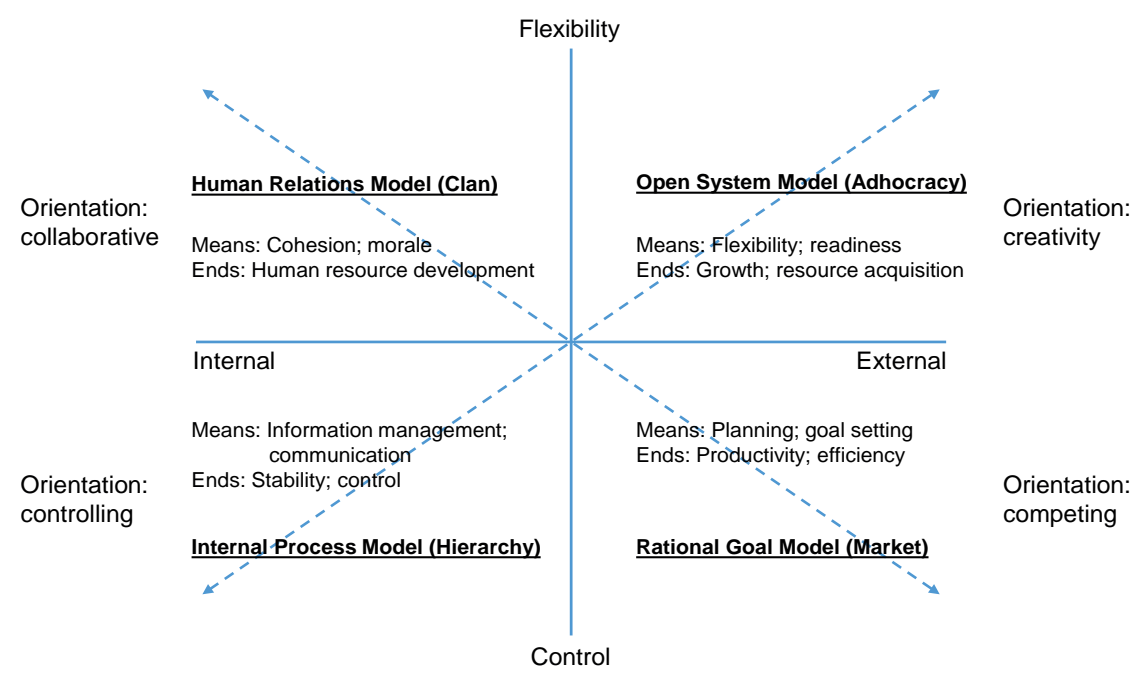

Figure 32 Competing Values Framework, adopted from Quinn and Rohrbaugh (1983) 


\section{APPENDIX F RESULTS CASE STUDIES BETWEEN 2015 AND 2017}

In this appendix the results of the case studies between 2015 and 2017 are summarised.

The tables summarise the end result of the case study as presented to:

- $\quad$ The customer (case studies conducted by the researchers); or

- $\quad$ The researchers (case studies conducted by the students).

F.1 Results case studies in 2015

F.2 Results case studies in 2016

F.3 Results case studies in 2017

\section{Le gend}

\begin{tabular}{|l|l|}
\hline Field & Description \\
\hline Case & Number of the case study \\
\hline \#P & Participant number \\
\hline CI & Continuous improvement \\
\hline Lead & Leadership \\
\hline Part & Participation \\
\hline Trst & Understanding and trust \\
\hline F\&R & Functions and roles \\
\hline FN & Formal networks \\
\hline Deci & IT decision-making \\
\hline Plan & Planning \\
\hline Mon & Monitoring \\
\hline Clan & Clan \\
\hline Adh & Adhocracy \\
\hline Market & Market \\
\hline Hierarch & Hierarchy \\
\hline Soft & Soft governance focus areas \\
\hline Hard & Hard governance focus areas \\
\hline Informal & Informal organisation (\%) \\
\hline
\end{tabular}

Table 76 Legend for Appendix F.1, F.2 and F.3 (extract case study database) 


\begin{tabular}{|c|c|c|c|c|c|c|c|c|c|c|c|c|c|c|c|c|}
\hline \multicolumn{3}{|c|}{ Extract from case study database v. 0.82} & \multicolumn{4}{|c|}{$\begin{array}{c}\text { Soft } \\
\text { governance }\end{array}$} & \multicolumn{5}{|c|}{$\begin{array}{c}\text { Hard } \\
\text { governance }\end{array}$} & \multicolumn{4}{|c|}{ Culture } & \multirow[b]{2}{*}{ 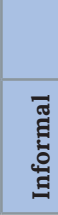 } \\
\hline Case & \#P & Function & v & ఫृ & 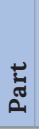 & $\stackrel{\overrightarrow{0}}{\vec{n}}$ & 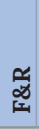 & $z_{i=1}$ & :ே & $\frac{\pi}{\pi}$ & $\sum_{\Sigma}^{5}$ & चี & 覓 & 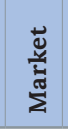 & 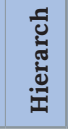 & \\
\hline 15.01 & 1 & $\begin{array}{l}\text { Manager IT Delivery and Support } \\
\text { Consumer Finance }\end{array}$ & $\mathrm{C}$ & $\mathrm{E}$ & $\mathrm{D}$ & $\mathrm{C}$ & $\mathrm{D}$ & B & $\mathrm{C}$ & $\mathrm{E}$ & $\mathrm{E}$ & 26,7 & 21,7 & 23,3 & 28,3 & $28 \%$ \\
\hline 15.01 & 2 & IT Governance Consultant & 0 & 0 & B & A & $\mathrm{C}$ & A & A & 0 & 0 & 43,1 & 24,8 & 13,6 & 18,6 & $56 \%$ \\
\hline 15.01 & 3 & Operational Specialist & 0 & $\mathrm{E}$ & 0 & $\mathrm{C}$ & 0 & $\mathrm{D}$ & $\mathrm{C}$ & A & 0 & 36,7 & 13,3 & 16,7 & 33,3 & $33 \%$ \\
\hline 15.01 & 4 & Business Information Manager & 0 & A & $\mathrm{D}$ & 0 & 0 & $\mathrm{C}$ & B & $\mathrm{C}$ & A & 35,8 & 19,2 & 12,5 & 32,5 & $28 \%$ \\
\hline 15.01 & 5 & Student group & B & $\mathrm{C}$ & $\mathrm{C}$ & $\mathrm{C}$ & $\mathrm{C}$ & $\mathrm{C}$ & B & B & $\mathrm{A}$ & 35,8 & 20,8 & 15,8 & 27,5 & $50 \%$ \\
\hline 15.02 & 1 & Business Analist & $\mathrm{C}$ & B & 0 & 0 & A & $\mathrm{D}$ & $\mathrm{C}$ & $\mathrm{C}$ & A & 30,8 & 12,5 & 15,0 & 41,7 & $50 \%$ \\
\hline 15.02 & 2 & Project manager & B & 0 & B & A & A & $\mathrm{D}$ & $\mathrm{C}$ & 0 & 0 & 42,5 & 11,7 & 4,2 & 41,7 & $61 \%$ \\
\hline 15.02 & 3 & Manager & $\mathrm{A}$ & 0 & $\mathrm{C}$ & B & $\mathrm{C}$ & $\mathrm{A}$ & 0 & A & $\mathrm{A}$ & 33,3 & 7,5 & 19,2 & 40,0 & $50 \%$ \\
\hline 15.02 & 4 & Student group & $\mathrm{B}$ & 0 & B & C & 0 & A & $\mathrm{C}$ & $\mathrm{A}$ & A & 33,3 & 10,0 & 18,3 & 38,3 & $67 \%$ \\
\hline 15.03 & 1 & Manager & $\mathrm{E}$ & C & $\mathrm{A}$ & A & A & A & 0 & 0 & 0 & 6,7 & 20,8 & 40,0 & 32,5 & $39 \%$ \\
\hline 15.03 & 2 & Senior Lean Agile Consultant & A & $\mathrm{D}$ & $\mathrm{D}$ & $\mathrm{C}$ & A & A & B & 0 & 0 & 18,3 & 22,5 & 35,8 & 23,3 & $28 \%$ \\
\hline 15.03 & 3 & Student & $\mathrm{E}$ & C & $\mathrm{A}$ & A & A & $\mathrm{A}$ & 0 & 0 & 0 & 6,7 & 20,8 & 40,0 & 32,5 & $39 \%$ \\
\hline 15.03 & 4 & Student & A & D & D & C & A & A & B & 0 & 0 & 18,3 & 22,5 & 35,8 & 23,3 & $28 \%$ \\
\hline 15.04 & 1 & Site IT Business Analyst & 0 & 0 & B & A & A & A & $\mathrm{A}$ & A & 0 & 23,3 & 19,2 & 23,3 & 34,2 & $67 \%$ \\
\hline 15.04 & 2 & IM/IT Manager Pernis and Moerdijk & $\mathrm{C}$ & E & $\mathrm{A}$ & $\mathrm{C}$ & $\mathrm{E}$ & A & A & B & B & 20,0 & 11,7 & 34,2 & 34,2 & $39 \%$ \\
\hline 15.04 & 3 & Production Specialist & A & $\mathrm{E}$ & A & 0 & $\mathrm{D}$ & 0 & 0 & 0 & 0 & 14,2 & 27,5 & 36,7 & 21,7 & $39 \%$ \\
\hline 15.04 & 4 & Student group & B & 0 & B & $\mathrm{C}$ & 0 & $\mathrm{~A}$ & $\mathrm{C}$ & 0 & 0 & 33,3 & 10,0 & 18,3 & 38,3 & $67 \%$ \\
\hline 15.05 & 1 & Information manager & A & A & A & A & 0 & A & 0 & A & $\mathrm{B}$ & 35,8 & 22,5 & 8,3 & 33,3 & $50 \%$ \\
\hline 15.05 & 2 & Information manager & A & B & B & B & 0 & B & B & $\mathrm{C}$ & A & 31,7 & 18,3 & 19,2 & 30,8 & $44 \%$ \\
\hline 15.05 & 3 & Advisory Corporate IT-Governance & A & 0 & A & 0 & $\mathrm{E}$ & $\mathrm{C}$ & B & $\mathrm{D}$ & $\mathrm{E}$ & 36,7 & 26,7 & 15,0 & 21,7 & $72 \%$ \\
\hline 15.05 & 4 & Division manager & $\mathrm{C}$ & B & B & 0 & B & A & 0 & 0 & B & 36,7 & 14,2 & 14,2 & 35,0 & $50 \%$ \\
\hline 15.05 & 5 & Division manager ICT & $\mathrm{C}$ & $\mathrm{E}$ & $\mathrm{D}$ & $\mathrm{C}$ & $\mathrm{E}$ & $\mathrm{E}$ & $\mathrm{E}$ & $\mathrm{D}$ & $\mathrm{C}$ & 30,8 & 20,0 & 26,7 & 22,5 & $39 \%$ \\
\hline 15.05 & 6 & CSO (Chief Security Officer) & A & C & B & B & B & B & B & $\mathrm{C}$ & $\mathrm{C}$ & 24,2 & 5,8 & 33,3 & 36,7 & $67 \%$ \\
\hline 15.05 & 7 & Chief strategy and external relations & $\mathrm{C}$ & $\mathrm{D}$ & A & B & $\mathrm{C}$ & $\mathrm{C}$ & $\mathrm{C}$ & $\mathrm{C}$ & $\mathrm{C}$ & 38,3 & 35,0 & 13,3 & 13,3 & $39 \%$ \\
\hline
\end{tabular}

Table 77 Results case study 1-5 in 2015 (end result) 


\begin{tabular}{|c|c|c|c|c|c|c|c|c|c|c|c|c|c|c|c|c|}
\hline \multicolumn{3}{|c|}{ Extract from case study database v. 0.82} & \multicolumn{4}{|c|}{$\begin{array}{c}\text { Soft } \\
\text { governance }\end{array}$} & \multicolumn{5}{|c|}{$\begin{array}{c}\text { Hard } \\
\text { governance }\end{array}$} & \multicolumn{4}{|c|}{ Culture } & \multirow[b]{2}{*}{ 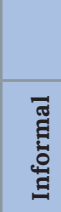 } \\
\hline Case & \#P & Function & च & ఫ్త్త & 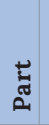 & 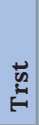 & $\underset{\substack{q \\
\infty}}{\infty}$ & $z$ & ग्ड & $\frac{\mathfrak{\pi}}{\mathbb{a}}$ & $\sum^{0}$ & ีㅠ & 娄 & 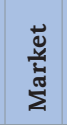 & 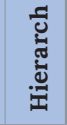 & \\
\hline 15.06 & 1 & HR manager & 0 & A & A & 0 & $\mathrm{C}$ & 0 & 0 & 0 & 0 & 36,7 & 9,2 & 21,7 & 32,5 & $44 \%$ \\
\hline 15.06 & 2 & IT manager & 0 & $\mathrm{~A}$ & B & A & $\mathrm{C}$ & A & 0 & B & 0 & 30,8 & 13,3 & 30,0 & 25,8 & $44 \%$ \\
\hline 15.06 & 3 & Systeem administrator & A & $\mathrm{A}$ & B & B & 0 & 0 & 0 & 0 & 0 & 41,7 & 18,3 & 26,7 & 13,3 & $28 \%$ \\
\hline 15.07 & 1 & IT manager & A & $\mathrm{C}$ & 0 & 0 & $\mathrm{D}$ & A & $\mathrm{C}$ & $\mathrm{C}$ & $\mathrm{D}$ & 6,7 & 9,2 & 37,5 & 46,7 & $0 \%$ \\
\hline 15.07 & 2 & Information manager & 0 & 0 & B & 0 & $\mathrm{~A}$ & A & 0 & $\mathrm{~A}$ & $\mathrm{~A}$ & 23,3 & 23,3 & 40,8 & 12,5 & $50 \%$ \\
\hline 15.07 & 3 & SAP consultant & 0 & 0 & 0 & 0 & $\mathrm{~A}$ & A & $\mathrm{A}$ & $\mathrm{C}$ & $\mathrm{C}$ & 36,7 & 22,5 & 13,3 & 27,5 & $50 \%$ \\
\hline 15.08 & 1 & Purchasing manager & 0 & $\mathrm{~B}$ & B & $\mathrm{A}$ & $\mathrm{A}$ & 0 & $\mathrm{~A}$ & A & 0 & 33,3 & 15,8 & 12,5 & 38,3 & $72 \%$ \\
\hline 15.08 & 2 & Programma manager & A & A & B & A & $\mathrm{A}$ & A & $\mathrm{A}$ & 0 & 0 & 27,5 & 25,8 & 20,8 & 25,8 & $67 \%$ \\
\hline 15.08 & 3 & Enterprise architect & 0 & 0 & B & $\mathrm{A}$ & $\mathrm{A}$ & A & $\mathrm{A}$ & 0 & 0 & 33,3 & 18,3 & 16,7 & 31,7 & $50 \%$ \\
\hline 15.08 & 4 & Servicelevel manager & 0 & B & B & A & $\mathrm{A}$ & A & $\mathrm{A}$ & 0 & 0 & 33,3 & 20,0 & 20,0 & 26,7 & $56 \%$ \\
\hline 15.08 & 5 & Service manager & B & A & B & B & $\mathrm{A}$ & A & $\mathrm{A}$ & 0 & 0 & 28,3 & 14,2 & 14,2 & 43,3 & $50 \%$ \\
\hline 15.08 & 6 & Manager Informatie \& Automatisering & A & B & B & B & B & B & A & A & 0 & 39,2 & 15,8 & 16,7 & 28,3 & $61 \%$ \\
\hline 15.09 & 1 & Business line manager & 0 & A & B & 0 & 0 & $\mathrm{C}$ & 0 & 0 & 0 & 23,3 & 27,5 & 27,5 & 21,7 & $61 \%$ \\
\hline 15.09 & 2 & Innovation manager (strategic level) & A & A & $\mathrm{C}$ & A & 0 & $\mathrm{C}$ & $\mathrm{A}$ & B & B & 33,3 & 15,0 & 28,3 & 23,3 & $67 \%$ \\
\hline 15.09 & 3 & Manager system development & 0 & 0 & $\mathrm{C}$ & 0 & 0 & 0 & $\mathrm{~A}$ & 0 & 0 & 20,3 & 19,8 & 45,3 & 14,5 & $56 \%$ \\
\hline 15.10 & 1 & Corporate ICT manager & 0 & 0 & B & $\mathrm{C}$ & D & C & A & $\mathrm{C}$ & $\mathrm{C}$ & 10 & 11,7 & 51,7 & 26,7 & $11 \%$ \\
\hline 15.10 & 2 & Product Developer & 0 & A & B & 0 & $\mathrm{~A}$ & A & B & 0 & 0 & 16,7 & 20,0 & 38,3 & 25,0 & $50 \%$ \\
\hline \multirow[t]{2}{*}{15.10} & 3 & SAP Application Consultant & A & A & 0 & $\mathrm{C}$ & $\mathrm{A}$ & B & 0 & A & 0 & 15,0 & 13,3 & 45,8 & 25,8 & $44 \%$ \\
\hline & 42 & \multicolumn{15}{|l|}{ Participants } \\
\hline \multirow[t]{2}{*}{2015} & 10 & Cases & & & & & & & Min.: & & & 6,7 & 9,2 & 12,5 & 12,5 & $0 \%$ \\
\hline & 42 & Participants & & & & & & & Max.: & & & 41,7 & 27,5 & 51,7 & 46,7 & $72 \%$ \\
\hline
\end{tabular}

Table 78 Results case study 6-10 in 2015 (end result) 


\section{F.2 Results case studies in 2016}

\begin{tabular}{|c|c|c|c|c|c|c|c|c|c|c|c|}
\hline \multicolumn{3}{|c|}{ Extract from case study database v. 0.82} & \multicolumn{4}{|c|}{ Hard governance } & \multicolumn{5}{|c|}{ Soft governance } \\
\hline Case & \#P & Function & CI & Lead & Part & Trst & F\&R & FN & Deci & Plan & Mon \\
\hline 16.01 & 1 & ICT manager & $\mathrm{A}$ & A & $\mathrm{A}$ & $\mathrm{A}$ & A & A & $\mathrm{C}$ & $\mathrm{D}$ & B \\
\hline 16.01 & 2 & $\mathrm{CIO}$ & $\mathrm{E}$ & $\mathrm{F}$ & A & $\mathrm{D}$ & $\mathrm{E}$ & A & $\mathrm{C}$ & $\mathrm{C}$ & $\mathrm{E}$ \\
\hline 16.01 & 3 & Manager ITO & $\mathrm{A}$ & A & $\mathrm{A}$ & $\mathrm{A}$ & A & A & A & $\mathrm{D}$ & $\mathrm{A}$ \\
\hline 16.02 & 1 & IT Director & $\mathrm{A}$ & A & A & A & E & A & A & B & $\mathrm{A}$ \\
\hline 16.02 & 2 & IT Governance Consultant & A & $\mathrm{D}$ & B & A & A & A & A & A & A \\
\hline 16.02 & 3 & Manager IT Infrastructure Europe & A & A & A & A & A & A & $\mathrm{C}$ & A & A \\
\hline 16.02 & 4 & Student group & $\mathrm{A}$ & A & B & $\mathrm{A}$ & B & A & A & $\mathrm{A}$ & $\mathrm{A}$ \\
\hline 16.03 & 1 & IT architect & $\mathrm{A}$ & B & B & B & A & $\mathrm{C}$ & $\mathrm{C}$ & $\mathrm{C}$ & A \\
\hline 16.03 & 2 & Lean navigator & A & A & $\mathrm{C}$ & B & $\mathrm{C}$ & A & A & A & A \\
\hline 16.03 & 3 & Department manager delivery & A & A & B & A & A & $\mathrm{D}$ & A & A & A \\
\hline 16.03 & 4 & Student group & $\mathrm{A}$ & $\mathrm{B}$ & A & A & $\mathrm{C}$ & $\mathrm{C}$ & $\mathrm{C}$ & $\mathrm{D}$ & $\mathrm{C}$ \\
\hline 16.04 & 1 & Chairman of the Board & A & B & $\mathrm{C}$ & A & A & $\mathrm{C}$ & A & $\mathrm{E}$ & $\mathrm{D}$ \\
\hline 16.04 & 2 & Director Finance and Control & A & A & A & A & A & $\mathrm{C}$ & $\mathrm{C}$ & $\mathrm{D}$ & $\mathrm{C}$ \\
\hline 16.04 & 3 & ICT Manager & A & B & B & A & A & A & A & A & A \\
\hline 16.04 & 4 & Director business line & A & A & A & B & A & A & A & A & B \\
\hline 16.04 & 5 & Manager serviceline and helpdesk & A & B & A & A & A & A & A & A & A \\
\hline 16.04 & 6 & Director business line & A & $\mathrm{C}$ & B & A & $\mathrm{D}$ & $\mathrm{C}$ & A & $\mathrm{E}$ & $\mathrm{C}$ \\
\hline 16.04 & 7 & Enterprise manager ICT & $\mathrm{C}$ & B & A & A & A & $\mathrm{E}$ & $\mathrm{E}$ & $\mathrm{C}$ & $\mathrm{E}$ \\
\hline 16.05 & 1 & Lead Architect & $\mathrm{E}$ & B & $\mathrm{C}$ & B & A & A & B & A & A \\
\hline 16.05 & 2 & $\begin{array}{l}\text { Head of business architecture and } \\
\text { design }\end{array}$ & A & B & A & A & A & A & $\mathrm{C}$ & $\mathrm{E}$ & A \\
\hline 16.05 & 3 & Student group & $\mathrm{D}$ & B & $\mathrm{C}$ & B & $\mathrm{C}$ & B & $\mathrm{C}$ & $\mathrm{E}$ & $\mathrm{C}$ \\
\hline 16.06 & 1 & $\begin{array}{l}\text { CIO of Thales Nederland and head } \\
\text { of the ISD }\end{array}$ & $\mathrm{E}$ & $\mathrm{C}$ & B & $\mathrm{C}$ & A & A & $\mathrm{C}$ & $\mathrm{E}$ & $\mathrm{C}$ \\
\hline 16.06 & 2 & Manager Applications \& Projects & $\mathrm{E}$ & $\mathrm{D}$ & A & A & $\mathrm{C}$ & $\mathrm{C}$ & $\mathrm{C}$ & $\mathrm{C}$ & $\mathrm{C}$ \\
\hline 16.06 & 3 & $\begin{array}{l}\text { Team leader End User Support \& } \\
\text { Process Owner Incidents }\end{array}$ & A & $\mathrm{F}$ & A & A & $\mathrm{C}$ & $\mathrm{C}$ & $\mathrm{A}$ & B & $\mathrm{C}$ \\
\hline 16.06 & 4 & Quality \& Business Improvement & E & $\mathrm{B}$ & A & A & $\mathrm{C}$ & $\mathrm{C}$ & $\mathrm{C}$ & $\mathrm{C}$ & $\mathrm{C}$ \\
\hline 16.07 & 1 & Director & E & $\mathrm{F}$ & B & $\mathrm{C}$ & E & A & B & B & B \\
\hline 16.07 & 2 & Head of IT Projects \& Development & A & B & A & A & A & A & A & A & A \\
\hline 16.07 & 3 & $\begin{array}{l}\text { Head demand and supply } \\
\text { management }\end{array}$ & E & $\mathrm{E}$ & A & $\mathrm{D}$ & A & A & B & $\mathrm{A}$ & A \\
\hline 16.07 & 4 & IT accout and product manager & A & A & B & $\mathrm{C}$ & $\mathrm{E}$ & $\mathrm{E}$ & $\mathrm{C}$ & E & $\mathrm{D}$ \\
\hline
\end{tabular}




\begin{tabular}{|c|c|c|c|c|c|c|c|c|c|c|c|}
\hline \multicolumn{3}{|c|}{ Extract from case study database v. 0.82} & \multicolumn{4}{|c|}{ Hard governance } & \multicolumn{5}{|c|}{ Soft governance } \\
\hline Case & \#P & Function & CI & Lead & Part & Trst & F\&R & FN & Deci & Plan & Mon \\
\hline 16.07 & 5 & $\begin{array}{l}\text { Head of University Business } \\
\text { Information Management }\end{array}$ & A & B & A & B & A & B & $\mathrm{C}$ & $\mathrm{C}$ & $\mathrm{C}$ \\
\hline 16.08 & 1 & $\begin{array}{l}\text { Information Manager CIO Office } \\
\text { (and dept CIO) }\end{array}$ & A & $\mathrm{A}$ & $\mathrm{C}$ & A & $\mathrm{A}$ & $\mathrm{C}$ & $\mathrm{C}$ & $\mathrm{A}$ & B \\
\hline 16.08 & 2 & Unitmanager IT Development & B & A & A & $\mathrm{C}$ & $\mathrm{C}$ & A & A & A & A \\
\hline 16.08 & 3 & $\begin{array}{l}\text { Director of Information } \\
\text { Management \& Consulting, } \\
\text { Informatiemanager }\end{array}$ & A & A & B & B & $\mathrm{D}$ & A & B & A & A \\
\hline & 33 & \multicolumn{10}{|l|}{ Participants } \\
\hline \multirow[t]{2}{*}{2016} & 8 & Cases & & & & & & & & & \\
\hline & 33 & Participants & & & & & & & & & \\
\hline
\end{tabular}

Table 79 Results case study 1-10 in 2016, hard and soft governance (end result)

\begin{tabular}{|c|c|c|c|c|c|c|c|c|c|c|c|c|c|c|c|c|c|}
\hline \multicolumn{2}{|c|}{ Extract } & \multicolumn{4}{|c|}{ Culture } & \multicolumn{9}{|c|}{ Informal organization } & \multirow{2}{*}{\multicolumn{3}{|c|}{ Soft HardInformal }} \\
\hline Case & \#P & Clan & Adh & Market & Hierarch & CI & Lead & Part & Trst & $F \& R$ & FN & Deci & Plan & Mon & & & \\
\hline 16.01 & 1 & 48,3 & 10,8 & 13,3 & 27,5 & 20 & 40 & 50 & 70 & 30 & 30 & 20 & 80 & 30 & $45 \%$ & $38 \%$ & $41 \%$ \\
\hline 16.01 & 2 & 38,3 & 19,2 & 18,3 & 24,2 & 70 & 70 & 50 & 50 & 50 & 40 & 40 & 50 & 30 & $60 \%$ & $42 \%$ & $50 \%$ \\
\hline 16.01 & 3 & 48,3 & 12,5 & 15,0 & 24,2 & 40 & 40 & 60 & 60 & 50 & 50 & 70 & 80 & 50 & $50 \%$ & $60 \%$ & $56 \%$ \\
\hline 16.02 & 1 & 20,0 & 26,7 & 41,7 & 11,7 & 50 & 30 & 80 & 50 & 30 & 20 & 30 & 30 & 50 & $53 \%$ & $32 \%$ & $41 \%$ \\
\hline 16.02 & 2 & 59,2 & 20,8 & 11,7 & 8,3 & 30 & 40 & 40 & 60 & 20 & 30 & 10 & 40 & 40 & $43 \%$ & $28 \%$ & $34 \%$ \\
\hline 16.02 & 3 & 32,5 & 20,8 & 23,3 & 23,3 & 30 & 50 & 70 & 80 & 40 & 40 & 20 & 70 & 40 & $58 \%$ & $42 \%$ & $49 \%$ \\
\hline 16.02 & 4 & 40,0 & 20,8 & 23,3 & 15,8 & 30 & 40 & 50 & 60 & 30 & 30 & 40 & 35 & 40 & $45 \%$ & $35 \%$ & $39 \%$ \\
\hline 16.03 & 1 & 26,3 & 12,7 & 19,3 & 41,7 & 30 & 20 & 60 & 40 & 10 & 10 & 10 & 20 & 20 & $38 \%$ & $14 \%$ & $24 \%$ \\
\hline 16.03 & 2 & 14,2 & 6,7 & 29,2 & 50,0 & 50 & 50 & 30 & 50 & 25 & 30 & 40 & 50 & 30 & $45 \%$ & $35 \%$ & $39 \%$ \\
\hline 16.03 & 3 & 15,0 & 6,7 & 21,7 & 56,7 & 20 & 20 & 40 & 30 & 20 & 20 & 40 & 40 & 20 & $28 \%$ & $28 \%$ & $28 \%$ \\
\hline 16.03 & 4 & 14,2 & 5,0 & 31,7 & 49,2 & 25 & 40 & 50 & 35 & 30 & 20 & 20 & 30 & 35 & $38 \%$ & $27 \%$ & $32 \%$ \\
\hline 16.04 & 1 & 61,7 & 15,0 & 7,5 & 15,8 & 20 & 30 & 50 & 30 & 60 & 50 & 50 & 20 & 60 & $33 \%$ & $48 \%$ & $41 \%$ \\
\hline 16.04 & 2 & 39,0 & 13,0 & 24,0 & 24,0 & 40 & 50 & 60 & 50 & 30 & 30 & 20 & 20 & 30 & $50 \%$ & $26 \%$ & $37 \%$ \\
\hline 16.04 & 3 & 36,7 & 21,7 & 17,5 & 24,2 & 20 & 10 & 60 & 30 & 20 & 10 & 50 & 50 & 70 & $30 \%$ & $40 \%$ & $36 \%$ \\
\hline 16.04 & 4 & 25,0 & 13,8 & 21,3 & 40,0 & 10 & 20 & 40 & 30 & 25 & 10 & 20 & 70 & 25 & $25 \%$ & $30 \%$ & $28 \%$ \\
\hline 16.04 & 5 & 28,3 & 25,0 & 17,5 & 29,2 & 30 & 10 & 70 & 10 & 30 & 20 & 40 & 40 & 80 & $30 \%$ & $42 \%$ & $37 \%$ \\
\hline 16.04 & 6 & 23,3 & 8,3 & 20,0 & 48,3 & 40 & 70 & 40 & 70 & 20 & 20 & 60 & 40 & 50 & $55 \%$ & $38 \%$ & $46 \%$ \\
\hline 16.04 & 7 & 26,7 & 35,0 & 26,7 & 11,7 & 60 & 30 & 40 & 30 & 60 & 30 & 30 & 30 & 20 & $40 \%$ & $34 \%$ & $37 \%$ \\
\hline 16.05 & 1 & 33,3 & 9,2 & 12,5 & 45,0 & 20 & 10 & 20 & 30 & 20 & 20 & 10 & 20 & 0 & $20 \%$ & $14 \%$ & $17 \%$ \\
\hline
\end{tabular}




\section{Appendix F}

\begin{tabular}{|c|c|c|c|c|c|c|c|c|c|c|c|c|c|c|c|c|c|}
\hline \multicolumn{2}{|c|}{ Extract } & \multicolumn{4}{|c|}{ Culture } & \multicolumn{9}{|c|}{ Informal organization } & \multirow[b]{2}{*}{ Soft } & \multirow[b]{2}{*}{ HardI } & \multirow[b]{2}{*}{ Informal } \\
\hline Case & \#P & Clan & Adh & Market & Hierarch & CI & Lead & Part & Trst & $F \& R$ & FN & Deci & i Plan & Mon & & & \\
\hline 16.05 & 2 & 20,0 & 20,0 & 25,0 & 35,0 & 25 & 40 & 50 & 35 & 30 & 20 & 20 & 30 & 35 & $38 \%$ & $27 \%$ & $32 \%$ \\
\hline 16.05 & 3 & 19,2 & 8,3 & 30,8 & 41,7 & n.b & 30 & 20 & 40 & 20 & 30 & 10 & 25 & 50 & $30 \%$ & $27 \%$ & $28 \%$ \\
\hline 16.06 & 1 & 43,3 & 36,7 & 5,0 & 15,0 & 50 & 30 & 70 & 70 & 50 & 50 & 30 & 30 & 30 & $55 \%$ & $38 \%$ & $46 \%$ \\
\hline 16.06 & 2 & 33,3 & 18,3 & 20,0 & 28,3 & 50 & 20 & 30 & 60 & 40 & 30 & 30 & 30 & 40 & $40 \%$ & $34 \%$ & $37 \%$ \\
\hline 16.06 & 3 & 38,3 & 16,7 & 16,7 & 28,3 & 80 & 40 & 50 & 20 & 20 & 20 & 30 & 40 & 40 & $48 \%$ & $30 \%$ & $38 \%$ \\
\hline 16.06 & 4 & 29,2 & 14,6 & 22,9 & 33,3 & 30 & 70 & 70 & 80 & 80 & 50 & 50 & 80 & 0 & $63 \%$ & $52 \%$ & $57 \%$ \\
\hline 16.07 & 1 & 40,8 & 25,0 & 7,5 & 26,7 & 50 & 20 & 60 & 30 & 10 & 70 & 30 & 30 & 40 & $40 \%$ & $36 \%$ & $38 \%$ \\
\hline 16.07 & 2 & 30,0 & 20,8 & 20,8 & 28,3 & n.a. & n.a. & 60 & 30 & 20 & 30 & 20 & 20 & 20 & $45 \%$ & $22 \%$ & $29 \%$ \\
\hline 16.07 & 3 & 31,8 & 21,8 & 26,7 & 19,7 & 20 & 30 & 80 & 40 & 60 & 50 & 20 & 30 & 30 & $43 \%$ & $38 \%$ & $40 \%$ \\
\hline 16.07 & 4 & 27,5 & 28,3 & 20,0 & 24,2 & 50 & 50 & 70 & 40 & 20 & 60 & 40 & 30 & 30 & $53 \%$ & $36 \%$ & $43 \%$ \\
\hline 16.07 & 5 & 26,7 & 24,2 & 18,3 & 30,8 & 50 & 30 & 50 & 30 & 20 & 50 & 20 & 50 & 20 & $40 \%$ & $32 \%$ & $36 \%$ \\
\hline 16.08 & 1 & 27,5 & 20,0 & 20,8 & 31,7 & 20 & 40 & 70 & 60 & 20 & 60 & 70 & 80 & 80 & $48 \%$ & $62 \%$ & $56 \%$ \\
\hline 16.08 & 2 & 36,7 & 12,5 & 11,7 & 39,2 & 60 & 50 & 50 & 50 & 20 & 20 & 30 & 50 & 50 & $53 \%$ & $34 \%$ & $42 \%$ \\
\hline 16.08 & 3 & 29,2 & 20,8 & 22,5 & 27,5 & 75 & 60 & 50 & 65 & 55 & 65 & 35 & 30 & 40 & $63 \%$ & $45 \%$ & $53 \%$ \\
\hline \multicolumn{6}{|c|}{33} & & & & & & & & & & & & \\
\hline \multirow[t]{2}{*}{2016} & Min: & 14,2 & 5,0 & 5,0 & 8,3 & 10 & 10 & 20 & 10 & 10 & 10 & 10 & 20 & 0 & $20 \%$ & $14 \%$ & $17 \%$ \\
\hline & Max: & 61,7 & 36,7 & 41,7 & 56,7 & 80 & 70 & 80 & 80 & 80 & 70 & 70 & 80 & 80 & $63 \%$ & $62 \%$ & $57 \%$ \\
\hline
\end{tabular}

Table 80 Results case study 1-10 in 2016, context (end result) 


\section{F.3 Results case studies in 2017}

\begin{tabular}{|c|c|c|c|c|c|c|c|c|c|c|c|}
\hline \multicolumn{3}{|c|}{ Extract from case study database v. 0.82} & \multicolumn{9}{|c|}{ View: Department } \\
\hline Case & \#P & Function & CI & Lead & Part & Trst & F\&R & FN & Deci & Plan & Mon \\
\hline 17.01 & 1 & Service Process Consultant & $\mathrm{E}$ & $\mathrm{D}$ & $\mathrm{D}$ & $\mathrm{A}$ & $\mathrm{E}$ & A & $\mathrm{A}$ & A & $\mathrm{A}$ \\
\hline 17.01 & 2 & Program Manager Lean IT transformation & B & $\mathrm{F}$ & $\mathrm{A}$ & $\mathrm{B}$ & $\mathrm{A}$ & A & $\mathrm{A}$ & A & $\mathrm{E}$ \\
\hline 17.01 & 3 & Teamlead IT & A & B & A & $\mathrm{A}$ & $\mathrm{A}$ & A & $\mathrm{C}$ & $\mathrm{A}$ & A \\
\hline 17.01 & 4 & Manager & $\mathrm{D}$ & $\mathrm{E}$ & A & A & $\mathrm{D}$ & A & $\mathrm{D}$ & $\mathrm{A}$ & B \\
\hline 17.02 & 1 & n.b. & $\mathrm{A}$ & B & A & B & $\mathrm{A}$ & B & $\mathrm{C}$ & $\mathrm{C}$ & $\mathrm{C}$ \\
\hline 17.02 & 2 & n.b. & A & A & A & $\mathrm{A}$ & $\mathrm{A}$ & $\mathrm{C}$ & A & A & $\mathrm{C}$ \\
\hline 17.02 & 3 & n.b. & A & E & A & A & $\mathrm{A}$ & B & A & A & A \\
\hline 17.02 & 4 & n.b. & $\mathrm{A}$ & E & A & $\mathrm{A}$ & $\mathrm{A}$ & A & $\mathrm{E}$ & $\mathrm{C}$ & A \\
\hline 17.02 & 5 & n.b. & A & B & A & A & A & $\mathrm{A}$ & A & A & A \\
\hline 17.03 & 1 & Global Head Lending \& Trade & A & B & A & A & $\mathrm{C}$ & B & $\mathrm{A}$ & A & A \\
\hline 17.03 & 2 & IT manager & $\mathrm{A}$ & B & A & $\mathrm{A}$ & $\mathrm{A}$ & A & A & A & A \\
\hline 17.03 & 3 & Manager & $\mathrm{D}$ & E & A & $\mathrm{D}$ & $\mathrm{A}$ & A & $\mathrm{A}$ & $\mathrm{A}$ & $\mathrm{C}$ \\
\hline 17.03 & 4 & Student group & $\mathrm{CI}$ & $\mathrm{F}$ & B & B & B & $\mathrm{E}$ & $\mathrm{C}$ & B & B \\
\hline 17.04 & 1 & E-commerce Business Process Manager & A & A & A & $\mathrm{C}$ & A & A & A & A & A \\
\hline 17.04 & 2 & iOS Developer & B & $\mathrm{F}$ & $\mathrm{D}$ & A & $\mathrm{E}$ & A & $\mathrm{E}$ & $\mathrm{E}$ & B \\
\hline 17.04 & 3 & Coorinator & A & F & A & $\mathrm{D}$ & A & A & E & B & A \\
\hline 17.04 & 4 & iOS Developer & $\mathrm{D}$ & $\mathrm{F}$ & A & $\mathrm{C}$ & $\mathrm{E}$ & A & $\mathrm{E}$ & A & E \\
\hline 17.04 & 5 & Assistent Engineer & $\mathrm{E}$ & $\mathrm{F}$ & A & $\mathrm{C}$ & A & $\mathrm{C}$ & A & $\mathrm{E}$ & A \\
\hline 17.04 & 6 & Backend Developer & A & $\mathrm{F}$ & A & A & A & A & A & E & A \\
\hline 17.04 & 7 & Team lead/scrum master & A & $\mathrm{A}$ & A & $\mathrm{A}$ & A & A & $\mathrm{A}$ & A & $\mathrm{A}$ \\
\hline 17.04 & 8 & Mobile Engineer & $\mathrm{D}$ & $\mathrm{D}$ & A & A & A & $\mathrm{A}$ & $\mathrm{D}$ & A & A \\
\hline 17.05 & 1 & Manager TCE-NL & A & A & A & A & A & A & A & A & A \\
\hline 17.05 & 2 & Manager sw engineering team & $\mathrm{E}$ & B & $\mathrm{D}$ & A & $\mathrm{E}$ & A & $\mathrm{E}$ & E & E \\
\hline 17.05 & 3 & IS/IT Manager & $\mathrm{D}$ & $\mathrm{F}$ & A & $\mathrm{D}$ & $\mathrm{A}$ & A & E & E & A \\
\hline 17.05 & 4 & IS/IT Solution Engineer & A & $\mathrm{F}$ & A & A & A & B & $\mathrm{C}$ & B & A \\
\hline 17.05 & 5 & Spares \& Repairs Manager & A & $\mathrm{A}$ & $\mathrm{A}$ & A & A & $\mathrm{A}$ & A & A & $\mathrm{A}$ \\
\hline 17.06 & 1 & Warehouse Supervisor & A & A & A & A & A & A & $\mathrm{A}$ & A & $\mathrm{A}$ \\
\hline 17.06 & 2 & E-shop manager & A & A & A & A & A & A & A & A & A \\
\hline 17.06 & 3 & Order picker & $\mathrm{A}$ & A & A & A & A & A & A & A & A \\
\hline 17.06 & 4 & Production employee ICT department & A & $\mathrm{F}$ & A & $\mathrm{C}$ & A & A & A & A & A \\
\hline 17.06 & 5 & Order picker & A & B & $\mathrm{A}$ & $\mathrm{A}$ & B & $\mathrm{A}$ & $\mathrm{A}$ & $\mathrm{A}$ & $\mathrm{A}$ \\
\hline
\end{tabular}




\begin{tabular}{|c|c|c|c|c|c|c|c|c|c|c|c|}
\hline \multicolumn{3}{|c|}{ Extract from case study database v. 0.82} & \multicolumn{9}{|c|}{ View: Department } \\
\hline Case & \#P & Function & CI & Lead & Part & Trst & F\&R & FN & Deci & Plan & Mon \\
\hline 17.07 & 1 & Consultant & A & A & $\mathrm{A}$ & A & A & A & A & $\mathrm{A}$ & A \\
\hline 17.07 & 2 & Business Unit Manager & $\mathrm{E}$ & B & A & A & A & $\mathrm{E}$ & $\mathrm{E}$ & $\mathrm{E}$ & $\mathrm{E}$ \\
\hline 17.07 & 3 & Consultant & A & $\mathrm{D}$ & A & A & A & A & A & A & A \\
\hline 17.07 & 4 & Consultant & E & $\mathrm{F}$ & A & B & E & A & A & E & A \\
\hline 17.07 & 5 & Business Manager & B & $\mathrm{F}$ & A & $\mathrm{A}$ & $\mathrm{E}$ & $\mathrm{E}$ & $\mathrm{E}$ & $\mathrm{E}$ & $\mathrm{E}$ \\
\hline 17.08 & 1 & Team coordinator SAP & A & $\mathrm{F}$ & $\mathrm{A}$ & A & A & A & A & A & $\mathrm{E}$ \\
\hline 17.08 & 2 & Coordinator technical application design & A & $\mathrm{E}$ & A & $\mathrm{D}$ & B & A & $\mathrm{E}$ & $\mathrm{E}$ & $\mathrm{E}$ \\
\hline 17.08 & 3 & Technical coordinator & A & $\mathrm{E}$ & A & A & $\mathrm{C}$ & A & A & A & E \\
\hline 17.08 & 4 & Enterprise Architect & A & A & A & A & A & A & A & $\mathrm{A}$ & A \\
\hline 17.08 & 5 & Teamcoordinator ICT beheer \& helpdesk & A & E & A & A & A & A & A & A & A \\
\hline 17.08 & 6 & ICT manager (CIO) & A & $\mathrm{F}$ & B & A & A & B & $\mathrm{E}$ & A & E \\
\hline 17.08 & 7 & Projectleider ICS & A & $\mathrm{C}$ & A & A & A & A & A & A & A \\
\hline 17.09 & 1 & Coordinator Enterprise Architecture & A & B & B & B & B & B & A & B & B \\
\hline 17.09 & 2 & Senior adviseur Informatisering & A & $\mathrm{C}$ & B & $\mathrm{C}$ & $\mathrm{D}$ & $\mathrm{D}$ & $\mathrm{C}$ & B & B \\
\hline 17.09 & 3 & Head Risc manager & $\mathrm{C}$ & $\mathrm{D}$ & $\mathrm{D}$ & $\mathrm{D}$ & $\mathrm{C}$ & $\mathrm{C}$ & $\mathrm{C}$ & $\mathrm{C}$ & $\mathrm{C}$ \\
\hline 17.09 & 4 & Senior projectleader & A & $\mathrm{D}$ & A & B & B & A & A & B & A \\
\hline 17.09 & 5 & Manager program management department & A & E & B & $\mathrm{C}$ & B & B & B & A & $\mathrm{C}$ \\
\hline 17.09 & 6 & Department manager & A & $\mathrm{F}$ & A & $\mathrm{C}$ & $\mathrm{D}$ & $\mathrm{C}$ & A & $\mathrm{C}$ & $\mathrm{C}$ \\
\hline 17.09 & 7 & Coördinerend beleidsmedewerker & A & $\mathrm{D}$ & A & B & B & $\mathrm{C}$ & $\mathrm{B}$ & B & $\mathrm{A}$ \\
\hline 17.10 & 1 & Strategisch adviseur & A & $\mathrm{C}$ & A & A & $\mathrm{C}$ & $\mathrm{D}$ & A & E & B \\
\hline 17.10 & 2 & Project manager & A & A & B & A & A & A & A & A & A \\
\hline 17.10 & 3 & Manager Service Delivery & A & $\mathrm{C}$ & $\mathrm{C}$ & B & $\mathrm{C}$ & $\mathrm{C}$ & $\mathrm{C}$ & $\mathrm{C}$ & $\mathrm{C}$ \\
\hline 17.10 & 4 & Functional designer & A & $\mathrm{D}$ & B & B & $\mathrm{C}$ & A & A & $\mathrm{C}$ & $\mathrm{C}$ \\
\hline 17.10 & 5 & Klant Domein Manager & A & B & $\mathrm{C}$ & A & $\mathrm{C}$ & A & A & A & A \\
\hline 17.10 & 6 & Manager & $\mathrm{C}$ & $\mathrm{D}$ & B & A & $\mathrm{C}$ & A & A & A & B \\
\hline 17.10 & 7 & Vice Director information management & A & A & B & A & A & A & A & A & A \\
\hline 17.10 & 8 & Lead Architect OB & A & B & A & B & B & $\mathrm{C}$ & A & B & B \\
\hline 17.10 & 9 & Project Manager \& Product Manager & B & B & $\mathrm{C}$ & A & A & $\mathrm{C}$ & B & $\mathrm{C}$ & $\mathrm{C}$ \\
\hline \multirow[t]{2}{*}{17.10} & 10 & Projectmanager - Release train engineer & B & B & B & B & A & A & A & B & E \\
\hline & 60 & Participants & & & & & & & & & \\
\hline \multirow[t]{2}{*}{2017} & 10 & Cases & & & & & & & & & \\
\hline & 60 & Participants & & & & & & & & & \\
\hline
\end{tabular}

Table 81 Results case studies 2017, hard and soft governance (department view, end result) 


\begin{tabular}{|c|c|c|c|c|c|c|c|c|c|c|c|c|c|c|c|c|c|}
\hline \multicolumn{2}{|c|}{ Extract } & \multicolumn{4}{|c|}{ Culture } & \multicolumn{9}{|c|}{ Informal organization } & \multirow[b]{2}{*}{ Soft } & \multirow{2}{*}{\multicolumn{2}{|c|}{ Hard Informal }} \\
\hline Case & \#P & Clan & Adh & Market & Hierarch & CI & Lead & Part & Trst & F\&R & FN & Deci & Plan & Mon & & & \\
\hline 17.01 & 1 & 15,8 & 29,2 & 23,3 & 31,7 & 70 & 40 & 50 & 40 & 30 & 30 & 20 & 30 & 40 & $50 \%$ & $30 \%$ & $39 \%$ \\
\hline 17.01 & 2 & 35,0 & 26,7 & 18,3 & 20,0 & 80 & 60 & 70 & 70 & 30 & 40 & 60 & 50 & 60 & $70 \%$ & $48 \%$ & $58 \%$ \\
\hline 17.01 & 3 & 33,9 & 18,6 & 23,7 & 23,7 & 40 & 30 & 70 & 30 & 20 & 20 & 10 & 40 & 70 & $43 \%$ & $32 \%$ & $37 \%$ \\
\hline 17.01 & 4 & 25,0 & 20,0 & 26,7 & 28,3 & 50 & 30 & 80 & 50 & 10 & 10 & 20 & 50 & 20 & $53 \%$ & $22 \%$ & $36 \%$ \\
\hline 17.02 & 1 & 26,7 & 24,2 & 18,3 & 30,8 & 50 & 30 & 50 & 30 & 20 & 50 & 20 & 50 & 20 & $40 \%$ & $32 \%$ & $36 \%$ \\
\hline 17.02 & 2 & 20,0 & 3,3 & 26,7 & 50,0 & 50 & 10 & 70 & 50 & 10 & 60 & 10 & 30 & 20 & $45 \%$ & $26 \%$ & $34 \%$ \\
\hline 17.02 & 3 & 44,2 & 8,3 & 17,5 & 30,0 & 70 & 60 & 80 & 65 & 30 & 50 & 75 & 60 & 70 & $69 \%$ & $57 \%$ & $62 \%$ \\
\hline 17.02 & 4 & 37,5 & 15,0 & 15,0 & 32,5 & n.b. & 40 & 30 & 50 & 30 & 30 & 20 & 40 & 30 & $40 \%$ & $30 \%$ & $34 \%$ \\
\hline 17.02 & 5 & 36,7 & 25,0 & 11,7 & 26,7 & 40 & 40 & 60 & 50 & 50 & 60 & 50 & 60 & 60 & $48 \%$ & $56 \%$ & $52 \%$ \\
\hline 17.03 & 1 & 30,0 & 18,3 & 23,3 & 28,3 & 40 & 30 & 80 & 50 & 30 & 30 & 20 & 60 & 50 & $50 \%$ & $38 \%$ & $43 \%$ \\
\hline 17.03 & 2 & 33,3 & 21,7 & 23,3 & 21,7 & 50 & 30 & 60 & 60 & 30 & 50 & 30 & 50 & 30 & $50 \%$ & $38 \%$ & $43 \%$ \\
\hline 17.03 & 3 & 40,0 & 25,0 & 21,7 & 13,3 & 10 & 20 & 90 & 70 & 50 & 50 & 75 & 10 & 90 & $48 \%$ & $55 \%$ & $52 \%$ \\
\hline 17.03 & 4 & 30,0 & 22,5 & 24,2 & 23,3 & 40 & 30 & 80 & 30 & 60 & 40 & 70 & 60 & 70 & $45 \%$ & $60 \%$ & $53 \%$ \\
\hline 17.04 & 1 & 25,0 & 20,0 & 23,3 & 31,7 & 20 & 50 & 70 & 80 & 70 & 70 & 70 & 50 & 50 & $55 \%$ & $62 \%$ & $59 \%$ \\
\hline 17.04 & 2 & 44,2 & 23,3 & 10,8 & 21,7 & 60 & 50 & 40 & 50 & 30 & 50 & 25 & 10 & 30 & $50 \%$ & $29 \%$ & $38 \%$ \\
\hline 17.04 & 3 & 65,8 & 27,5 & 4,2 & 2,5 & 80 & 10 & 50 & 50 & 100 & 100 & 50 & 100 & 100 & $48 \%$ & $90 \%$ & $71 \%$ \\
\hline 17.04 & 4 & 32,5 & 27,5 & 15,8 & 24,2 & 50 & 40 & 70 & 40 & 60 & 40 & 30 & 30 & 70 & $50 \%$ & $46 \%$ & $48 \%$ \\
\hline 17.04 & 5 & 44,2 & 35,0 & 13,3 & 7,5 & 60 & 40 & 70 & 60 & 70 & 70 & 40 & 40 & 40 & $58 \%$ & $52 \%$ & $54 \%$ \\
\hline 17.04 & 6 & 23,3 & 24,2 & 30,8 & 21,7 & 80 & 100 & 100 & 90 & 60 & 10 & 10 & 0 & 50 & $93 \%$ & $26 \%$ & $56 \%$ \\
\hline 17.04 & 7 & 25,8 & 19,2 & 33,3 & 21,7 & 50 & 60 & 70 & 60 & 30 & 30 & 70 & 30 & 50 & $60 \%$ & $42 \%$ & $50 \%$ \\
\hline 17.04 & 8 & 41,7 & 6,7 & 15,0 & 36,7 & 30 & 20 & 50 & 50 & 50 & 10 & 10 & 5 & 15 & $38 \%$ & $18 \%$ & $27 \%$ \\
\hline 17.05 & 1 & 25,0 & 20,0 & 23,3 & 31,7 & 50 & 30 & 50 & 50 & 30 & 50 & 30 & 60 & 70 & $45 \%$ & $48 \%$ & $47 \%$ \\
\hline 17.05 & 2 & n.b. & n.b. & n.b. & n.b. & 60 & 50 & 50 & 50 & 50 & 70 & 50 & 40 & 60 & $53 \%$ & $54 \%$ & $53 \%$ \\
\hline 17.05 & 3 & 28,3 & 25,0 & 16,7 & 30,0 & 30 & 30 & 50 & 30 & 10 & 20 & 10 & 10 & 20 & $35 \%$ & $14 \%$ & $23 \%$ \\
\hline 17.05 & 4 & 33,3 & 15,0 & 11,7 & 40,0 & 40 & 10 & 50 & 50 & 40 & 20 & 30 & 80 & 30 & $38 \%$ & $40 \%$ & $39 \%$ \\
\hline 17.05 & 5 & 56,7 & 10,0 & 14,2 & 19,2 & 20 & 60 & 80 & 80 & 80 & 80 & 30 & 70 & 80 & $60 \%$ & $68 \%$ & $64 \%$ \\
\hline 17.06 & 1 & 25,0 & 27,5 & 24,2 & 23,3 & 40 & 30 & 60 & 50 & 40 & 30 & 50 & 30 & 50 & $45 \%$ & $40 \%$ & $42 \%$ \\
\hline 17.06 & 2 & 33,3 & 33,3 & 15,8 & 17,5 & 90 & 80 & 60 & 80 & 70 & 50 & 60 & 70 & 50 & $78 \%$ & $60 \%$ & $68 \%$ \\
\hline 17.06 & 3 & 26,7 & 5,8 & 19,2 & 48,3 & 20 & 40 & 40 & 30 & 20 & 30 & 40 & 50 & 70 & $33 \%$ & $42 \%$ & $38 \%$ \\
\hline 17.06 & 4 & 33,3 & 20,0 & 20,0 & 26,7 & 20 & 80 & 80 & 80 & 80 & 60 & 80 & 80 & 70 & $65 \%$ & $74 \%$ & $70 \%$ \\
\hline 17.06 & 5 & 24,2 & 10,8 & 30,8 & 34,2 & 30 & 60 & 50 & 50 & 20 & 20 & 30 & 10 & 30 & $48 \%$ & $22 \%$ & $33 \%$ \\
\hline 17.07 & 1 & 31,7 & 25,0 & 20,0 & 23,3 & 30 & 40 & 50 & 50 & 30 & 10 & 20 & 10 & 40 & $43 \%$ & $22 \%$ & $31 \%$ \\
\hline 17.07 & 2 & 36,7 & 26,7 & 20,0 & 16,7 & 50 & 50 & 70 & 80 & 80 & 70 & 50 & 50 & 50 & $63 \%$ & $60 \%$ & $61 \%$ \\
\hline
\end{tabular}




\begin{tabular}{|c|c|c|c|c|c|c|c|c|c|c|c|c|c|c|c|c|c|}
\hline \multicolumn{2}{|c|}{ Extract } & \multicolumn{4}{|c|}{ Culture } & \multicolumn{9}{|c|}{ Informal organization } & \multirow[b]{2}{*}{ Soft } & \multirow{2}{*}{\multicolumn{2}{|c|}{ Hard Informa }} \\
\hline Case & \#P & Clan & Adh & Market & Hierarch & CI & Lead & Part & Trst & F\&R & FN & Deci & Plan & Mon & & & \\
\hline 17.07 & 3 & 31,7 & 28,3 & 21,7 & 18,3 & 60 & 50 & 70 & 50 & 30 & 50 & 50 & 30 & 30 & $58 \%$ & $38 \%$ & $47 \%$ \\
\hline 17.07 & 4 & 47,5 & 23,3 & 17,5 & 11,7 & 50 & 50 & 70 & 50 & 0 & 70 & 0 & 20 & 50 & $55 \%$ & $28 \%$ & $40 \%$ \\
\hline 17.07 & 5 & 40,0 & 20,8 & 15,8 & 23,3 & 40 & 50 & 70 & 50 & 70 & 50 & 60 & 70 & 50 & $53 \%$ & $60 \%$ & $57 \%$ \\
\hline 17.08 & 1 & 46,7 & 23,3 & 13,3 & 16,7 & 60 & 40 & 70 & 30 & 70 & 50 & 30 & 40 & 60 & $50 \%$ & $50 \%$ & $50 \%$ \\
\hline 17.08 & 2 & 44,2 & 32,5 & 6,7 & 16,7 & 80 & 60 & 70 & 70 & 30 & 30 & 50 & 50 & 50 & $70 \%$ & $42 \%$ & $54 \%$ \\
\hline 17.08 & 3 & 35,0 & 25,0 & 13,3 & 26,7 & 60 & 50 & 60 & 50 & 20 & 20 & 30 & 60 & 20 & $55 \%$ & $30 \%$ & $41 \%$ \\
\hline 17.08 & 4 & 25,0 & 13,3 & 29,2 & 32,5 & 60 & 40 & 60 & 60 & 40 & 60 & 20 & 70 & 80 & $55 \%$ & $54 \%$ & $54 \%$ \\
\hline 17.08 & 5 & 30,0 & 28,3 & 21,7 & 20,0 & 60 & 40 & 80 & 50 & 30 & 10 & 10 & 60 & 40 & $58 \%$ & $30 \%$ & $42 \%$ \\
\hline 17.08 & 6 & 25,0 & 20,0 & 26,7 & 28,3 & 70 & 30 & 50 & 30 & 20 & 20 & 10 & 20 & 20 & $45 \%$ & $18 \%$ & $30 \%$ \\
\hline 17.08 & 7 & 36,7 & 11,7 & 18,3 & 33,3 & 20 & 20 & 70 & 60 & 60 & 50 & 50 & 70 & 70 & $43 \%$ & $60 \%$ & $52 \%$ \\
\hline 17.09 & 1 & 33,3 & 23,3 & 8,3 & 35,0 & 70 & 50 & 80 & 50 & 10 & 70 & 10 & 50 & 60 & $63 \%$ & $40 \%$ & $50 \%$ \\
\hline 17.09 & 2 & 38,3 & 20,0 & 5,8 & 35,8 & 50 & 30 & 70 & 30 & 40 & 30 & 40 & 60 & 60 & $45 \%$ & $46 \%$ & $46 \%$ \\
\hline 17.09 & 3 & 24,2 & 25,0 & 10,0 & 40,8 & 30 & 40 & 30 & 30 & 20 & 35 & 10 & 20 & 20 & $33 \%$ & $21 \%$ & $26 \%$ \\
\hline 17.09 & 4 & 30,5 & 28,0 & 15,3 & 26,2 & 30 & 50 & 60 & 30 & 20 & 30 & 30 & 30 & 40 & $43 \%$ & $30 \%$ & $36 \%$ \\
\hline 17.09 & 5 & 60,0 & 23,4 & 8,4 & 8,4 & 50 & 30 & 50 & 50 & 10 & 30 & 50 & 60 & 50 & $45 \%$ & $40 \%$ & $42 \%$ \\
\hline 17.09 & 6 & 31,7 & 23,3 & 26,7 & 18,3 & 60 & 80 & 70 & 70 & 40 & 50 & 30 & 50 & 40 & $70 \%$ & $42 \%$ & $54 \%$ \\
\hline 17.09 & 7 & 17,5 & 12,4 & 27,4 & 42,4 & 40 & 30 & 50 & 30 & 40 & 20 & 20 & 40 & 30 & $38 \%$ & $30 \%$ & $33 \%$ \\
\hline 17.10 & 1 & 26,7 & 20,0 & 21,7 & 31,7 & 50 & 50 & 50 & 60 & 40 & 20 & 40 & 20 & 30 & $53 \%$ & $30 \%$ & $40 \%$ \\
\hline 17.10 & 2 & 38,0 & 15,0 & 16,0 & 31,0 & 50 & 40 & 60 & 50 & 40 & 30 & 40 & 30 & 30 & $50 \%$ & $34 \%$ & $41 \%$ \\
\hline 17.10 & 3 & 35,0 & 0,0 & 1,7 & 63,3 & 70 & 30 & 50 & 50 & 10 & 10 & 20 & 10 & 30 & $50 \%$ & $16 \%$ & $31 \%$ \\
\hline 17.10 & 4 & 38,3 & 19,2 & 24,2 & 18,3 & 60 & 50 & 70 & 70 & 80 & 70 & 60 & 30 & 20 & $63 \%$ & $52 \%$ & $57 \%$ \\
\hline 17.10 & 5 & 48,0 & 5,0 & 3,0 & 44,0 & 50 & 60 & 70 & 80 & 30 & 40 & 70 & 30 & 50 & $65 \%$ & $44 \%$ & $53 \%$ \\
\hline 17.10 & 6 & 31,7 & 25,0 & 21,7 & 21,7 & 50 & 40 & 70 & 40 & 30 & 40 & 40 & 40 & 50 & $50 \%$ & $40 \%$ & $44 \%$ \\
\hline 17.10 & 7 & 43,3 & 10,0 & 11,7 & 35,0 & 50 & 30 & 60 & 50 & 30 & 50 & 40 & 20 & 30 & $48 \%$ & $34 \%$ & $40 \%$ \\
\hline 17.10 & 8 & 25,0 & 20,8 & 15,8 & 38,3 & 60 & 40 & 70 & 50 & 60 & 30 & 50 & 60 & 50 & $55 \%$ & $50 \%$ & $52 \%$ \\
\hline 17.10 & 9 & 40,5 & 20,0 & 18,3 & 21,2 & 70 & 60 & 70 & 70 & 40 & 40 & 40 & 60 & 60 & $68 \%$ & $48 \%$ & $57 \%$ \\
\hline \multirow[t]{2}{*}{17.10} & 10 & 31,7 & 0,0 & 0,0 & 68,3 & 60 & 30 & 60 & 40 & 40 & 60 & 40 & 25 & 25 & $48 \%$ & $38 \%$ & $42 \%$ \\
\hline & 60 & \multicolumn{4}{|c|}{ Participants } & & & & & & & & & & & & \\
\hline \multirow[t]{2}{*}{2017} & Min. & 15,8 & 0,0 & 0,0 & 2,5 & 10 & 10 & 30 & 30 & 0 & 10 & 0 & 0 & 15 & $33 \%$ & $14 \%$ & $23 \%$ \\
\hline & Max. & 65,8 & 35,0 & 33,3 & 68,3 & 90 & 100 & 100 & 90 & 100 & 100 & 80 & 100 & 100 & $93 \%$ & $90 \%$ & $71 \%$ \\
\hline
\end{tabular}

Table 82 Results case studies 2017, context (department view, end result) 


\begin{tabular}{|c|c|c|c|c|c|c|c|c|c|c|c|}
\hline \multicolumn{3}{|c|}{ Extract from case study database v. 0.82} & \multicolumn{9}{|c|}{ View: Organization } \\
\hline Case & \#P & Function & CI & Lead & Part & Trst & F\&R & FN & Deci & Plan & Mon \\
\hline 17.01 & 1 & Service Process Consultant & A & $\mathrm{A}$ & $\mathrm{C}$ & A & A & A & A & A & A \\
\hline 17.01 & 2 & Program Manager Lean IT transformation & $\mathrm{CI}$ & $\mathrm{F}$ & B & B & A & A & $\mathrm{C}$ & $\mathrm{C}$ & $\mathrm{E}$ \\
\hline 17.01 & 3 & Teamlead IT & A & E & B & A & A & $\mathrm{C}$ & $\mathrm{D}$ & A & $\mathrm{C}$ \\
\hline 17.01 & 4 & Manager & A & A & A & A & A & A & A & B & B \\
\hline 17.02 & 1 & n.b. & & & & & & & & & \\
\hline 17.02 & 2 & n.b. & A & A & A & A & A & A & A & A & A \\
\hline 17.02 & 3 & n.b. & A & E & A & A & $\mathrm{C}$ & B & A & A & $\mathrm{C}$ \\
\hline 17.02 & 4 & n.b. & A & $\mathrm{E}$ & A & A & $\mathrm{C}$ & A & A & $\mathrm{C}$ & A \\
\hline 17.02 & 5 & n.b. & A & A & A & A & A & $\mathrm{A}$ & A & A & $\mathrm{A}$ \\
\hline 17.03 & 1 & Global Head Lending \& Trade & A & $\mathrm{E}$ & A & A & $\mathrm{C}$ & B & A & A & A \\
\hline 17.03 & 2 & IT manager & A & B & A & A & A & A & A & A & $\mathrm{A}$ \\
\hline 17.03 & 3 & Manager & A & A & A & A & A & A & A & A & $\mathrm{C}$ \\
\hline 17.03 & 4 & Student group & $\mathrm{CI}$ & A & $\mathrm{A}$ & A & $\mathrm{D}$ & $\mathrm{E}$ & $\mathrm{D}$ & $\mathrm{C}$ & $\mathrm{C}$ \\
\hline 17.04 & 1 & E-commerce Business Process Manager & A & A & A & A & A & A & A & A & A \\
\hline 17.04 & 2 & iOS Developer & $\mathrm{D}$ & $\mathrm{F}$ & $\mathrm{C}$ & A & A & A & E & $\mathrm{D}$ & $\mathrm{A}$ \\
\hline 17.04 & 3 & Coorinator & A & $\mathrm{F}$ & A & A & $\mathrm{D}$ & A & $\mathrm{E}$ & $\mathrm{E}$ & $\mathrm{E}$ \\
\hline 17.04 & 4 & iOS Developer & $\mathrm{D}$ & $\mathrm{F}$ & B & A & $\mathrm{E}$ & B & $\mathrm{E}$ & A & $\mathrm{E}$ \\
\hline 17.04 & 5 & Assistent Engineer & $\mathrm{E}$ & $\mathrm{F}$ & A & A & B & $\mathrm{A}$ & A & $\mathrm{E}$ & $\mathrm{A}$ \\
\hline 17.04 & 6 & Backend Developer & $\mathrm{A}$ & $\mathrm{F}$ & A & A & $\mathrm{A}$ & $\mathrm{A}$ & A & A & $\mathrm{A}$ \\
\hline 17.04 & 7 & Team lead/scrum master & $\mathrm{A}$ & A & A & A & $\mathrm{A}$ & $\mathrm{A}$ & A & A & $\mathrm{A}$ \\
\hline 17.04 & 8 & Mobile Engineer & $\mathrm{A}$ & A & $\mathrm{A}$ & A & $\mathrm{A}$ & $\mathrm{A}$ & A & A & $\mathrm{A}$ \\
\hline 17.05 & 1 & Manager TCE-NL & $\mathrm{A}$ & $\mathrm{F}$ & A & A & $\mathrm{A}$ & $\mathrm{D}$ & $\mathrm{E}$ & $\mathrm{E}$ & $\mathrm{E}$ \\
\hline 17.05 & 2 & Manager sw engineering team & B & A & $\mathrm{D}$ & A & $\mathrm{E}$ & $\mathrm{A}$ & $\mathrm{E}$ & $\mathrm{E}$ & $\mathrm{E}$ \\
\hline 17.05 & 3 & IS/IT Manager & $\mathrm{A}$ & A & B & A & A & $\mathrm{A}$ & A & A & $\mathrm{A}$ \\
\hline 17.05 & 4 & IS/IT Solution Engineer & $\mathrm{A}$ & $\mathrm{E}$ & A & A & $\mathrm{A}$ & $\mathrm{E}$ & $\mathrm{C}$ & A & $\mathrm{A}$ \\
\hline 17.05 & 5 & Spares \& Repairs Manager & $\mathrm{A}$ & B & A & A & $\mathrm{A}$ & $\mathrm{A}$ & A & A & A \\
\hline 17.06 & 1 & Warehouse Supervisor & $\mathrm{A}$ & A & A & A & A & A & A & A & $\mathrm{E}$ \\
\hline 17.06 & 2 & E-shop manager & $\mathrm{A}$ & B & A & A & $\mathrm{A}$ & $\mathrm{A}$ & A & A & A \\
\hline 17.06 & 3 & Order picker & $\mathrm{A}$ & A & $\mathrm{A}$ & A & $\mathrm{A}$ & A & A & A & A \\
\hline 17.06 & 4 & Production employee ICT department & $\mathrm{A}$ & A & A & A & $\mathrm{A}$ & $\mathrm{A}$ & A & A & A \\
\hline 17.06 & 5 & Order picker & $\mathrm{A}$ & $\mathrm{C}$ & B & A & B & A & A & $\mathrm{E}$ & $\mathrm{A}$ \\
\hline 17.07 & 1 & Consultant & A & A & A & A & A & A & A & A & A \\
\hline
\end{tabular}




\begin{tabular}{|c|c|c|c|c|c|c|c|c|c|c|c|}
\hline \multicolumn{3}{|c|}{ Extract from case study database v. 0.82} & \multicolumn{9}{|c|}{ View: Organization } \\
\hline Case & \#P & Function & $\mathrm{CI}$ & Lead & Part & Trst & F\&R & FN & Deci & Plan & Mon \\
\hline 17.07 & 2 & Business Unit Manager & $\mathrm{E}$ & B & A & A & A & $\mathrm{E}$ & $\mathrm{E}$ & $\mathrm{E}$ & $\mathrm{E}$ \\
\hline 17.07 & 3 & Consultant & A & $\mathrm{D}$ & A & $\mathrm{A}$ & $\mathrm{A}$ & A & $\mathrm{A}$ & $\mathrm{A}$ & A \\
\hline 17.07 & 4 & Consultant & $\mathrm{E}$ & $\mathrm{A}$ & $\mathrm{A}$ & B & $\mathrm{E}$ & A & A & $\mathrm{E}$ & A \\
\hline 17.07 & 5 & Business Manager & $\mathrm{D}$ & $\mathrm{F}$ & $\mathrm{A}$ & $\mathrm{A}$ & $\mathrm{E}$ & $\mathrm{E}$ & $\mathrm{E}$ & $\mathrm{E}$ & E \\
\hline 17.08 & 1 & Team coordinator SAP & A & $\mathrm{A}$ & A & A & $\mathrm{A}$ & A & $\mathrm{A}$ & A & $\mathrm{E}$ \\
\hline 17.08 & 2 & Coordinator technical application design & A & A & $\mathrm{A}$ & A & $\mathrm{D}$ & $\mathrm{D}$ & A & A & $\mathrm{E}$ \\
\hline 17.08 & 3 & Technical coordinator & A & $\mathrm{E}$ & A & A & $\mathrm{D}$ & A & A & A & $\mathrm{E}$ \\
\hline 17.08 & 4 & Enterprise Architect & A & A & A & $\mathrm{A}$ & $\mathrm{A}$ & A & $\mathrm{A}$ & A & A \\
\hline 17.08 & 5 & Teamcoordinator ICT beheer \& helpdesk & A & A & $\mathrm{C}$ & A & $\mathrm{C}$ & A & A & A & A \\
\hline 17.08 & 6 & ICT manager (CIO) & A & $\mathrm{E}$ & $\mathrm{A}$ & $\mathrm{A}$ & A & A & A & A & A \\
\hline 17.08 & 7 & Projectleider ICS & A & B & A & A & A & A & A & B & A \\
\hline 17.09 & 1 & Coordinator Enterprise Architecture & A & B & $\mathrm{A}$ & A & $\mathrm{A}$ & B & $\mathrm{A}$ & B & B \\
\hline 17.09 & 2 & Senior adviseur Informatisering & A & $\mathrm{C}$ & $\mathrm{A}$ & $\mathrm{A}$ & A & $\mathrm{C}$ & A & B & A \\
\hline 17.09 & 3 & Head Risc manager & B & $\mathrm{C}$ & B & B & $\mathrm{C}$ & $\mathrm{C}$ & $\mathrm{C}$ & $\mathrm{C}$ & $\mathrm{C}$ \\
\hline 17.09 & 4 & Senior projectleader & A & B & $\mathrm{A}$ & B & B & A & $\mathrm{A}$ & B & A \\
\hline 17.09 & 5 & $\begin{array}{l}\text { Manager program management } \\
\text { department }\end{array}$ & A & B & B & $\mathrm{C}$ & B & B & $\mathrm{B}$ & A & B \\
\hline 17.09 & 6 & Department manager & A & $\mathrm{F}$ & A & $\mathrm{A}$ & $\mathrm{D}$ & $\mathrm{C}$ & A & A & $\mathrm{C}$ \\
\hline 17.09 & 7 & Coördinerend beleidsmedewerker & $\mathrm{C}$ & A & $\mathrm{A}$ & A & $\mathrm{A}$ & B & $\mathrm{C}$ & B & A \\
\hline 17.10 & 1 & Strategisch adviseur & A & A & A & A & B & $\mathrm{C}$ & A & $\mathrm{E}$ & A \\
\hline 17.10 & 2 & Project manager & A & A & $\mathrm{A}$ & A & $\mathrm{A}$ & A & A & A & A \\
\hline 17.10 & 3 & Manager Service Delivery & A & B & B & A & $\mathrm{C}$ & $\mathrm{C}$ & $\mathrm{C}$ & $\mathrm{C}$ & $\mathrm{C}$ \\
\hline 17.10 & 4 & Functional designer & A & B & B & B & $\mathrm{C}$ & A & A & $\mathrm{C}$ & $\mathrm{C}$ \\
\hline 17.10 & 5 & Klant Domein Manager & A & $\mathrm{C}$ & B & A & $\mathrm{C}$ & A & A & A & A \\
\hline 17.10 & 6 & Manager & $\mathrm{C}$ & $\mathrm{D}$ & B & A & $\mathrm{C}$ & A & A & A & B \\
\hline 17.10 & 7 & Vice Director information management & A & A & B & A & A & A & A & A & A \\
\hline 17.10 & 8 & Lead Architect OB & A & $\mathrm{A}$ & A & $\mathrm{B}$ & $\mathrm{B}$ & $\mathrm{C}$ & $\mathrm{A}$ & A & $\mathrm{A}$ \\
\hline 17.10 & 9 & Project Manager \& Product Manager & B & B & $\mathrm{C}$ & A & $\mathrm{C}$ & $\mathrm{C}$ & A & $\mathrm{C}$ & $\mathrm{C}$ \\
\hline \multirow[t]{2}{*}{17.10} & 10 & Projectmanager - Release train engineer & A & A & B & A & $\mathrm{A}$ & A & B & $\mathrm{C}$ & $\mathrm{E}$ \\
\hline & 60 & Participants & & & & & & & & & \\
\hline \multirow[t]{2}{*}{2017} & 10 & Cases & & & & & & & & & \\
\hline & 60 & Participants & & & & & & & & & \\
\hline
\end{tabular}

Table 83 Results case studies 2017, hard and soft governance (organisation view, end result) 


\begin{tabular}{|c|c|c|c|c|c|c|c|c|c|c|c|c|c|c|c|c|c|}
\hline \multicolumn{2}{|c|}{ Extract } & \multicolumn{4}{|c|}{ Culture } & \multicolumn{9}{|c|}{ Informal organization } & \multirow[b]{2}{*}{ Soft } & \multirow[b]{2}{*}{ Hard } & \multirow[b]{2}{*}{ Informal } \\
\hline Case & \#P & Clan & Adh & Market & Hierarch & CI & Lead & Part & Trst & F\&R & FN & Deci & Plan & Mon & & & \\
\hline 17.01 & 1 & 13,3 & 18,3 & 31,7 & 36,7 & 40 & 30 & 30 & 40 & 30 & 30 & 20 & 20 & 30 & $35 \%$ & $26 \%$ & $30 \%$ \\
\hline 17.01 & 2 & 30,0 & 23,3 & 25,0 & 21,7 & 40 & 60 & 70 & 50 & 40 & 40 & 50 & 20 & 50 & $55 \%$ & $40 \%$ & $47 \%$ \\
\hline 17.01 & 3 & 25,0 & 30,0 & 26,7 & 18,3 & 30 & 30 & 70 & 60 & 10 & 20 & 10 & 40 & 40 & $48 \%$ & $24 \%$ & $34 \%$ \\
\hline 17.01 & 4 & 21,7 & 20,0 & 41,7 & 16,7 & 40 & 40 & 80 & 30 & 50 & 50 & 50 & 50 & 40 & $48 \%$ & $48 \%$ & $48 \%$ \\
\hline 17.02 & 1 & & & & & & & & & & & & & & & & \\
\hline 17.02 & 2 & 18,3 & 6,7 & 25,0 & 50,0 & 60 & 20 & 40 & 50 & 10 & 50 & 10 & 40 & 40 & $43 \%$ & $30 \%$ & $36 \%$ \\
\hline 17.02 & 3 & 15,0 & 29,2 & 30,0 & 25,8 & 30 & 80 & 60 & 50 & 20 & 50 & 80 & 80 & 30 & $55 \%$ & $52 \%$ & $53 \%$ \\
\hline 17.02 & 4 & 41,7 & 15,0 & 13,3 & 30,0 & n.b. & 50 & 40 & 50 & 30 & 20 & 20 & 40 & 30 & $47 \%$ & $28 \%$ & $35 \%$ \\
\hline 17.02 & 5 & 20,8 & 18,3 & 10,0 & 50,8 & 20 & 20 & 80 & 60 & 30 & 30 & 40 & 30 & 30 & $45 \%$ & $32 \%$ & $38 \%$ \\
\hline 17.03 & 1 & 6,7 & 21,7 & 40,0 & 31,7 & 60 & 20 & 90 & 30 & 10 & 10 & 10 & 80 & 20 & $50 \%$ & $26 \%$ & $37 \%$ \\
\hline 17.03 & 2 & 5,0 & 25,0 & 45,0 & 25,0 & n.b. & n.b. & n.b. & 50 & 30 & 20 & 20 & 20 & 20 & $50 \%$ & $22 \%$ & $18 \%$ \\
\hline 17.03 & 3 & 26,7 & 21,7 & 13,3 & 38,3 & 20 & 10 & 70 & 70 & 70 & 50 & 60 & 20 & 90 & $43 \%$ & $58 \%$ & $51 \%$ \\
\hline 17.03 & 4 & 12,5 & 23,3 & 37,5 & 26,7 & 60 & 20 & 60 & 20 & 30 & 10 & 50 & 30 & 20 & $40 \%$ & $28 \%$ & $33 \%$ \\
\hline 17.04 & 1 & 35,8 & 28,3 & 23,3 & 12,5 & 40 & 40 & 70 & 70 & 80 & 70 & 50 & 30 & 50 & $55 \%$ & $56 \%$ & $56 \%$ \\
\hline 17.04 & 2 & 41,7 & 25,0 & 10,0 & 23,3 & 70 & 20 & 40 & 50 & 30 & 50 & 25 & 30 & 30 & $45 \%$ & $33 \%$ & $38 \%$ \\
\hline 17.04 & 3 & 65,8 & 27,5 & 4,2 & 2,5 & 60 & 60 & 60 & 60 & 60 & 60 & 60 & 60 & 60 & $60 \%$ & $60 \%$ & $60 \%$ \\
\hline 17.04 & 4 & 30,0 & 25,0 & 18,3 & 26,7 & 40 & 30 & 40 & 30 & 20 & 40 & 10 & 10 & 20 & $35 \%$ & $20 \%$ & $27 \%$ \\
\hline 17.04 & 5 & 41,7 & 37,5 & 13,3 & 7,5 & 50 & 40 & 50 & 60 & 60 & 60 & 40 & 40 & 40 & $50 \%$ & $48 \%$ & $49 \%$ \\
\hline 17.04 & 6 & 21,7 & 22,5 & 34,2 & 21,7 & 80 & 80 & 90 & 80 & 70 & 10 & 10 & 0 & 50 & $83 \%$ & $28 \%$ & $52 \%$ \\
\hline 17.04 & 7 & 25,8 & 19,2 & 33,3 & 21,7 & 50 & 60 & 70 & 60 & 30 & 30 & 70 & 30 & 50 & $60 \%$ & $42 \%$ & $50 \%$ \\
\hline 17.04 & 8 & 41,7 & 6,7 & 15,0 & 36,7 & 30 & 20 & 50 & 50 & 50 & 10 & 10 & 5 & 15 & $38 \%$ & $18 \%$ & $27 \%$ \\
\hline 17.05 & 1 & 23,3 & 28,3 & 28,3 & 20,0 & 60 & 50 & 30 & 50 & 30 & 40 & 50 & 40 & 50 & $48 \%$ & $42 \%$ & $44 \%$ \\
\hline 17.05 & 2 & n.b. & n.b. & n.b. & n.b. & 30 & 40 & 30 & 50 & 50 & 50 & 50 & 40 & 30 & $38 \%$ & $44 \%$ & $41 \%$ \\
\hline 17.05 & 3 & n.b. & n.b. & n.b. & n.b. & 10 & 30 & 30 & 30 & 10 & n.b. & n.b. & n.b. & n.b. & $25 \%$ & $10 \%$ & $22 \%$ \\
\hline 17.05 & 4 & 31,7 & 15,0 & 18,3 & 35,0 & 20 & 10 & 50 & 50 & 40 & 20 & 40 & 60 & 30 & $33 \%$ & $38 \%$ & $36 \%$ \\
\hline 17.05 & 5 & 60,0 & 6,7 & 12,5 & 20,8 & 20 & 20 & 80 & 40 & 40 & 40 & 20 & 70 & 80 & $40 \%$ & $50 \%$ & $46 \%$ \\
\hline 17.06 & 1 & 20,0 & 30,0 & 28,3 & 21,7 & 30 & 20 & 60 & 50 & 40 & 30 & 50 & 20 & 40 & $40 \%$ & $36 \%$ & $38 \%$ \\
\hline 17.06 & 2 & 31,7 & 25,0 & 19,2 & 24,2 & 40 & 50 & 40 & 70 & 70 & 80 & 30 & 30 & 80 & $50 \%$ & $58 \%$ & $54 \%$ \\
\hline 17.06 & 3 & 40,0 & 21,7 & 15,0 & 23,3 & 50 & 30 & 30 & 30 & 10 & 20 & 30 & 30 & 30 & $35 \%$ & $24 \%$ & $29 \%$ \\
\hline 17.06 & 4 & 20,0 & 21,7 & 36,7 & 21,7 & 80 & 30 & 80 & 80 & 20 & 60 & 30 & 60 & 30 & $68 \%$ & $40 \%$ & $52 \%$ \\
\hline 17.06 & 5 & 30,0 & 28,3 & 24,2 & 17,5 & 50 & 50 & 50 & 50 & 70 & 80 & 60 & 70 & 70 & $50 \%$ & $70 \%$ & $61 \%$ \\
\hline 17.07 & 1 & 28,3 & 21,7 & 26,7 & 23,3 & 50 & 40 & 40 & 50 & 20 & 10 & 20 & 10 & 40 & $45 \%$ & $20 \%$ & $31 \%$ \\
\hline
\end{tabular}




\begin{tabular}{|c|c|c|c|c|c|c|c|c|c|c|c|c|c|c|c|c|c|}
\hline \multicolumn{2}{|c|}{ Extract } & \multicolumn{4}{|c|}{ Culture } & \multicolumn{9}{|c|}{ Informal organization } & \multirow[b]{2}{*}{ Soft } & \multirow{2}{*}{\multicolumn{2}{|c|}{ Hard Informal }} \\
\hline Case & \#P & Clan & Adh & Market & Hierarch & CI & Lead & Part & Trst & F\&R & FN & Deci & Plan & Mon & & & \\
\hline 17.07 & 2 & 36,7 & 26,7 & 20,0 & 16,7 & 50 & 50 & 70 & 80 & 80 & 70 & 50 & 50 & 50 & $63 \%$ & $60 \%$ & $61 \%$ \\
\hline 17.07 & 3 & 31,7 & 28,3 & 21,7 & 18,3 & 60 & 50 & 70 & 50 & 30 & 50 & 50 & 30 & 30 & $58 \%$ & $38 \%$ & $47 \%$ \\
\hline 17.07 & 4 & 40,7 & 28,0 & 17,8 & 13,6 & 50 & 50 & 80 & 50 & 0 & 70 & 0 & 10 & 50 & $58 \%$ & $26 \%$ & $40 \%$ \\
\hline 17.07 & 5 & 26,7 & 21,7 & 27,5 & 24,2 & 40 & 30 & 60 & 50 & 40 & 50 & 10 & 40 & 50 & $45 \%$ & $38 \%$ & $41 \%$ \\
\hline 17.08 & 1 & 41,7 & 23,3 & 15,0 & 20,0 & 30 & 30 & 60 & 30 & 70 & 50 & 30 & 40 & 60 & $38 \%$ & $50 \%$ & $44 \%$ \\
\hline 17.08 & 2 & 36,7 & 8,3 & 23,3 & 31,7 & 30 & 20 & 50 & 50 & 50 & 10 & 20 & 30 & 40 & $38 \%$ & $30 \%$ & $33 \%$ \\
\hline 17.08 & 3 & 38,3 & 16,7 & 23,3 & 21,7 & 20 & 10 & 20 & 30 & 10 & 20 & 40 & 10 & 20 & $20 \%$ & $20 \%$ & $20 \%$ \\
\hline 17.08 & 4 & 29,2 & 15,0 & 31,7 & 24,2 & 70 & 30 & 70 & 70 & 40 & 60 & 70 & 80 & 80 & $60 \%$ & $66 \%$ & $63 \%$ \\
\hline 17.08 & 5 & 16,7 & 21,7 & 33,3 & 28,3 & 40 & 20 & 20 & 30 & 20 & 10 & 20 & 20 & 40 & $28 \%$ & $22 \%$ & $24 \%$ \\
\hline 17.08 & 6 & 19,2 & 13,3 & 45,0 & 22,5 & 70 & 20 & 60 & 30 & 30 & 30 & 30 & 30 & 30 & $45 \%$ & $30 \%$ & $37 \%$ \\
\hline 17.08 & 7 & 25,8 & 14,2 & 28,3 & 31,7 & 30 & 20 & 60 & 40 & 30 & 30 & 30 & 50 & 40 & $38 \%$ & $36 \%$ & $37 \%$ \\
\hline 17.09 & 1 & 19,2 & 9,2 & 10,0 & 61,7 & 90 & 70 & 80 & 70 & 10 & 90 & 10 & 50 & 70 & $78 \%$ & $46 \%$ & $60 \%$ \\
\hline 17.09 & 2 & 32,5 & 15,0 & 5,0 & 47,5 & 40 & 30 & 50 & 40 & 20 & 10 & 20 & 60 & 40 & $40 \%$ & $30 \%$ & $34 \%$ \\
\hline 17.09 & 3 & 29,2 & 17,5 & 10,0 & 43,3 & 20 & 50 & 20 & 20 & 15 & 30 & 10 & 20 & 20 & $28 \%$ & $19 \%$ & $23 \%$ \\
\hline 17.09 & 4 & 30,8 & 24,2 & 15,0 & 30,0 & 30 & 40 & 70 & 20 & 20 & 30 & 30 & 30 & 40 & $40 \%$ & $30 \%$ & $34 \%$ \\
\hline 17.09 & 5 & 20,0 & 18,4 & 18,4 & 43,4 & 50 & 20 & 50 & 50 & 10 & 20 & 50 & 60 & 50 & $43 \%$ & $38 \%$ & $40 \%$ \\
\hline 17.09 & 6 & 20,0 & 15,8 & 37,5 & 26,7 & 70 & 80 & 70 & 70 & 30 & 40 & 50 & 50 & 30 & $73 \%$ & $40 \%$ & $54 \%$ \\
\hline 17.09 & 7 & 12,2 & 12,2 & 24,2 & 51,5 & 30 & 20 & 30 & 20 & 20 & 20 & 20 & 30 & 20 & $25 \%$ & $22 \%$ & $23 \%$ \\
\hline 17.10 & 1 & 20,0 & 18,3 & 23,3 & 38,3 & 40 & 40 & 30 & 50 & 40 & 20 & 30 & 50 & 20 & $40 \%$ & $32 \%$ & $36 \%$ \\
\hline 17.10 & 2 & 31,0 & 15,0 & 18,0 & 36,0 & 40 & 40 & 40 & 60 & 30 & 20 & 30 & 20 & 20 & $45 \%$ & $24 \%$ & $33 \%$ \\
\hline 17.10 & 3 & 16,7 & 0,0 & 0,0 & 83,3 & 20 & 10 & 30 & 50 & 10 & 10 & 10 & 10 & 10 & $28 \%$ & $10 \%$ & $18 \%$ \\
\hline 17.10 & 4 & 31,7 & 24,2 & 20,0 & 24,2 & 40 & 30 & 50 & 50 & 50 & 50 & 50 & 30 & 50 & $43 \%$ & $46 \%$ & $44 \%$ \\
\hline 17.10 & 5 & n.b. & & & & 50 & 40 & 60 & 80 & 30 & 30 & 60 & 30 & 30 & $58 \%$ & $36 \%$ & $46 \%$ \\
\hline 17.10 & 6 & 28,3 & 15,0 & 28,3 & 28,3 & 40 & 40 & 60 & 40 & 20 & 30 & 30 & 40 & 40 & $45 \%$ & $32 \%$ & $38 \%$ \\
\hline 17.10 & 7 & 43,3 & 10,0 & 11,7 & 35,0 & 50 & 30 & 60 & 50 & 30 & 50 & 40 & 20 & 30 & $48 \%$ & $34 \%$ & $40 \%$ \\
\hline 17.10 & 8 & 20,0 & 14,2 & 17,5 & 48,3 & 80 & 40 & 80 & 50 & 60 & 20 & 40 & 50 & 50 & $63 \%$ & $44 \%$ & $52 \%$ \\
\hline 17.10 & 9 & 39,2 & 19,2 & 17,5 & 24,2 & 70 & 65 & 60 & 80 & 40 & 50 & 50 & 60 & 50 & $69 \%$ & $50 \%$ & $58 \%$ \\
\hline \multirow[t]{2}{*}{17.10} & 10 & 23,3 & 0,0 & 0,0 & 76,7 & 60 & 25 & 40 & 60 & 40 & 60 & 40 & 25 & 25 & $46 \%$ & $38 \%$ & $42 \%$ \\
\hline & 60 & \multicolumn{16}{|c|}{ Participants } \\
\hline 2017 & $\min$. & 5,0 & 0,0 & 0,0 & 2,5 & 10 & 10 & 20 & 20 & 0 & 10 & 0 & 0 & 10 & $20 \%$ & $10 \%$ & $18 \%$ \\
\hline & $\max$. & 65,8 & 37,5 & 45,0 & 83,3 & 90 & 80 & 90 & 80 & 80 & 90 & 80 & 80 & 90 & $83 \%$ & $70 \%$ & $63 \%$ \\
\hline
\end{tabular}

Table 84 Results case studies 2017, context (organisation view, end result) 


\section{S U M M A R Y}

The goal of the research presented in this thesis is to determine how the IT governance (ITG) of an organisation can grow in maturity to become more effective.

The first ITG publications appeared in the late-1990s. While a considerable body of literature on ITG exists, definitions of ITG in the literature vary considerably. Out of a long list of ITG definitions, this research opted for the ITG definition: "the structures, process, cultures and systems that engender the successful operation of the IT of the (complete) organization". ITG is thus not restricted to the IT organisation.

A systematic literature study revealed six streams of ITG based on two views:

1. The first viewing angle handles the scope of ITG;

2. The second viewing angle handles the direction in which ITG works.

Four ITG streams differ in scope: IT audit, decision-making, "part of corporate governance, conformance perspective" and "part of corporate governance, performance perspective". The latter two streams differ in the direction in which ITG works: top-down and bottom-up.

The research presented here follows the stream in which ITG is considered an integral part of corporate governance, focusing on the performance perspective. Organisational performance is determined by a large number of elements. Trying to determine a direct relationship between ITG and organisational performance is almost impossible.

The approach is rooted in the following assumptions:

a. The proposition is that improving "ITG maturity" results in improving ITG;

b. Given that ITG is an integral part of corporate governance, the assumption is that improving ITG results in improving corporate governance;

c. Improving corporate governance results in improving organisational performance.

The main question of the research is:

[MRQ] How can the ITG of an organisation grow in maturity to become more effective?

The research in this thesis is described in sections grouped in five parts:

I. The first part consists of an introduction to the research and the research methodology. 
II. In the second part, prior research is collected, summarised and discussed with practitioners to determine the relevance and the requirements for the next phase.

III. The results of Part II will be used in the third part to design a focus area maturity model and an assessment instrument.

IV. Part IV describes three cycles in which consecutive versions of the assessment instrument are used, evaluated, validated and improved in practice, along with a systematic literature study on the relationship between corporate governance and ITG.

V. The final part (Part V) consists of the conclusions and answers to the research questions, limitations and further research.

The research methodology is based on design science and a combination of systematic literature studies, Delphi workshops and case studies. A detailed overview of the structure of this thesis is included in Section 2.4 and a research outline in Figure 6.

A Delphi workshop with a group of CIOs demonstrated how the practical relevance of the research and a systematic ITG literature review was conducted to collect prior knowledge.

The research presented in this thesis has a multi-disciplinary nature. Its roots are in information systems research but there are strong links to organisational and social theory. Organisations can be defined as social units of people that are structured and managed to pursue collective goals.

Thus, ITG can be seen from two perspectives:

- $\quad$ An organisational perspective referred to as "hard governance";

- $\quad$ A social perspective referred to as "soft governance".

This research is grounded in the assumption that in order to advance in maturity, organisations should pay attention to both the hard and soft sides of ITG. The hard side is related to processes and structure, the soft side to social aspects like behaviour and organisational culture.

The proposition was that immature or a low level of ITG maturity represent the cause of a lack of effective ITG. In order to improve ITG, a maturity model for hard and soft ITG was designed because such a model does not exist and is required in practice. This maturity model is intended to help organisations improve their hard (structure, processes) and soft (behaviour, collaboration) ITG.

In Delphi workshops with ITG experts from practice, the focus areas of an ITG maturity model for hard and soft governance were determined. During the workshops the Group Decision Support System Spilter was used. 
The end result (MIG model version 1) was a maturity model consisting of three parts: soft governance, hard governance and the context (of an organisation). The maturity part of the MIG model consists of hard and soft governance. The context is placed outside of the maturity part. The MIG model consists of four focus areas for soft governance: continuous improvement, leadership, participation, and understanding and trust. The five focus areas for hard governance comprise: functions and roles, formal networks, IT decision-making, planning and monitoring. There are three focus areas in the context: culture, informal organisation and sector. This model was named the MIG model.

The MIG model was used to design an ITG maturity model. The artefact resulting from the process was named the MIG assessment instrument. This thesis provides a detailed description of the design process between 2014 and 2017. The description of the design process starts with the initial version, the protocol for using the instrument and the changes in the second and third versions of the instrument. The design of the first version was based on the MIG model and the results of a series of Delphi workshops with practitioners.

The assessment instrument was used in case studies conducted by students and the researchers. The changes to the second and third version of the MIG assessment instrument are based on the use, improvement and validation of the instrument via several case studies. The assumption is that if students are able to use the instrument, it can be expected that practitioners - who in general have much more practical experience - will definitely be able to use the instrument.

Between 2015 and 2017, 28 case studies were conducted using the first, second and third versions of the instrument. The use of the instrument was always combined with semistructured interviews with the participant to evaluate and correct the results. The success of a maturity model is proven "if it brings about a discussion on improvement among the targeted audience" (Lasrado et al., 2017). Participants responded positively regarding the usability of the tool in general, such as "It delivers an indicative impression of the maturity of several ITG processes very quickly," or "The assessment and this interview stimulates me. In that way it has already value to me". They also proposed a large number of improvements to the instrument.

Given that five years have passed since the initial literature study, this research was repeated in 2017/2018. The update revealed that a disjuncture between ITG practice, frameworks and theory remains likely. Furthermore, five (relatively) new ITG maturity models were found (including the model that forms the subject of this thesis). Only two frameworks were found covering hard and soft ITG: COBIT 5.0 in a holistic way and the MIG model in a more practical way. 
The evaluations revealed that combining the instrument with semi-structured interviews results in an enhanced and usable instrument for determining the current level of hard and soft ITG of an organisation. The research in this thesis and the MIG assessment instrument demonstrate a means of reducing the mismatch between ITG maturity theory and practice.

Corporate governance and ITG are related and ITG can be deemed an element of corporate governance. Little is known regarding the way in which this relationship works. A systematic corporate governance literature review was conducted in 2018 to validate whether the 12 focus areas used in the MIG model exist in the contemporary corporate governance literature. This aligns with the ITG stream that defines ITG as an aspect of corporate governance. The literature review demonstrated that all 12 focus areas of the MIG model are also covered by the corporate governance literature.

This confirms that the focus areas of the MIG model are relevant for corporate governance too, and thus relevant for ITG and corporate governance. It additionally demonstrates that the "informal organisation" should be a contextual factor for corporate governance.

The contribution of the research documented in this thesis is twofold:

\section{Scientific contribution}

This thesis illustrates that for organisations, the soft side of ITG is at least as important. This goes (far) beyond relational governance mechanisms as found in current research.

In spite of the efforts made here, a causal relationship between ITG and the performance of an organisation remains unclear, as does the ITG mechanisms that affect organisational performance. The MIG model and the MIG instrument may serve as a useful model to conduct further research to develop a better understanding of these relationships.

The focus areas of the MIG model are relevant for corporate governance, too. Thus, the model and instrument might be useful for research to attain a better understanding regarding how the relationship between ITG and corporate governance works.

\section{Practical contribution}

Consultants in practice base their approach on available models, frameworks and experience. The MIG model is a model created via design science in collaboration with practitioners. This research illustrates that CIOs require the availability of a reliable measuring instrument.

The MIG instrument is a practical implementation of the MIG model and can serve as a useful tool to support employees or consultants in improving ITG in contemporary organisations. 


\section{SA M E N VAT T I N G}

Het doel van het onderzoek dat gepresenteerd wordt in deze dissertatie is om te bepalen hoe de IT governance (ITG) van een organisatie kan groeien in volwassenheid om effectiever te worden. De eerste ITG publicaties verschenen tegen het einde van de jaren 1990. Hoewel er een aanzienlijke hoeveelheid literatuur over ITG bestaat, zijn er grote verschillen van definities voor ITG in de literatuur. Uit een lange lijst van ITG definities is in dit onderzoek gekozen voor de definitie "De structuren, processen, culturen en systemen die zorgen voor het succesvolle werking van de IT van de (complete) organisatie". ITG dus, is niet beperkt tot de IT organisatie. Een systematische literatuurstudie leverde zes ITG stromingen op gebaseerd op twee gezichtspunten:

1. het eerste gezichtspunt gaat over de scope van ITG;

2. het tweede gezichtspunt gaat over de richting waarin ITG werkt.

Vier ITG stromingen verschillen in scope: IT auditing, besluitvorming, deel van corporate governance vanuit prestatie perspectief, deel van corporate governance vanuit wet- en regelgeving en twee stromingen die verschillen in de richting waarin ITG werkt: vanuit de top naar beneden en vanuit beneden naar de top.

Het onderzoek in deze dissertatie volgt de stroming waarin ITG wordt gezien als een integraal onderdeel van corporate governance waarbij de focus ligt op het performance (prestatie) perspectief. De prestatie van een organisatie wordt bepaald door een groot aantal elementen. De relatie bepalen tussen ITG en de prestatie van een organisatie is vrijwel ondoenlijk.

De aanpak van het onderzoek is gebaseerd op de volgende aannames:

a. de stelling is dat het verbeteren van de volwassenheid van ITG resulteert in het verbeteren van ITG;

b. omdat ITG een integraal deel van corporate governance is, is het uitgangspunt dat het verbeteren van ITG resulteert in het verbeteren van corporate governance;

c. het verbeteren van corporate governance resulteert in het verbeteren van de prestatie van een organisatie.

De kernvraag van het onderzoek is:

Hoe kan de ITG van een organisatie groeien in volwassenheid om effectiever te worden? 
Samenvatting

Het onderzoek in deze dissertatie is beschreven in secties die gegroepeerd zijn in vijf delen:

I. Het eerste deel bestaat uit een introductie tot het onderzoek en de onderzoeksmethodologie.

II. In het tweede deel wordt eerder onderzoek verzameld, samengevat en besproken met specialisten uit de praktijk om de relevantie en de eisen voor de volgende fase te bepalen.

III. In het derde deel worden de resultaten van deel II gebruikt om een volwassenheidsmodel bestaande uit focusgebieden en een beoordelingsinstrument te ontwerpen.

IV. Deel IV beschrijft drie cycli waarin opeenvolgde versies van het beoordelingsinstrument wordt gebruikt, geëvalueerd, gevalideerd en verbetert in de praktijk en een systematische literatuur studie naar de relatie tussen corporate governance en ITG.

V. Het laatste deel (deel V) bestaat uit de conclusies en antwoorden op de onderzoeksvragen, beperkingen en vervolgonderzoek.

De onderzoeksmethodologie is gebaseerd op ontwerp wetenschap (design science) en een combinatie van systematische literatuur studies, Delphi workshops en praktijk casussen. Een gedetailleerd overzicht van de structuur van deze dissertatie is opgenomen in sectie 2.4 en in het overzicht van het onderzoek in figuur 6.

In een Delphi workshop met een groep van CIO's is de relevantie van het onderzoek voor de praktijk gedemonstreerd en een systematische literatuurstudie naar ITG is verricht om bestaande kennis te verzamelen.

Het onderzoek dat gepresenteerd is in deze dissertatie heeft een multidisciplinair karakter. Het fundament ligt in onderzoek naar informatiesystemen, maar er zijn sterke banden met organisatie- en sociale wetenschappen. Organisaties kunnen gedefinieerd worden als sociale eenheden van mensen die gestructureerd en geleid worden om gemeenschappelijke doelen na te streven.

ITG kan dus vanuit twee perspectieven worden bekeken:

- $\quad$ een organisatorisch perspectief waar naar gerefereerd wordt als harde governance;

- $\quad$ een sociaal perspectief waar naar gerefereerd wordt als zachte governance.

Dit onderzoek is gebaseerd op de aanname dat voor de verbetering van de volwassenheid van organisaties aandacht moet worden gegeven aan zowel de harde als de zachte kant van ITG. De harde kant is gerelateerd aan processen en structuren, de zachte kant aan sociale aspecten zoals gedrag en organisatiecultuur. 
De stelling was dat onvolwassen of lage niveaus van ITG volwassenheid de oorzaak zijn voor het ontbreken van effectieve ITG. Om de ITG te verbeteren is gekozen een volwassenheidsmodel voor harde en zachte ITG te ontwerpen omdat een dergelijk model niet bestaat en nodig is in de praktijk. Dit volwassenheidsmodel is bedoeld om organisaties te helpen met het verbeteren van de harde (structuur, processen) en zachte (gedrag en samenwerking) ITG.

In Delphi workshops met ITG experts vanuit de praktijk zijn de focusgebieden voor een ITG volwassenheidsmodel voor harde en zachte governance bepaald. Tijdens de workshops is gebruik gemaakt van het groepsbesluitvormingsysteem Spilter.

Het eindresultaat (MIG model versie 1) is een volwassenheidsmodel dat bestaat uit drie delen: zachte governance, harde governance en de context (van een organisatie). Het volwassenheidsdeel van het MIG model bestaat uit harde en zachte governance. De context is buiten het volwassenheidsdeel geplaatst. Het MIG model bevat vier focusgebieden voor zachte governance: continue verbetering, leiderschap, participatie en onderling begrip en vertrouwen. Er zijn vijf focusgebieden voor harde governance: functies en rollen, besturingsorganen, IT besluitvorming, planning en monitoring. Er zijn drie focusgebieden in de context: cultuur, informele organisatie en sector. Het model is het MIG model genoemd.

Het MIG model is gebruikt voor het ontwerp van een ITG volwassenheidsmodel. Het artefact dat resulteerde uit het proces is het MIG assessment instrument genoemd. Deze dissertatie bevat een gedetailleerde beschrijving van het ontwerpproces in de periode van 2014 tot 2017. De beschrijving start met het ontwerpproces van de initiële versie, het protocol voor het gebruik van het instrument en de wijzigingen in de tweede en derde versie van het instrument. Het ontwerp van de eerste versie was gebaseerd op het MIG model en de resultaten van een serie Delphi workshops met specialisten uit de praktijk.

Het beoordelingsinstrument is gebruikt in praktijkcasussen die zijn uitgevoerd door studenten en door de onderzoekers. De wijzigingen in de tweede en derde versie van het MIG assessment instrument zijn gebaseerd op het gebruik, de verbetering en validatie van het instrument in diverse praktijkcasussen. De aanname is dat als studenten het instrument kunnen gebruiken dan kan verwacht worden dat specialisten uit de praktijk - die over het algemeen veel meer praktijkervaring hebben - zeker in staat zijn het instrument te gebruiken.

Tussen 2015 en 2017 zijn achtentwintig praktijkcasussen uitgevoerd waarbij gebruik is gemaakt van de eerste, de tweede en de derde versie van het instrument. Het gebruik van het instrument is altijd gecombineerd met semigestructureerde interviews om samen met de deelnemers de resultaten te evalueren en te corrigeren. Het succes van een volwassenheidsmodel is bewezen "als het resulteert in een discussie over verbeteringen onder de doelgroep" (Lasrado 
et al., 2017). De deelnemers reageerde positief op de bruikbaarheid van het instrument in het algemeen zoals "Het levert heel snel een indicatieve indruk van de volwassenheid van diverse ITG processen." of "De beoordeling en de interviews stimuleren mij. Daarmee heeft het al waarde voor mij." maar ook een aanzienlijke lijst met voorgestelde verbeteringen voor het instrument.

Omdat er vijf jaar zijn verstreken sinds de initiële literatuurstudie is uitgevoerd is deze in 2017 / 2018 herhaald. De update toonde dat het nog steeds hoogstwaarschijnlijk een discrepantie is tussen de ITG praktijk, de raamwerken (frameworks) en de literatuur. Daarnaast zijn vijf relatief nieuwe volwassenheidsmodellen voor ITG gevonden (inclusief het model dat onderwerp is van deze dissertatie). Slechts twee raamwerken bleken de harde en zachte ITG te omvatten: COBIT 5.0 op een holistische manier en het MIG model op een meer praktische manier.

De evaluaties toonden aan dat de combinatie van het instrument met semigestructureerde interviews, resulteert in een geavanceerd en bruikbaar instrument om het huidige niveau van de harde en zachte ITG van een organisatie te bepalen. Het onderzoek in deze dissertatie en het MIG assessment instrument demonstreert een manier om de discrepantie tussen ITG volwassenheidsliteratuur en praktijk te reduceren.

Corporate governance en ITG zijn gerelateerd en ITG kan gezien worden als een element van corporate governance. Er is weinig bekend over de manier waarop deze relatie werkt. Een systematische literatuurstudie naar corporate governance in 2018 is uitgevoerd om te valideren dat de twaalf focusgebieden die in het MIG model gebruikt worden voorkomen in hedendaagse corporate governance literatuur. Dit sluit aan op de ITG stroming dat ITG definieert als een deel van corporate governance. De literatuur review demonstreerde dat alle twaalf focusgebieden van het MIG model ook door corporate governance worden afgedekt.

Dit bevestigd dat de focusgebieden van het MIG model ook relevant zijn voor corporate governance. En dus, relevant voor ITG en corporate governance. Het laat verder zien dat de informele organisatie ook een contextuele factor van corporate governance is.

De bijdrage van het onderzoek dat beschreven is in deze dissertatie is tweeledig:

\section{Wetenschappelijke bijdrage}

Deze dissertatie illustreert dat voor organisaties de zachte kant van ITG minstens zo belangrijk is. Dit gaat (veel) verder dan relationele mechanismen die voorkomen in hedendaagse literatuur.

Ondanks alle inspanningen blijft het causale verband tussen ITG en de performance van een organisatie onduidelijk evenals de ITG mechanismen die invloed hebben op organisatorische performance. Het MIG model en het MIG assessment instrument kunnen dienen als een 
bruikbaar model om verder onderzoek mee uit te voeren om een beter begrip te krijgen van deze relaties.

De focusgebieden van het MIG model zijn ook relevant voor corporate governance. Het model en het instrument zijn dus mogelijk ook bruikbaar om een beter begrip te krijgen van hoe de relatie tussen ITG en corporate governance werkt.

\section{Bijdrage aan de praktijk}

Consultants in de praktijk baseren hun aanpak op basis van beschikbare modellen, raamwerken en ervaring. Het MIG model is een model dat ontwikkelt is met behulp van ontwerp wetenschap in samenwerking met specialisten uit de praktijk. Het onderzoek illustreert dat CIO's graag de beschikking hebben over een betrouwbaar meetinstrument.

Het MIG instrument is een praktische implementatie van het MIG model en kan daarmee dienen als een bruikbaar hulpmiddel om medewerkers en consultants te helpen de ITG in hedendaagse organisaties te verbeteren. 



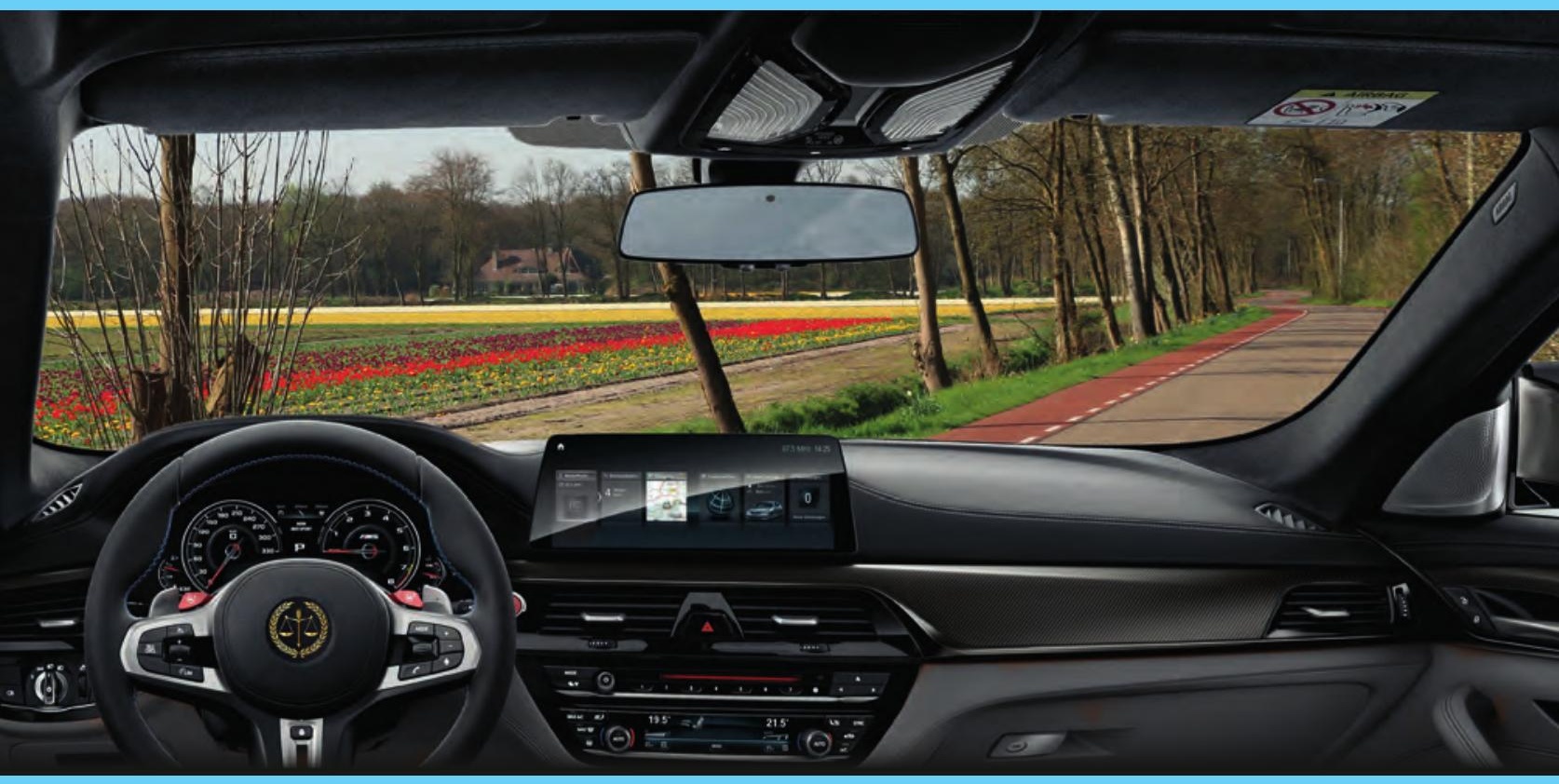

UNIVERSITY OF TWENTE.

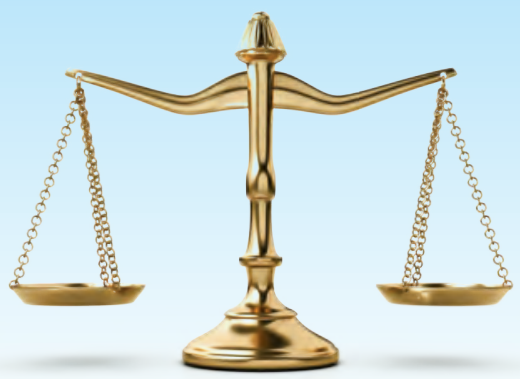

(C) Daniel Smits 